\title{
Characterization of the multifunctional XPG protein during Nucleotide-excision-repair
}

\author{
Doctoral Thesis \\ In partial fulfillment of the requirements for the degree \\ "Doctor rerum naturalium (Dr. rer. nat.)" \\ in the Molecular Medicine Study Program \\ at the Georg-August University Göttingen
}

submitted by

\section{Steffen Schubert}

born in Leinefelde, Germany

Göttingen, 31.01.2014 


\section{Members of the thesis committee}

\section{Supervisor:}

Prof. Dr. Steffen Emmert

University Medical Center, Dept. of Dermatology, Venereology and Allergology

Georg-August University Göttingen

First member of the thesis committee:

Prof. Dr. Michael P. Schön

University Medical Center, Dept. of Dermatology, Venereology and Allergology

Georg-August University Göttingen

Second member of the thesis committee:

Prof. Dr. Jürgen Brockmöller

University Medical Center, Dept. of Clinical Pharmacology

Georg-August University Göttingen

Third member of the thesis committee:

PD Dr. Wilfried Kramer

Institute for Microbiology and Genetics, Dept. of Molecular Genetics

Georg-August University Göttingen

Date of Disputation: 
AFFIDAVIT

Here I declare that my doctoral thesis entitled

"Characterization of the multifunctional XPG protein during Nucleotide-excision-repair" has been written independently with no other sources and aids than quoted. 
I would like to thank my supervisor Prof. Steffen Emmert for giving me the opportunity to write the thesis in his lab and for all the successful publications during this time. Special thanks go to the DFG graduate school 1034 (GRK1034) for providing a scholarship, several travel grants and an altogether remarkable financial support within the last years. Furthermore, I would like to thank Andreas Ohlenbusch and colleagues for the chance to use the capillary sequencer of the pediatrics department and for loading my probes uncountable times. I also want to thank Ron Gary (UNLV, USA) for providing the FF and Del plasmids and Dr. Toshio Mori (Nara Medical University, Japan) for providing the CPD antibodies. Moreover, I am obliged to all my lab mates, Christina Seebode, Stina Schiller, Regina Tiede, Petra Laspe, and Annika Schäfer for their manifold contribution and encouragement during the 3.5 years. Especially, I want to thank Antje Apel und Janin Lehmann, for the great and continuous support during every day work and during the last 3-4 months in particular, when everything piled up. Only with their help I was able to complete this thesis in time and largely compensate the first 1.5 years of my phD candidacy which I wasted with another project. I would like to thank all my friends and colleagues in Göttingen, at the first floor dermatology lab district, in the MolMed program, in the GRK1034, and in the Department of clinical pharmacology, for the backing and great moments in- and outside the lab. Last but not least, I want to thank my beloved wife Nelly for the extraordinary support during the whole time. She always kept my back, especially within the last 3-4 months, when I was constantly switching backwards and forwards between bench and desk, or did not leave the office at all. She helped me a lot to cure the "Denglisch" in this thesis, she took care of our son Karl Kito and placed herself and especially her own phD thesis back during this period, in order to create additional time for my work.

THANK you very, very MUCH! 


\section{List of Publications}

(1) Kuschal C., Thoms KM., Schubert S., Schäfer A., Boeckmann L., Schön MP., Emmert S.; Skin cancer in organ transplant recipients: Effects of immunosuppressive medications on DNA repair. Experimental Dermatology, 2012; 21, 2 - 6.

(2) Schäfer A.* , Emmert S.*, Kruppa J., Schubert S., Tzvetkov M., Mössner R., Reich K., Berking C., Volkenandt M., Pföhler C., Schön MP., Vogt T., König IR., Reichrath J.; No association of vitamin $\mathrm{D}$ metabolism-related polymorphisms and melanoma risk as well as melanoma prognosis: A case-control study. Archives of Dermatological Research, 2012; 304, 353 - 361.

(3) Schäfer A., Hofmann L., Gratchev A., Laspe P., Schubert S., Schürer A., Ohlenbusch A., Tzvetkov M., Hallermann C., Reichrath J., Schön MP., Emmert S.; Functional moleculargenetic analysis of 16 XP-C patients from Germany: Environmental factors predominately contribute to phenotype variations. Experimental Dermatology, 2013; 22, 24 - 29.

(4) Schäfer A., Schubert S., Gratchev A., Seebode C., Apel A., Laspe P., Hofmann L., Ohlenbusch A., Mori T., Kobayashi N., Schürer A., Schön M.P., Emmert S.; Characterization of 3 XPGdefective patients identifies 3 missense mutations that impair repair and transcription. Journal of Investigative Dermatology, 2013; 133, 1841 - 1849.

(5) Schäfer A., Gratchev A., Seebode C., Hofmann L., Schubert S., Laspe P., Apel A., Ohlenbusch A., Tzvetkov M., Weishaupt C., Oji V., Schön MP., Emmert S.; Functional and molecular genetic analyses of 9 newly identified XPD-deficient patients reveal a novel mutation resulting in TTD as well as in XP/CS complex phenotypes. Experimental Dermatology, 2013; $22,486-489$.

(6) Schubert S., Lehmann J., Kalfon L., Slor H., Falik-Zaccai T., Emmert S.; CUGC (clinical utility gene card) for Xeroderma Pigmentosum. Eur J Hum Genet, 2013

(7) Schubert, S.*, Lehmann, J.*, Schäfer, A., Apel, A., Laspe, P., Schiller, S., Ohlenbusch, A., Gratchev, A., Emmert, S.; An unusual mutation in the XPG gene leads to an internal in-frame deletion and a XP/CS complex phenotype. British Journal of Dermatology, 2014; under revision 


\section{Table of contents}

List of Publications III

List of Tables VIII

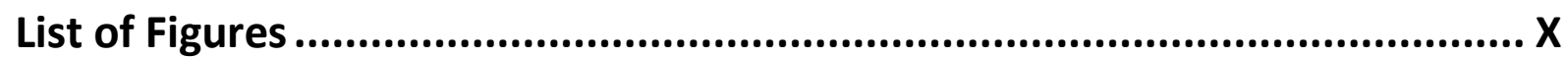

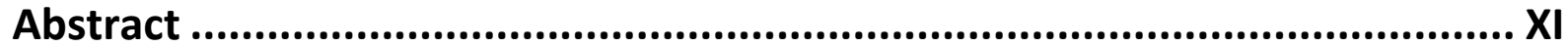

List of Abbreviations ................................................................................ XII

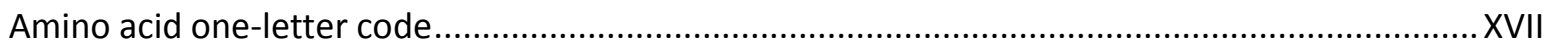

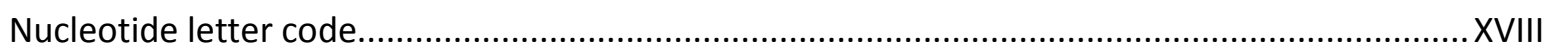

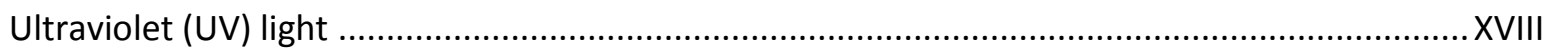

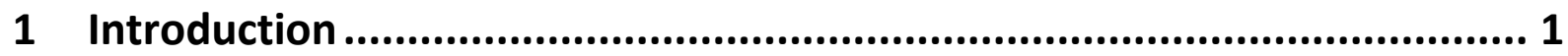

1.1 Multistep carcinogenesis model - malignant NER associated disorders - XP, CS and others 1

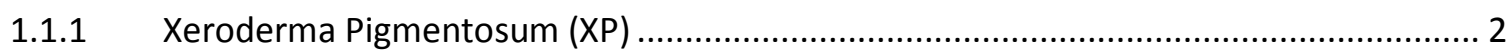

1.1.2 Cockayne Syndrome (CS) and combined XP/CS ................................................... 3

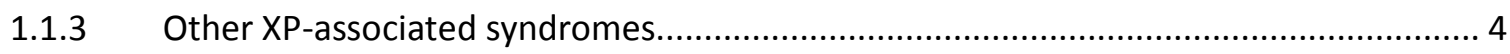

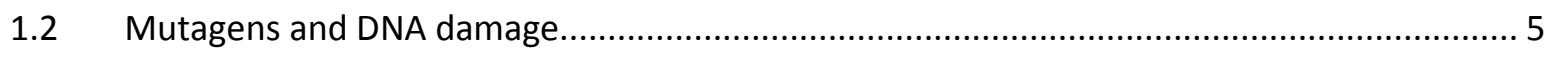

1.2.1 Endogenous DNA damage sources........................................................................... 5

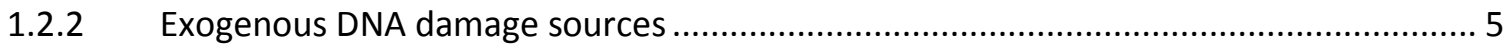

1.2.3 UV induced bulky DNA lesions in human DNA ..................................................... 5

1.3 Cells treat (UV) damage

1.3.1 The universal DNA damage response (DDR) ..................................................... 7

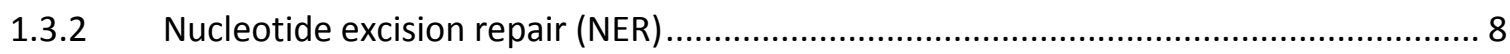

1.3.3 Damaged chromatin and Nucleotide excision repair ............................................. 11

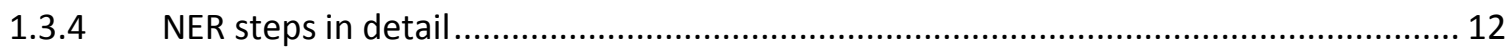

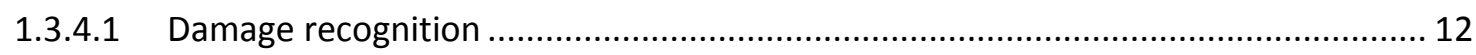

1.3.4.1.1 Damage monitoring during Global genome repair (GGR) ............................... 12

1.3.4.1.2 Damage recognition during Transcription coupled repair (TCR) ....................... 13

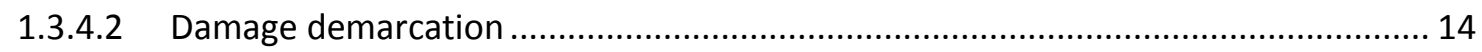

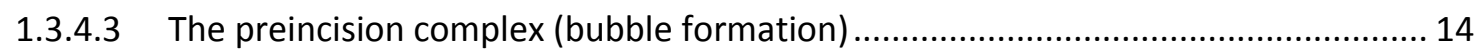


1.3.4.4 Dual incision complex and gap filling . 16

1.3.5 Proliferating Cell Nuclear Antigen (PCNA) acts during and after dual incision 19

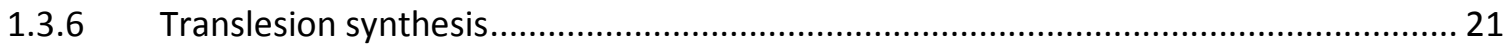

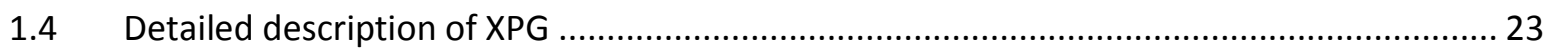

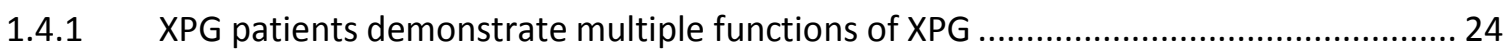

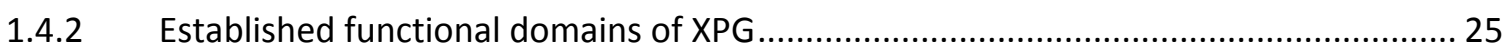

1.4.3 Structural and endonucleolytic function of XPG during NER and transcription ..........27

1.4.4 Involvement of XPG in other (DNA repair) pathways ........................................ 28

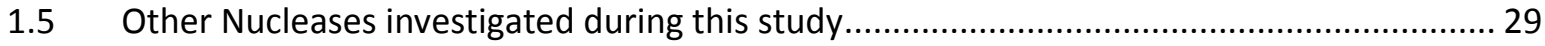

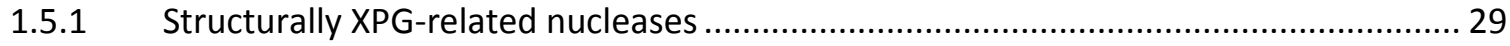

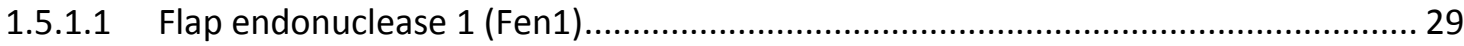

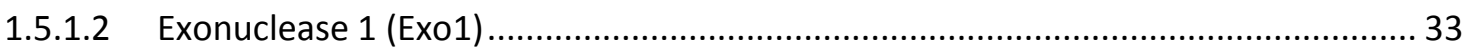

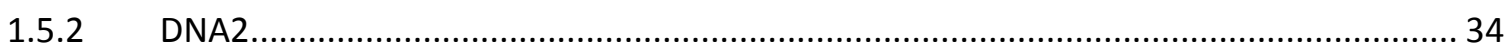

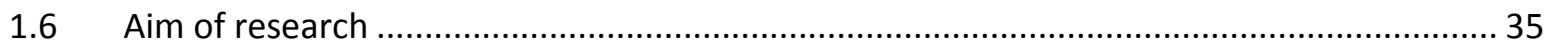

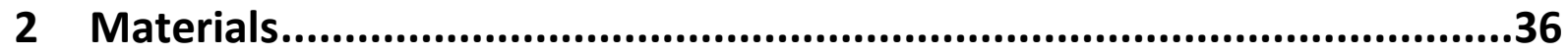

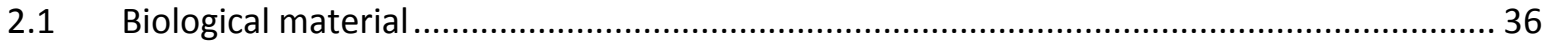

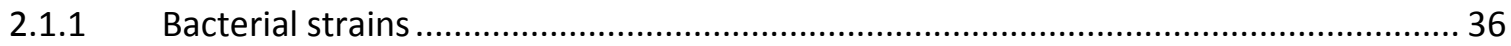

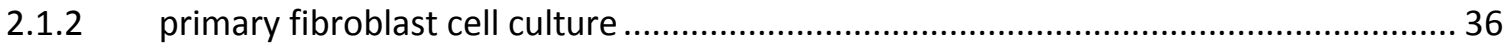

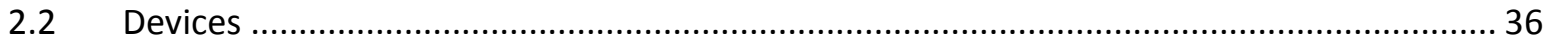

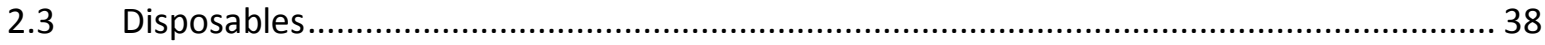

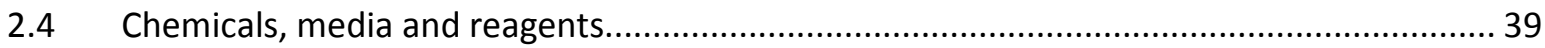

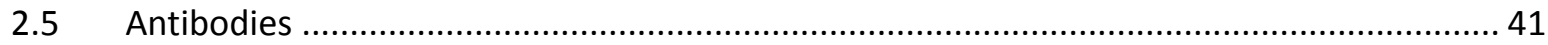

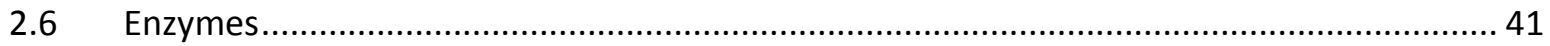

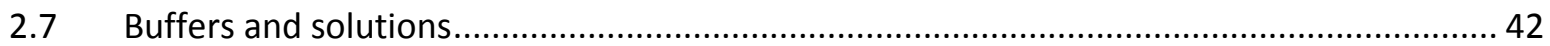

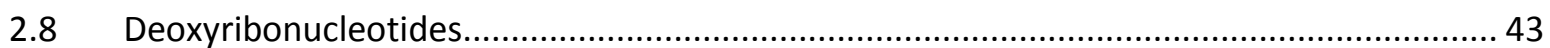

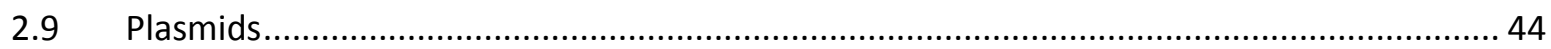

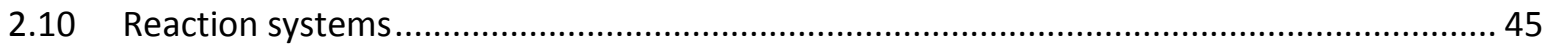

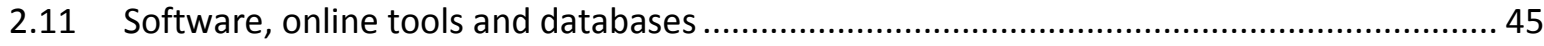

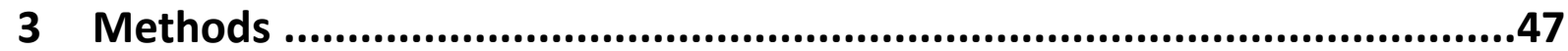

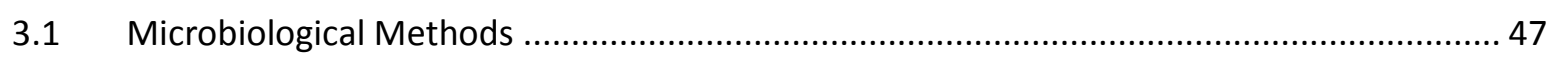

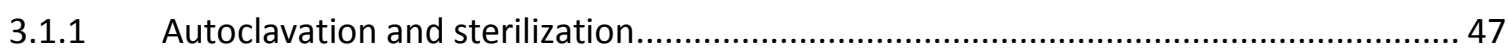




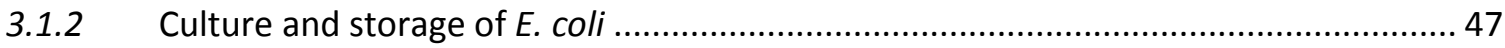

3.1.3 Photometric measurement of optical density of bacterial cultures ............................ 47

3.1.4 Preparation of chemically competent Escherichia coli ............................................... 47

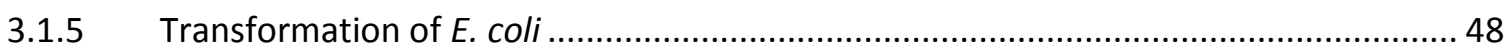

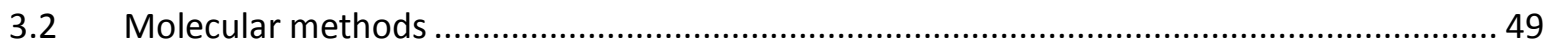

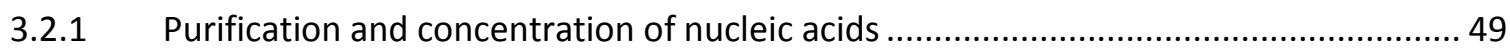

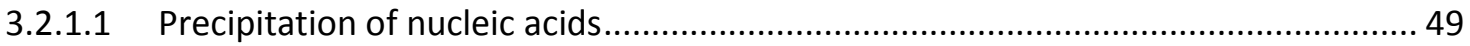

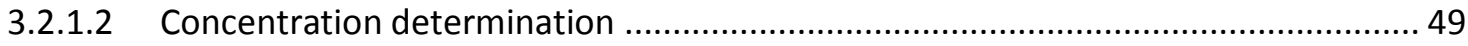

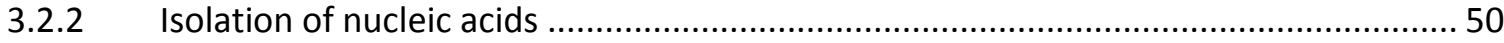

3.2.2.1 Extraction of nucleic acids from agarose gels ...................................................... 50

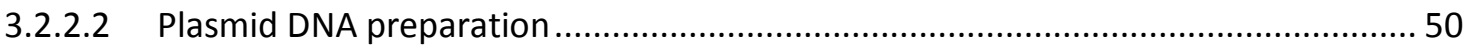

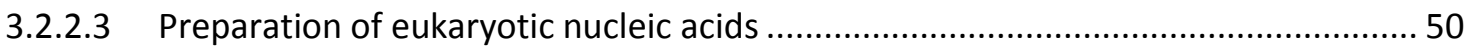

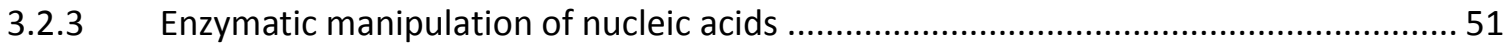

3.2.3.1 Complementary DNA (cDNA) synthesis .......................................................... 51

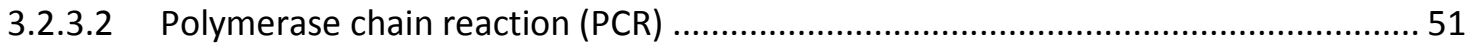

3.2.3.3 Site directed mutagenesis (Quick change) ....................................................... 52

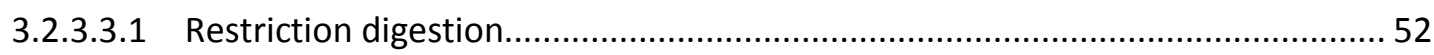

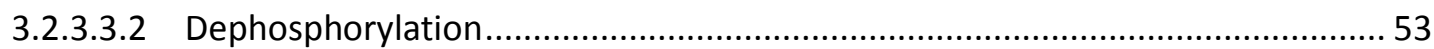

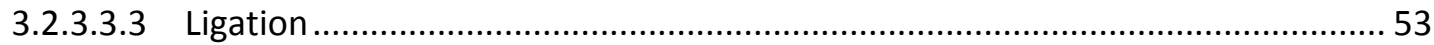

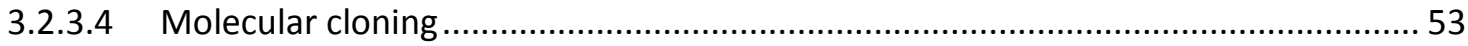

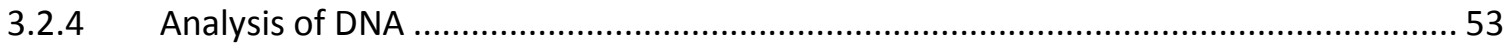

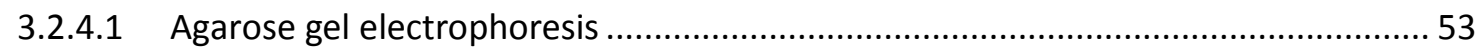

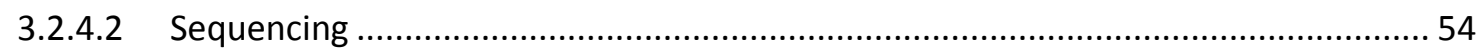

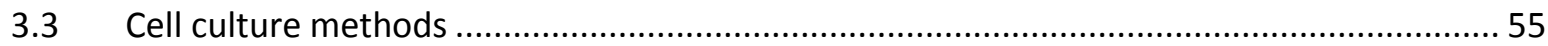

3.3.1 Culture of cells and preparation of primary cell cultures........................................... 55

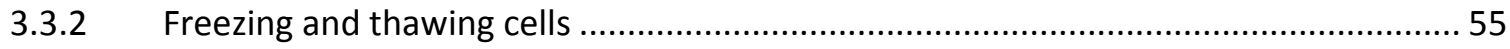

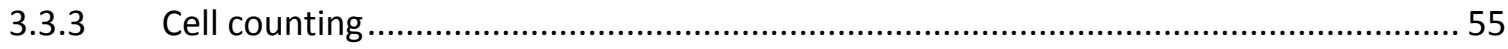

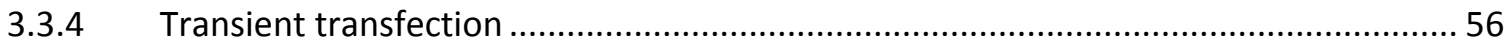

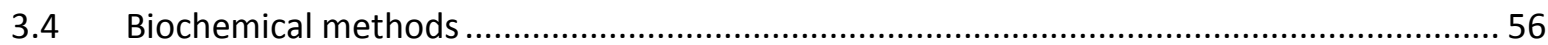


3.4.1 Preparation of whole cell protein lysates ............................................................... 56

3.4.2 Bradford protein quantification method (Bradford, 1976) .......................................... 57

3.4.3 Polyacrylamide gel electrophoresis (SDS-Page) (Laemmli, 1970) .............................. 57

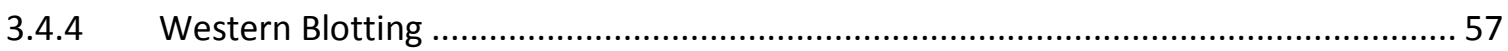

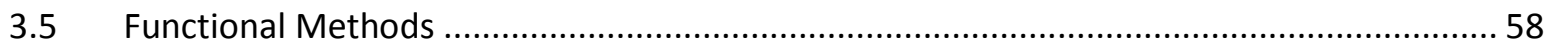

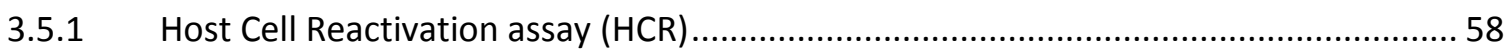

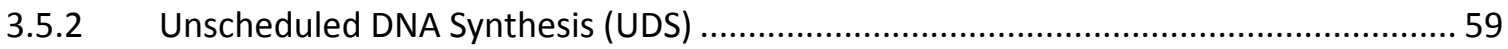

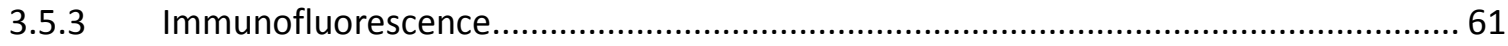

3.5.4 Triplestaining - simultaneous detection of DNA damage, DNA repair synthesis and

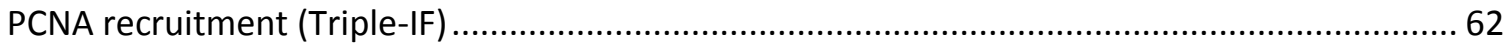

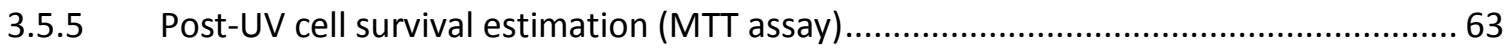

4 Results .64

4.1 Identification of a novel XPG patient and the corresponding mutant allele with

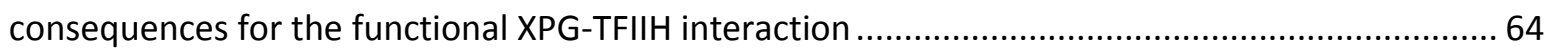

4.2 The functional interaction between PCNA and XPG is essential during NER ......................66

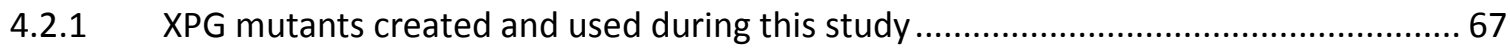

4.2.2 Immediate Unscheduled DNA repair synthesis (UDS) of XPG mutants .........................69

4.2.3 Host cell reactivation assessment of XPG mutants ................................................. 73

4.2.4 Short summary of the quantitative results .......................................................... 78

4.2.5 Estimation of simultaneous PCNA-recruitment and UDS synthesis at DNA damage sites

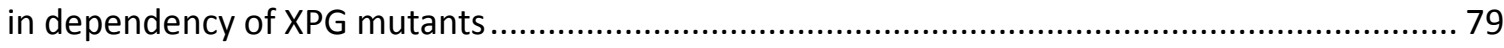

4.3 Investigations of other nucleases as a backup mechanism with regard to XPG Isoform VI . 80

5 Discussion

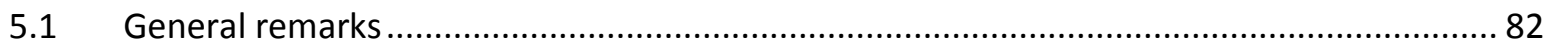

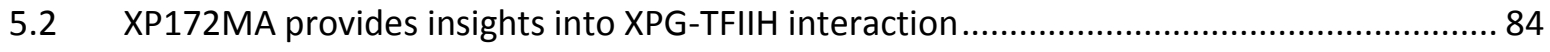

5.3 Genetic characterization of the functional XPG-PCNA interaction during NER ....................86 86

5.3.1 The C-terminal PIP-box of XPG is required for quick DNA repair synthesis but is unimportant for complementation of XPG 86

5.3.2 The functional N-terminal PCNA-XPG interaction is required for optimal NER ............ 89

5.4 Investigations on a backup mechanism for the XPG endonuclease function ....................... 92 
5.4.1 Endonuclease defective XPG (E791A) is able to complement XP20BE deficient cells .. 92

5.4.2 XPG Isoform VI supports complementation of XPG deficient cells and requires Fen1 endonuclease activity

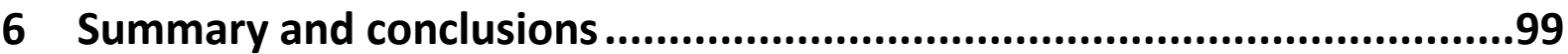

6.1 Model for the function of XPG during stalled NER intermediates ................................... 100

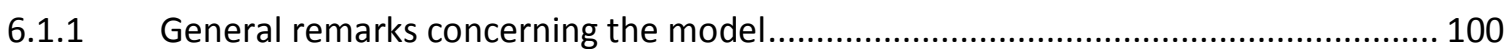

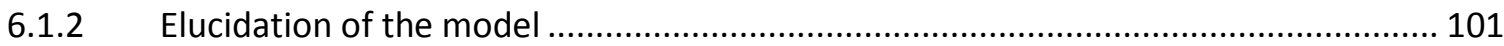

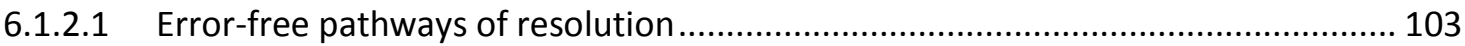

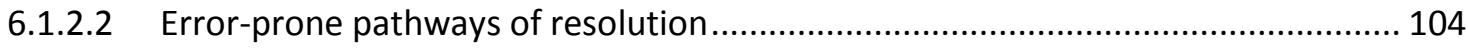

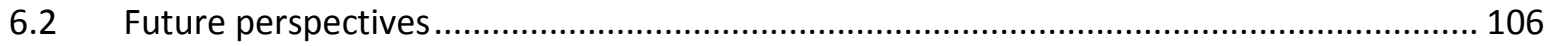

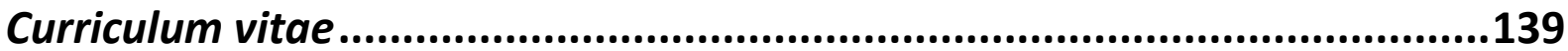

\section{List of Tables}

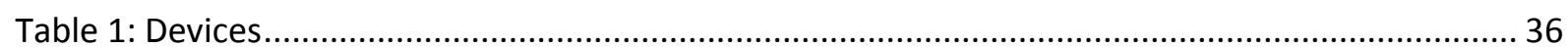

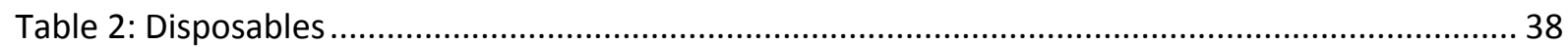

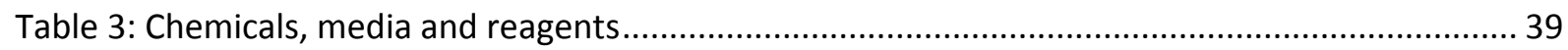

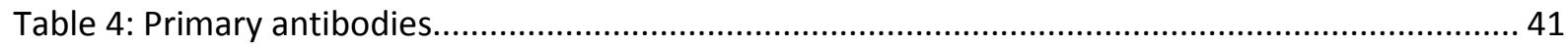

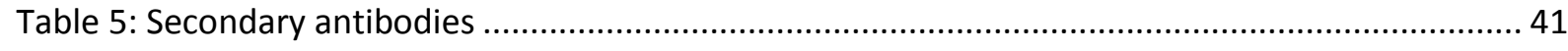

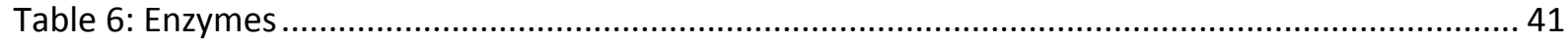

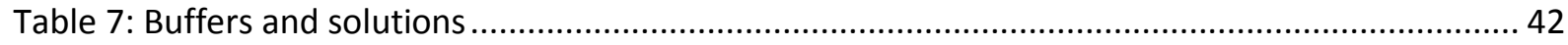

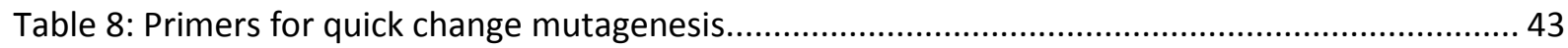

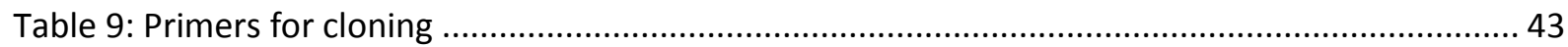

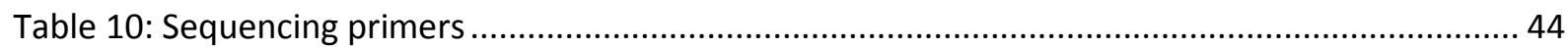

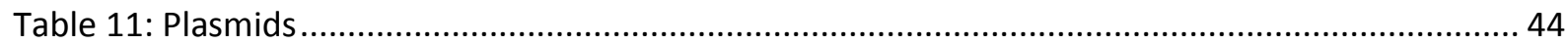

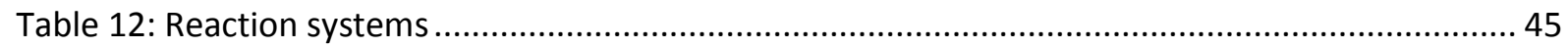

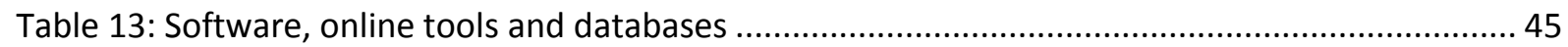

Table 14: Recombinant mutants of XPG and other enzymes used during this work...........................67

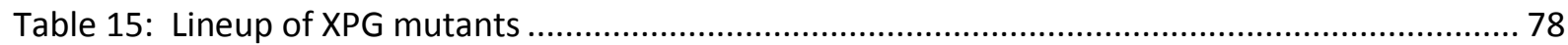


Table of contents 


\section{List of Figures}

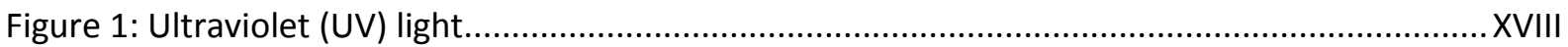

Figure 2: Overview of the Xeroderma Pigmentosum (XP) symptoms.................................................. 2

Figure 3: Genotype-phenotype relationship of XP-associated disorders …....................................... 4

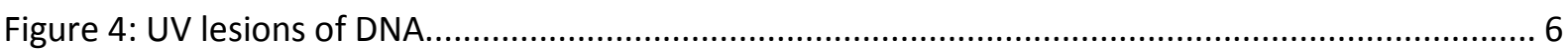

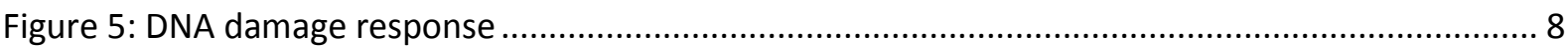

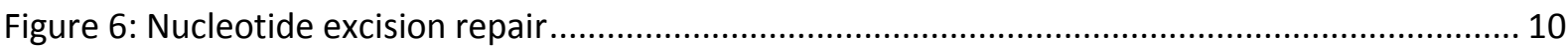

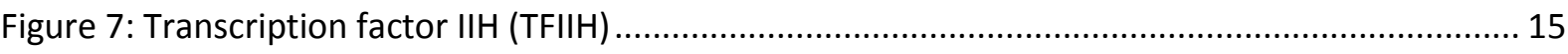

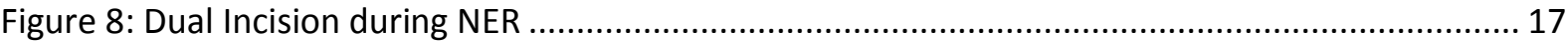

Figure 9: Ubiquitination independent Translesion synthesis (TLS) activity of Rev1 ........................... 23

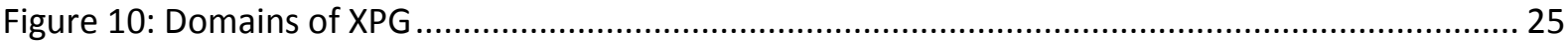

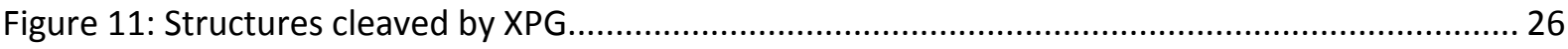

Figure 12: Fen1 superfamily domain conservation .......................................................................... 31

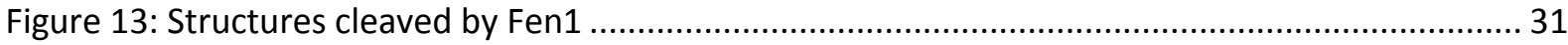

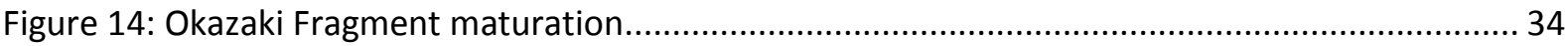

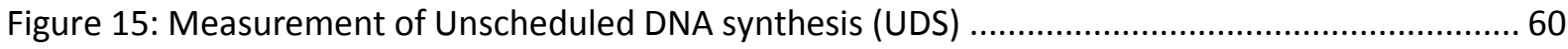

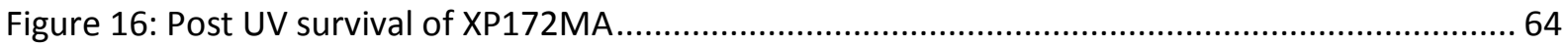

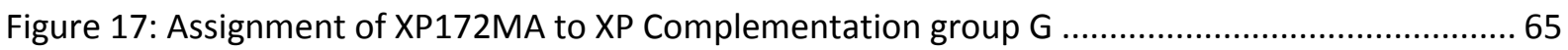

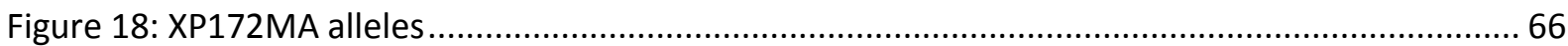

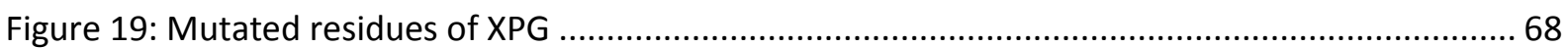

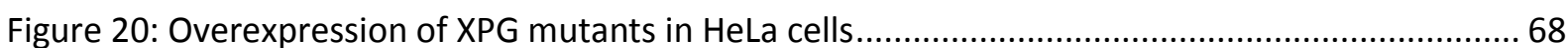

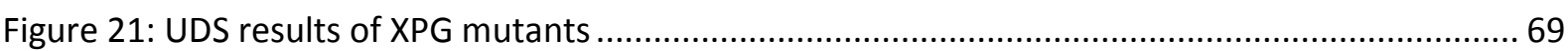

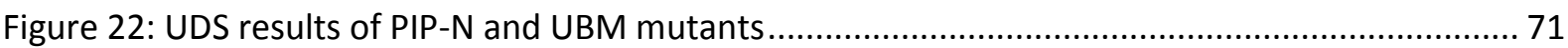

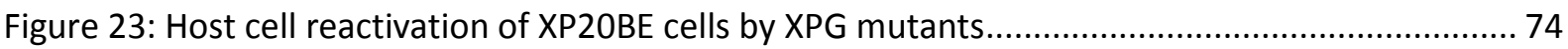

Figure 24: HCR of PIP- and UBM mutants as well as the respective double- and triple mutants......... 76

Figure 25: Triple staining (Triple-IF) of wt and XP20BE cells at different points in time after UV

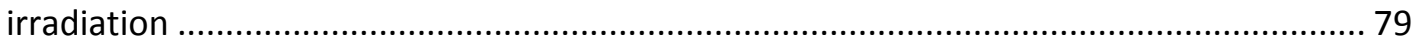

Figure 26: Assessment of UDS with respect to NER capability of Fen1 and DNA2 ............................. 81

Figure 27: Model for the function of XPG during stalled NER intermediates .................................... 100 


\section{Abstract}

Xeroderma pigmentosum (XP), a cancer model disease, is the perfect proof for the existing model of carcinogenesis activated by mutations. All patients share a defect in Nucleotide excision repair (NER). The gene, which is disease-causing for XP complementation group G (XPG) patients, encodes for the multifunctional endonuclease XPG. This enzyme has many binding partners like TFIIH, RPA and PCNA, and acts at a crucial step at the very end of NER. Several functional domains of XPG were mutated to investigate the behavior of the respective mutants during NER intermediates of dual incision, using DNA repair synthesis (UDS) and Host cell reactivation (HCR) assays. Furthermore, a new XPG patient with implications for the functional XPG-TFIIH interaction has been studied.

By genotype-phenotype correlation of a XPG patient (XP172MA), this study greatly suggests to narrow down the functionally important XPG interaction domain between TFIIH and XPG to the XPG amino-acids 30-85.

This study demonstrates that the functional PCNA-XPG interaction is more important for NER than the endonuclease function of XPG. The C-terminally located PIP-box of XPG is required for immediate UV response but not for the functionality of XPG during NER in transiently transfected primary fibroblasts. The N-terminal PIP-UBM ubiquitin binding domain is more important for integrity of NER than the C-terminal PIP-box.

I raise the model of an NER intermediate state that involves obligatory ubiquitination during NER and the blocking of error-prone translesion polymerases by XPG. This study excludes XPG as the responsible factor for PCNA recruitment and designates XPG as the factor as restrictive element for UV-damage dependent activation of translesion polymerases to S-phase.

The results obtained with the endonuclease defective E791A XPG mutant confirm the actual "cutpatch-cat-patch" model of dual incision during NER. Moreover, this study clearly demonstrates the ability of endonuclease defective XPG to perform accurate NER in living cells. This accounts for the existence of a cellular backup mechanism for the XPG endonuclease function.

The proposal for a nuclear backup mechanism is supported by the investigation of a physiologically relevant (evolutionary developed) XPG splicevariant with NER activity (IsoVI). The severely truncated XPG isoform is able to structurally complement a XPG defect. This complementation is dependent on the endonuclease function of Fen1. This suggests the existence of an evolutionary developed backup mechanism for XPG during NER. 


\section{List of Abbreviations}

\begin{tabular}{|c|c|}
\hline${ }^{\circ} \mathrm{C}$ & degree celsius \\
\hline$\mu g$ & micro gram \\
\hline 6-4PP & 6,4 pyrimidine-pyrimidones \\
\hline 8-oxo-G & 8-hydroxyguanine \\
\hline aa & amino acid(s) \\
\hline AP site & apurinic site \\
\hline APS & ammonium persulfate \\
\hline Arp & actin related proteins \\
\hline ATM & PI3K-like kinases ataxia-telangiectasia mutated \\
\hline ATP & adenosine triphosphate \\
\hline ATR & ataxia-telangiectasia and Rad3-related \\
\hline BAF/PBAF & polybromo- and BAF containing complex \\
\hline $\mathrm{BCC}$ & basal cell carcinoma \\
\hline BER (Ip, SN) & base excision repair (long patch, single nucleotide) \\
\hline bidest & double distilled water \\
\hline BLM & Bloom syndrome, RecQ helicase-like \\
\hline bp & base pairs \\
\hline bp & base pairs \\
\hline BPDE & benzo[a]pyrene-diol-epoxide-deoxyribonucleic acid \\
\hline BRCA1 & breast cancer type 1 susceptibility protein \\
\hline BSA & bovine serum albumin \\
\hline $\mathrm{CaCl}_{2}$ & calcium chloride \\
\hline CAF-1 & chromatin assembly factor \\
\hline CAK & cyclin activated kinase complex (TFIIH) \\
\hline Cat & catalogue \\
\hline CBP & CREB binding protein \\
\hline Cdc27 & Cell division cycle 27 \\
\hline CDK7 & cyclin-dependent kinase 7 \\
\hline CDK7 & cyclin dependent kinase 7 \\
\hline cDNA & complementary DNA \\
\hline CETN2 & Centrin 2 \\
\hline $\mathrm{CHK} 1,2$ & checkpoint kinase 1,2 \\
\hline CIAP & calf Intestinal Alkaline Phosphatase \\
\hline Cisplatin & cis-diamminedichloroplatinum(II) \\
\hline CMV & cytomegalovirus \\
\hline CPD & cyclobutane pyrimidine dimers \\
\hline CS & Cockayne syndrome \\
\hline
\end{tabular}




\begin{tabular}{|c|c|}
\hline CSA, CSB & Cockayne syndrome A, B protein \\
\hline CTCF & СССТC binding factor \\
\hline CTD & c-terminal domain \\
\hline Cul4A & cullin4A -dependent ligase E3 \\
\hline Cycl H & Cyclin $\mathrm{H}$ \\
\hline DDB1,2 & DNA damage binding protein 1,2 \\
\hline DDB1,2 & DNA damage binding protein 1,2 \\
\hline DDR & DNA damage response \\
\hline DFG & Deutsche Forschungsgemeinschaft \\
\hline $\mathrm{dH}_{2} \mathrm{O}$ & destilled water \\
\hline DMEM & Dulbecco's modified Eagle's medium \\
\hline DMSO & Dimethyl sulfoxide \\
\hline DNA & deoxyribonucleic acid \\
\hline DNA2 & DNA Replication Helicase/Nuclease 2 \\
\hline dNTP & deoxynucleotide triphosphates \\
\hline $\mathrm{dRP}$ & 5'-deoxyribose phosphate \\
\hline DSB & double strand break \\
\hline dsDNA & double stranded DNA \\
\hline DTT & dithiotreitol \\
\hline E. coli & Escherichia coli \\
\hline e.g. & for example \\
\hline E2, E3 & ubiquitin-conjugating enzyme, ubiquitin ligase, respectively \\
\hline EDTA & ethylendiamine tetraacetic acid \\
\hline EdU & ethynyl deoxyuridine \\
\hline ERCC1 & excision repair cross-complementing group 1 (short) \\
\hline etc & etcetera \\
\hline EtOH & ethanol \\
\hline Exo1 & exonuclease 1 \\
\hline FBS & fetal bovine serum \\
\hline Fen1 (DNase IV) & flap endonuclease 1 \\
\hline FITC & fluoresceinisothiocyanate \\
\hline for & forward \\
\hline G1/2- phase & growth phase 1,2 \\
\hline GEN & gap endonuclease activity \\
\hline GER & Germany \\
\hline GFP & green fluorescence protein \\
\hline GGR & Global genome repair \\
\hline $\mathrm{H}$ & histone \\
\hline $\mathrm{H} 2 \mathrm{HT}$ & helix - 2turn - helix \\
\hline
\end{tabular}




\begin{tabular}{|c|c|}
\hline HAT & histone acetyl transferase \\
\hline $\mathrm{HCl}$ & hydrochloric acid \\
\hline HCR & Host cell reactivation \\
\hline Hepes & 4-(2-hydroxyethyl)-1-piperazineethanesulfonic acid \\
\hline $\mathrm{hr}(\mathrm{s})$ & hour(s) \\
\hline HR23B & human Rad23 homolog B \\
\hline HR23B & Human homolog of Rad23 B \\
\hline HRP & hairpin repeat pathway \\
\hline $\mathrm{ICL}$ & interstrand crosslink \\
\hline IF & immuno fluorescence \\
\hline incl & including/inclusive \\
\hline INO80 & Arp4, Arp5, Arp8, and actin (INO80 complex) \\
\hline $\mathrm{J}$ & joule \\
\hline $\mathrm{k}$ & kilo \\
\hline $\mathrm{kDa}$ & kilo Dalton \\
\hline $\mathrm{KHCO}_{3}$ & potassium bicarbonate \\
\hline $\mathrm{Ku}$ & Ku protein \\
\hline LB & lysogeny broth (Bertani, 1951) \\
\hline $\mathrm{LD}_{50,90}$ & lethal dose with 50 or $90 \%$ survival, respectively \\
\hline Lig & ligase \\
\hline $\mathrm{m}$ & meter \\
\hline $\mathrm{M}$ & molar \\
\hline M-phase & mitotic phase \\
\hline $\mathrm{m} / \mathrm{v}$ & mass/volume \\
\hline MAT1 (MNAT1) & CDK-activating kinase assembly factor \\
\hline $\mathrm{MCMT}$ & Human DNA-(cytosine-5) methyl transferase \\
\hline MCS & multiple cloning site \\
\hline Med17 & mediator subunit 17 \\
\hline mer & repeat unit \\
\hline $\mathrm{MgCl}_{2}$ & magnesium chloride \\
\hline $\min$ & minute(s) \\
\hline $\mathrm{mM}$ & milli molar \\
\hline $\mathrm{mm}$ & millimeters \\
\hline MMR & mismatch repair \\
\hline MNNG & 1-Methyl-3-nitro-1-nitrosoguanidine \\
\hline mRNA & messenger RNA \\
\hline Muts & mutator S (mismatch repair) \\
\hline $\mathrm{Na}_{2} \mathrm{HPO}_{4} \times 2 \mathrm{H}_{2} \mathrm{O}$ & sodium hydrogen phosphate \\
\hline $\mathrm{NaCl}$ & sodium chloride \\
\hline
\end{tabular}




\begin{tabular}{|c|c|}
\hline $\mathrm{NaHCO}_{3}$ & sodium bicarbonate \\
\hline $\mathrm{NaOH}$ & sodium hydroxide \\
\hline NER & Nucleotide excision repair \\
\hline $\mathrm{NH}_{4} \mathrm{OAc}$ & ammonium acetate \\
\hline NHEJ & non-homologues end joining \\
\hline NLS & nuclear localization signal \\
\hline $\mathrm{nm}$ & nano meter \\
\hline no & number \\
\hline NSCLC & non-small cell lung carcinoma \\
\hline $\mathrm{nt}(\mathrm{s})$ & nucleotide(s) \\
\hline NTH1 & endonuclease III homolog 1 \\
\hline $\mathrm{O}$ & Oxygen \\
\hline $\mathrm{o} / \mathrm{n}$ & over night \\
\hline$O D_{x x n m}$ & optical density at xx nm \\
\hline oligo & oligonucleotide \\
\hline $\mathrm{P} / \mathrm{S}$ & penicillin - streptavidin mix \\
\hline p21 & cyclin-dependent kinase inhibitor 1, CDK-interacting protein 1 \\
\hline P300 & EP300 or E1A binding protein p300 \\
\hline p34, p44, p52, p62 & TFIIH proteins with a certain molecular mass (kDa) \\
\hline p53 & protein $53 \mathrm{kDa}$, tumor suppressor (gene) \\
\hline p68 & protein 68 (Pol $\delta$ subunit) \\
\hline PAA & polyacrylamide \\
\hline PAGE & polyacrylamide gel electrophoresis \\
\hline PARP1 & Poly (ADP-ribose) polymerase 1 \\
\hline PBS & phosphate-buffered saline \\
\hline PCNA & proliferating cell nuclear antigen \\
\hline PCR & polymerase chain reaction \\
\hline PEG & polyethylene glycol \\
\hline PFA & paraformaldehyde \\
\hline $\mathrm{pH}$ & potentium hydrogenii \\
\hline Pif1 & 5'3' helicase Pif1 \\
\hline PIP & PCNA interacting peptide \\
\hline Pol & polymerase \\
\hline Rad & Rad proteins \\
\hline RBX1 & RING-box protein 1 \\
\hline $\operatorname{RecQ}$ & RecQ helicase \\
\hline rev & reverse \\
\hline Rev1 & DNA repair protein Rev1 (TLS Pol) \\
\hline RFC & replication factor $\mathrm{C}$ \\
\hline
\end{tabular}


RLT buffer

RLUs

RNA

RNase A

ROS

RPA

rpm

RT

S- phase

s. (pombe, cerevisiae)

SD

SDS

sec

SEM

Sf9

SIRT1

SNP

SSC

ssDNA

SWI/SNF

TBE

TBP

TCR

TEMED

TFIIH

TLS

Triple-IF

Tris

TTD

TTDA (p8)

U

ubi

UBM

UBZ

UDS

UMG

Ung2

USA

UTR buffer Qiagen

relative light units

ribonucleic acid

Ribonuclease A

reactive oxygen species

replication protein $\mathrm{A}$

rounds per minute

room temperature

synthesis phase

Schizosaccharomyces

standard deviation

sodium dodecyl sulfate

seconds

standard error of the mean

Spodoptera frugiperda

Sirtuin 1

single nucleotide polymorphism

squamous cell carcinomas

single stranded DNA

SWItch/Sucrose Non Fermentable

Tris/borate/EDTA

TATA binding protein

transcription coupled repair

$\mathrm{N}, \mathrm{N}, \mathrm{N}, \mathrm{N}$-tetramethyl-ethane-1,2-diamine

basal transcription factor IIH

translesion synthesis

triple-fluorescence staining

Tris(hydroxymethyl)-aminomethane

Trichothiodystrophy

trichothiodystrophy A

Unit

ubiquitin

ubiquitin-binding motif

ubiquitin-binding zinc finger

unscheduled DNA synthesis

University medical center Göttingen

Nuclear uracil-DNA glycosylase

United states of America

untranslated region 


$\begin{array}{ll}\text { UV }(A, B, C) & \text { Ultraviolet A,B,C } \\ \text { UVR-A,B,C } & \text { (A)BC exinuclease } \\ \text { UVR-D } & \text { DNA polymerase II } \\ V & \text { Volts } \\ \text { V/v } & \text { volume/volume } \\ \text { W/v } & \text { weight/volume } \\ \text { WRN } & \text { WS 3'-5' RecQ-like helicase } \\ \text { WS } & \text { Werner syndrome } \\ \text { wt } & \text { wild type } \\ \text { Xg } & \text { acceleration of gravity } \\ \text { XAB2 } & \text { XPA Binding Protein 2 } \\ \text { XP } & \text { Xeroderma Pigmentosum } \\ \text { XP(G)/CS } & \text { XP(G) and CS combined syndrome, distinct clinical entity } \\ \text { XP-A to G, V } & \text { XP complementation groups A to G, variant } \\ \text { XRCC1 } & \text { X-ray repair cross-complementing protein 1 } \\ \mu l & \text { micro liter } \\ \mu M & \text { micro molar }\end{array}$

\section{Amino acid one-letter code}

\begin{tabular}{cc} 
one-letter code & amino acid \\
\hline \hline A & Alanine \\
C & Cysteine \\
D & Aspartic acid \\
E & Glutamic acid \\
F & Phenylalanine \\
G & Gylcine \\
H & Histidine \\
I & Isoleucine \\
K & Lysine \\
L & Leucine \\
N & Asparaginine \\
P & Proline \\
Q & Glutamine \\
R & Arginine \\
S & Serine \\
T & Threonine \\
V & Valine \\
W & Trypthophane \\
Y & Tyrosine
\end{tabular}




\section{Nucleotide letter code}

\begin{tabular}{cc} 
one-letter code & base \\
\hline \hline A & Adenine \\
C & Cytosine \\
G & Guanine \\
T & Thymidine
\end{tabular}

\section{Ultraviolet (UV) light}

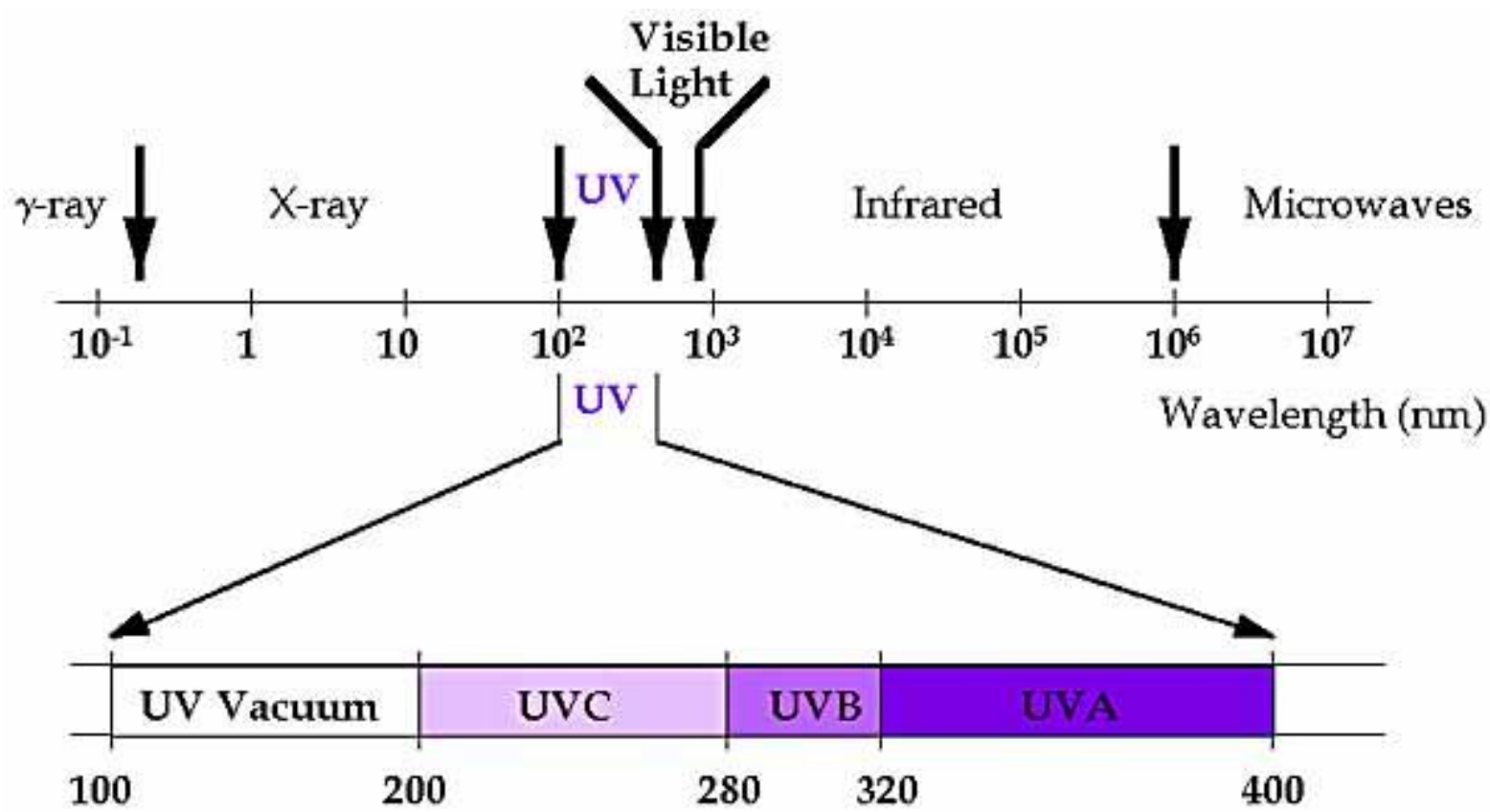

Figure 1: Ultraviolet (UV) light

UV light is divided into short wave UVC, middle wave UVB and long wave UVA light (Soehnge et al, 1997). Both UVA and UVB reach the earth's surface (the eyes, the skin) in sufficient amounts to provoke DNA damage (see chapter 1.2.2) in living species and in turn to be carcinogenic (see chapter 1.1). Normally, UVC is effectively blocked from reaching the earth's surface by the ozone layer of the atmosphere, although carries the highest energy and is used during the experiments to gain DNA damage. 
Introduction

\section{Introduction}

All living species are constantly exposed to endogenous and environmental deoxyribonucleic acid (DNA) damaging agents which jeopardize integrity of nucleic acids, mainly by base modifications (Lindahl, 1993). Among other things, unrepaired DNA damages in coding or functional chromosomal areas can lead to mutations (see chapters 1.2.3, 1.3.6) in the DNA sequence content after error prone replication. This in turn alters the amino acid sequence and functions of its gene products (Jena, 2012). Moreover, malfunctions of enzymes and proteins (mutated tumor suppressors or activated oncogenes) can drive the cellular transformation towards cancer (Hoeijmakers, 2009).

\subsection{Multistep carcinogenesis model - malignant NER associated disorders - XP, CS and others}

Human cancer is, among other things, hallmarked by the accumulation of errors (Hanahan \& Weinberg, 2000). Mutations in the genome are the first key step to the activation of oncogenes or the inhibition of tumor suppressor genes. This results in the loss of cell cycle control and apoptosis (Soehnge et al, 1997), thus in carcinogenesis.

In squamous cell carcinomas (SCCs) and basal cell carcinomas (BCCs) the typical UVB light (see Figure 1, Figure 2) derived CC to TT mutation pattern is found in the defective $p 53$ tumor suppressor gene (Dumaz et al, 1993; Ehrhart et al, 2003). P53 is a multiplayer in the cell and fulfills a lot of functions (it activates cell cycle checkpoints and DNA repair pathways and initiates apoptosis), has over 100 interaction partners and is seen as the major trigger of human cancer development in all tissues and is involved in the DNA damage response (Greenblatt et al, 2003) (see chapter 1.3.1). In contrast to other cancers, e.g. colon cancers, where p53 is affected at relatively late stages of the malign progression from adenoma to carcinoma (Fearon \& Vogelstein, 1990), in skin cancers p53 mutations arise at early onset stages (Campbell et al, 1993). The oncogenic transformation is further induced by expression level changes due to transcription factor binding inhibition especially by the above mentioned two adjacent cytosine to tyrosine transitions, effecting the TATA-box consensus sequence and thus binding of TATA binding protein (TBP) (Tommasi et al, 1996)

Xeroderma pigmentosum (XP), an interesting cancer model disease is the perfect proof for the existing model of carcinogenesis activated by mutations (Cleaver, 2000). Patients may suffer from mutations in different genes but share a common feature, a defect in Nucleotide excision repair (NER, see chapters 1.3.2, 1.3.4), this, in turn, results in the accumulation of mutations by action of the error prone translesion polymerases, e.g. polymerase (Pol) $\zeta$, during replication (Gan et al, 2008). 


\subsubsection{Xeroderma Pigmentosum (XP)}

Xeroderma pigmentosum (XP, greek xeros = dry, "dry pigmented skin") was first described by Hebra and Kaposi in 1874 (DiGiovanna \& Kraemer, 2012). Xeroderma pigmentosum is a rare autosomal recessive disorder, characterized among other symptoms by extreme photosensitivity of the skin and eyes, pigmentary changes (freckle-like skin), premature skin aging, and rapid malignant tumor development (Figure 2). A defect in the removal of UV-induced (bulky) DNA lesions, induced by ultraviolet (UV) light (see Figure 1), is characteristic for XP and many XP-related disorders, first described in 1968 (Cleaver, 1968). By complementation experiments (fusion of fibroblast from different XP patients) the heterogeneity of the disease was demonstrated, subsequently gene defects in the seven major NER-genes (XPA-XPG) were identified to be responsible for the clinical picture and became name-givers for the proteins in the NER pathway (see chapter 1.3.2) (Arase et al, 1979; Halley et al, 1979; Kraemer et al, 1975a; Kraemer et al, 1975b). Until today, XP patients and their cells in culture serve as the major model disease for NER research.
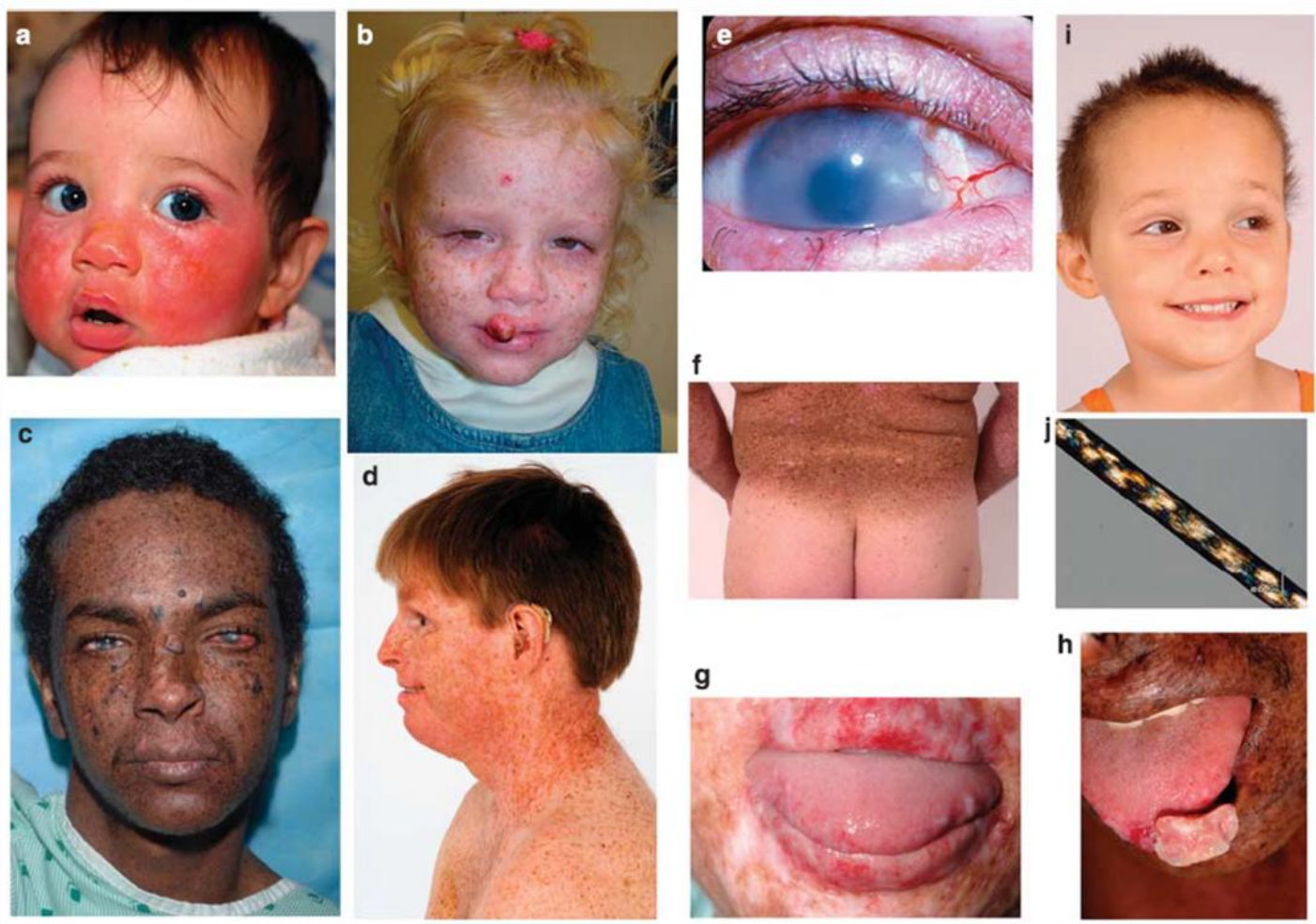

\section{Figure 2: Overview of the Xeroderma Pigmentosum (XP) symptoms}

A) XPD: severe blistering erythema of the malar area following minimal sun exposure; b) XPC: multiple hyper pigmented macules, a rapidly growing squamous cell carcinoma (SCC) or carcinoma on the upper lip and a precancerous lesion on the forehead; c) XPC: basal cell cancer on left nasal root and left cheek, the eyes show cornea scarring from unprotected sun exposure; d) XPA: numerous hyper pigmented macules on sun-exposed areas, additional neurological symptoms, sensorineural deafness; e) Corneal clouding, pterygium, contact lens, and loss of lashes on lower eyelid; f) Sharp demarcation of sun-exposed skin; g) Loss of vermillion border of the lips with prominent telangiectasias, scaring of the lips, and anterior tongue; h) SCC of the anterior tongue; $i, j$ ) short brittle hair and a smiling, outgoing personality typical for TTD-patients. (Bradford et al, 2011; DiGiovanna \& Kraemer, 2012; Liang et al, 2005; Mahindra et al, 2008) 
Xeroderma Pigmentosum has been diagnosed across all racial groups of all continents with its complementation groups XP-A to XP-G and XP variant (Schubert et al, 2013). The estimated prevalence of XP is 1: 1000000 in the USA and Europe and remarkably higher in Japan (1: 20000 ), especially due to XPA gene defects (Hirai et al, 2006; Kleijer et al, 2008). The incidence in Northern Europe, North Africa and the Middle East is assumed to be also elevated, particularly in cultures with an increased level of consanguinity or in long term isolated populations (Kraemer KH, 2003-2014). Noteworthy, only about $60 \%$ of affected individuals are characterized by an excessive and prolonged sunburn response (Bradford et al, 2011). The statistical numbers are differing between the sources, but the UV-dependent development of squamous cell carcinomas (SCC) or basal cell carcinomas (BCC) as well as melanoma skin cancers is extraordinary enhanced, 1000 to 10000 -fold and 1000 to 2 000-fold, respectively (Cohen \& Ellwein, 1991). Tumors are preferentially located at sun-exposed areas and arise in XP patients at the mean age of six to nine years and as the case may be 22 years for melanomas (Bradford et al, 2011; Cleaver, 2000; DiGiovanna \& Kraemer, 2012; Kraemer et al, 1987; van Steeg \& Kraemer, 1999). This is a great effect compared with normal e.g. the Caucasian population, wherein the first skin cancers are statistically developed about 50 years later (Bradford et al, 2011; Emmert \& Kraemer, 2013). Furthermore, the distribution of other cancers types is nearly identical between XP-patients and the normal population, accounting for the influence of the Nucleotide excision repair (NER) on cell constitution and transformation prevention (Kraemer, 1997; van Steeg \& Kraemer, 1999) (see chapter 1.3.2). Estimated over all complementation groups, the minority of XP cases shows additional neurological abnormalities, leading to phenotypes with varying severity (see chapters 1.1.2, 1.1.3, see Figure 2).

\subsubsection{Cockayne Syndrome (CS) and combined XP/CS}

The Cockayne Syndrome has an incidence of $1: 2.7$ million and patients exhibit XP symptoms like photosensitivity and freckling of sun-exposed skin, and furthermore cortically develop the characteristic bird-like facies with unusual deep set eyes, flat cheek bones, and neurological abnormalities like growth retardation (dwarfism), mental retardation, microcephaly, loss of ability to walk, deafness, and progressive visual loss because of pigmentary retinal degeneration (Dollfus et al, 2003; Kleijer et al, 2008; Nance \& Berry, 1992). CS arises mainly as a result of mutations in transcription coupled repair (TCR) - associated (see chapter 1.3.4.1.2) CSA and CSB genes (Kraemer et al, 2007). Interestingly, despite the fact that CSA and CSB are supposed to be involved in chromatin remodeling, CS patients do not exhibit an increased skin cancer risk like XP patients (Fousteri et al, 2006; Kraemer et al, 2007).

The combined phenotype, XP/CS-complex, arises mainly due to transcriptional missfunction and failures in the activation of nuclear receptors, because all three involved enzymes, XPB, XPD, and 
$\mathrm{XPG}$, are part of (XPB, XPD) or associated with (XPG) the universal transcription factor TFIIH (see chapters 1.3.4.31.3.4, 1.4) (Ito et al, 2007; Kraemer et al, 2007). XP/CS is phenotypically characterized by photosensitivity, an increased risk for the development of skin cancers and the typical CS symptoms like short stature, bird-like facies, mental retardation progressive neurological degeneration and deafness (Emmert et al, 2006; Kraemer et al, 2007).

\subsubsection{Other XP-associated syndromes}

DNA repair disorders

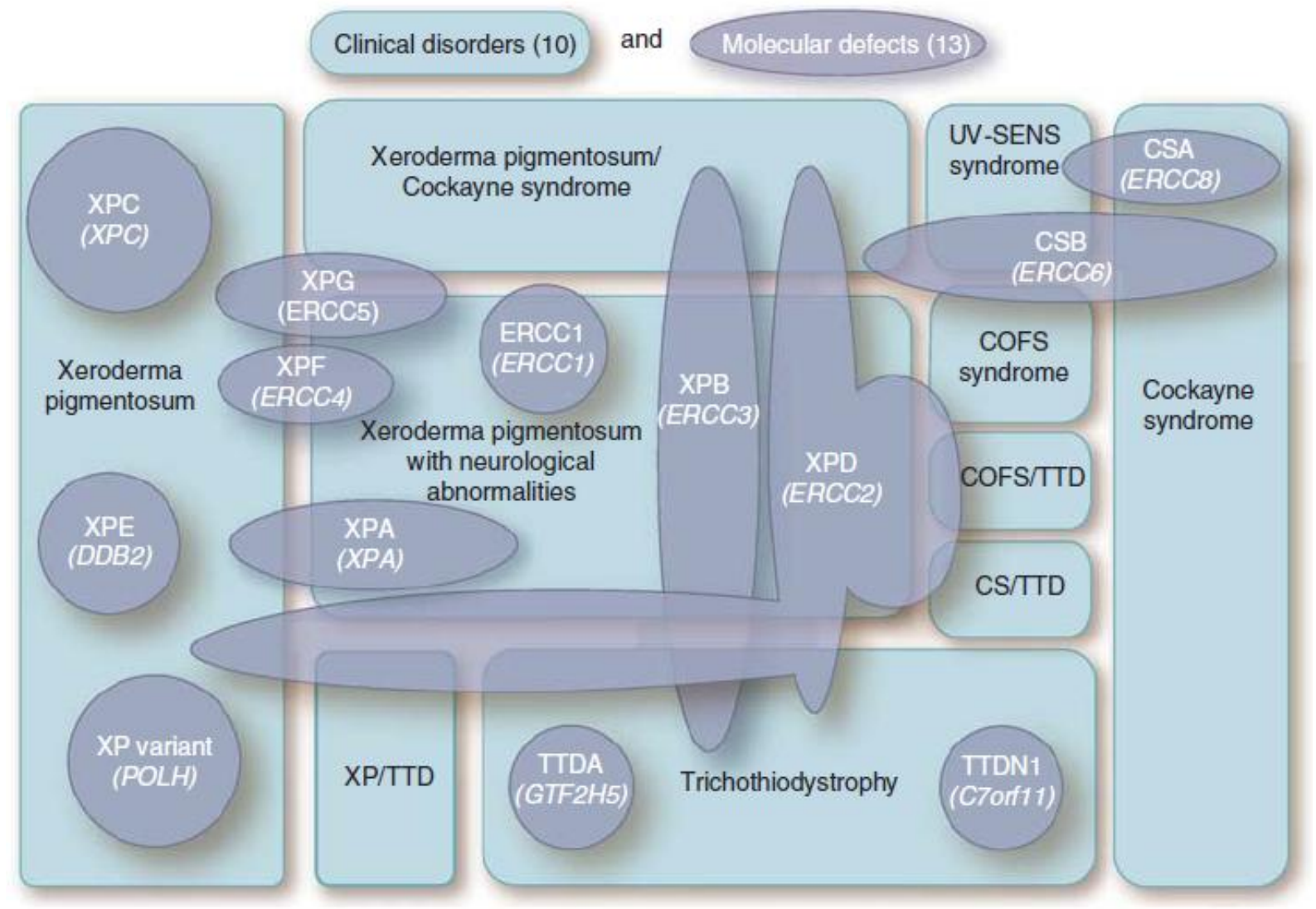

Figure 3: Genotype-phenotype relationship of XP-associated disorders

The violet circles depict the genotypes; the turquoise rectangles represent the several (combined) phenotypes. Different gene failures are able to cause manifold phenotypes and vice versa (DiGiovanna \& Kraemer, 2012).

There is a complex and yet not fully understood genotype-phenotype relationship between the different clinical entities of the XP-spectrum (see (DiGiovanna \& Kraemer, 2012; Le May et al, 2010). Beside Xeroderma pigmentosum, the Cockayne Syndrome (CS) and Trichothiodystrophy (TTD) are the major NER-deficient disorders and exist also in a phenotypical combination with $\mathrm{XP}$, depending on the location of the gene defect.

Figure 3 depicts a detailed scheme. For example mutations in XPG, XPD and XPB separately provoke the combined XP-CS phenotypes, in case of the latter two also in combination with TTD. 


\subsection{Mutagens and DNA damage}

The amount of spontaneous mutations due to replication events is not sufficient to trigger the cancer development. This chapter gives a brief overview of the sources of additional DNA damage.

\subsubsection{Endogenous DNA damage sources}

Several sources of DNA damage were identified including essential substances for life like water or oxygen (Tice, 1985). Reactions with water by far cause the most common intercellular types of DNA damages. They are comprising strand breaks (hydrolysis), depurination, depyrimidination, and deamination, which lead to abasic sites or base modifications like formation of uracil (deamination of cytosine; $\mathrm{G}$ to $\mathrm{A}$ transition) or Hypoxanthine (deamination of adenine; $\mathrm{T}$ to $\mathrm{C}$ transition) (Contreras \& Madariaga, 2003). Consequence is the formation of miss pairing base pairs and later on the establishment of wrong sequence context in daughter chromatids (transversions and transitions). Reactive oxygen species (ROS), formed by irradiation or chemical species generating free radicals like $\mathrm{O}_{2}^{-}$, add methyl or ethyl groups to nucleotides or oxygenate bases (e.g. Guanine), respectively (Prakash et al, 2000). Two of the most common types of base modifications in this context are $0^{6}$ methylguanine or 8-oxo-G which lead to $C$ to $T$ transitions. Some alkylating agents, e.g. nitrogen mustard, used for cancer treatment, are able to produce interstrand crosslinks (ICLs) like cisplatin, (McHugh et al, 2001). Alkylating agents (like MNNG) because the same type of mutations and arise from exogenous sources (see chapter 1.2.2).

\subsubsection{Exogenous DNA damage sources}

Another source of DNA damage is radiation. Ionizing radiation, $\alpha-, \gamma$ - or X-Rays, create double strand breaks directly by attacking the DNA backbone (Vignard et al, 2013). This endangers the constitution of the whole genome, due to the fact that its interference with both strands carries a high potential for loss of genetic information.

Intercalating agents, like ethidium bromide and Benzo[a]pyrene (pollution, tobacco smoke), respectively attack the minor or major groove of the DNA double helix (Hess et al, 1997a; Yielding et al, 1979). Resembling a DNA nucleotide during S-phase, DNA polymerases create insertions while using it as template. This leads to frameshift mutations in daughter chromatids, and is in most cases much more severe regarding protein coding than missense mutations.

\subsubsection{UV induced bulky DNA lesions in human DNA}

One of the most extensive and omnipresent environmental mutagen is ultraviolet (UV) radiation (in particular UVA and UVB light, see Figure 1). Its energy creates a photochemical reaction and its efficacy depends on the wavelength. By energy absorption, in most cases adjacent pyrimidines are 
covalently linked together or with other molecules, e.g. in the presence of psoralen, leading to the formation of dimers (Hearst et al, 1984; Markovitsi et al, 2010). The maximum absorption of DNA bases is $254 \mathrm{~nm}$ (UVC), a wavelength, which is quantitatively absorbed by ozone and oxygen in the atmosphere but widely used in science because it ensures the highest rate of UV lesion formation (Rastogi et al, 2010).

Beside the spore photoproducts, specific for anhydrously living prokaryotes, the most commonly generated UV adducts in the DNA are cyclobutane pyrimidine dimers (CPDs; 75\%) and 6, 4 pyrimidine-pyrimidones (6-4PPs; 25\%) and their Dewar isomers (Rastogi et al, 2010; Yokoyama et al, 2012) (see Figure 4). The relevant main difference between these two lesions is the intensity of the kink they are introducing into the DNA backbone. 6-4PPs are characterized by a strong bending of about $44^{\circ} \mathrm{C}$ while CPDs show only about $9^{\circ} \mathrm{C}$ in rotation of the two bases towards each other (Kim \& Choi, 1995; Suquet \& Smerdon, 1993), which results in implications for the different modes of damage recognition (see chapter 1.3.4.1). A model of the chemical structure of the two DNA lesions is depicted in figure two.

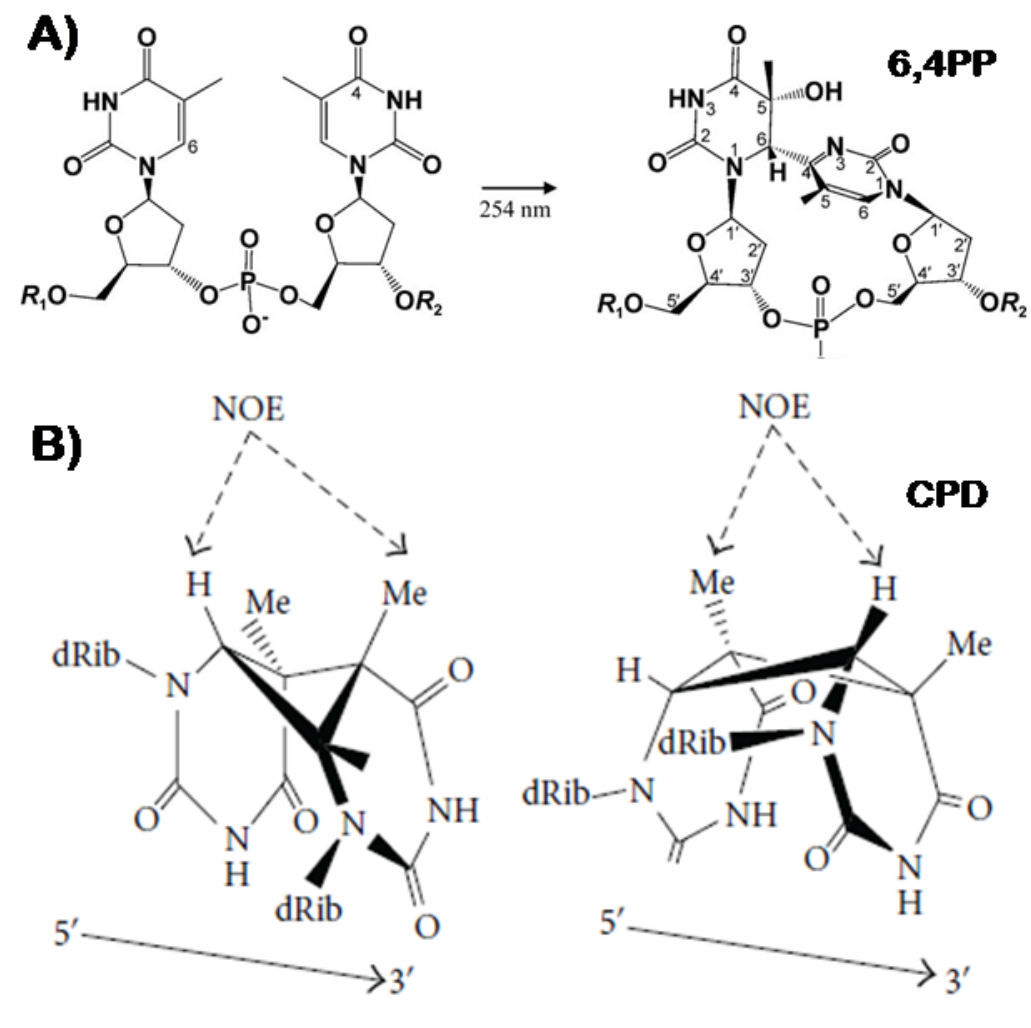

Figure 4: UV lesions of DNA UV light induces pyrimidine dimers between adjacent nucleotide of the same DNA strand.

A) 6-4PPs are developed by covalently linking the $\mathrm{C} 6$ atom of the $5^{\prime}$ base with the $\mathrm{C} 4$ of the 3' nucleotide. In this context it has been found that $\mathrm{T}-\mathrm{T}$ and $\mathrm{T}-\mathrm{C}$ are more abundant than C-T or C-C sequences. Due to extensive base skewing, 64PPs are known to introduce a strong bending in the DNA backbone.

B) CPDs arise by coalescence of the $\mathrm{C} 5$ and $\mathrm{C} 6$ positions of two neighboured pyrimidines mainly introducing small kinks into the DNA backbone (the 3' base is twisted around at the position of the $\mathrm{N}$-glycosidic bond).

Extensive base modifications of the DNA, introduced by alkylating and crosslinking agents as well as UV irradiation, are comparatively huge lesions, which distort the structure of the $\alpha$-helix and introduce kinks in the DNA backbone (so called bulky lesions). They are able to block polymerases and stall replication forks through this conformational change and, if left unrepaired, create mutations all over the genome during replication by the action of error-prone translesion synthesis 
(TLS) DNA polymerases (Branzei \& Foiani, 2010; Hanawalt, 1989; Zhang et al, 2000a; Zhang et al, 2000b) (see chapter 1.3.6). The UV signature, a C to T transition or CC to TT, is a typical mutation that has been found in $p 53$ genes of squamous cell carcinomas (Brash et al, 1991). Beside other ways of appearance, it is produced by the action of translesion polymerase $\eta$, which is error free upon CPDs, but can produce this UV mutation pattern "unintentionally" e.g. at the sites of uracil containing CPD (after deamination of cytosine) (Masutani et al, 2000). Other (indirect) influences on the UV signature are reviewed in (Ikehata \& Ono, 2011).

\subsection{Cells treat (UV) damage}

A lot of different DNA repair pathways have developed as an answer to the extensive amount of diverse mutagens (see chapter 1.2). This includes direct reversal (photolyase), Mismatch repair (MMR), Double strand break (DSB) repair, Base excision repair (BER) pathways etc. (reviewed in (Lombard et al, 2005)). This thesis concentrates on UV-dependent DNA damage and the responsible Nucleotide excision repair (NER) as well as translesional synthesis. Principally, all the repair pathways work in concert and are connected to the DDR in a cell cycle dependent manner (see chapter 1.3.1). The NER mainly works during G1-Phase (see chapter 1.3.2), whereas translesion synthesis is generally restricted to S-phase (see chapter 1.3.6).

\subsubsection{The universal DNA damage response (DDR)}

In order to guard the integrity of the genome and the genetic information content, evolution developed a rash of molecular mechanisms that are embraced by the term 'DNA damage response' (DDR). It is initiated by blocked polymerases or stalled replication forks and consists of a phosphorylation signal cascade (Giglia-Mari et al, 2011; Hoeijmakers, 2001). The DDR is in principle found among all species (Britt, 1996; Brown et al, 1999; DiRuggiero et al, 1997). Beside the so called SOS-response in prokaryotes it includes a broad range of DNA repair pathways, damage tolerance mechanisms, and cell-cycle checkpoint control steps in humans (Jackson \& Bartek, 2009; Opperman et al, 1996; Sutton et al, 2000).

Persisting DNA damage results in the coverage of ssDNA by Replication Protein A (RPA) (Wold, 1997). An additionally existing primer-template junction leads to subsequent recruitment of ATM (PI3K-like kinases ataxia-telangiectasia mutated) and ATR (ataxia-telangiectasia and Rad3-related) in mammals (Budzowska \& Kanaar, 2009; D'Amours \& Jackson, 2002; Ray et al, 2013). ATM and ATR phosphorylate the checkpoint effector kinases CHK2 and CHK1, respectively. This prevents replication of damaged DNA by activating p53 and p21, which in turn results in G1/S (ATM) or G2/M (ATR) checkpoint arrest to amongst others allow DNA repair (see Figure 5). 


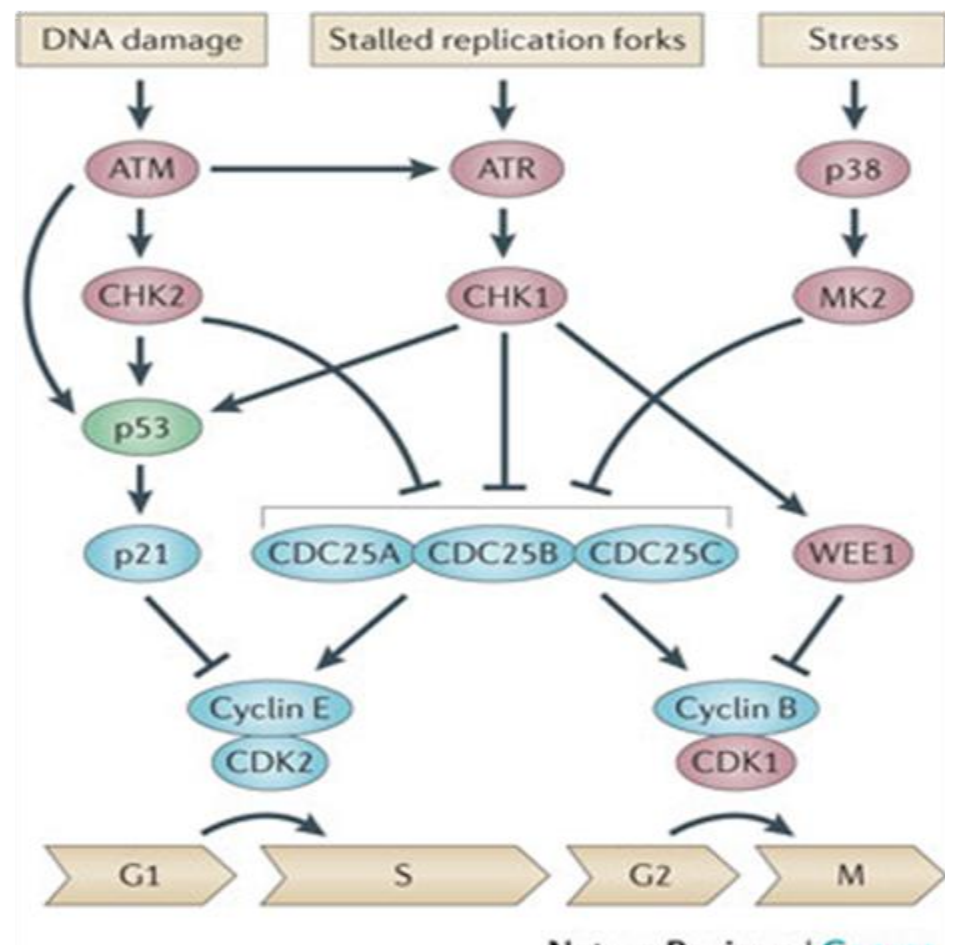

Figure 5: DNA damage response The DNA damage response (DDR) is mainly initiated by cellular signals like persisting DNA damage and stalled replication forks. The signaling cascade involves several kinases: sensors (ATM, ATR), adaptors (e.g. BRCA1 in UV response; not shown), transducer kinases $(\mathrm{CHK} 1,2)$ and effector proteins and pathways (cyclin B,E and CDK1,2 as well as cell cycle checkpoints). This signaling cascade ensures a chronological delay of cellular processes and proliferation to allow sufficient repair of DNA damages and lesions or promote (p53-dependent) apoptosis.

Nature Reviews | Cancer

Regarding the permanent mutagenic influence on the genome (see chapter 1.2) the DDR is an essential cellular process to secure genetic informational content and to avoid cellular malfunction and oncogenic transformation (Sertic et al, 2012).

\subsubsection{Nucleotide excision repair (NER)}

Some of the candidate genes, to date or in future, used as biomarkers for the cancer development or chemical tumor treatment perspectives are components of the Nucleotide excision repair (NER) pathway (Li et al, 2013a). The NER is the one and only error-free repair pathway in mammals which is able to repair bulky lesions (see chapter 1.2 ) in the DNA, characterized by a thermodynamically destabilized DNA duplex (Hess et al, 1997b). It was first discovered while observing the gap filling step utilizing unscheduled DNA synthesis (UDS, see chapter 3.5.2) in living cells in 1964 (Pettijohn \& Hanawalt, 1964; Rasmussen \& Painter, 1964). Since that time the understanding of this complex repair mechanism expended quickly, especially since it was associated with Xeroderma pigmentosum (XP) (see chapter 1.1.1), a genetic disorder with deficiency regarding NER and the removal of UV induced DNA adducts (see chapter 1.2.3) (Cleaver, 1968). Today, 50 years later, it is well known that it involves over 30 proteins (Le May et al, 2010). Some of them are conserved over the three major organism: archaea, prokarya and eucarya (Rouillon \& White, 2011).

There are extensive differences between prokaryotes and eukaryotes, especially humans (Morita et al, 2010). In contrast to the human set of proteins (>30), bacteria carry out the NER using only four proteins (UVR) (Truglio et al, 2006). The heterodimer UVR-AB constantly scans the DNA for damages and binds to them. Subsequently an exchange between UVR-A and UVR-C is triggered and the 
heterodimer UVR-BC, both subunits are carrying a nuclease activity, excises the fragment (Kisker et al, 2013). DNA polymerase II (often UVR-D in this context) synthesizes the new strand and seals the nick. Prokaryotic organisms also express photolyases performing direct reversal repair of UV induced DNA lesions (Zhang et al, 2013a). In eukaryotic organisms the protein products of the corresponding genes are probably converted into blue light receptors, whereas this repair pathway was evolutionary assumed by the NER (Kanai et al, 1997).

Human NER was reconstituted in vitro with 17 core components including the XP proteins XPA - XPG (Aboussekhra et al, 1995; Biggerstaff \& Wood, 1999; Shivji et al, 1999; Tapias et al, 2004). In contrast to other repair pathways the NER shows a broad bandwidth of substrates/lesions to be recognized. This DNA repair pathway is able to act in a cell-cycle independent manner and is both, a signal and an effector of the DNA damage response (DDR) (see chapter 1.3.1) (Giannattasio et al, 2010; Ray et al, 2013; Sertic et al, 2011; Sertic et al, 2012). The pathway is a sequential succession of molecular assembly steps carried out by several factors with special functions and features, which can be divided into three main stages: first the recognition of a DNA lesion, second the incision complex formation and DNA unwinding, and third the endonucleolytic incision around the lesion including gap filling, as it is briefly summarized in Figure 6 , see a detailed description of NER in chapter 1.3.4 (Scharer, 2013). 


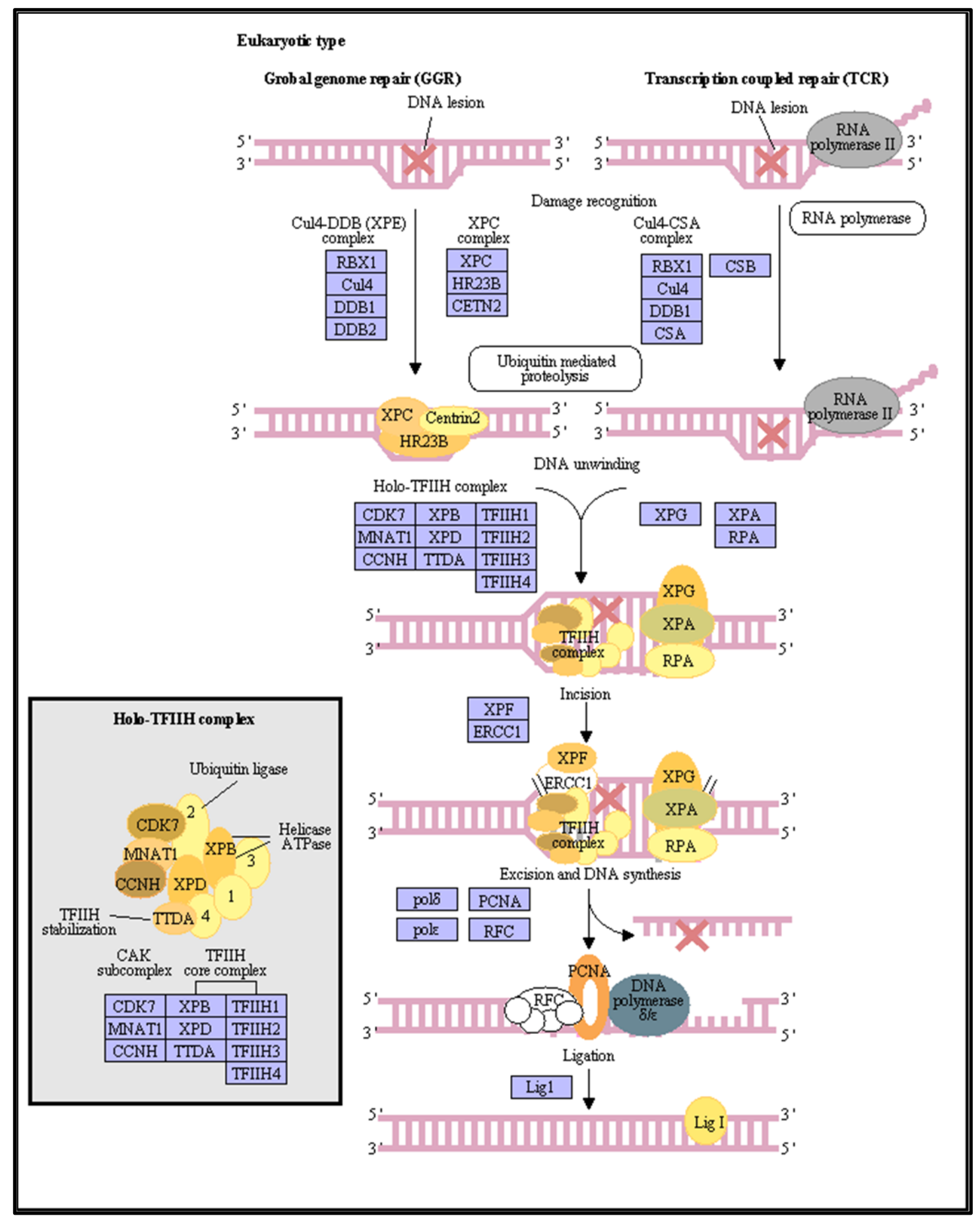

Figure 6: Nucleotide excision repair

The eukaryotic NER is subdivided into two coexisting damage recognition sub pathways, the transcription coupled repair (TCR) and Global genome repair (GGR). During GGR the XPC- and XPE complex serve as a lesion sensor, in the TCR pathway the RNA polymerase II together with the CUI4CSA complex and CSB detect the DNA adduct. Subsequently incision complex formation and DNA unwinding is performed by the recruitment of TFIIH (harbors helicase activity), XPG, RPA, and XPA. The endonucleases XPF-ERCC1 and XPG perform two incisions of the damaged strand upstream and downstream of the lesion. This results in the release of a DNA damage containing oligonucleotide and gap filling synthesis by action of RFC, PCNA and DNA polymerases $\delta / \varepsilon$ followed by the finalizing ligation by ligase I. (http://www.genome.jp) 
Introduction

\subsubsection{Damaged chromatin and Nucleotide excision repair}

The human genome is organized in 46 chromosomes and more or less tightly packed in either eu- or heterochromatin (Consortium, 2004; Oberdoerffer \& Sinclair, 2007). Before the core reaction of NER is discussed I want to take a closer look into the consequences of chromatin structures in the context of NER.

Euchromatin, the light variant of packaged nucleic acids, is the predominant form (about 92-99\%). It is often associated with actively transcribed genes (Consortium, 2004) (see chapter 1.3.4.1.2), because its structure ensures accessibility to the naked DNA for transcription factors and associated proteins. In contrast heterochromatin is the tightly packed variant of DNA and can be subdivided into two subclasses, constitutive and facultative heterochromatin. It has extensive implications for gene expression by silencing (Haaf \& Schmid, 2000; Johnson et al, 2013).

The molecular mechanism of NER in the context of chromatin is poorly understood and underrepresented in the NER research due to the fact, that the most results elucidated are produced utilizing in vitro assays with naked DNA (Tapias et al, 2004). However, it is well known, that UV induced lesions (CPDs, 6-4PPs) in heterochromatin are repaired much more slower than in unpacked DNA (Ura et al, 2001) because the NER needs chromatin relaxation for bubble unwinding (see chapter 1.3.4.3) and the incision process (see chapter 1.3.4.4). This is performed in a p53 dependent manner (Rubbi \& Milner, 2003). Especially in actively transcribed genes chromatin shows an inhibitory effect to the removal of CPDs (Bohr et al, 1985).

CPDs require recognition of both, the XPC-complex and the Cul4-DDB-complex, in future named the XPE-complex (see Figure 6) (Fitch et al, 2003b). The CUL4A E3 ligase of the XPE-complex needs DDB2 for recognition of UV lesions in chromatin, ubiquitinates XPC (see chapter 1.3.4.1.1), and (mono-) ubiquitinates the histones $\mathrm{H} 2 \mathrm{~A}, \mathrm{H} 3$ and $\mathrm{H} 4$, resulting in a quicker dissociation of nucleosomes in highsalt conditions due to a weaker interaction between histones and the DNA (Guerrero-Santoro et al, 2008; Itoh et al, 1999; Li et al, 1993; Sugasawa et al, 2005; Wang et al, 2006). Pines and co-workers further showed the interaction between PARP1, thus poly(ADP-ribosyl)ated UV-damaged chromatin, with DDB2.

Furthermore, the INO80 remodeling complex (INO80 and ARP5) was found to be essential for the removing of the relevant UV-induced lesions (see chapter 1.2.3) and that it binds the XPE-complex (DDB1) (Jiang et al, 2010). Hara and Sancar found that the removal of 6-4PPs, not CPDs, from nucleosomal DNA is stimulated by the SWI/SNF chromatin remodeling complex from Saccharomyces cerevisiae (Hara \& Sancar, 2003). Its human homolog, the BAF/PBAF-complex, stimulates the removal 
of both types of UVC-derived lesions in a histone H3 hyper acetylating fashion (Hargreaves \& Crabtree, 2011; Kuper \& Kisker, 2012).

This diversity suggests a great establishment and importance in the cellular functions of chromatin remodeling complexes. They are linked to NER via its damage recognition sensors of the Global genome repair (GGR) (see chapter 1.3.4.1.1). XPG and XPF were found to be associated with the CCCTC-binding factor (CTCF), a chromatin organizer, and trigger gene looping between promoter and terminator and the demethylation of activated genes (Le May et al, 2012) (see chapter 1.4.4). Furthermore, the PCNA interacting protein p21, which is a competitor of XPG, was associated with p300 recruitment and subsequent Histone acetyl transferase (HAT) activity, which is required for the chromatin remodeling prior to NER in response to UV (see chapters 1.4, 6.2).

The factor histone chaperone CAF-1 is believed to be responsible for the re-remodeling of chromatin after repair (Gaillard et al, 1996; Gaillard et al, 1997).

\subsubsection{NER steps in detail}

The NER is divided into different subsequent steps. The assembly of the proteins is also illustrated in Figure 6.

\subsubsection{Damage recognition}

The damage recognition step of the NER pathway can be divided into two sub pathways, the transcription coupled repair (TCR, see chapter 1.3.4.1.2) and the Global genome repair (GGR, see chapter 1.3.4.1.1) (Gillet \& Scharer, 2006; Hanawalt \& Spivak, 2008). It involves two completely different sets of proteins (see Figure 6) to initially detect the same bulky lesions in different cellular contexts using conserved $\beta$-hairpin structures (Kuper \& Kisker, 2012). However, they are still able to recruit the same factors, downstream of damage recognition (see chapter 1.3.4.2, 1.3.4.3), for further processing until the DNA lesions are removed in an error-free manner (see chapter 1.3.4.4).

\subsection{Damage monitoring during Global genome repair (GGR)}

The XPC-complex (XPC-HR23B-CETN2, see Figure 6) shows high affinity for both, damaged and undamaged DNA, acts as an universal damage sensor, and discovers various DNA lesions, while (strand wise) constantly scanning the genome for aberrations (Goosen, 2010; Reardon et al, 1996; Shell et al, 2013). HR23B stabilizes the XPC-complex and stimulates XPC activity during Nucleotide excision repair in vitro and CETN2 is involved in recruitment of XPA (see chapter 1.3.4.2) (Nishi et al, 2013; Sugasawa et al, 1996). Recognition of DNA damage triggers XPC-HR23B disruption and XPC stays left-behind at the site of DNA damage (Bergink et al, 2012). Due to the different degree in helix 
distortion by the diverse lesions, XPC shows a great affinity for 6-4PP but not for CPDs (see chapter 1.2.3) (Fitch et al, 2003b).

Recruitment of XPC to and efficient repair of CPDs, but not 6-4PPs, needs an additional factor, the XPE-complex (see Figure 6) (Fitch et al, 2003a; Sugasawa et al, 2005). DDB2 is the catalytic component of this complex which is known to be defective in XPE-patients and is able, due to its hydrophobic binding pocket, to bind to both types of UV-induced lesion (Scrima et al, 2008; Tang \& Chu, 2002; Yeh et al, 2012). The E3 ubiquitin ligase Cul4-RBX1 of the XPE complex, beside its function in chromatin relaxing (see chapter 1.3.3), poly-ubiquitinates XPC, thereby enhancing its binding affinity for the CPD lesion, and auto-ubiquitinates its own complex partner DDB2, preceding its degradation (Groisman et al, 2003; Takedachi et al, 2010).

XPC was also found to interact with the basal transcription factor IIH (TFIIH), which can be due to the fact that there is a handover of TFIIH between XPC and XPG during NER (see Figure 6). But an additional role of XPC in terms of transcription cannot be excluded to any extent (Drapkin et al, 1994; Schaeffer et al, 1993). Maybe, this also accounts for the observed self-downregulation of XPC mRNA levels in XPC patients (Schafer et al, 2013a).

\subsection{Damage recognition during Transcription coupled repair (TCR)}

In regions of actively transcribed genes (strands) the elongating RNA polymerase II itself serves as the initial damage detector if it is stalled (or back-tracked) at a DNA damage site and ensures rapid removal of bulky lesions in a complex with CSA, CSB and XPA binding protein 2 (XAB2) (see Figure 6) (Hoeijmakers, 2001; Nakatsu et al, 2000; Svejstrup, 2002). Although the nucleosome structure in transcribed regions is transiently opened, CSA is believed to play a role in euchromatin relaxing because it was found together with the XPE complex (Groisman et al, 2003). Also CSB, an ATPase and member of the SWI2/SNF2-family, shows chromatin remodeling functions in TCR context by inter alia recruiting the p300 Histone acetyl transferase (Fousteri \& Mullenders, 2008; Fousteri et al, 2006; Newman et al, 2006). Furthermore CSB seems to be one major factor in downstream recruitment and coordination of the other NER factors. It was shown to interact directly with the RNA polymerase II, CSA, and XPG (see chapter 1.4) (Henning et al, 1995; Sarker et al, 2005; Tantin et al, 1997). It is speculated that it serves as a kind of placeholder that keeps the transcription bubble opened before RPA and XPA are recruited (see chapter 1.3.4.2) (Hanawalt \& Spivak, 2008). Both CS-proteins are able to recruit XAB2 to sites of UV damage, which is essential for RNA recovery after UV irradiation (Kuraoka et al, 2008). XAB2 interacts with the polymerase, CSA, and CSB. It furthermore probably recruits XPA, and plays a pivotal role in TCR, transcription and mRNA splicing (Kuraoka et al, 2008; Lagerwerf et al, 2011; Park et al, 1995). 
Introduction

\subsubsection{Damage demarcation}

RPA is mainly active during S-phase, covering long single stranded stretches of DNA und thus preventing it from degradation, hydrolysis or endonucleolytic digestion (Wold, 1997). Furthermore persisting RPA is a major signal in the cellular process for ATR-dependent activation of cell cycle checkpoints (see chapter 1.3.1) (Cortez, 2005). However, in the context of NER RPA is recruited to sites of ongoing repair after XPC-HR23B and TFIIH are bound to the DNA (Overmeer et al, 2011). RPA has repair coordination and incision stimulating activity, and further seems to stabilize this ternary complex (XPC-TFIIH-RPA) by binding to the undamaged DNA strand (Aboussekhra et al, 1995; de Laat et al, 1998; Mocquet et al, 2008). Afterwards XPA is recruited to sites of lesion potentially by the interaction with the two large subunits of RPA (RPA70, RPA32) (Saijo et al, 2011). The interaction between RPA and XPG has been demonstrated by He and colleagues (He et al, 1995).

XPA has a higher affinity to kinked DNA than to lesion containing DNA and interacts with TFIIH, RPA,XPC-HR23B, DDB2, ERCC1, and PCNA (Scharer, 2013). XPA is a very crucial factor for functional NER and is believed to check the correct composition of the complex at the $5^{\prime}$ side of the bubble or maybe plays a role in identifying the lesion containing strand (Krasikova et al, 2010; Nouspikel, 2009). It is posttranslational acetylated (deacetylated by SIRT1, ensuring optimal NER (Fan \& Luo, 2010)) and its protein level is regulated by the circadian clock as well as the ubiquitin proteasome (Kang et al, 2011). It was further shown to be dominantly localized in the nucleus during G1- and G2-phase in dependency of p53 (Li et al, 2011a; Li et al, 2011b). But due to its protein size (31 kDa) one might speculate about an active export mechanism rather than an import mechanism, because proteins with a size of $<40 \mathrm{kDa}$ are able to perform sufficient passive diffusion through nuclear pores (Chatterjee \& Stochaj, 1998). However, during S-phase the main part of the XPA protein fraction remains in the nucleus, underlining its role in replication.

\subsubsection{The preincision complex (bubble formation)}

Whether the NER is initiated by the GGR or TCR, the next recruited essential factor for the preincision complex formation is the transcription factor IIH (TFIIH) (see Figure 6). It consists of two subcomplexes: the CAK-complex (CDK7 etc.) and the core-complex (p34, p44, p52, p62, TTDA, XPB and XPD), see Figure 7. 

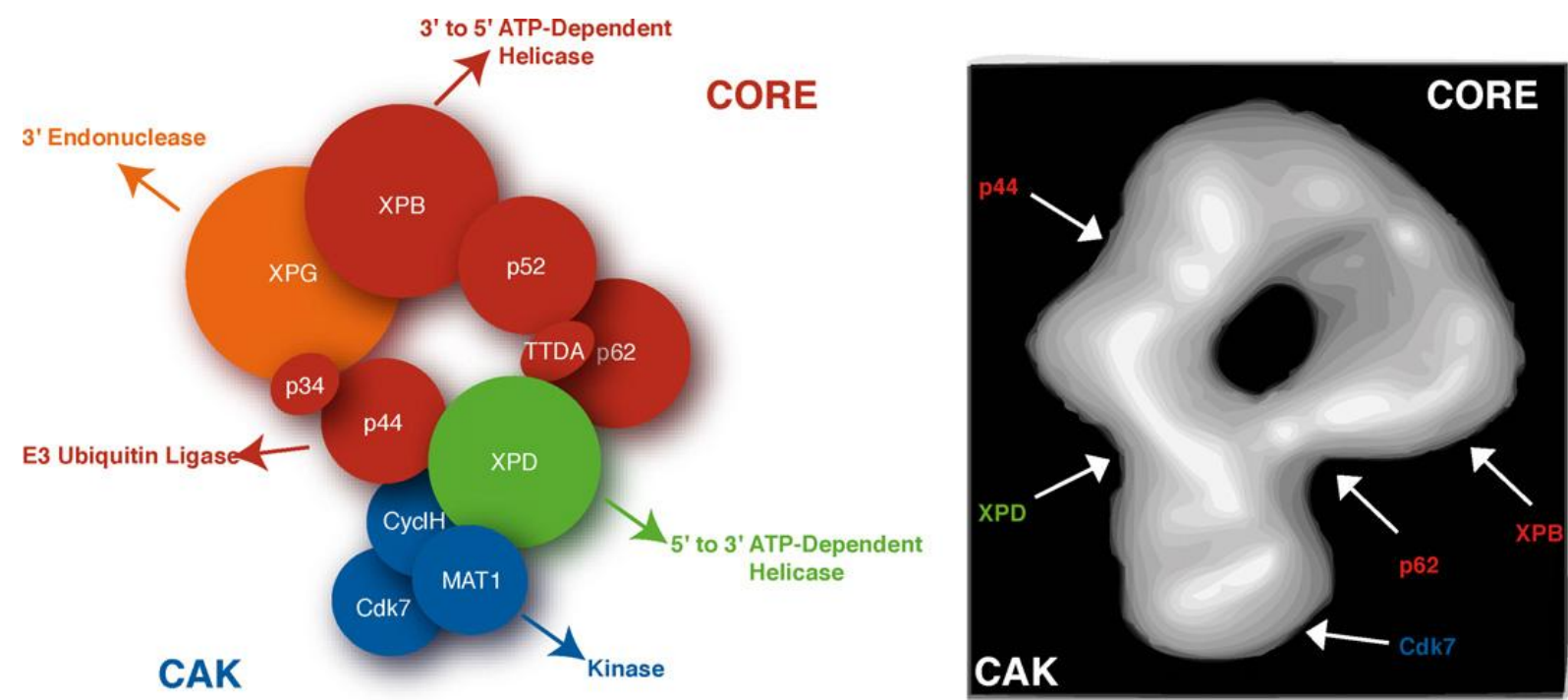

Figure 7: Transcription factor IIH (TFIIH)

TFIIH mainly consists of the helicase function bearing core complex (XPB, p52, TTDA, p62, p34, p44, and XPD), the CAK (Cyclin activated kinase) complex (Cdk7, Cycl H, Mat1) and of late XPG (see chapter 1.4) (Egly \& Coin, 2011). The molecular structure indicates a ring complex with kinase head.

XPD serves as a molecular bridge between those two subcomplexes and is also found in a free cellular CAK-XPD complex during mitosis (Chen et al, 2003; Compe \& Egly, 2012). XPG is designated the eleventh subunit of $\mathrm{TFIIH}$, because it was found together with the transcription factor and is believed to strengthens the association between both subcomplexes (Egly \& Coin, 2011; Ito et al, 2007; Mu et al, 1995). During GGR and TCR, TFIIH is recruited via direct interaction with XPC-HR23B or by XPA, respectively. (Park et al, 1995; Riedl et al, 2003)

In vitro footprinting experiments revealed that once XPC-HR23B is bound to a DNA lesion the double helix structure is slightly opened from -3 to +6 (nucleotide positions around the lesion) which is further extended ( -6 to +6 ) by arrival of TFIIH (Tapias et al, 2004). Human XPG arrives at the preincision complex in a DDB2-independent manner and is believed to be recruited after XPA binding or, in case of TCR, possibly by its CSB binding ability (Araujo et al, 2001; Bertani, 1951; Coin et al, 2008). Structural characterization of Saccharomyces cerevisiae repair complexes indicated for the displacement of Rad4 (XPC homolog) by Rad2 (XPG homolog) in dependency of TFIIH-presence (Lafrance-Vanasse et al, 2013). XPC is displaced by TFIIH which precedes the binding of RPA and XPA, which ensures the opened state of the bubble (Hanawalt \& Spivak, 2008; Tapias et al, 2004). This recruitment promotes CAK-dissociation from core-TFIIH, CAK is dispensable for functional NER (Arab et al, 2010; Coin et al, 2008). XPG's binding was shown to further extend the DNA melting and hence bubble formation (Tapias et al, 2004).

However, the CAK-complex is known to phosphorylate the CTD of the RNA polymerase II prior to elongation during transcription, possibly in a XPB-dependent manner (Egly \& Coin, 2011; Larochelle 
et al, 2012; Zhu et al, 2012). In the NER context, the CAK complex dissociates from the core complex, probably due to steric reasons, but surely prior to DNA melting and the anchoring of TFIIH to the DNA in an ATP-dependent manner (Araujo et al, 2000; Coin et al, 2008; Tapias et al, 2004). The two conserved helicases XPB $\left(3^{\prime}-5^{\prime}\right)$ and XPD $\left(5^{\prime}-3^{\prime}\right)$ carry the main catalytic activity of TFIIH during NER. XPB's ATPase activity [stimulated by the general TFIIH stabilizer p8 and potentially p52 (Compe \& Egly, 2012; Fregoso et al, 2007)], but not its helicase function as well as XPD's helicase and ATPase activity, are essential for NER and the preincision complex formation (Coin et al, 2007; Winkler et al, 2000). The C-terminal end of XPD and thereby the p44-XPD interaction is crucial for open complex formation (Coin et al, 1998a; Coin et al, 1998b; Dubaele et al, 2003). Furthermore, XPD has a conserved damage verification function by an iron-sulfur cluster channel, which is too tight for a helix-distorted single stranded DNA, e.g. by a bulky lesion (Mathieu et al, 2013; Naegeli et al, 1992). This fact is nowadays seen as an important trigger to complete preincision complex assembly with the recruitment of RPA, XPA and XPG (Scharer, 2013).

After anchoring TFIIH to the site of DNA damage by XPA and RPA, the two endonucleases XPF/ERCC1 $\left(3^{\prime}\right)$ and XPG (5') are recruited (see chapter1.3.4.4).

\subsubsection{Dual incision complex and gap filling}

The structure specific endonuclease XPG (see chapter 1.4) is strongly associated with basal transcription and the TFIIH complex. It is recruited to the DNA lesion as the first of both endonucleases, probably together with or prior to TFIIH, but surely in dependency of ATP and functional TFIIH (Evans et al, 1997; Harrington \& Lieber, 1994; Hohl et al, 2003; Sarker et al, 2005; Scharer, 2008a; Wakasugi \& Sancar, 1998; Zotter et al, 2006). Furthermore, arrival of XPG opens the repair bubble until it is completely opened from position -19 to +8 (Tapias et al, 2004). The second endonuclease, the heterodimer XPF-ERCC1, whereas XPF offers the catalytic activity, is recruited via the interaction between ERCC1 and XPA (Enzlin \& Scharer, 2002; Li et al, 1994). The assembly of the two nucleases occurs in a directed fashion due to the polarity and direction of the preassembled components, especially RPA (de Laat et al, 1998). The catalytic activity of both endonucleases is revealed in the only one formation possible, due to the tight opening of the bubble, in which XPF/ERCC1 is bound to the DNA at the 5' side and XPG at the 3' side of the lesion (O'Donovan et al, 1994a). 


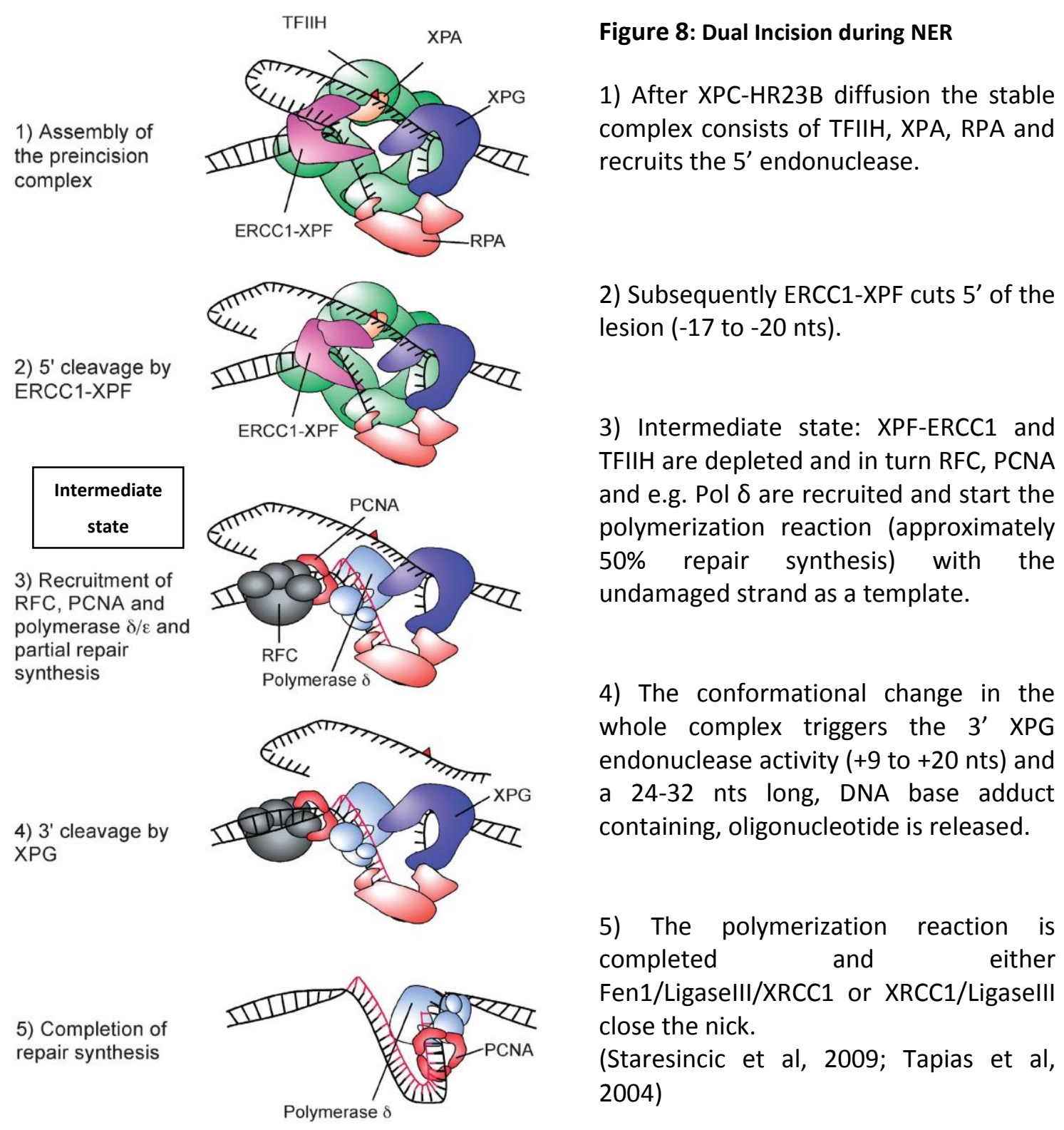

The first incision is performed 5' of the DNA lesion by XPF/ERCC1 in dependency of XPG's presence (see Figure 8) (Staresincic et al, 2009). Also the dephosphorylation at S751 of XPB, most probably induced by PP2A, is a prerequisite for XPF-ERCC1 incision, but not for DNA unwinding (Ariza et al, 1996). This secondary modification is restricted to the NER context, because the S751, in vivo and in vitro created, phosphorylation status (by casein kinase II) leaves TFIIH transcriptional activity unaffected (Coin et al, 2004). The first endonucleolytic cut creates a lesion containing single stranded DNA flap with a free $5^{\prime}$ end and an unbound $3^{\prime}$ hydroxyl group at the dsDNA-ssDNA junction. The latter is part of a primer for the elongation by DNA polymerases (e.g. $\delta$ or $\varepsilon$ and $\mathrm{k}$ ), able to synthesize according to the undamaged template strand (Lehmann, 2011; Ogi et al, 2010).

After the first incision took place, PCNA (see chapter 1.3.5), the clamp loader RFC, and a polymerase are recruited. The arrival of PCNA was shown to be in dependency of RPA- and XPG-presence, furthermore the catalytic activity of XPF is required (Mocquet et al, 2008; Staresincic et al, 2009). The 
repair synthesis immediately starts prior to the second cut by XPG in a RFC-dependent manner (Mocquet et al, 2008; Staresincic et al, 2009). The clamp loader RFC, which is needed for Pol $\delta$, but not for PCNA recruitment (PCNA self loading), is in turn recruited to the NER incision complex in a 5' incision- and RPA-dependent manner and facilitates XPF release (Mocquet et al, 2008; Overmeer et al, 2010). This probably ensures avoidance of persisting single stranded gaps which in turn would lead to checkpoint activation (see chapter 1.3.1) or maybe Exo1 recruitment, like it was shown in yeast and human cells (Giannattasio et al, 2010; Sertic et al, 2011) (see chapter 1.5.1.2). For their activation, translesion polymerase $\mathrm{k}$ requires ubiquitinated PCNA, polymerase $\delta$ requires RFC and PCNA, and polymerase $\varepsilon$ requires the modified version of RFC (Ctf18 containing) (Lehmann, 2011; Ogi et al, 2010). RPA is released from the undamaged strand as soon as Pol $\delta$ is recruited, most likely due to action of the polymerase itself (Mocquet et al, 2008). This intermediate state is an ongoing field of investigation, and is the key question of this thesis (see chapter 1.6).

However, XPG (see chapter 1.4) performs the second cut on the $3^{\prime}$ side and the lesion containing oligonucleotide (24-32 nts) is released together with XPG and TFIIH, maybe because the lesion still stucks in XPD's DNA binding pocket (see chapter 1.3.4.3) (Hu et al, 2013; Kemp et al, 2012; Moggs et al, 1996). During the intermediate state a 'cut-patch-cut-patch' mechanism is postulated for the action of both endonucleases, in reference to early investigations on excision repair in bacteria (Hanawalt \& Haynes, 1967; Staresincic et al, 2009).

After TFIIH's binding to ATP the excised oligonucleotide is released and bound by RPA, that is able to protect the oligo from degradation to a certain extent, but will although lead to its slow degradation to 6- or 7-mers with a proportion of 2-mers (mean 3.7), maybe by the action of 5'-3' exonucleases (Galloway et al, 1994; Kemp et al, 2012; Weinfeld et al, 1986). The created short ssDNA fragments should be able to activate ATM, linking the excision process to the general DNA damage response (see chapter 1.3.1) (Jazayeri et al, 2008). However, the suggestion that all dimers will be degraded to 2-mers at the end, because this would make excretion more easily, lacks its proof. Only very little is known about the export and excretion of the dimers, although they were detected in urine (Ahmad et al, 1999; Cooke et al, 2001). Furthermore, short oligonucleotides, especially 2-mers, were detected increase the melanin production in living cells (Eller et al, 1997).

Finally, in non-dividing cells DNA ligase III, XRCC1 and Pol $\delta$ and in replicating cells either Pol $\varepsilon$ and DNA ligase I or DNA ligase III, XRCC1 and Fen1 (see chapter 1.5.1.1) are required for completion of repair synthesis and nick sealing (Moser et al, 2007). 
Introduction

\subsubsection{Proliferating Cell Nuclear Antigen (PCNA) acts during and after dual incision}

Proliferating Cell Nuclear Antigen (PCNA) was discovered in 1978 only in nuclei of dividing cells, acts as a homotrimer and comprises ring structure as depicted in Figure 9, and ten years later it was established as an essential factor for replication (Bravo \& Celis, 1980; Miyachi et al, 1978; Prelich et al, 1987a; Prelich et al, 1987b). Until today, PCNA is an intensively studied protein, principally well known regarding its mode of action (DNA sliding clamp), structure, and conservation (Krishna et al, 1994a; Krishna et al, 1994b; Warbrick, 2000). PCNA is furthermore linked to other pathways than replication, e.g. DNA repair (NER, BER, MMR, DSB repair) (Jonsson \& Hubscher, 1997; Kelman, 1997; Kelman \& Hurwitz, 1998). Due to sequence homologies of the conserved PCNA interacting peptide (PIP) motif for PCNA interaction (see chapter 1.4.2), a lot of binding partners (p21, Fen1 (see chapter 1.5.1.1), Cdc27/p68 (Pol $\delta$ subunits), XPG (see chapter 1.4), MCMT, WRN, RFC1, DNA ligase 1, and Ung2) were identified (Warbrick, 2000). The consensus sequence of the PIP-box is [QXXhXXaa], Q is glutamine, $\mathrm{h}$ is a moderately hydrophobic residue, $\mathrm{a}$ is an aromatic side chain (normally FF), and $\mathrm{x}$ is any residue. This thesis concentrates on some of the interactors of PCNA, having an impact on the recent question (see chapter 1.6).

PCNA acts as a homotrimer in eukaryotes and every monomer has three potential peptide interaction sites, with a conserved hydrophobic pocket, which enables PCNA to interact with diverse binding partners (at once), e.g. several polymerases (Indiani et al, 2005; Krishna et al, 1994b). A major PCNA binding protein is its inhibitor, the CDK7 inhibitor, p21 (Warbrick et al, 1995). P21 inhibits both bindings of PCNA to either DNA or to polymerases during replication, and furthermore regulates PCNA during DDR (see chapter 1.3.1) (Podust et al, 1995; Shivji et al, 1998). XPG was found to inhibit p21 binding of PCNA (Mocquet et al, 2008; Warbrick, 2000; Warbrick et al, 1997). However, the residence time of PCNA at areas of ongoing DNA synthesis in replication or repair context is significantly different and enhanced at damaged site in an ubiquitination dependent manner (Essers et al, 2005). Furthermore Essers and colleagues showed a reduced resident time of an ubiquitinationdefective PCNA mutant, linking its ubiquitination rather to the stabilization of TLS polymerases at UV lesion than to its recruitment. This is consistent with the observation of direct interactions between PCNA and TLS polymerases, as well as ubiquitin independent recruitment (see chapter 1.3.6).

PCNA interacts with several eukaryotic polymerases $(\delta, \eta, \zeta)$ on recombination DNA intermediates (Li et al, 2013b). The processivity of Pol $\delta$ increased after p68 (Pol $\delta$ subunit) phosphorylation mediated PCNA recruitment and it was long thought, that the translesion polymerase $\eta$ is recruited to DNA damage sites in a PCNA ubiquitination (K164) dependent manner (see chapter 1.3.6) (Rahmeh et al, 2012; Stelter \& Ulrich, 2003). Therefore secondary modifications, like ubiquitination, sumoylation, acetylation and phosphorylation, all leading to diverse cellular events, were an extraordinary 
element of PCNA biology (Ulrich \& Takahashi, 2013). I will focus on the conserved K164 monoubiquitination- dependent recruitment of translesion polymerases (Stelter \& Ulrich, 2003). This highly conserved lysine of the human PCNA is either Rad6-Rad18 (E2-E3, respectively) dependently mono-ubiquitinated, or further poly-ubiquitinated by the Ubc13-Mms2 complex (E2) via the en bloc attachment of up to four K63 ubiquitin molecules at once (Hoege et al, 2002; Masuda et al, 2012). The latter is associated with template strand switching (Ulrich \& Takahashi, 2013). Rad18 is recruited to PCNA in a RPA (RPA $\mathrm{R}_{74}$ ) dependent manner (Yang \& Woodgate, 2007).

The multi subunit complex replication factor $\mathrm{C}(\mathrm{RFC})$ loads PCNA on the template in an ATPdependent manner (Mossi \& Hubscher, 1998). Clamp loader are highly conserved structures, but the interaction with PCNA is not (Uhlmann et al, 1997). However, Ligase I and RFC share one binding motive in a conserved fashion, what raises the possibility of a displacement by each other or simultaneous binding of two different monomers of PCNA (Mossi \& Hubscher, 1998). The RFC complex acts as a highly processive compartment of the replication machinery, whereas the polymerase is distributive and even PCNA's processivity is dependent on the presence of RFC, which remains at the $3^{\prime}$ terminus during elongation (Masuda et al, 2007). RFC is responsible for the persistence of PCNA at sites of local UV damage (Overmeer et al, 2010). Furthermore, human PCNA was found to be sumoylated at K164 in a DNA and DNA damage independent manner, but in dependency of RFC (Gali et al, 2012). Gali et al. further showed that sumoylation rather has an inhibitory effect and prevents double strand break initiated homologues recombination (HR) and the formation of double strand breaks at stalled replication forks. These facts indicate great importance of the RFC-PCNA interaction.

In the context of NER, the RFC arrival at damaged (immobilized) DNA was facilitated by RPA and triggers XPF-release (Mocquet et al, 2008). It is assumed, that RFC and XPG concomitantly recruit and stabilize PCNA, what in turn triggers RPA and XPG release, Pol $\delta$ recruitment, and subsequent DNA synthesis. Thus, PCNA recruitment demands on the $5^{\prime}$ incision by XPF/ERCC1. XPG is acetylated in vitro in a p300 dependent fashion, and this attachment is inhibited by PCNA and is in turn dependent on the interaction between p21 and PCNA (Tillhon et al, 2012). XPG is known to inhibit p21 binding to PCNA (see chapter 1.4). However, the cyclin-dependent kinase (CDK) PCNA inhibitor p21, which degradation promotes PCNA-dependent DNA repair, was also found to inhibit efficient ubiquitination of PCNA (via CDK, no direct interaction) (Soria et al, 2006). This fact raises the question about a role of ubiquitin in DNA repair beside translesion synthesis. The interaction between XPG and PCNA is extensively reconsidered in the respective section (see chapter 1.3.6) and in the next chapters (see chapters 4 and 5 ). 
PCNA binds amongst others Fen1, a factor involved in long patch BER and Okazaki fragment maturation, and stimulates its nucleolytic activity up to 50-fold (see chapter 1.5.1.1) (Li et al, 1995; Wu et al, 1996). Fen1, like XPG, competes with p21 for binding via the conserved PIP box (Gary et al, 1997; Warbrick et al, 1997). Further, PCNA-Fen1 interaction was identified as the trigger leading to Fen1 cleavage in a defined in vitro system (Craggs et al, 2013).

Another binding partner of PCNA is the replicative DNA ligase I in G1- and S- phase in a cell cycle regulated dephosphorylation dependent manner (S66 of Ligl), linking PCNA and the dephosphorylation apparatus (Montecucco et al, 1998; Rossi et al, 1999). XRCC1 directly interacts with PCNA only in S-phase (Fan et al, 2004).

PCNA interacts with several eukaryotic polymerases $(\delta, \eta, \zeta)$ on recombination DNA intermediates (Li et al, 2013b). The processivity of Pol $\delta$ increased after p68 (Pol $\delta$ subunit) phosphorylation mediated PCNA recruitment and it was long thought, that the translesion polymerases are recruited to DNA damage sites in a strictly PCNA ubiquitination (K164) dependent manner (see chapter 1.3.6) (Rahmeh et al, 2012; Stelter \& Ulrich, 2003).

PCNA acts as a ring shaped homotrimer and should be able to bind more than one reaction partners at once. During this thesis, and especially for the drawing of the model (see Figure 27), the "revolver barrel" picture of PCNA is taken as a basis, like it was proposed before (Yang \& Woodgate, 2007). PCNA, stably attached to the DNA (or in a cycle of recurring loading and unloading) by RFC, is able to deliver three reaction partners/proteins/protein complexes ("bullets") to sites of ongoing replication or DNA repair at the same time, and is able to exchange the several enzymes very quick during the reactions. This includes nucleases (XPG, Fen1, and Exo1), several polymerases, DNA ligases and other scaffold proteins (Rev1, XRCC1) (see chapters 1.3.6, 1.4, and 1.5).

\subsubsection{Translesion synthesis}

Processive replicative polymerases are elongating with a rate of 1000 nucleotides per second, and are normally unable to incorporate nucleotides opposite of a DNA base adduct (with a few exceptions, e.g. Poll) (Lehmann et al, 2007; Pages \& Fuchs, 2002). In contrast, low fidelity translesion polymerases are able to use damaged DNA as a template and incorporate nucleotides in an accurate or mutagenic fashion, due to a wider nucleotide binding pocket. However, the frequency of wrong incorporation of a nucleotide by a translesion polymerase, acting on an undamaged template, was estimated 1:30 for Pol $\eta$ (Masutani et al, 2000).

The $\mathrm{Y}$-family polymerase $\eta$ is encoded by the $P O L H$ gene and in the eighth complementation group of genetically defective XP-Variant patients with mutations mostly compromising the DNA binding 
ability of Pol $\eta$ (Biertumpfel et al, 2010; Gratchev et al, 2003) (see chapter 1.1.1). XP variant patients exhibit all major symptoms of the XP disease (sunlight induced pigmentation changes and a higher incidence of tumor formation of sun exposed areas), indicating a general role for translesion synthesis in preventing cells from malignant transformation (see chapters 1.2, 1.3 \&).

Like XPG (see chapter 1.4), Y-family polymerases normally comprise three conserved domains postulated to be important for PCNA interaction (see chapter 1.3.5), the PIP-box and two ubiquitin binding domains (zinc-finger UBZ and hydrophobic UBM) (Bienko et al, 2005; Freudenthal et al, 2010; Ulrich \& Takahashi, 2013). The simultaneous binding to PCNA via both ubiquitin binding domains (in a flexible arm of the molecule) is expected.

Pol $\eta$ is able to bypass CPDs, but not 6-4PPs (see chapter 1.2.3). 6-4PPs are probably mostly repaired by Translesion (TLS) polymerases Rev1 and, in particular Pol ל, like it was seen in mouse embryonic fibroblasts (Temviriyanukul et al, 2012). A clear evidence in human cells is missing, but Rev1 seems to have a great influence on PCNA ubiquitination dependent post UV survival (McCulloch et al, 2004; Qin et al, 2013; Yao et al, 2001). Pol $\eta$ was able to bypass TT dimers with high efficacy and to incorporate two adenines in an accurate fashion (Masutani et al, 2000). The error prone mutagenity arises from bypassing other types of lesions like AP-sites, 8-oxoguanine, and benzo[a]pyrene with varying incorporations (mostly A/T mutation pattern, which was for instance found in hyper mutated regions of immunoglobulin genes) and also by the creation of deletions (Faili et al, 2004; Franklin et al, 2004; Zhang et al, 2000a).

The recruitment of TLS polymerases (e.g. Pol $\eta$ ) to sites of DNA damage is strongly dependent on PCNA-mono-ubiquitination (Bienko et al, 2005). Contrary, during ICL repair TLS is performed in a PCNA ubiquitination independent manner (Hendel et al, 2011; Hicks et al, 2010) (see Figure 9). In this context, inhibitors of the PIP-box of TLS polymerases were sufficient to decrease PCNA-mediated synthesis after cisplatin treatment (Actis et al, 2013). This can be explained by the existence of direct non-canonical interactions between PCNA and translesion polymerases, e.g. three hydrogen bonds between Pol $\eta$ and PCNA (Leu711-Gln125, Leu704-His44, Gly700-Ile255) (Hishiki et al, 2009).

Rev1 is special among translesion synthesis polymerases and possesses the unique ability to use dCMP (Yang \& Woodgate, 2007). Furthermore it comprises a BRCT-domain, which was shown to facilitate PCNA-binding as well as two UBM domains and most probably fulfills a structural rather than catalytic role (Pustovalova et al, 2013). As it was demonstrated in human and murine cells, beside its ability to perform TLS, Rev1 is able to act as a scaffold, coordinating other translesion polymerases ( $\eta, \zeta, \mathrm{K}, \mathrm{l}$, all share a common Rev1 interacting region) and their association with PCNA (Hendel et al, 2011; Yang \& Woodgate, 2007) (see Figure 9). The regulation of the different 
polymerases is not understood yet and discussed in detail in the actual review from Sale, Lehmann and Woodgate (Sale et al, 2012).

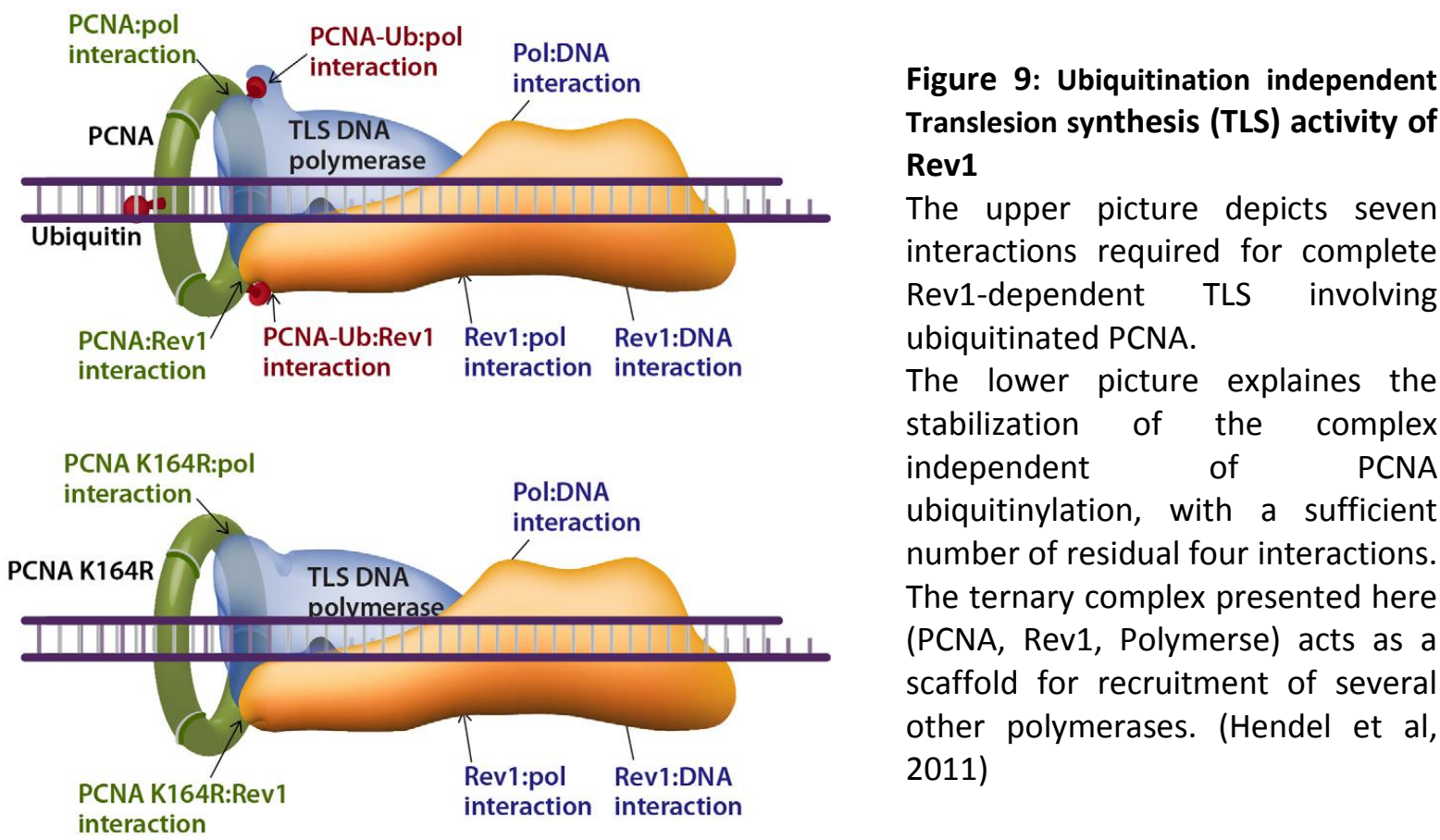

Furthermore Essers and colleagues showed a reduced resident time of an ubiquitination-defective PCNA mutant, linking its ubiquitination rather to the stabilization of TLS polymerases at UV lesion than to its recruitment, consistent with the observation of direct interactions between PCNA and TLS polymerases (see chapter 1.3.5) (Essers et al, 2005).

The C-terminal domain of Pol $\eta$ binds to both, Rad18 and PCNA, and promotes ubiquitination, independent of its nuclease function. This C-terminus of Poln is homologues to the XPG N-terminus (Durando et al, 2013; Fagbemi, 2012). Pol $\eta$ expression is regulated by RPA and p53 in response to UV, noteworthy Pol $\mathrm{k}$ was recruited in case of a catalytically defect mutant of Pol $\eta$.

However, the interaction between Pol $\eta$ or Rev1 and ubiquitinated PCNA is much stronger compared to PCNA alone and probably a recruitment enhancing signal as well as an accessory factor for polymerase switch (Yang \& Woodgate, 2007).

\subsection{Detailed description of XPG}

XPG was discovered as an NER-associated and XP-causing enzyme in the late 1970s by cell-fusing experiments and later by complementation studies using cloned cDNA (Keijzer et al, 1979; Scherly et al, 1993). It was first expressed and isolated as recombinant protein in Sf9 cells after polyhedrosis virus infection and identified as magnesium-dependent single stranded endonuclease (O'Donovan et al, 1994b). Moreover XPG is a structure specific endonuclease, a member of the Flap endonuclease 
family (Fen1- family) (see chapter 1.5.1.1) and responsible for the 3' cut during NER (see chapter 1.3.2) (Lieber, 1997; O'Donovan et al, 1994a). XPG is an essential protein in both pathways, GGR and TCR (see chapter 1.3.4.1) and is, additionally involved in other repair pathways and in association with TFIIH also involved in basal transcription (Scharer, 2008a). Moreover, the length of XPG protein accounts for the XPG/CS phenotype, instead of simply XP, like no other factor (see chapter 1.1.2) (Ito et al, 2007). Beside its major function, during the last years, XPG's contribution to more and more other cellular processes was identified (see chapters 1.4.3, 1.4.4).

\subsubsection{XPG patients demonstrate multiple functions of XPG}

To date 21 Xeroderma pigmentosum patients with 28 disease-causing mutations were identified and assigned to the complementation group G (XP-G) (Schubert et al, 2013; Soltys et al, 2013). The clinical spectrum (see Figure 2) shows a very diverse picture with eight patients exhibiting XP, three XP patients with late onset CS and the majority of ten patients suffers from severe XP/CS (Schafer et al, 2013b). Among the patients with known genotypes people with (mild) simple XP phenotype mostly still possess about at least one full-length allele with a point mutation in one of the two endonuclease domains (see Figure 10) and thus exhibit residual repair capacity (Scharer, 2008b). The fraction of patients with additional late onset CS symptoms is of special attention, because it includes two patients (XP3BR, XPCS1BD) with more or less full-length splicevariant expression, protecting the patients from at least the early onset of CS symptoms, because their dominant alleles express only truncated (a) version(s) of the protein (Lalle et al, 2002; Thorel et al, 2004). It is quite well established, that without these full-length isoforms, the truncated XPG proteins would not be capable to maintain interaction between XPG and TFIIH, what causes combined XPG-CS with an early onset of CS symptoms (see chapter 1.1.2) (Emmert et al, 2002; Ito et al, 2007; Nouspikel et al, 1997). The genotypes of the most XPG/CS patients supports this suggestion (Scharer, 2008b), but there are a few exceptions. The patient XPCS4RO has a point mutation in the $\mathrm{N}$-domain leading to an amino acid substitution (P72H) and subsequent destabilization (or degradation) of the (full-length) protein (Zafeiriou et al, 2001). One of the most recent reports accounts two severely affected XP/CS patients with at least one full-length allele but with a point mutation in the I-domain (see Figure 10) resulting in a stable expression but a defective NER and impaired TFIIH (XPD) binding (Schafer et al, 2013b). However, the majority of the patients of the rare XPG complementation group are hardly concerned with classical XP and additional CS symptoms, punctuating the influence of XPG to the general transcription machinery (see chapter 1.4.3).

XPG mice, with the corresponding E791A endonuclease mutation, showed a classical XP phenotype with UV hypersensitivity (Tian et al, 2004). Knockout mice with a truncation mutation (nts264-380) display a severe XP/CS phenotype with growth retardation and early death (three weeks) (Harada et 
al, 1999). This supports the strict genotype-phenotype correlation between the constitution of XPG and the clinical consequences.

Noteworthy and with respect to the clinical reference, SNPs of XPG were investigated regarding the possible predictive value for cancer progression and chemotherapeutic efficacy. One study showed a significant correlation between the NSCLC (non small cell lung carcinoma) and rather the CT+TT than CC genotype of rs22961471 for the therapeutic prognostic value (Yuli et al, 2013). Another study correlated the polymorphisms in the XPG gene with an 11 times increased mortality rate of melanoma patients (Li et al, 2013a).

\subsubsection{Established functional domains of XPG}

The XPG/ERCC5 gene is located at chromosome position 13q32.1-q33.1 (Takahashi et al, 1992) and encodes for an 1186 amino acid (133kDa) protein (Dunand-Sauthier et al, 2005) with several domains (see Figure 10).

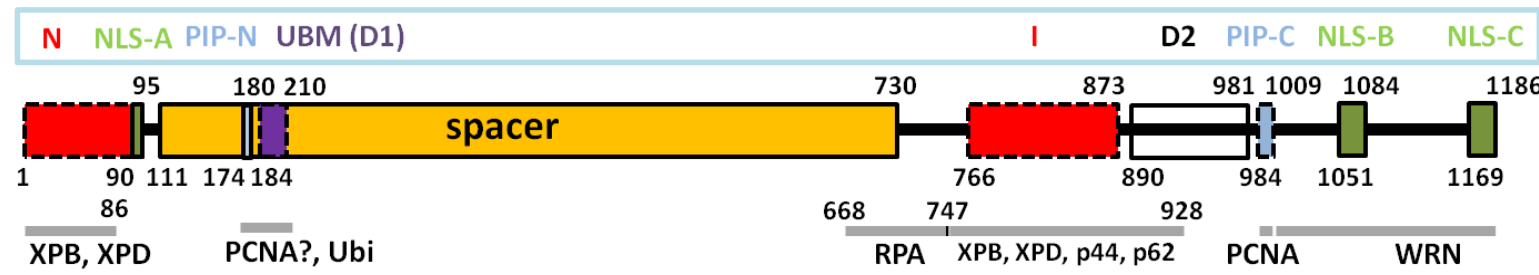

\section{Figure 10: Domains of XPG}

The box names are depicted in the cyan rectangle (Red boxes = endonucleolytic $\mathrm{N}+$ I domains, green boxes $=$ nuclear localization signals (NLS), blue boxes $=$ PCNA interacting peptides (PIP), purple box $=$ ubiquitin binding domain (UBM, also referred as the D1 domain), yellow box = spacer, black box = conserved D2 domain). The grey lines indicate interactions with several binding partners of XPG.

XPG is a structure (junction) specific endonuclease that binds to disturbed dsDNA via interactions with the phosphate backbone on the face of the helix (Hohl et al, 2003) (see Figure 11, additionally se Figure 13). The endonuclease activity is assigned to the two-tailed highly conserved $\mathrm{N}$ - and I-boxes, which are separated by a spacer (Harrington \& Lieber, 1994) (see Figure 12). These two domains contain essential residues for the endonuclease activity of XPG (e.g. D77, E791, D812) and are proposed to associate with each other in the quaternary structure (XPG plus dsDNA) (Constantinou et al, 1999; Mueser et al, 1996; Wakasugi et al, 1997). During this study, the endonuclease defective E791A and D77A mutants were constructed by introducing point mutations leading to (as indicated) amino acid (aa) exchanges at this sites. 


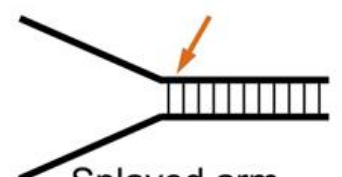

Splayed arm
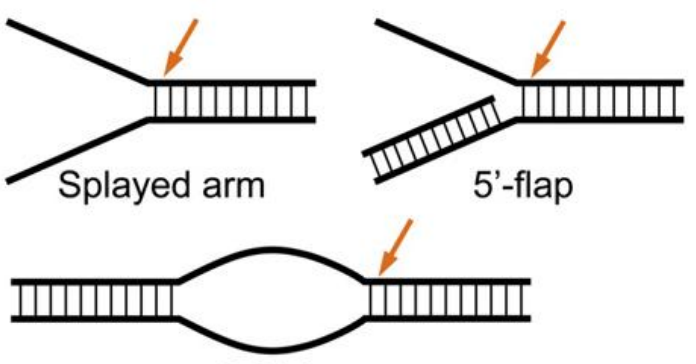

Bubble
Figure 11: Structures cleaved by XPG The endonuclease XPG cuts one strand of dsDNA with splayed arm structures, free $5^{\prime}$ ssDNA flaps and bubble structures (indicated by orange arrows). (Cloud et al, 1995; Fagbemi et al, 2011)

Three nuclear localization signals (NLS) were identified with varying activity (NLS-A $=40 \%$, NLS-B \& -C $=100 \%$ ) (Knauf et al, 1996). The N-terminal NLS-A with 40\% activity was sufficient to transport truncated XPG variants into nuclei (Lehmann, 2013).

The spacer region is a 600 aa long DNA patch with highly acidic residues (especially in D1), which supplies the substrate specifity for bubble structures to XPG (Hohl et al, 2003; Sarker et al, 2005; Scherly et al, 1993). It binds the TFIIH-components XPB and XPD (residues 1-377) (Dunand-Sauthier et al, 2005; lyer et al, 1996; Thorel et al, 2004). Hybrid enzyme constructions revealed, that Fen1 (see chapter 1.5.1.1) with the XPG spacer between the $\mathrm{N}$ - and I- domains showed features of both nucleases, Fen1 and XPG, and was able to cut bubble and flap structures and partially complement XPG-deficient cells in vivo but not in vitro (Hohl et al, 2007).

Furthermore, the spacer region contains a small N-terminal PIP domain (found in several XPGs of different species) and an evolutionally conserved ubiquitin binding domain (UBM) (Hofmann, 2009). The PIP-N domain was mutated at the essential proline P179A and the ubiquitin domain with two point mutations at the highly conserved residues L196 and P197 to alanine. The latter two have previously been shown to completely abolish ubiquitin binding in the same fashion like a deletion mutant (Fagbemi, 2012).

The domains D1 \& D2 are highly conserved in higher eukaryotes (drosophila, xenopus laevis, mouse, human) but not in S. cerevisiae and not in S. pombe suggesting a differential role of XPG in these organisms (Houle \& Friedberg, 1999).

Furthermore, interactions of XPG with RPA, CSB, PCNA, and WRN were reported (see chapter 1.3.5) (Gary et al, 1997; He et al, 1995; lyer et al, 1996; Trego et al, 2011). The patches 1-377 and 747-928 of XPG were also shown to interact with XPB and XPD. Monomeric XPG is uniformly distributed throughout the cell and translocates upon UV irradiation into the nucleus. Besides, XPG binds to NER complexes for approximately four minutes with a half-life of 200 seconds and UV-damage sites via its TFIIH interactions in vitro (Park et al, 1996; Zotter et al, 2006). A single mutation of an essential residue in the PIP-box of XPG (R992A/E) showed a slightly reduced NER activity in a luciferase 
reporter gene assay (Gary et al, 1997). During this thesis a mutant, where the two essential residues for PCNA interaction F996 and F997 were mutated to alanine, FF is used (see chapter 4.2.1).

Several splicevariants (isoforms) of the XPG gene were identified on mRNA- and protein expression level in samples of diverse human fibroblasts (wt) and other human cell types (Emmert et al, 2001). One truncated splicevariant, isoform VI $(725+11$ intronic aa), was used during the experiments of this thesis. The functionality of Isoform $\mathrm{VI}$ and its significance during NER will be amongst others investigated in this thesis (see chapter 4.2.1). The artificially created truncated Del variant (948 aa) was used as a control (Gary et al, 1997). Furthermore several double and triple mutants were created.

Very little is known about XPG's secondary modifications, but it is possible that its activity is at least in part modulated by phosphorylation like it was postulated in the past, because several potentially strong phosphorylation sites were identified in silico, e.g. serine 310 (http://www.phosphosite.org) (Savas \& Ozcelik, 2005). However, XPG is proposed to be phosphorylated by TFIIH as part of modulation of the later recruitment of downstream synthesis factors, but a clear proof remains missing (unpublished data in (Winkler et al, 2001)). Tillhon and colleagues reported acetylated XPG in a p300/CBP- dependent manner. However, XPG acetylation was inhibited by PCNA in a p21dependent manner and was associated with proper DNA repair synthesis (Tillhon et al, 2012).

\subsubsection{Structural and endonucleolytic function of XPG during NER and transcription}

The occurrence of XP/CS phenotype is thought to be developed in XPG patients (see chapter 1.1.2) as the consequence of an encoded C-terminally truncated protein and thus a destroyed TFIIH interaction (in particular the abolished interaction between the CAK- and the core complex of TFIIH, see Figure 7) and the subsequent failure during the transactivation of nuclear receptors (Ito et al, 2007; Scharer, 2008b). Contrary, the recent work from our lab reports three XP-G/CS patients expressing full-length XPG protein, which points towards the identification of essential residues involved in this process (Schafer et al, 2013b).

Beside its endonucleolytic activity during NER, XPG comprises several structural roles: the stabilization of TFIIH during formation of the transcription bubble, inducing the first cut by XPFERCC1, and protecting PCNA from interference by its inhibitor p21 (Evans et al, 1997; Ito et al, 2007; Mocquet et al, 2008; Staresincic et al, 2009) (see chapter 1.3.5). XPG was also shown to interact stably with transcription bubbles independently of a lesion, underlining its general role in basal, TFIIH associated, transcription (Sarker et al, 2005). 
Sarker and colleagues furthermore demonstrated in vitro, that the binding of XPG around the entire bubble is performed rather by the spacer than by the C-terminal region (PIP- box, D2, NLS-B,-C; see chapter 1.4.2) of XPG. Neither the spacer nor the C-terminal end of XPG (>1012 aa) are required for endonucleolytic activity but for stable bubble binding in an ATP-dependent manner, preferentially with bubble-Pol II complexes, rather than with bubbles alone. Thus, the ATPase activity of CSB (see chapter 1.3.4.1.2) induces a conformational change in Pol II, which physically and functionally separates both activities, the binding of XPG to the (lesion containing) DNA and its endonucleolytic activity, into two subsequent steps.

The spacer (aa86 - aa765) is not required for full TFIIH interaction, what narrows the interaction domains down, compared to lyer et al., to aa1-85 (see Figure 10) (lyer et al, 1996; Sarker et al, 2005). In this context the GST-tagged C-terminal domain of XPG alone was sufficient to stimulate CSB ATPase activity in presence of a transcription bubble (Sarker et al, 2005).

Another function of XPG in transcriptional context was reported by Le May and colleagues in the year 2012. They revealed, that DNA breaks and DNA demethylation events at promoters of activated genes are dependent on XPG's endonuclease activity, allowing recruitment of the CTCF chromatin organizer and stabilizing XPF at the terminator of the gene, leading to loop formation between these regions and control of gene expression (Le May et al, 2012). In yeast, the transcriptional mediator (subunit med17) was found to recruit Rad2 (XPG homolog of s. cerevisiae) to transcriptional sites in the absence of exogenous genotoxic stress (Eyboulet et al, 2013). Further a med17 mutant showed decreased occupancy of Rad2 at transcriptionally active genes (Pol II, III) after UV irradiation, demonstrating the tight connection between transcription and DNA repair. Noteworthy, Rad2 was also found to be involved in the regulation of the cell-cycle and actin dynamics in S. cerevisiae after UV irradiation (Kang et al, 2014). The fact that Rad2 was found to be transcriptionally active in an endonucleolytic independent manner supports the idea of a dual or multiple role for XPG and the implications for the XP/CS combined phenotype (see chapter 1.1.2) (Lee et al, 2002; Scharer, 2008b).

\subsubsection{Involvement of XPG in other (DNA repair) pathways}

Like XPC, CSA, and CSB are correlated with the removal of oxidative damage, XPG-CS patients have been found to be defective in, especially mitochondrial, Base excision Repair (BER) (Cooper et al, 1997; D'Errico et al, 2006; Dianov et al, 1999; Kamiuchi et al, 2002). The XPG protein enhances the in vitro repair of oxidative damage by recruiting NTH1 to damaged sites and stimulating its glycosylase activity in an endonuclease- independent fashion. This connects XPG to the base excision repair pathway and this potentially combines deficiencies in oxidative damage removal, in addition to 
transcriptional deficiencies, with the XP-G/CS phenotype (see chapter 1.1.2) to (Bessho, 1999; Klungland et al, 1999; Melis et al, 2013).

XPG was found to interact directly with the Werner syndrome (WS) WRN 3'-5' RecQ-like helicase, stimulating its activity and cooperatively annealing two single strands together during DNA replication or rather at stalled replication forks (Trego et al, 2011). WRN defective genes cause the Werner syndrome (WS), which is another progeroid disease as XP, associated with defects in Base excision repair, premature aging, chromosomal stability, and shows increased cancer development (Rossi et al, 2010).

WS- cells are sensitive to cisplatin, which causes DNA lesions repaired by the NER pathway (see chapter 1.3.2). WRN co-localizes with RPA (in a conserved fashion) in response to DNA damaging agents (Hyun et al, 2012; Sakamoto et al, 2001). Furthermore, WRN causing deficiency in Pollltranscription leads to speculations if this association is maybe more important in the cellular context and for XP/CS phenotype development than it has been assumed (Balajee et al, 1999; Harrigan et al, 2006). However, more elucidations are required.

XPG is not essential for, but able to stimulate hairpin removal of (CAG)n/(CTG)n DNA hairpins, normally repaired by the Hairpin repair pathway (HRP), by enhancing cleavage 5 ' of the structure, demonstrating the manifold structures cleaved by XPG (Hou et al, 2011; Zhang et al, 2012).

\subsection{Other Nucleases investigated during this study}

This thesis investigates a possible backup mechanism for the function of XPG in living cells. Some candidates for the fulfillment of this position are summarized below.

\subsubsection{Structurally XPG-related nucleases}

XPG is part in the highly conserved endonuclease Fen1- superfamily. Relevant family members for this thesis are presented.

\subsubsection{Flap endonuclease 1 (Fen1)}

Eukaryotic polymerases do not have a 5' nuclease activity like prokaryotic poll and use Fen1 (DNase IV) for cleavage of single stranded flaps during Okazaki fragment maturation (Burgers, 2009; Lyamichev et al, 1993). The structure specific flap endo-/exonuclease 1 (Fen1) is highly conserved and comprises distinct domains like the required $\mathrm{N}$ - and I- domain for nuclease function and a PIPbox for PCNA interaction as well as the C-terminal domain carrying two nuclear localization signals (see Figure 12, more detailed domain description see Figure 10) (Gary et al, 1997; Knauf et al, 1996; Lieber, 1997). Fen1 contains a spacer region, which is pretty short in comparison to XPG (70 amino 
acids, helical loop) and responsible for DNA-flap binding and thus activation of nuclease activity (Storici et al, 2002).

Fen1 is associated with several DNA repair pathways like Mismatch repair (MMR) and long patch Base excision repair (BER) (Lieber, 1997). In the context of BER, the NTH1 glycosylase (in XPG dependent manner, see chapter 1.4) is the damage sensor and creates an AP (apurinic) site for AP endonuclease 1 , which is able to cut the phosphate backbone and leave a $3^{\prime} \mathrm{OH}$ and $5^{\prime}$-deoxyribose phosphate (dRP) (Haukanes et al, 1990; Hazra et al, 2001). In most cases, Pol $\beta$ recruited to the AP site is able to excise the $5^{\prime}(\mathrm{dRP})$ and perform single nucleotide Repair (SN-BER) (Frosina et al, 1996). But sometimes the lyase activity of Pol $\beta$ is inhibited by the presence of an oxidized or reduced dRP, Pol $\beta$ performs a strand displacement, resulting in a longer flap (2-12 nts) and a substrate for Fen1 in the same fashion as during Okazaki fragment maturation (Klungland \& Lindahl, 1997; Prasad et al, 2000; Sattler et al, 2003). Unlike XPG (see chapter1.4), Fen1 is inhibited by folded flap structures like hairpins but nevertheless it is able to cut upstream of a hairpin, possibly occurring during end resection in trinucleotide repeat replication (Balakrishnan \& Bambara, 2013). Fen1 is involved in the recovery from UV-light-induced replication inhibition and is induced by p53 (Christmann et al, 2005). Fen1 also displays a Gap endonuclease (GEN) activity, which is connected to WRN, telomere stability and probably apoptosis (Liu et al, 2006; Saharia et al, 2008).

Its small protein size of $42 \mathrm{kDa}$ is sufficient to diffuse freely through nuclear pores (Qiu et al, 2001). However, to reach a practical rate of nuclear migration, Fen1 needs active Import into the nucleus. It was found in a complex with importin $\alpha$ in a c-terminal and NLS dependent manner and under UV in S-phase (de Barros et al, 2012).

A free $5^{\prime}$ single stranded flap capped with a TT CPD lesion was digested in in vitro nuclease assays in dependency of S. pombe Fen1 (Yoon et al, 1999). The model suggests the extrusion of the dsDNA by the action of the DNA polymerase, expanding the flap from 2nts to 9-11 nucleotides, thus making it a substrate for Fen1 digestion and subsequent nick sealing by a ligase. However, the NER reaction bubble displays a DNA lesion positioned exactly 9-11 nucleotides upstream of the downstream 3' branch (Tapias et al, 2004). During the intermediate state with the presence of a defective XPG, the putative action of DNA2 performing strand shortening, possibly until it drops off at the lesion, would lead to a structure which was shown to be cleaved by s. pombe Fen1. 


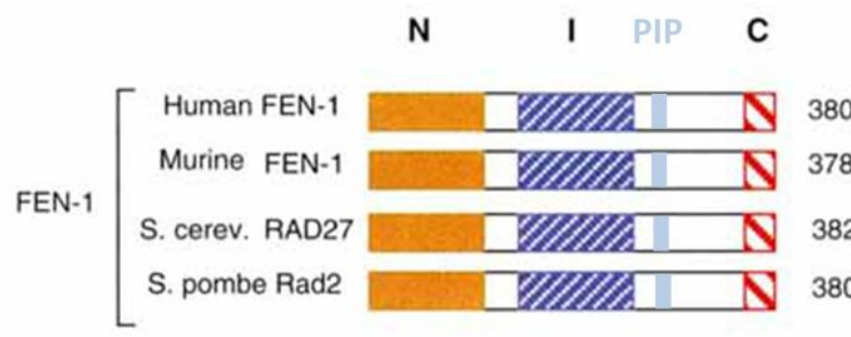

Bacteriophage
$5^{\prime}->3^{\prime}$
Exonucleases

T4 RNase $\mathrm{H}$

T5 Exo D15

T7 Gene 6 Exo

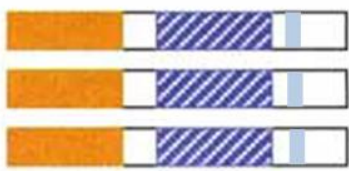

305

291

300
Figure 12: Fen1 superfamily domain conservation

The Fen-1 family nucleases comprise the highly conserved $\mathrm{N}$ - and I domains, bearing the nuclease function. The PCNA interaction is conserved, despite the PIP-box sequence is partially different. (adapted from Lieber, 1997)

(Cann \& Ishino, 1999; Matsumiya et al, 2002; Patoli et al, 2013)
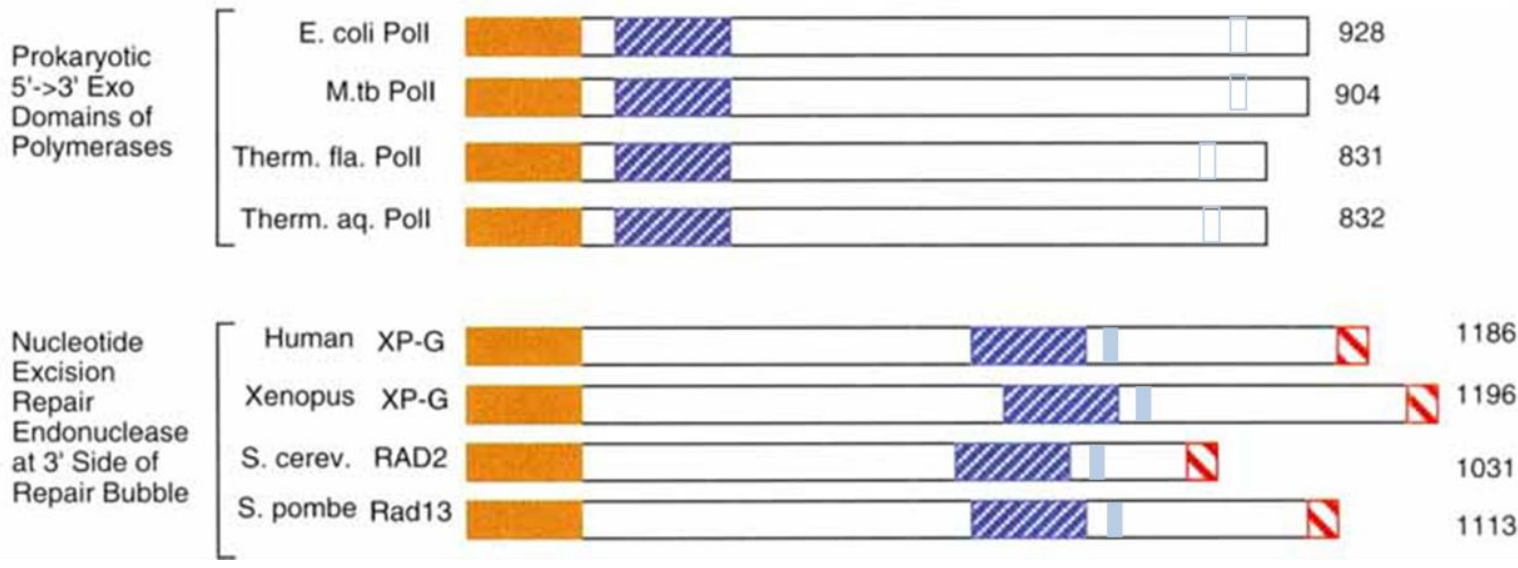

Fen1 is essential for Okazaki fragment maturation during replication and cuts the displaced RNA primer endonucleolytically (also see chapter 1.5.2) (Li \& Kelly, 1984). Among other structures (see Figure 13), ssDNA and RNA 5' flaps, which occur due to the action of processive polymerases, are digested by Fen-1 together with a little portion of the newly synthesized initiator DNA before Ligase I seals the nick.
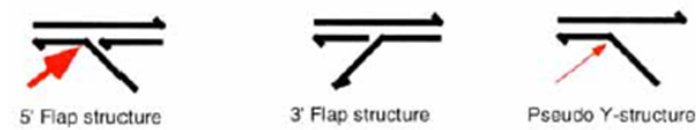

Psoudo Y-structure
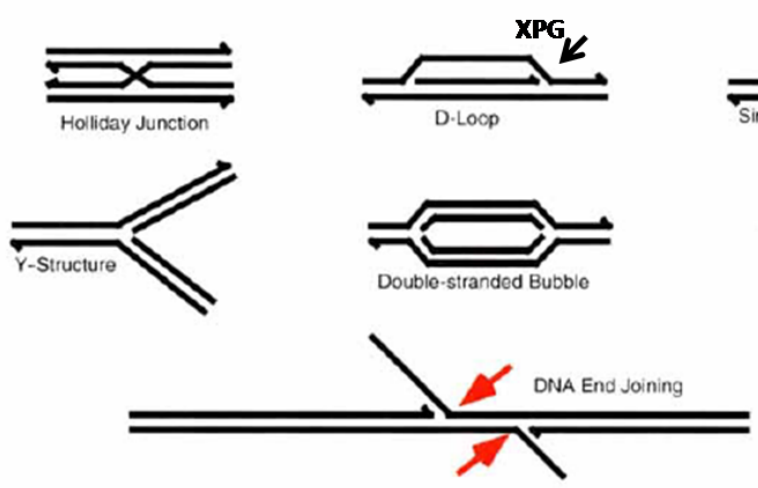
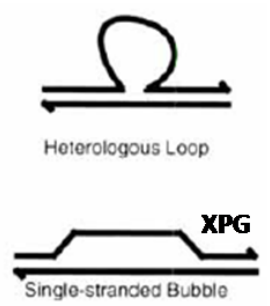

Heterologous Loop

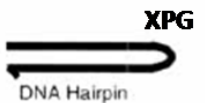

Figure 13: Structures cleaved by Fen 1

Red arrows = endonuclease activity of Fen1, thickness indicates strength of the substrate (Lieber, 1997). Additionally, structures demonstrateably cleaved by XPG are also illustrated (see chapter 1.4). 
The short flap degradation (long flaps see chapter 1.5.2) during replication most commonly occurs as nick translation (see Figure 14), the iterative cycle between Pol $\delta$ unwinding the fragment and Fen1 cutting the short (often 1nt) flap until the initiator RNA is degraded (Burgers, 2009). Fen-1 has about 30 interaction partners, among them PCNA (see chapter 1.3.5), DNA2 (see chapter 1.5.2), BLM, RPA (see chapter 1.3.4.2), and WRN (Balakrishnan \& Bambara, 2013). Fen1 is believed with a size of about $42 \mathrm{kDa}$ only to maximally bind two or three partners at once, raising the fact that there is a sequential hand off process between Fen1 and its several partners (Chapados et al, 2004; Prasad et al, 2011). However, the endonuclease activity of Fen 1 is increased up to 50 fold by binding one of its partners (Balakrishnan \& Bambara, 2013). Binding to the trimer PCNA displays the key interaction in this context. PCNA acts like a molecular switch, with each PCNA monomer bound to either Pol $\delta$, Fen1 and Ligase I (Dionne et al, 2003).

Also longer flaps do occur, which lead to hairpin formation (e.g. trinucleotide repeats; due to sequence context) or subsequent coating by RPA, which prevents Fen1 binding to the flap or its nuclease activity, respectively (Bae et al, 2001; Lieber, 1997; Rossi \& Bambara, 2006). Recruitment of a ligation system consisting of Fen1 and Ligase I is one possibility for the cell to terminate NER and close the nick after DNA resynthesis (Mocquet et al, 2008).

For complete catalytic activity, beside divalent metal ions, Fen1 needs a free 5' end to be stably recruited and loaded onto the ssDNA by threading the DNA through a hole in the molecule (Bae et al, 2001; Lieber, 1997; Rossi \& Bambara, 2006). This interaction bends the junction, also conferring structure specifity to Fen 1 and probably hinders other nucleases with similar substrates (ssDNAdsDNA junction: GEN1, Exo1, XPG) from wrong cleavage (Patel et al, 2012; Sobhy et al, 2013) (also see chapter 1.5.1.2). Furthermore, Fen1 needs a short 3' flap (1nt) for proper DNA binding and catalysis (Finger et al, 2009).

Secondary modifications of Fen1, same nuclease family like XPG (see chapter 1.4) occur in broad range incl. acetylation (by p300, e.g. in response to UV), on four specific lysine residues with an increasing rate of inhibition of Fen1's (substrate binding) activity proportional to the degree of acetylation (Hasan et al, 2001a; Hasan et al, 2001b). Acetylation also regulates RPA binding to ssDNA and strand displacement activity of Pol $\delta$ suggesting a defined acetylation based cellular mechanism to enhance safety/fidelity to the removal/repair of longer flaps during LP-BER (Balakrishnan \& Bambara, 2013).

Phosphorylation of Fen1 inhibits its nuclease activity and prevents PCNA from binding, and was further shown to regulate its nucleolar localization as well as the UV repair capacity (Guo et al, 2008; Henneke et al, 2003). The methylation of Fen1 prevents its phosphorylation but leaves the 
interaction with PCNA unaffected (Guo et al, 2010; Zheng et al, 2011). This raises speculations about methylated Fen1 at the arrival and phosphorylated Fen1 after cleavage during handover with Ligase I. The phosphorylation also triggers its ubiquitination and subsequent sumoylation followed by degradation, for instance in G2/M phase, whereby Fen1 activity logically peaks in S phase (Guo et al, 2012). This illustrates the additional layer of regulation of Fen1, one of the most extensive studied proteins in the Fen1 superfamily, and rises implications for other family members, e.g. XPG (see chapter 1.4).

The Fen1 superfamily (e.g. GEN1, Exo1, XPG) shares conserved sequence homologies regarding the DNA binding mechanism, a helical gateway consistent of a $\mathrm{K}+$ bridge, the $\mathrm{H} 2 \mathrm{TH}$ (helix-2turn-helix) motif, and a helical turn, which are able to recognize several structures in sequence independent manner (Grasby et al, 2012; Tsutakawa et al, 2011; Tsutakawa \& Tainer, 2012).

Fen1, as already mentioned above, interacts with more than 30 proteins and is implicated in several pathways like apoptosis and DNA repair, the resolution of stalled replication forks, the removal of hairpins, and telomere replication, designating the multiplayer Fen1 as a tumor suppressor (Zheng et al, 2011). However, the occurrence of ssDNA flaps with free $5^{\prime}$ ends if the second $3^{\prime}$ cut by XPG is missing. This structure was assumed to be target for Fen1 and it was speculated about a backup mechanism of NER by Fen1 before (Godon et al, 2012; Matsumoto, 2001). Moreover, a potential backup mechanism for XPG during NER was postulated before, in order to explain the Fen1 UV resistance capacity (Matsumoto, 2001). However, the Rad27-Rad2 mutant (Fen1-XPG homologues in yeast) displayed no synergistic negative effect regarding the cellular UV damage repair capacity (Qiu et al, 1998).

\subsubsection{Exonuclease 1 (Exo1)}

There is evidence for the recruitment of Exonuclease 1 (Exo1) to sites of local damage in presence of a huge overload of UV-derived DNA lesions or the inhibition of DNA repair synthesis by polymerase inhibitors, nucleoside analogues or an endonuclease defective XPG in yeast and human cells (Giannattasio et al, 2010; Overmeer et al, 2011; Scharer, 2013; Sertic et al, 2011). However, Exo1 activity promotes processive end resection over long stretches of dsDNA resulting in huge ssDNA gaps. This was shown to be covered by RPA and resulted in the activation of ATM/ATR DDR pathway in non-dividing cells (see chapter 1.3.1).

Exo1 is Fen 1 family member and shares features with the respective family members Fen 1 and XPG (see chapters 1.4, 1.5.1.1). Despite sequence homologies, especially concerning the nuclease domain and a proposed similar mechanism of DNA binding, Exo1 was similarly shown to interact with PCNA. 
Noteworthy, the Exo1-PCNA interaction confers processivity to the nuclease, like it was shown in vitro DSB repair assays (Chen et al, 2013; Constantinou et al, 1999; Grasby et al, 2012).

The human homologue of Exo1 displays the same exo- as well as endonuclease activities like human Fen1 (Lee \& Wilson, 1999). However, s. cerevisiae exo1 ${ }^{\text {- }}$ cells are not sensitive to UV and are able to perform normal NER. The possibility, that the respective homologues of Fen1 and Exo1 provide convertible back up pathways for both, replication and UV response, was assumed since overexpression of Exo1 in fission yeast is lethal after UV damage (McCready et al, 2000).

\subsubsection{DNA2}

Longer $5^{\prime}$ ssRNA/DNA RPA coated flaps ( $>25 \mathrm{nts}$ ) can occur during replication to a minor extend (Burgers, 2009). These are first shortened by the action of the helicase/nuclease DNA2 to short flaps (2-6nts), afterwards RPA is displaced, and leaves a substrate for Fen1 (see Figure 14).

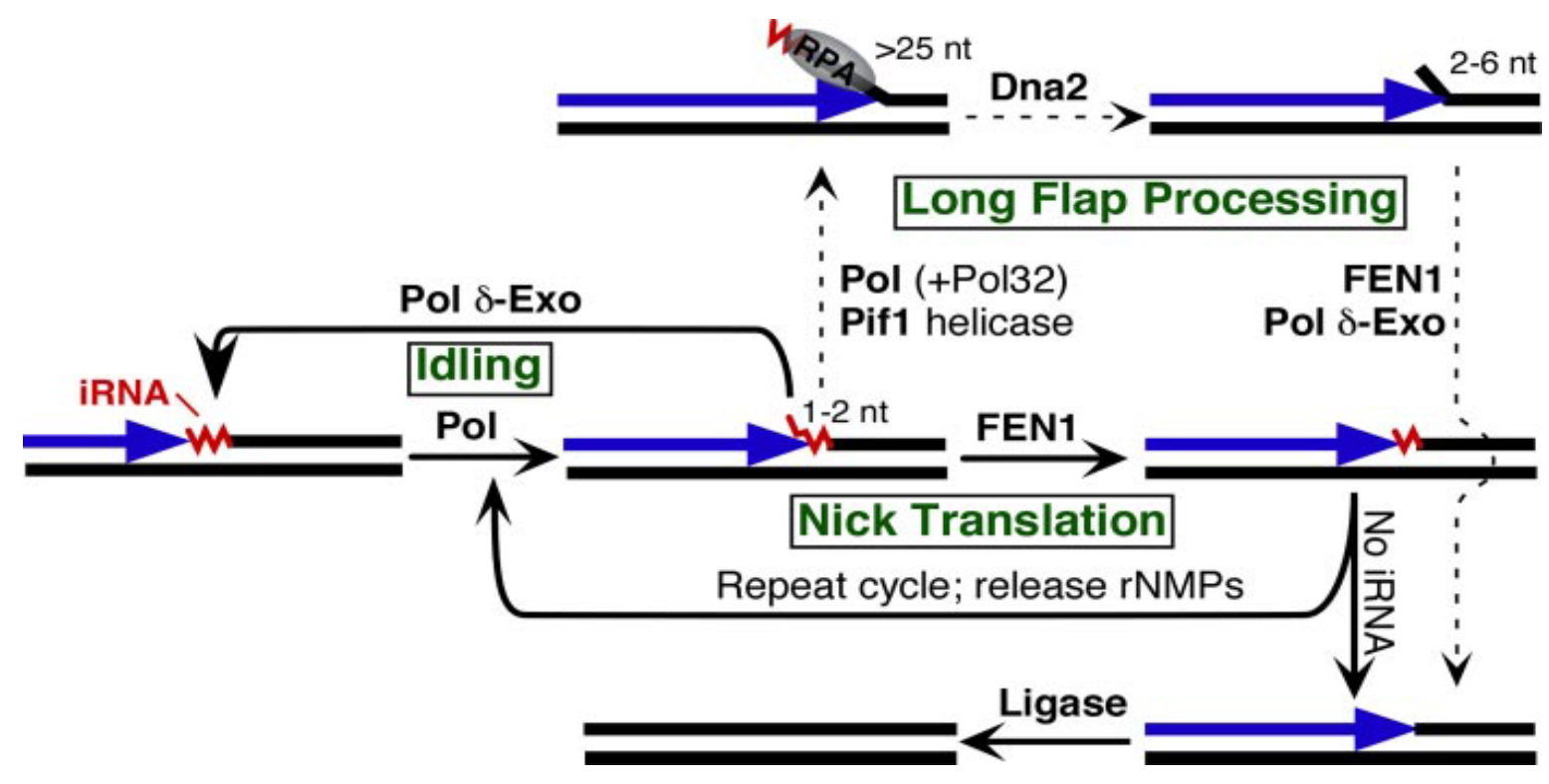

Figure 14: Okazaki Fragment maturation

Long flap processing requires DNA2 whereas Fen1 activity is sufficient to cleave short flaps during nick translation. At the end of both sub pathways the RNA primer is removed and a recruited ligase seals the nick (Burgers, 2009).

DNA2 has two key features, helicase and nuclease function, but rather its helicase function than its nuclease function is essential for replication (Budd \& Campbell, 2000). DNA2 acts on longer flaps ( 30nts), preferentially coated with RPA, the activator for DNA2, cutting it down to a short flap which is substrate for Fen1 (2-6nts) (Burgers, 2009). DNA2 is strongly stimulated (ATPase, helicase, and nuclease activity) by acetylation (Balakrishnan et al, 2010).

DNA2 and Fen1 work in a tight connection, DNA2 overexpression is able to compensate a defective Fen1 and vice versa, and a double mutant is lethal in s. cerevisiae (Budd \& Campbell, 2000). Like 
Fen1, DNA2 is also inhibited by the formation of hairpins (HRP). These structures are in turn is a target for helicase PIF1, probably leading to an unwinding of the whole Okazaki fragment and recruitment of Pol $\delta$ (Pike et al, 2009; Pike et al, 2010; Ryu et al, 2004).

\subsection{Aim of research}

The aim of this study is to further investigate the multifunctional endonuclease XPG and its biological and functional roles during Nucleotide excision repair (see chapter 1.3.2). The biochemical roles of XPG are very diverse due to its manifold interactions with several binding partners in cell nuclei (see chapter 1.4). The specification of the XPG interactions, with regard to its functional domains, and its influence on NER, is analyzed in this thesis on the supposition of the following three guiding objectives:

The first aim was to explore XPG deficient patients and expand the scientific knowledge about spectrum of physiologically occurring XPG mutations as a model for XPG protein functions (see chapter 1.1). In this context, the identification and characterization of the disease-causing mutation of a new XPG patient XP172MA is displayed. The genotype-phenotype correlation during this thesis narrows down the TFIIH interaction domain of the XPG N-terminus.

The second task was to examine if the functional XPG-PCNA interaction is responsible for PCNA recruitment during NER. I've created several mutants of XPG with specific PCNA- and ubiquitininteraction abolishing mutations (see chapters $1.3 .5,1.4 .2$, and 4.2.1). These mutants are analyzed with several functional assays to determine their NER activity (HCR), their influence on the immediate DNA repair synthesis (UDS) and, at least in part, their ability to recruit PCNA to local UVdamage sites (Triple-IF) (see chapters 3.5.1, 3.5.2, 3.5.4).

The third objective was to investigate a potentially alternative pathway during the very last steps of NER in dependency of the presence of a functionally constricted XPG, e.g. truncated or endonuclease defective. The DNA structure during the intermediate state of dual incision raises the possibility of DNA2 and/or Fen1 recruitment to sites of ongoing NER and is similarly investigated in this thesis (see Figure 8, see chapter 1.5). 


\section{Materials}

\subsection{Biological material}

\subsubsection{Bacterial strains}

Escherichia coli (E. coli) DH5 $\alpha$ [F-, endA1, glnV44, thi-1, recA1, relA1, gyrA96, deoR, nupG, Ф80dlacZAM15, $\Delta$ (lacZYA-argF)U169, hsdR17(rK- mK+), $\lambda-$,] (Hanahan, 1983) was used as host strain for plasmid DNA amplification.

\subsection{2 primary fibroblast cell culture}

Functional assays were performed using three different fibroblast cultures. WT41, WT-thilo and XP172MA derived from a skin punch biopsies of two healthy and a XP donor, respectively (see chapter 3.3.1). XP20BE (Moriwaki et al, 1996), a Xeroderma Pigmentosum (XP) group G fibroblast culture, was purchased at the Coriell cell repository (Cat. No. AG08803, Camden New Jersey, USA). HeLa cells (immortal, cervical cancer, donor: Henrietta Lacks) were purchased at the ATCC (Manassas Virginia, USA) (Scherer et al, 1953).

\subsection{Devices}

Table 1: Devices

\begin{tabular}{lll} 
Description & Label & Supplier \\
\hline \hline \multirow{2}{*}{ Agarose gel melting } & Microwave NN-5253 & $\begin{array}{l}\text { Panasonic, Hamburg, } \\
\text { GER }\end{array}$ \\
\hline Agarose gel trays and chambers & Blue Marine 200 & Serva, Heidelberg GER \\
\cline { 2 - 3 } & $\begin{array}{l}\text { Spezial Gelelektrophoresekammer } \\
\text { für Agarosegele }\end{array}$ & $\begin{array}{l}\text { Hardware \& service, } \\
\text { Friedland GER }\end{array}$ \\
\hline Aqua bidest. supplier & arium ${ }^{\circledR}$ 611 VF & Sartorius, Göttingen GER \\
\hline Autoclave & DE-65 & Systec, Wettenberg GER \\
\hline Bacterial culture & Erlenmeyer flask (200ml, 500ml) & VWR, Darmstadt GER \\
\hline Centrifuges & Mikro 220 & Hettich, Tuttlingen GER \\
\cline { 2 - 3 } & Benchtop centrifuge 5415, 1-15 & Sigma, Munich GER \\
\cline { 2 - 3 } & Sorvall RC 6+ & Thermo Scientific, \\
& Wega fuge 1,0R, model G25 & $\begin{array}{l}\text { Thermo Scientific, } \\
\text { Centrifuges }\end{array}$ \\
\cline { 2 - 3 } & MC 6 centrifuge & Sarstedt, Nümbrecht \\
& GER \\
\hline Counting chamber & Capsule HF-120 & Tomy Seiko JPN \\
\hline Dish washer & Neubauer cell counting chamber & Brand, Wertheim GER \\
\hline
\end{tabular}




\begin{tabular}{|c|c|c|}
\hline Dispenser & Multipette ${ }^{\circledR}$ plus & $\begin{array}{l}\text { Eppendorf, Hamburg, } \\
\text { GER }\end{array}$ \\
\hline \multirow{2}{*}{ Drier } & \multirow{2}{*}{ SL 50} & Memmert, Schwabach \\
\hline & & GER \\
\hline Freezing & Cryo $1^{\circ} \mathrm{C}$ cooler & VWR, Darmstadt GER \\
\hline \multirow[t]{4}{*}{ fridges and freezers } & GUS 34420 & Bosch, Stuttgart GER \\
\hline & $\operatorname{Arctis}\left(-20^{\circ} \mathrm{C}\right)$ & AEG, Nürnberg GER \\
\hline & Ultra low MDF-473V $\left(-80^{\circ} \mathrm{C}\right)$ & Sanyo, Munich GER \\
\hline & glassline $\left(+4^{\circ} \mathrm{C}\right)$ & $\begin{array}{l}\text { Liebherr, Ochsenhausen } \\
\text { GER }\end{array}$ \\
\hline \multirow[t]{4}{*}{ Gel documentation system } & Biodoc controller + CCD-camera & $\begin{array}{l}\text { Biometra, Göttingen, } \\
\text { GER }\end{array}$ \\
\hline & UV-table Fluo-Link & $\begin{array}{l}\text { Biometra, Göttingen, } \\
\text { GER }\end{array}$ \\
\hline & video graphic printer UP-890CE & Sony, Berlin GER \\
\hline & video monitor wv-BM 900 & $\begin{array}{l}\text { Panasonic, Hamburg } \\
\text { GER }\end{array}$ \\
\hline \multirow[t]{2}{*}{ Glasspipettes } & Blau Brand $(5,10,20,25 \mathrm{ml})$ & Brand, Wertheim GER \\
\hline & Hirschmann EM $(5,10,20,25 \mathrm{ml})$ & $\begin{array}{l}\text { Hirschmann, Eberstadt } \\
\text { GER }\end{array}$ \\
\hline Ice machine & ZBE 30-10 & Ziegra, Isernhagen GER \\
\hline Imaging devices & LAS 4000 & Fujifilm, Düsseldorf GER \\
\hline \multirow[t]{3}{*}{ Incubators } & $\mathrm{CO}_{2}$-Incubator, MCO-18AIC & Sanyo, Munich, GER \\
\hline & Incubator model 200 & $\begin{array}{l}\text { Memmer, Büchenbach, } \\
\text { GER }\end{array}$ \\
\hline & Shaker Thermotron & Infors, Bottmingen SWZ \\
\hline Laboratory hood & not specified & $\begin{array}{l}\text { Norddeutsche Laborbau, } \\
\text { Quickborn GER }\end{array}$ \\
\hline \multirow[t]{2}{*}{ Liquid nitrogen container } & Chronos Biosafe & $\begin{array}{l}\text { Messer Griesheim, Bad } \\
\text { Soden GER }\end{array}$ \\
\hline & Apollo Biosafe & $\begin{array}{l}\text { Messer Griesheim, Bad } \\
\text { Soden GER }\end{array}$ \\
\hline Luminescence measurements & $\begin{array}{l}\text { Glomax }{ }^{\mathrm{TM}} 96 \text { Microplate } \\
\text { Luminometer }\end{array}$ & $\begin{array}{l}\text { Promega, Mannheim } \\
\text { GER }\end{array}$ \\
\hline \multirow[t]{3}{*}{ Microscopes } & $\begin{array}{l}\text { Axiovert } 100 \text { fluorescence } \\
\text { microscope }\end{array}$ & $\begin{array}{l}\text { Carl Zeiss, Oberkochen } \\
\text { GER }\end{array}$ \\
\hline & Axiovert Imager.M1 & $\begin{array}{l}\text { Carl Zeiss, Oberkochen } \\
\text { GER }\end{array}$ \\
\hline & BZ-9000 & $\begin{array}{l}\text { Keyence, Neu-Isenburg } \\
\text { GER }\end{array}$ \\
\hline Mobile pipettor & Pipetboy acu & $\begin{array}{l}\text { Integra Biosciences, } \\
\text { Fernwald, GER }\end{array}$ \\
\hline pH-Meter & digital pH-meter, pH 525 & Schütt, Göttingen GER \\
\hline Pipettes & $\begin{array}{l}\text { Transferpette }^{\circledR} \mathrm{S}(1000, \\
100,20,10 \mu \mathrm{l})\end{array}$ & Brand, Wertheim GER \\
\hline Post UV survival measurment & Elisa Reader & Tecan, Männedorf GER \\
\hline Power supply & Mini Power Rack P20 & $\begin{array}{l}\text { Biometra, Göttingen, } \\
\text { GER }\end{array}$ \\
\hline
\end{tabular}




\begin{tabular}{|c|c|c|}
\hline Protein concentration & DU 640® Spectrophotometer & Beckmann, Munich GER \\
\hline \multirow[t]{2}{*}{ Scales } & MC1 AC210S & Sartorius, Göttingen GER \\
\hline & Analytic balance BP2100 & Sartorius, Göttingen GER \\
\hline \multirow[t]{2}{*}{ SDS gel chamber } & Mini-PROTEAN Tetra Cell & Biorad, Munich GER \\
\hline & Hoefer SE600 Ruby & GE Heathcare \\
\hline \multirow[t]{2}{*}{ Spectrophotometer } & DU 640® Spectrophotometer & Beckmann, Munich GER \\
\hline & NanoVuePlus & $\begin{array}{l}\text { GE Healthcare, } \\
\text { Buckinghamshire UK }\end{array}$ \\
\hline Sterile bench & Technoflow 2F180-11 GS & $\begin{array}{l}\text { Integra Bioscience, } \\
\text { Fernwald GER }\end{array}$ \\
\hline Tamping machine & Tecnomara, Tecnoplug & $\begin{array}{l}\text { Integra Bioscience, } \\
\text { Fernwald, GER }\end{array}$ \\
\hline \multirow[t]{2}{*}{ Thermal Cycler } & Arktik Thermal Cycler & $\begin{array}{l}\text { Thermo Scientific, } \\
\text { Waltham USA }\end{array}$ \\
\hline & T Gradient Thermo Block & $\begin{array}{l}\text { Biometra, Göttingen, } \\
\text { GER }\end{array}$ \\
\hline Thermoblocks & Thermomixer 5436 & $\begin{array}{l}\text { Eppendorf, Hamburg, } \\
\text { GER }\end{array}$ \\
\hline UV-irradiation & UVC 500 crosslinker & $\begin{array}{l}\text { Amersham Biosciences, } \\
\text { Glattburg CH }\end{array}$ \\
\hline Vortexer & Vibrofix VF1 Electronic & $\begin{array}{l}\text { IKA Labortechnik, } \\
\text { Staufem, GER }\end{array}$ \\
\hline Water bath & 1003 & $\begin{array}{l}\text { GFL, Großburgwedel } \\
\text { GER }\end{array}$ \\
\hline Western Blot development & LAS 4000 & Fujifilm, Düsseldorf GER \\
\hline \multirow[t]{2}{*}{ Western blot equipment } & Mini Rocking Platform & $\begin{array}{l}\text { Biometra, Göttingen } \\
\text { GER }\end{array}$ \\
\hline & $\begin{array}{l}\text { Trans-Blot }{ }^{\circledR} \text { TurboTM Transfer } \\
\text { System }\end{array}$ & Biorad, Munich GER \\
\hline
\end{tabular}

\subsection{Disposables}

Table 2: Disposables

Label

96 well GlomaxTM 96 Microplate

Blotting paper extra thick

Cell culture flasks $(25 \mathrm{~cm} 3,75 \mathrm{~cm} 3,175 \mathrm{~cm} 3)$

Cell scraper $25 \mathrm{~cm}$

Combitips advanced ( $5 \mathrm{ml})$

Conical tubes (Falcon $15 \mathrm{ml} \& 50 \mathrm{ml}$ )

cryo boxes

Cryo tubes $2 \mathrm{ml}$

Glass cover slips, optical planed

\section{Supplier}

Promega, Mannheim GER

Biorad, Munich GER

Greiner bio-one, Frickenhausen

GER

BD Biosciences, Pharmingen, Oxford UK

Eppendorf, Hamburg GER

Sarstedt, Nümbrecht GER

Nunc, Wiesbaden GER

Greiner bio-one, Frickenhausen

GER

Thermo Fisher, Karlsruhe GER 
Glass cover slips, round, $20 \mathrm{~mm}$

Isopore membrane filters $5 \mu \mathrm{m}$

Kimtech wipes

Mini TGX Gels

Multiply $\mu$ Strip Pro 8-strip

Nitrocellulose, $0.45 \mu \mathrm{M}$ Protran BA85

Object slide

Parafilm ${ }^{\circledast}$

Petri dishes

Pipette tips $(10 \mu \mathrm{l}, 200 \mu \mathrm{l} \& 1000 \mu \mathrm{l})$

Polystyrene round bottom tube

Reaction tubes $(0,5 \mathrm{ml}, 1,5 \mathrm{ml} \& 2 \mathrm{ml})$

Scalpel, disposable

Syringes, single use $(2 \mathrm{ml}, 5 \mathrm{ml}, 10 \mathrm{ml} \& 20 \mathrm{ml})$

Sterile filters (pore size $0.2 \mu \mathrm{m} \& 0.45 \mu \mathrm{m}$ ) filtropur $\mathrm{S}$ (plus)

Tissue culture plates (24-, 96-well)

Toothpick

Wadding

Weighing paper (round/folded, 125mm)
Roth, Karlsruhe GER

merck Millipore, Darmstadt GER

Biorad, Munich GER

Sarstedt, Nümbrecht GER

Whatman, Madstone UK

Thermo Scientific,

Braunschweig GER

Pechiney Plastic Packaging

Greiner bio-one, Frickenhausen

GER

Sarstedt, Nümbrecht GER

BD Falcon, Heidelberg GER

Eppendorf, Hamburg GER

Feather, Osaka JPN

Braun, Melsungen GER

Sarstedt, Nümbrecht GER

Greiner

Fackelmann, Hersbruck GER

Brand, Wertheim GER

Schleicher \& Schüll, Dassel GER

\subsection{Chemicals, media and reagents}

Table 3: Chemicals, media and reagents

\begin{tabular}{|c|c|}
\hline Chemical/Reagent & Supplier \\
\hline 2-propanol & JT Baker, Deventer NED \\
\hline $6 x$ loading dye & $\begin{array}{l}\text { Fermentas, St. Leon-Rot, } \\
\text { GER }\end{array}$ \\
\hline Agarose Saekem $^{\circledR}$ & $\begin{array}{l}\text { Fluka Chemie, Neu-Ulm, } \\
\text { GER }\end{array}$ \\
\hline Alexa Fluor ${ }^{\circledast} 488$ Azide & $\begin{array}{l}\text { Life technologies, } \\
\text { Darmstadt GER }\end{array}$ \\
\hline Ammonium chloride $\left(\mathrm{NH}_{4} \mathrm{Cl}\right)$ & Merck, Darmstadt GER \\
\hline Ammonium persulphate (APS) & Sigma-Aldrich, Munich GER \\
\hline Ampicillin & Sigma-Aldrich, Munich GER \\
\hline Aqua ad iniectabilia & Braun, Melsungen GER \\
\hline Attractene Transfection Reagent & Qiagen, Hilden GER \\
\hline Boric acid & Merck, Darmstadt GER \\
\hline Bovine serum albumine (BSA) & PAA, Coelbe GER \\
\hline Bradford Mix Roti ${ }^{\circledR}$ & Quant Roth, Karlsruhe GER \\
\hline Bromphenol Blue & Sigma-Aldrich, Munich GER \\
\hline BSA (bovine serum albumin) & NEB, Ipswich USA \\
\hline Calcium chloride $\left(\mathrm{CaCl}_{2}\right)$ & Merck, Darmstadt GER \\
\hline Complete ULTRA Tablets Mini EDTA & Roche, Mannheim GER \\
\hline Dimethylsulfoxide (DMSO) & Sigma-Aldrich, Munich GER \\
\hline
\end{tabular}


Dithiothreitol (DTT)

dNTPs

Dulbecco's modified Eagle's medium (DMEM)

Ethanol (analytical grade)

Ethidium bromide (1\%)

Ethylenediaminetetraacetic acid (EDTA)

Fetal Bovine Serum (FBS)

Gene Ruler (100bp \#SM0241, 1kb \#SM0311) DNA Ladder

Glycerol

Glycine (C2H5NO2)

$\mathrm{HCl}$

Hi-Di Formamide

Isopropanol $\left(\mathrm{C}_{3} \mathrm{H}_{7} \mathrm{OH}\right)$

Kalium hydrogen phosphate $\left(\mathrm{KH}_{2} \mathrm{PO}_{4}\right)$

kanamycin sulfate

LB Agar

LB Broth Base (Bertani, 1951)

Magnesium chloride $\left(\mathrm{MgCl}_{2}\right)$

Marker VI,protein ladder

Methanol $\left(\mathrm{CH}_{3} \mathrm{OH}\right)$

$\mathrm{N}, \mathrm{N}, \mathrm{N}, \mathrm{N}$-tetramethyl-ethane-1,2-diamine (TEMED)

Nail polish (Last Forever)

Nonidet P40

Paraformaldehyde (PFA)

Penicillin-Streptomycin (100x)

Phenylmethanesulfonylfluoride (PMSF)

Ponceau S

Potassium Chloride $(\mathrm{KCl})$

Protease Inhibitor Cocktail (P2714)

Sodium acetate

Sodium Chloride ( $\mathrm{NaCl}$ )

Sodium dodecyl sulfate (SDS)

Sodium hydrogen phosphate $\left(\mathrm{Na}_{2} \mathrm{HPO}_{4} \times 2 \mathrm{H}_{2} \mathrm{O}\right)$

Sodium hydroxide $(\mathrm{NaOH})$

Succrose/ Saccharose

Trifluoroacetic acid (TFA)

Tris-Base

Triton X-100

Trypanblue

Trypsin/EDTA
Sigma-Aldrich, Munich GER

Fermentas, St. Leon-Rot,

GER

PAA, Coelbe GER and others

Merck, Darmstadt GER

Roth, Karlsruhe, GER

Sigma-Aldrich, Munich GER

Biochrom AG, Berlin GER

Fermentas, St. Leon-Rot,

GER

Merck, Darmstadt GER

Sigma-Aldrich, Munich GER

Merck, Darmstadt GER

Applied Biosystems, Foster

City, USA

Merck, Darmstadt GER

Merck, Darmstadt GER

Roth, Karlsruhe GER

Life technologies,

Darmstadt GER

Life technologies,

Darmstadt GER

Merck, Darmstadt GER

Applichem,Chicago Illinois,

USA

Mallinckrodt Baker,

Griesheim GER

P2 Kosmetik GmbH, Wr.

Neudorf GER

Sigma-Aldrich, Munich GER

Merck, Darmstadt GER

PAA, Coelbe GER

Sigma-Aldrich, Munich GER

Sigma-Aldrich, Munich GER

Merck, Darmstadt GER

Sigma

Sigma-Aldrich, Munich GER

Merck, Darmstadt GER

Roth, Karlsruhe GER

Merck, Darmstadt GER

Merck, Darmstadt GER

Sigma-Aldrich, Munich GER

Sigma-Aldrich, Munich GER

Merck, Darmstadt GER

Merck, Darmstadt GER

Sigma-Aldrich, Munich GER

Biochrom AG, Berlin GER 
Tween20

Vectashield Mounting Medium for Fluorescence H-1000

Vectashield Mounting Medium for Fluorescence with DAPI $\mathrm{H}-1200$

$\beta$-mercaptoethanol $\left(\mathrm{C}_{2} \mathrm{H}_{6} \mathrm{OS}\right)$
Merck, Darmstadt GER

Vector Laboratories, Burlingham CA/USA

Vector Laboratories, Burlingham CA/USA Merck, Darmstadt GER

\subsection{Antibodies}

Primary and secondary antibodies were used according to manufacturer's instructions and concentrations are listed in the respective method sections. PBS supplemented with $1 \%$ FBS was used as diluent.

Table 4: Primary antibodies

\begin{tabular}{lllll} 
Label & Dilution & Host & Isotype/clone & Supplier \\
\hline \hline CPD & $1: 1000$ & mouse & TDM-2, IgG2a k & $\begin{array}{l}\text { Cosmo Bio co., LTD, Tokyo JPN } \\
\text { (Mori et al, 1991) }\end{array}$ \\
PCNA & $1: 50$ & rabbit & FL-261 & Santa Cruz, Dallas USA \\
XPG & $1: 500$ & rabbit & A301-484A, IgG & Bethyl, Montgomery USA \\
B-Actin & $1: 5000$ & mouse & $\begin{array}{l}\text { anti human } \beta \text {-actin } \\
\text { clone AC-74 }\end{array}$ & Sigma-Aldrich, Munich GER
\end{tabular}

Dilutions were carried out in 5\% FBS in PBS or blocking solution (Western Blot).

Table 5: Secondary antibodies

\begin{tabular}{|c|c|c|c|c|}
\hline Label & Dilution & Host & Isotype/clone & Supplier \\
\hline $\begin{array}{l}\text { Alexa Flour }{ }^{\circledR} 594-\text { conjugated, } \\
\text { anti-mouse (RED) }\end{array}$ & $1: 500$ & goat & AffiniPure $F\left(a b^{\prime}\right)_{2}$ & Dianova, Hamburg GER \\
\hline Marina Blue anti rabbit (DAPI) & $1: 500$ & goat & $\lg G$ & $\begin{array}{l}\text { Life technologies, } \\
\text { Darmstadt GER }\end{array}$ \\
\hline $\begin{array}{l}\text { Alexa Flour }{ }^{\circledR} 405 \text {-conjugated } \\
\text { anti rabbit (DAPI) }\end{array}$ & $1: 500$ & goat & $\lg G$ & $\begin{array}{l}\text { Life technologies, } \\
\text { Darmstadt GER }\end{array}$ \\
\hline
\end{tabular}

\subsection{Enzymes}

Table 6: Enzymes

\begin{tabular}{ll} 
Label & Supplier \\
\hline \hline CIAP (calf intestinal alkaline phosphatase) $1 \mathrm{u} / \mu \mathrm{l}$ & MBI Fermentas, St. Leon-Rot GER \\
DNasel & Promega, Mannheim GER \\
Dpnl $10 \mathrm{u} / \mu \mathrm{l}$ & MBI Fermentas, St. Leon-Rot GER \\
Kpnl $10 \mathrm{u} / \mu \mathrm{l}$ & NEB, Ipswich USA
\end{tabular}


Pfu DNA Polymerase

Phusion DNA Polymerase

RNase A

T4 DNA Ligase $1 \mathrm{u} / \mu \mathrm{l}$

Taq DNA Polymerase

Xbal $10 \mathrm{u} / \mu \mathrm{l}$
MBI Fermentas, St. Leon-Rot GER

Finnzymes, Thermo Fisher,

Waltham USA

Sigma-Aldrich, Munich GER

MBI Fermentas, St. Leon-Rot GER

MBI Fermentas, St. Leon-Rot GER

NEB, Ipswich USA

\subsection{Buffers and solutions}

Unless otherwise noted all buffers and solutions are prepared in autoclaved aqua bidest. For sterile filtration $0,2 \mu \mathrm{m}$ filters and a syringe with an appropriate volume were used.

Table 7: Buffers and solutions

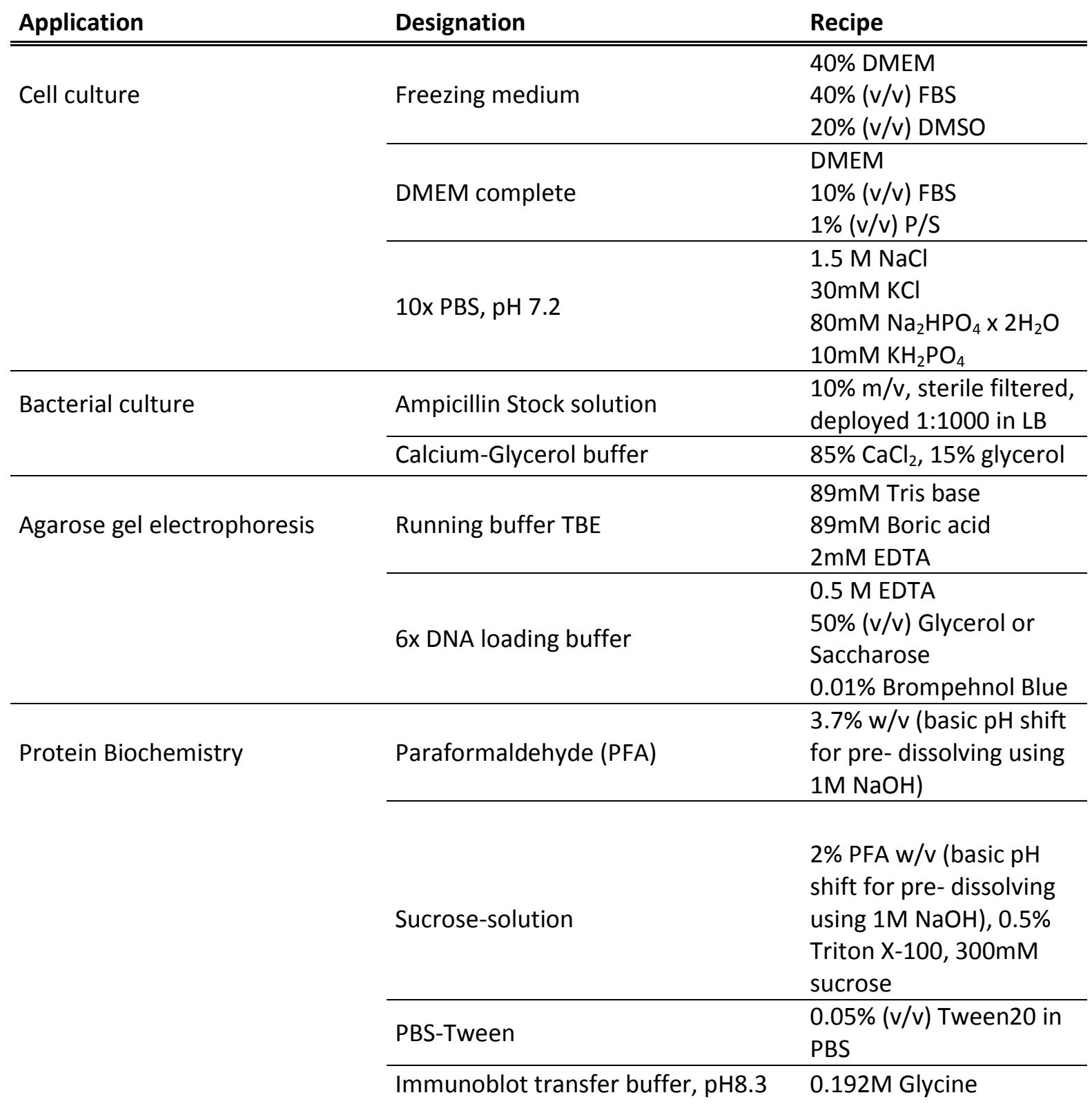




\begin{tabular}{ll}
\hline & $0.025 \mathrm{M}$ Tris-Base \\
& $20 \% \mathrm{MeOH}(\mathrm{v} / \mathrm{v})$ \\
& $0.01 \% \mathrm{SDS}$ \\
& $0.2 \%(\mathrm{v} / \mathrm{v})$ Ponceau S \\
Ponceau S-solution & $3 \%(\mathrm{v} / \mathrm{v})$ TFA \\
\hline \multirow{3}{*}{ SDS running buffer, $\mathrm{pH} 8.3$} & $0.192 \mathrm{M}$ Glycine \\
& $0.025 \mathrm{M}$ Tris-Base \\
& $0.1 \%$ SDS \\
\hline & $30 \mathrm{mM}$ Tris \\
& $3 \%$ SDS \\
SDS Page sample buffer, $\mathrm{pH} 4,7$ & $15 \%$ Glycerine \\
& $0,04 \%$ Brompehnol Blue \\
& before use add $10 \% \beta-$ \\
& mercaptoethanol \\
\hline
\end{tabular}

\subsection{Deoxyribonucleotides}

All oligonucleotides were synthesized by Sigma-Aldrich Co. LLC. Restriction site sequences were introduced at the $5^{\prime}$ end of the complementary primer annealing sequence extended upstream by a codon for proper enzyme binding (marked italic). Oligonucleotides used for quick-change mutagenesis included maximally two adjacent altered nucleotides flanked by 10 to 15 complementary bases, forward and reverse primers were placed in the same sequence context. Sequencing primers were designed for $55^{\circ} \mathrm{C}$ annealing temperature (see chapter 3.2.3.2)

Table 8: Primers for quick change mutagenesis

\begin{tabular}{ll} 
Description & Sequence 5'-3' \\
\hline \hline E791A_for & CCCATGGAAGCAGCCGCGCAGTGCGCC \\
E791A_rev & $\begin{array}{l}\text { GGCGCACTGCGCGGCTGCTTCCATGGG } \\
\text { (Constantinou et al, 1999) }\end{array}$ \\
\hline QC_D77A-for & CGTCCTATTTTTGTGTTTGCTGGGGATGCTCCAC \\
QC_D77A-rev & GTGGAGCATCCCCAGCAAACACAAAAATAGGACG \\
\hline QCXPG_PIP-N_1_for & GCATTACAGGAAGAGGCCTTTCATAATCCTCA \\
QCXPG_PIP-N_1_rev & TGAGGATTATGAAAGGCCTCTTCCTGTAATGC \\
\hline UBM_QC_1a_for & GAGGACTTCAGCAGCGCGCCCCCTGAAGTAAA \\
UBM_QC_1a_rev & TTTACTTCAGGGGGCGCGCTGCTGAAGTCCTC \\
UBM_QC_2a_for & GACTTCAGCAGCGCGGCCCCTGAAGTAAAGC \\
UBM_QC_2a_rev & GCTTTACTTCAGGGGCCGCGCTGCTGAAGTC
\end{tabular}

Table 9: Primers for cloning

\begin{tabular}{ll} 
Description & Sequence 5'-3' \\
\hline \hline DNA2-for_BamHI & TAG GGATCC-CTGTCTACAGTTTGCGATCC \\
DNA2-rev_Xhol & TAG CTCGAG - CCTAGTATGAAAAGGCAAGGG
\end{tabular}




$\begin{array}{ll}\text { JL_5-UTR-Kpnl-cl-f } & \text { TTAGGTACC-GTGCAGTCCGTCGTAGAAG } \\ \text { PCNA -Del2_Rev } & \text { TAGTCTAGA-CGATCCCTTCGAGTCATCCAC } \\ \text { PCNA-cl_II_for_Kpnl } & \text { TAGGAATTC-CGGCTATTCTCGCAGGATCAG } \\ \text { PCNA-Cl_II_rev_Xbal } & \text { TAGTCTAGA-TGCGACAAATTCATTACAAATGGC }\end{array}$

Table 10: Sequencing primers

\begin{tabular}{ll} 
Description & Sequence 5'-3' \\
\hline \hline DNA2_1100_for & GCCTGCCAACCATCTAGA \\
DNA2_1700_for & CCAGAATCAACTTTGTTCAG \\
DNA2_2300_for & CCATCCAATATTTTCCCGT \\
DNA2_2800_for & GTAACAGAAGCCAAACTCA \\
DNA2_500_for & AGCTCTGATCCAGCCACA \\
M13_rev & CAGGAAACAGCTATGAC \\
pCDNA3.1_rev & TAGAAGGCACAGTCGAGG \\
T7 & TAATACGACTCACTATAGGG \\
XPG_2472-2492 & CGGATCGCTGCTACTGTCACC \\
XPG_302-323 & AAACCAAGCACTTAAAGGAGTC \\
XPG_594r4 & TGCTGCTGTAGACGAAGGC \\
XPG_594r86 & CCTGTCAGTTTGCAAGAAACA \\
XPG_966-985 & CAACATTCAGGACACATCCG \\
XPG_EX8_for_SS & CTCGGAAGAAAGTGAATCTG \\
XPG_EX8_rev_SS & CACTTCTGCAACTGTCTTAGC \\
XPG_g2397for & ACAACTCTGAGAGGGACGAC \\
XPG_g285rev & CCCCATCAAACACAAAAATAGG \\
XPG_T10 & ACCTCTATGTTTTGCCTCCTT \\
XPG_xp5100 & TAAGACCTAATCCTCATGACA
\end{tabular}

\subsection{Plasmids}

Table 11: Plasmids

\begin{tabular}{lll} 
Label & Supplier & Application \\
\hline \hline 172MA_pcDNA3.1(+) (XPG patient) & (Lehmann, 2013) & Functional assays \\
IsoVI_pcDNA3.1(+) (XPG isoform) & (Lehmann, 2013) & Functional assays \\
pBactin-PCNA-Del & Dr. Ron Gary, UNLV (Gary et al, 1997) & subcloning \\
pBactin-PCNA-FF & Dr. Ron Gary, UNLV (Gary et al, 1997) & subcloning \\
pcDNA3.1(+) & Life technologies, Darmstadt GER, & cloning \\
pcmvLuc & Promega, Mannheim GER & HCR \\
pResQ shFEN3 3XF-FEN1 D181A & Addgene plasmid 17753, Sheila Stewart & subcloning \\
pResQ shFEN3 3XF-FEN1 wt & Addgene plasmid 17752, Sheila Stewart & subcloning \\
pRL-CMV & Promega, Mannheim GER & HCR
\end{tabular}




\subsection{Reaction systems}

Table 12: Reaction systems

\begin{tabular}{|c|c|}
\hline Label & Supplier \\
\hline BigDye Terminator v3.1 Cycle Sequencing Kit & Applied Biosystems, Foster City USA \\
\hline CellTiter 96® Non-Radioactive Cell Proiferation & Promega, Mannheim GER \\
\hline Click-iT EdU Alexa Fluor 488 Imaging Kit & Life technologies, Darmstadt GER \\
\hline Click-iT ${ }^{\circledR}$ Cell Reaction Buffer Kit & Life technologies, Darmstadt GER \\
\hline Dual-Luciferase Reporter Assay System & Promega, Mannheim GER \\
\hline NucleoBond® Xtra MiDi/Maxi & Machery + Nagel, Düren GER \\
\hline NucleoSpin ${ }^{\circledR}$ Gel extraction and PCR Clean Up & Machery + Nagel, Düren GER \\
\hline NucleoSpin $®$ Plasmid & Machery + Nagel, Düren GER \\
\hline QIAamp DNA Blood Kit & Qiagen, Hilden GER \\
\hline RevertAid H Minus First Strand cDNA synthesis Kit & MBI Fermentas, St. Leon-Rot GER \\
\hline RNase free DNase Set & Qiagen, Hilden GER \\
\hline RNeasy Mini Kit & Qiagen, Hilden GER \\
\hline $\begin{array}{l}\text { WesternBreeze Chemilumonescent Immunodetection } \\
\text { Systems anti mouse/rabbit }\end{array}$ & Applied Biosystems, Foster City USA \\
\hline
\end{tabular}

\subsection{Software, online tools and databases}

Table 13: Software, online tools and databases

\begin{tabular}{lll} 
Label & Supplier/web adress & Application \\
\hline \hline BZ-II Analyzer & Keyence & Triple-IF \\
BZ-II Viewer & Carl Zeiss, Jena GER & Fluorescence microscope \\
Axiovert Axiovision 100 & http://blast.ncbi.nlm.nih.gov/Blast.cgi & Primer check \\
Blast & Technelysium, South Brisbane AUS & Sequence analysis \\
Chromas Lite 2.1 & NEB, Ipswich USA & Restriction digestion \\
Double Digest Finder & http://www.ensembl.org & Genome browser \\
e!Ensembl & Thomson Reuters, New York USA & Literature management \\
Endnote X1.0.1 & http://www.ncbi.nlm.nih.gov/genbank/ & Genbank accession \\
GenBank & Promega, Mannheim GER & number \\
Glomax® Software & HCR \\
Image J & hational Institutes of Health & Western blot \\
Ligation Calculator & duesseldorf.de/Lig_Input.html & quantification \\
LSM Image Browser & Carl Zeiss, Jena GER & Ligation \\
Microsoft Office & Microsoft, Unterschleißheim GER & secondary antibody \\
MultAlin & http://multalin.toulouse.inra.fr/multalin & decision \\
Photoshop CS3 & Adobe, Munich GER & Word, Excel, Powerpoint \\
& & alignments \\
UDS quantification
\end{tabular}


Pubmed

Reverse Complement

Sigma Blot

Tm caculator

Translate tool

Vector NTI http://www.ncbi.nlm.nih.gov/pubmed http://www.bioinformatics.org/sms/rev comp.html

Systat Software, Erkrath GER

http://www6.appliedbiosystems.com/support/ techtools/calc/

http://web.expasy.org/translate

Life technologies, Darmstadt GER
Literature request

In silico Sequence

translation

Graphs, statistic

annealing temperature

of primers

DNA to protein sequence

translation

Sequence and plasmid design 


\section{Methods}

\subsection{Microbiological Methods}

\subsubsection{Autoclavation and sterilization}

Thermostable buffers and solutions, as well as bacterial media, microbiotically contaminated Erlenmeyer flasks and laboratory S1 waste, were autoclaved for 20 minutes at $121^{\circ} \mathrm{C}$ and three bar. Thermally instable buffers and liquids were sterile filtered. Glass pipettes and miscellaneous glass was washed in a dish washer $\left(40^{\circ} \mathrm{C}\right.$, program $\left.\mathrm{F}\right)$ and subsequently sterilized and dried at $190^{\circ} \mathrm{C}$ and $70^{\circ} \mathrm{C}$ for 4 hours, respectively. Prior to the use in cell culture all glass pipettes were plugged with wadding. Plastic pipette tips were autoclaved for 20 minutes at $121^{\circ} \mathrm{C}$ and three bar and subsequently dried at $70^{\circ} \mathrm{C}$ for 4 hours.

\subsubsection{Culture and storage of E. coli}

Liquid Escherichia coli (E. coli) cultures were grown overnight at $37{ }^{\circ} \mathrm{C}$ in lysogeny broth (LB) and under aerobic conditions in an incubator shaker. For small volumes $(4 \mathrm{ml})$ conical $15 \mathrm{ml}$ tubes were used at 200rpm, for large volumes $(100 \mathrm{ml})$ Erlenmeyer flasks at 180rpm. LB liquid medium or LB agar plates were supplemented with antibiotics according to the required resistance for plasmid amplification (ampicillin $100 \mu \mathrm{g} / \mathrm{ml}$, kanamycin $50 \mu \mathrm{g} / \mathrm{ml}$ ). E. coli cultures intended for competent cells (see chapter 3.1.4) were grown without any antibiotics until the optical density (see chapter 3.1.3) indicated for achievement of the logarithmic growth phase. LB media were prepared according to the manufacture's assignment. For long-term storage, $800 \mu \mathrm{l}$ of an $E$. coli o/n culture was trenchantly mixed with $200 \mu \mathrm{l} 100 \%$ glycerol and stored at $-80^{\circ} \mathrm{C}$.

\subsubsection{Photometric measurement of optical density of bacterial cultures}

Calculation of the optical cell density (OD) of bacterial cultures was done at $600 \mathrm{~nm}$ in $1 \mathrm{ml}$ volume using a Spectrophotometer and the knowledge of the Lambert-Beer law. A value of 0.5 at the $\mathrm{OD}_{600}$ accounted for the achievement of the logarithmic growth phase. Pure autoclaved LB medium served as blank.

\subsubsection{Preparation of chemically competent Escherichia coli}

The E. coli strain DH5 $\alpha$ was used as host for plasmid amplifications (see chapters 3.2.2.2, 3.3.4) and hence for the preparation of chemically competent cells. The underling mechanism is unknown in 
detail. It involves the perforation of bacterial cell-wall and -membranes and its positive charging which enables these cells to uptake negatively charged DNA.

A single colony of plated $\mathrm{DH} 5 \alpha$ cells was picked to inoculate a $10 \mathrm{ml}$ overnight culture (see chapter 3.1.2). This preparatory culture was poured into $190 \mathrm{ml}$ of autoclaved LB medium on the next day and the cell suspension grew until $O_{600}$ reached 0.5 (see chapter 3.1.3). Following, the cells and used buffers were kept at $4^{\circ} \mathrm{C}$ all the time. After centrifugation [4000rpm, 10min] the pellet was resuspended in $25 \mathrm{ml}$ of $100 \mathrm{mM} \mathrm{MgCl} 2$ solution and incubated on ice [5min]. The dissolution was centrifuged again [4000rpm, $10 \mathrm{~min}$ ] and the pellet was resuspended in $5 \mathrm{ml}$ of $100 \mathrm{mM} \mathrm{CaCl}_{2}$ solution for incubation $\left[4^{\circ} \mathrm{C}, 20 \mathrm{~min}\right]$ and subsequently centrifuged again. Finally, the pellet cells were resuspended in $1 \mathrm{ml}$ calcium-glycerol buffer and immediately frozen in aliquots of $50 \mu \mathrm{l}$ using liquid nitrogen. In this vein produced competent cells were stored at $-80^{\circ} \mathrm{C}$ and used for transformation with a transformation efficacy of about $10^{5}$ to $10^{6}$ colonies per $\mu \mathrm{g}$ DNA (see chapter 3.1 .5 ).

\subsubsection{Transformation of E. coli}

Transformation is the genetic alteration of a prokaryotic cell by transfer of foreign DNA and used for amplification of recombinant plasmid DNA (Hanahan, 1983).

Chemically competent DH5 $\alpha$ (see chapter 3.1.4) were defrosted on ice and an appropriate amount of plasmid DNA was added (100ng for re-transformation of plasmid preparations (see chapter 3.2.2.2) or $5 \mu \mathrm{l}$ of quick-change (see chapter 3.2.3.3) or ligation (see chapter 3.2.3.3.3) reactions). After an incubation time of $30 \mathrm{~min}$ on ice, which leads to the stable attachment of the nucleic acid to the cell membrane, a heatshock was performed $\left[45 \mathrm{sec}, 42^{\circ} \mathrm{C}\right]$ with a subsequent chill-on-ice step $(2 \mathrm{~min})$ to increase the uptake of DNA into the cells. In order to cure the perforated bacteria $450 \mu$ l of liquid LB was added, followed by an incubation of $1 \mathrm{hr}$ at $37^{\circ} \mathrm{C}$ under shaking conditions. Finally the whole transformation reaction (quick-change mutagenesis and ligation) or a small portion (retransformation, dilution plating to pick single colonies) was plated on LB agar dishes supplemented with the according antibiotic (resistance is encoded on the used plasmid) to achieve selection of successfully transformed $E$. coli clones. 


\subsection{Molecular methods}

\subsubsection{Purification and concentration of nucleic acids}

Purification and concentration of nucleic acids was either achieved by ethanol precipitation (see chapter 3.2.1.1) or the use of the 'Gel extraction and PCR Clean Up' Kit according to the manufacturer's instructions or a combination of both.

\subsubsection{Precipitation of nucleic acids}

Polar molecules dissolve well in also polar resolvents. Like water, DNA is a polar molecule because of its highly negatively charged sugar-phosphate backbone. Water binds positively charged ions, e.g. $\mathrm{Na}^{2+}$, and solubilises salt very well and a DNA molecule has the same properties. Ethanol is much less polar compared to water, which means that adding ethanol to solution disrupts the bindings of charges by water. If enough of it is added (above $64 \%$ of ethanol in solution), the electrical attraction between phosphate groups and any positive ions present in solution becomes strong enough to form stable ionic bonds (Green et al, 2012).

A tenth part of the sample volume of high salt sodium acetate concentrations (3M) and 2.5 times sample volume of $96 \%$ ethanol (analytical grade) were added to the DNA containing sample. After intense vortexing and 30min of high speed centrifugation (14000rpm) the supernatant was discarded. Next, $300 \mu$ l of $70 \%$ ethanol was added to wash the pellet followed by centrifugation [10min 14000rpm]. Subsequently the ethanol was pipetted off and the pellet was dried [5min, $\left.42^{\circ} \mathrm{C}\right]$ on a thermoblock with tubes open. Finally the pellet is resuspended in water.

After plasmid preparation (see chapter 3.2.1.1) the DNA containing solution is precipitated with 2propanol (70\% final concentration) instead of sodium acetate and Ethanol. Vortexing and washing steps stayed equal.

\subsubsection{Concentration determination}

Measurement of the concentration of nucleic acids was done at an absorption of $260 \mathrm{~nm}$ in a volume of $2 \mu \mathrm{l}$ using the NanoVue ${ }^{\circledR}$. Autoclaved bidest water served as blank, the concentration was calculated by the device using standards conversion factors for DNA, RNA and oligonucleotides (50, 40 , and 20 , respectively). The quotient $O D_{260} / O D_{280}$ was used as a purity control and always above 1.6 . 


\subsubsection{Isolation of nucleic acids}

\subsubsection{Extraction of nucleic acids from agarose gels}

Prior to cloning (see chapter 3.2.3.4), DNA fragments of different size were extracted and purified from 1\% agarose gels (see chapter 3.2.2.1) using the NucleoSpin ${ }^{\circ}$ Gel extraction and PCR clean up Kit by Machery and Nagel according to the instructions of the manufacturer. The DNA containing bands were uncovered due to Ethidium bromide intercalation and short time visualization by UV light and subsequently cut out with a clean scalpel.

\subsubsection{Plasmid DNA preparation}

Plasmid preparation from bacteria was first described in the nucleic acids research journal (Birnboim \& Doly, 1979) as rapid alkaline extraction procedure and is, in principle, unaltered used in laboratories since that time. It makes use of the unequal behaviour of different DNA molecules of different size in solution after denaturation. The harvested $E$. coli cultures (see chapter 3.1.2) in mini $(4 \mathrm{ml})$, midi $(100 \mathrm{ml})$ or maxi $(400 \mathrm{ml})$ format were centrifuged [4000rpm, 10min] and in a first step gently treated with RNase prior to the cell lysis step under basic conditions (e.g. by the use of $\mathrm{NaOH}$ ). By the incubation at room temperature [5min] cellular proteins as well as dsDNA (chromosomal and plasmids) were denatured and nucleic acids consequently strand separated. The following neutralization step involves an acidic buffer (e.g. potassium acetate) which leads to renaturing of small molecules (e.g. plasmids) and the precipitation of large molecules (genomic DNA, Proteins, and membrane components). This white mass is sedimented by centrifugation and the supernatant is transferred to glass silica columns which are able to bind the intact plasmid DNA. These columns as well as the required buffers and enzymes were provided by Machery + Nagel. The suitable Kit for each culture volume was used according to the manufactures manuals. After elution in appropriate volumes precipitation was carried out using 2-propanol (see chapter 3.2.1.1). The concentration of the plasmid DNA was determined like described (see chapter 3.2.1.2).

\subsubsection{Preparation of eukaryotic nucleic acids}

Total RNA and genomic DNA were isolated from $2 * 10^{6}$ to $5 * 10^{6}$ wildtype or patient fibroblast cells (see chapter 3.3.1). RNA samples were further processed during cDNA synthesis (see chapter 3.2.3.1) in an mRNA specific manner.

For RNA isolation cells were centrifuged, the supernatant was decanted and the pellet resuspended in $600 \mu \mathrm{l} \mathrm{RLT}$ buffer (supplemented with $1 \%(\mathrm{v} / \mathrm{v}) \beta$-Mercaptoethanol) and, as the case may be, stored at $-80^{\circ} \mathrm{C}$ for maximally a few days. This buffer was provided by Qiagen in the RNeasy Mini Kit and 
further processed by following the instructions of the appendent handbook. The optional DNase digestion was obligatorily done using the RNase free DNase Set provided by Qiagen.

Genomic DNA was prepared using the QIAamp DNA Blood Kit and the corresponding manufacturer's instructions. The concentration of both eukaryotic nuclei acids was determined like described (see chapter 3.2.1.2).

\subsubsection{Enzymatic manipulation of nucleic acids}

\subsubsection{Complementary DNA (cDNA) synthesis}

Prepared RNA samples (see chapter 3.2.2.3) were used for cDNA synthesis using the Revert Aid H Minus First Strand cDNA Synthesis Kit according to Fermentas' instructions. The predesigned 18-mer oligonucleotides $d T$ anneal specifically with the poly(A)-tail of messenger RNA (mRNA). Thus only stably transcribed genes are part of the cDNA pool generated by the reverse transcriptase polymerase (virus derived polymerases).

Maximally $5 \mu \mathrm{g}$ of total RNA were used for pre-annealing of $0.5 \mu \mathrm{g}$ oligo $\mathrm{dT}$ [ $5 \mathrm{~min}, 70^{\circ} \mathrm{C}$ ] in a reaction volume of $20 \mu \mathrm{l}$. After $1 \mathrm{~min}$ on ice the premix (buffer, RNase inhibitor, dNTPs) was added an incubated for $5 \mathrm{~min}$ at $37^{\circ} \mathrm{C}$. The subsequently added reverse transcriptase polymerized at for $60 \mathrm{~min}$ at $42^{\circ} \mathrm{C}$ and additionally for $10 \mathrm{~min}$ at $72^{\circ} \mathrm{C}$. In that fashion synthesized cDNA was stored at $-20^{\circ} \mathrm{C}$ prior to the use in PCR reactions (see chapter 3.2.3.2).

\subsubsection{Polymerase chain reaction (PCR)}

The Polymerase chain reaction was first described in 1986 (Mullis et al, 1986). It is until now routinely used in laboratories for almost exponential DNA amplification of a specific sequence. It involves 3 consecutive steps which are repeated several times (cycles, n): strand separation (denaturation), primer annealing and elongation (polymerization).

The target sequence is encoded on the added template which can be genomic dsDNA, cDNA or even whole cells (colony PCR). Prior to the Primer annealing step an obligatory denaturation step is performed to trigger strand separation. For each amplification reaction of a certain DNA fragment two primers are designed for either leading or lagging strand (double stranded cDNA exists from the beginning of the $2^{\text {nd }}$ amplification round). Primer for sequencing (see chapter 3.2.4.2) and cloning (see chapter 3.2.3.4) design was performed regarding six properties:

- 18 to 24 nucleotides in length with a GC-content of $40 \%$ to $60 \%$

- Strong 5' (GC-rich), weak 3' (AT-rich) binding

- Melting temperature between 50 to $60^{\circ} \mathrm{C}$ (insofar as possible)

- No primer dimer or palindromic (self annealing) sequence

- Uniqueness of the primer sequence (BLAST) 
Processive DNA polymerases with high accuracy derived from thermostable organisms are used to polymerize dNTPs, starting from the 3' $\mathrm{OH}$-ends of specifically designed primer oligonucleotides according to the complementary template sequence. The denaturation and elongation time, if necessary also the annealing temperature, were set according to manufacturer's specifications. With the beginning of the $3^{\text {rd }}$ cycle the PCR works with an exponential $\left(2^{n}\right.$, optimal conditions postulated) rate of amplification of a certain DNA fragment with a distinct size (number of base pairs, see chapter 3.2.4.1).

Reactions were done in a volume of $50 \mu \mathrm{l}$ and an average PCR program was configured in the following fashion (insofar as applicable):

- $\quad 98^{\circ} \mathrm{C}, 30$ s initial denaturation

- $\quad 98^{\circ} \mathrm{C}, 20$ s denaturation

$50-60^{\circ} \mathrm{C}, 20$ s primer annealing

$72^{\circ} \mathrm{C}, 3.5$ min elongation 35 cycles

- $\quad 72^{\circ} \mathrm{C}, 10 \mathrm{~min}$ remaining elongation

\subsubsection{Site directed mutagenesis (Quick change)}

Site directed mutagenesis is a PCR application (see chapter 3.2.3.2) and used for specific mutagenesis of one or two adjacent bases of a protein coding DNA sequence in order to introduce point mutations and thus change the amino acid sequence. $125 \mathrm{ng}$ of cloned plasmid vectors (see chapter 3.2.3.4) were used as template. A complementary primer pair was designed to anneal as $>30 \mathrm{mer}$, at least 15 nucleotides flanked up- and downstream of the position of interest, according to leading (for) and lagging (rev) strand sequence. The PCR reaction was performed with an extended elongation time in order to amplify the whole plasmid (18 cycles). After the PCR reaction took place, a Dpnl restriction digestion was performed $\left[20 \mathrm{U}, 2 \mathrm{~h}, 37^{\circ} \mathrm{C}\right.$ ] followed by heat inactivation [20min, $80^{\circ} \mathrm{C}$ ]. Dpnl is a type II restriction endonuclease that is able to cut methylated DNA, thus only the (not mutated) template DNA. Bacterial transformation (see chapter 3.1.5) was done for clone selection.

\subsection{Restriction digestion}

Either DNA fragments or plasmid vectors were digested with appropriate restriction enzymes according to the design of the cloning strategy (see chapter 3.2.3.4), in volumes of $50 \mu \mathrm{l}$ or $100 \mu \mathrm{l}$, respectively. Suitable buffers for single or double digestions were used according to the enzyme providing company's description. Restriction digestions were performed $\mathrm{o} / \mathrm{n}$ with 30 units per enzyme at $100 \%$ activity in the relevant buffer. Lower activities led to higher input of enzyme. Finally the reactions were precipitated (see chapter 3.2.1.1) and the DNA resuspended in an appropriate volume prior to gel extraction $(50 \mu \mathrm{l}$, see chapter 3.2.2.1). 


\subsection{Dephosphorylation}

Digested vectors (see chapter 3.2.3.3.1) were dephosphorylated to prevent re-ligation of the linearized vector. $20 \mu \mathrm{g}$ of the plasmid DNA was used for the treatment with calf intestinal alkaline phosphatase (CIAP). Reactions were carried out in a volume of $50 \mu \mathrm{l}$ and unit calculation was done according to the manufacturer's instructions. The reaction was incubated $\left[37^{\circ}, 1 \mathrm{~h}\right]$ following enzyme inactivation $\left[65^{\circ} \mathrm{C}, 15 \mathrm{~min}\right]$. Afterwards, ethanol precipitation (see chapter 3.2.1.1) was done.

\subsection{Ligation}

DNA strands polymerize by covalent phosphodiester bonds between free $3^{\prime}$ hydroxyl and 5' phosphate ends of adjacent nucleotides. The used T4 DNA ligase catalyzes this reaction in an ATPdependent manner and is used to conjugate linearized vectors and inserts (see chapters 3.2.3.3.1, 3.2.3.4). The molecular vector: insert ratio of $1: 3$ was calculated with the help of an online tool and either 20 or 100ng of vector were used depending of the amount of insert DNA available. Reactions were carried out using $20 \mathrm{U}$ ligase in a reaction volume of $20 \mu \mathrm{l}$ either $1 \mathrm{~h}$ at room temperature or overnight at $16^{\circ} \mathrm{C}$.

\subsubsection{Molecular cloning}

Molecular cloning involves several subsequent steps and leads to artificial recombinant DNA plasmids used for overexpression of certain proteins in functional assays (see chapter 3.5). The cloning strategy, developed with the help of vector NTI, depends on the nature of the MCS of the vector and the sequence of the DNA molecule to insert. To make sure the used restriction enzymes do not cut inside the gene the whole sequence has to be checked for the absence of the appropriate restriction site(s). Forward and reverse primers were designed to amplify the DNA sequence of interest by PCR (see chapter 3.2.3.2) and restriction sites were attached to the $5^{\prime}$ end of the primers according to the need of the vector MCS. After vector (see chapter 3.2.2.2) and DNA fragment (insert) preparation (see chapter 3.2.2.1) the yielded nucleic acids were digested (see chapter 3.2.3.3.1). The linearized vector was dephosphorylated (see chapter 3.2.3.3.2) and ligated with the insert DNA (see chapter 3.2.3.3.3). After transformation (see chapter 3.1.5) single colonies were analyzed by (colony) PCR (see chapter 3.2.3.2) and sequencing (see chapter 3.2.4.2).

\subsubsection{Analysis of DNA}

\subsubsection{Agarose gel electrophoresis}

Agarose gel electrophoresis is a tool to separate DNA molecules according to their shape (supercoiled, coiled or linear) and most importantly size (bp). Nucleic acids are highly negative charged and migrate in an electric field towards the anode through the homogeneous agarose gel 
matrix with a certain speed proportional to their lengths. DNA fragments either yielded by PCR (see chapter 3.2.3.2) or restriction digestion (see chapter 3.2.3.3.1), and also linearized vectors, were utilized in agarose gels of different concentrations $(\mathrm{w} / \mathrm{v})$ of $0.7 \%$ to $2.0 \%$ according to their expected size, e.g. $5 \mathrm{kbp}$ plasmids or small 50bp fragments, respectively. Gel electrophoresis was used for analytical questions $(10 \mu$ l volume per probe) or preparative application $(50 \mu l$ volume), the latter prior to gel extraction (see chapter3.2.2.1) and ligation (see chapter 3.2.3.3.3). At least one convenient DNA ladder $(4 \mu \mathrm{l})$ was used in a single lane for size discrimination of the different fragments. Before loading of samples on the gel they were mixed with an appropriate volume of $6 x$ loading dye. TBE was used for gel preparation and as running buffer and consequently to establish the electric field, voltage $70-130 \mathrm{~V}$, between cathode and anode. The gels were incubated in a $0.01 \%(\mathrm{v} / \mathrm{v}$ ) ethidium bromide bath (a DNA intercalating agent) for $5 \mathrm{~min}$. The uptake of this fluorescent dye and hence DNA fragments were visualized using a 312nm UVA lamp.

\subsubsection{Sequencing}

Sequencing was developed as chain termination method by Frederick Sanger and colleagues (Sanger et al, 1977). In principle, it works like a PCR (see chapter 3.2.3.2) and uses, beside normal dNTPs, in a proportion of $1 \%$ dideoxynucleotides (ddATP, ddCTP, ddGTP, or ddTTP) which lack the $3^{\prime}$ hydroxyl group and hence are deficient in polymerization. The traditional version involves PAA-gel electrophoresis for separation of the randomly chain disrupted DNA fragments of each reaction with one of ddNTPs. According to the fragment length, positions of the respective nucleotide can be read out. The modern version of Sanger sequencing utilizes labeled ddNTPs with 4 different fluorescent dyes all emitting light at diverse spectral colors. This allows polymerization reactions for all four nucleotides in one tube and discrimination by excitation of these dyes by certain wavelengths using a capillary sequencer with a laser and fluorescence detector.

Reactions were carried out in a volume of $10 \mu$ l using beside the appropriate volume of buffer and $1 \mu l$ Big Dye Ready reaction Mix (of the corresponding Kit; incl. polymerase), 300-500ng of plasmid template DNA ( $\sim 9 \mathrm{kbp})$ and $5 \mathrm{pmol}$ of the required sequencing primer. A standard sequencing reaction was performed with the following program:

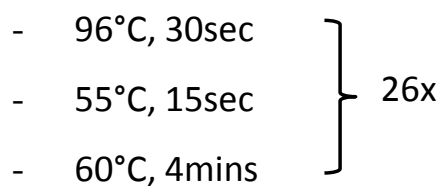

Afterwards, the reactions were alcohol precipitated (see chapter 3.2.1.1) and the pellet was gathered in $10 \mu \mathrm{l} \mathrm{Hi-Di} \mathrm{formamide.} \mathrm{The} \mathrm{DNA} \mathrm{sequence} \mathrm{(chromatograph)} \mathrm{was} \mathrm{analyzed} \mathrm{with} \mathrm{Chromas} \mathrm{Lite.}$ 


\subsection{Cell culture methods}

\subsubsection{Culture of cells and preparation of primary cell cultures}

Adherent primary fibroblasts were obtained from skin punch biopsies of healthy donors (wildtype) or patients (XP172MA) ordered at the Coriell Cell Repositories (XP20BE). Hair, fat, and keratinocytes were removed from human skin probes which were then cut into small pieces of about 1-4 $\mathrm{mm}^{2}$. These slices were arranged all over the entire area of a $175 \mathrm{~cm}^{2}$ culture flask with gaps of several centimeters in between in every direction. After short desiccation incubation [15 min] at room temperature with open lid under the sterile bench, $25 \mathrm{ml}$ of DMEM cell culture media (complete) was gently pipetted into the flask followed by incubation for at least one week without touching. All cultures, also purchased XP20BE cells, were incubated in a humified atmosphere at $37{ }^{\circ} \mathrm{C}$ and $5 \%$ $\mathrm{CO}_{2}$ until they reached confluency. Afterwards they were trypsinized at $37^{\circ} \mathrm{C}$ for 5 min with an appropriate amount of trypsin (ground had to be covered). This reaction was stopped using 2.5-fold FBS-containing DMEM media followed by centrifugation [1000 rpm, 5min] and pellets resuspended in adequate DMEM or PBS volumes. The cells were either used for freezing (see chapter 3.3.2), cell counting (see chapter 3.3.3), or functional assays (see chapter 3.5). The re-seeding of primary fibroblast was performed with a cell density of at least $40 \%$ confluency.

\subsubsection{Freezing and thawing cells}

In order to freeze primary fibroblasts for later thawing and re-seeding only early passages (as far as passage 7) of confluent cell culture flasks were trypsinized at $37^{\circ} \mathrm{C}$ for 5 min with an appropriate amount of trypsin (ground had to be covered), centrifuged [1000 rpm, 5min] and resuspended in $1 \mathrm{ml}$ DMEM (complete). Afterwards $0.5 \mathrm{ml}$ were mixed with $0.5 \mathrm{ml}$ freezing medium in precooled cryo tubes and after scarfing subsequently frozen cautiously o/n in cryo $1^{\circ} \mathrm{C}$ cooler filled with 2-propanol at $-80^{\circ} \mathrm{C}$ (approximately $1^{\circ} \mathrm{C} / \mathrm{min}$ ). On the next day the cryo tubes were transferred into cryo boxes in liquid nitrogen for permanent storage at $-196^{\circ} \mathrm{C}$.

For thawing of cells the stored cryotubes were gently de-frosted at room temperature and directly transferred into 10ml DMEM (complete) and subsequently centrifuged. The supernatant was decanted, to dispose the DMSO in the freezing medium, and the sediment was resuspended in an appropriate volume of DMEM (complete) prior to re-seeding in cell culture flasks or cell counting (see chapter 3.3.3).

\subsubsection{Cell counting}

To perform functional assays (see chapter 3.5) cells had to be seeded in a certain density in 24-well plates (approximately 40-50\% confluency) and therefore the cell number was calculated using a 
Neubauer cell counting chamber. Therefore $10 \mu \mathrm{l}$ of the cell suspension was mixed with $90 \mu \mathrm{l}$ trypanblue by pipetting several times, a glass cover slip was put on the Neubauer chamber and $10 \mu \mathrm{l}$ were of the mixture were used for counting. Four large squares (each one $\mathrm{mm}^{2}$ ) were counted, in total a volume of four $\mathrm{mm}^{3}$, and the cell number was multiplied by 2500 to estimate the total number per $\mathrm{ml}$ and again by 10 because of the dilution factor in trypanblue. The Neubauer chamber was cleaned in water and $70 \%$ ethanol.

\subsubsection{Transient transfection}

Transient transfections were carried out using the nonliposomal lipid Attractene, a transfection reagent provided by Qiagen. It forms complexes with plasmid DNA which are assimilated by eukaryotic cells like micelles via endocytosis. Therefore cells were seeded, 20000 cells per well in 24well format or $9 * 10^{5}$ in petridishes, respectively (see chapter 3.3.1), and incubated $\mathrm{o} / \mathrm{n}$ for adhering. On the next day total either $250 \mathrm{ng}$ or $4 \mu \mathrm{g}$ of one or more plasmids were diluted in DMEM to either $60 \mu \mathrm{l}$ or $300 \mu \mathrm{l}$ final volume, either $0.6 \mu \mathrm{l}$ or $15 \mu \mathrm{l}$ of Attractene was added and instantly vortexed for several seconds in either 24-well or petridish format, respectively. During the incubation time of 15 minutes at room temperature the wells were soaked and filled with 500 $\mu$ fresh DMEM (complete). Afterwards the plasmid DNA containing transfection mix was put drop wise onto the cells and incubated in a humified atmosphere $\left[5 \% \mathrm{CO}_{2}, 37^{\circ} \mathrm{C}\right]$ for five to six hours. The medium was removed and the wells filled with $\sim 1 \mathrm{ml}$ of fresh complete DMEM and incubated two or three days, to allow (over-) expression of the recombinant proteins (see chapter 4.2.1).

\subsection{Biochemical methods}

\subsubsection{Preparation of whole cell protein lysates}

After harvesting using trypsin (see chapter 3.3.1), the cells were washed twice with PBS and a subsequent centrifugation step [5Min, 4000rpm]. The resulting pellet was resuspended in a small volume of a PMSF and proteinase stop containing PBS buffer. In an iterative cycle of three times repeating a freeze-thaw step, in the liquid nitrogen bath and on ice, respectively, was performed. This step lyses the cells and prepares the proteins. Subsequently centrifugation follows [10min, 14 000rpm, $4^{\circ} \mathrm{C}$, which leads to the pelletization of cell debris and the dissolving of the proteins in the buffer. The supernatant was transferred into a new cup and photometrically quantified by the Bradford method (see chapter 3.4.2) (Bradford, 1976). The protein lysates were adjusted with an $3 \%$ SDS and $10 \% \beta$-mercaptoethanol containing SDS page sample buffer and boiled at $95^{\circ} \mathrm{C}$ for $10 \mathrm{~min}$. Afterwards the yield was stored at $-20^{\circ} \mathrm{C}$. 


\subsubsection{Bradford protein quantification method (Bradford, 1976)}

For photometric quantification of protein concentrations, $1 \mu$ l of protein lysate (see chapter 3.4.1) was mixed with the ready to use Bradford solution according to the manufacture's advice and photometrically measured $\left(\mathrm{OD}_{595}\right)$. Concentrations were normalized with the help of a BSA calibration curve (serial dilutions, $0-10 \mu \mathrm{g} / \mathrm{ml}$ ).

\subsubsection{Polyacrylamide gel electrophoresis (SDS-Page) (Laemmli, 1970)}

Proteins were separated by size in an electric field using SDS-Page (polyacrylamide gel electrophoresis). The SDS molecule, a negatively charged strong detergent agent, disturbs via its aliphatic tail the tertiary structure of proteins and transfers a negative overall charge to each protein. This enables a fractionization mainly by size according to the molecular weight ( $\mathrm{kDa}$ ) during electrophoresis. Prior to each supply of the samples (see chapter 3.4.1) to the gel, the probes were boiled $\left(95^{\circ} \mathrm{C}\right)$ for $5 \mathrm{~min}$, again. The Biorad Mini Protean Tetra Cell system with a precast $4 \%$ to $15 \%$ polyacrylamide gradient Mini TGX gels were used according to manufacturer's instructions.

\subsubsection{Western Blotting}

For immunochemical analysis the separated proteins in a SDS-Page gel (see chapter 3.4.3) are transferred ("blotted") onto a nitrocellulose membrane. In analogy to the technically related method used for blotting of RNA onto membranes, developed by the British biologist Edwin Southern, the method described here was named "western blot".

The Biorad Transblot Turbo System was used to perform a semidry-blot with a current of $0.6 \mathrm{~A}$ and maximal voltage of $12 \mathrm{~V}$ for $10-15 \mathrm{~min}$. A Ponceau S staining of the SDS gel was performed to check for the integrity of the protein transfer. After blotting, the nitrocellulose membranes were incubated with blocking solution (Western Breeze kit) for $1 \mathrm{~h}$. As well as mono- and polyclonal antibodies diluted in blocking solution (concentrations see Table 4) were incubated together with the membrane overnight at $4^{\circ} \mathrm{C}$ (shaking). Afterwards the membranes were washed four times for $5 \mathrm{~min}$ with washing solution and incubated with the secondary antibody (anti-mouse or anti-rabbit) for $30 \mathrm{~min}$ at RT. Further washing (four times for $5 \mathrm{~min}$ and rinsed two times with aqua bidest) subsequently follows the adding of the chemiluminescent substrate. The LAS4000 was used for the detection of the chemiluminescent signal (Image reader for $30 \mathrm{sec}-5 \mathrm{~min}$ using the Increment Super setting). Bands were densiometrically quantified using Image J. Normalization was performed using the $\beta$-Actin housekeeper. 


\subsection{Functional Methods}

\subsubsection{Host Cell Reactivation assay (HCR)}

The Host Cell Reactivation (HCR) reporter gene assay measures the Nucleotide excision repair (NER) in actively transcribed genes. This reporter gene assay deploys the defect expression of (unrepaired) (UV-) damaged cDNA sequences (Protic-Sabljic \& Kraemer, 1985) as readout for the NER capacity and is by default used for complementation group assignment of XP patients. Moreover, it can be used for either DNA repair capacity assessment of different fibroblast cells or, like it was done during this thesis, of transfected (repair-) gene variants in NER deficient patient cells.

XPG deficient XP2OBE cells (see chapter 1.4) and, as a positive control, wildtype fibroblasts were seeded (see chapter 3.3.1) and (among other complementation plasmids) transfected (see chapter 3.3.4) with plasmids coding for firefly (100ng) and renilla (50ng) luciferases. The plasmid coding for firefly luciferase is divided into two fractions prior to transfection. One fraction is irradiated with 750 $\mathrm{J} / \mathrm{m}^{2}$ of UVC light, a second fraction stays untreated. For irradiation, the plasmid-containing solution $(250 \mathrm{ng} / \mu \mathrm{l})$ was drop wise ( $3-4 \mu \mathrm{l}$ per drop) distributed over the surface of the petridish. The always unirradiated renilla luciferase serves as control for normalization of transfection efficacy and transcriptional activity of the respective gene variants. Additionally, a complementation plasmid (100ng) was added which was either an empty vector (negative control in XP20BE or in wtfibroblasts, too keep luciferase plasmid concentrations unaltered), wt-XPG or an XPG-variants (see chapter 4.2.1).

After an incubation time of three days, which allows sufficient repair of the photoproducts created by UVC-irradiation and protein expression of the luciferases, the wells were washed with $1 \mathrm{ml} \mathrm{PBS}$ and lysed in $80 \mu \mathrm{l}$ of a special lysis-buffer provided by Promega's HCR kit [45min, RT]. Thereafter, the cells were scraped off and $40 \mu \mathrm{l}$ of each well was used for luminescence measurements in a white Glomax $^{\mathrm{TM}} 96$ microplate. Here $80 \mu \mathrm{l}$ of each luciferase substrate are subsequently added and luminescence is quantified using the Glomax ${ }^{\mathrm{TM}}$ luminometer (in RLUs). The buffers provided by Promega are designed for successive measurement of both luciferases, meaning the firefly substrate (beetle luciferine) contains a stop-solution to quench the luminescence after the measurement, before the renilla substrate (coelenterazine) was added. Thus it is possible to measure both luciferase activities in one well.

The relative repair capacity is estimated using this formula:

$$
\text { repair }(\%)=\frac{\text { mean (irradiated firefly/renilla per well) }}{\text { mean (un-irradiated firefly/renilla per well) }} \times 100
$$


The estimated mean values depicted in the figures derived from at least nine independent experiments. The errorbars were calculated using error propagation of the SEM of each fraction (normalized damaged and undamaged). For statistical analysis the student's T-Test was performed.

\subsubsection{Unscheduled DNA Synthesis (UDS)}

Unscheduled DNA Synthesis (UDS) was first published in the late 1960s (Cleaver, 1968; Painter \& Cleaver, 1969) and is a method for (quantitative) measurement of DNA repair synthesis in nondividing cells. It used incorporated radioactive labeled nucleotide analogs as marker for DNA synthesis after damage and was since that time a tool for complementation group assignment of XP patients (Lehmann \& Stevens, 1980). This method was further improved (Limsirichaikul et al, 2009) and nowadays utilizes the terminal alkyne EdU, a thymidine analogs which is able to covalently bind to an Alexa Fluor ${ }^{\circledR} 488$ azide via a copper-catalyzed click reaction. This in turn can be visualized by e.g. a fluorescence microscope (Chehrehasa et al, 2009).

On the first day 20000 cells have been seeded (see chapter 3.3.1) on round glass cover slips in 24well plates and were incubated until the next day $\left[37^{\circ} \mathrm{C}, 5 \% \mathrm{CO}_{2}\right]$. All buffers and solutions used during the procedure had to be prepared according to manufacturer's instruction (Click-iT EdU Alexa Fluor 488 Imaging Kit). 48 hours after transfection (see chapter 3.3.4) the UDS assay starts directly with the UVC irradiation $\left(20 \mathrm{~J} / \mathrm{m}^{2}\right)$ of the cells to induce UV damages in the genomic DNA. Therefore the medium was soaked off the wells and the cells were washed once with PBS and soaked again. Subsequently, the irradiation was carried out without any liquid on the cells to avoid refraction of the UV light. EdU was added to DMEM without any accessories, mainly without FBS, in a concentration of $10 \mu \mathrm{M}(1: 1000)$ and $500 \mu \mathrm{l}$ were put on the cells per well. During the following incubation $\left[2 \mathrm{hrs}, 37^{\circ} \mathrm{C}\right.$, $\left.5 \% \mathrm{CO}_{2}\right]$ the thymidine analogs were incorporated into the DNA in the course of (repair) synthesis. After washing the cells with PBS three times they were prefixed with $0.5 \mathrm{ml}$ sucrose-solution per well for $20 \mathrm{~min}$ at room temperature. After washing with PBS (3 times) cells were blocked with $10 \%(\mathrm{v} / \mathrm{v})$ FBS in PBS for $30 \mathrm{~min}$ at $37^{\circ} \mathrm{C}$. During this blocking step, maximally $15 \mathrm{~min}$ prior to use, the reaction cocktail for the copper-catalyzed click reaction was composed according to protocol provided by life technologies (in the dark). In case of sole used Alexa Fluor ${ }^{\circledR} 488$ azide (dissolved in 100 $\mu$ l DMSO) 50\% more of it, compared to the amount in the Click-It EdU kit, were added. After soaking the blocking solution, contrary to the manufacturer's instructions, $250 \mu \mathrm{l}$ of the cocktail reaction were used per well and incubated for 30min at room temperature. After washing the cells three times with PBSTween a terminal fixation step is performed with $3.7 \%$ PFA for $20 \mathrm{~min}$ at room temperature. Finally, cells were washed with PBS again three times and the glass cover slips were put upside down onto object slides prepared with one drop, big as a pinhead, of DAPI-containing mounting medium. After 
affixing the glass cover slips onto the object slides, using a clear nail polish, the probes were dried and kept at $4^{\circ} \mathrm{C}$ over night.

For visualization of UDS the object slides were put on a fluorescence microscope and pictures were made using the Axiovision software and appropriate filters for Alexa Fluor ${ }^{\circledR} 488$ and DAPI with an exposure time of $7000 \mathrm{~ms}$ and about $100 \mathrm{~ms}$, respectively. The pictures were automatically merged by the program.

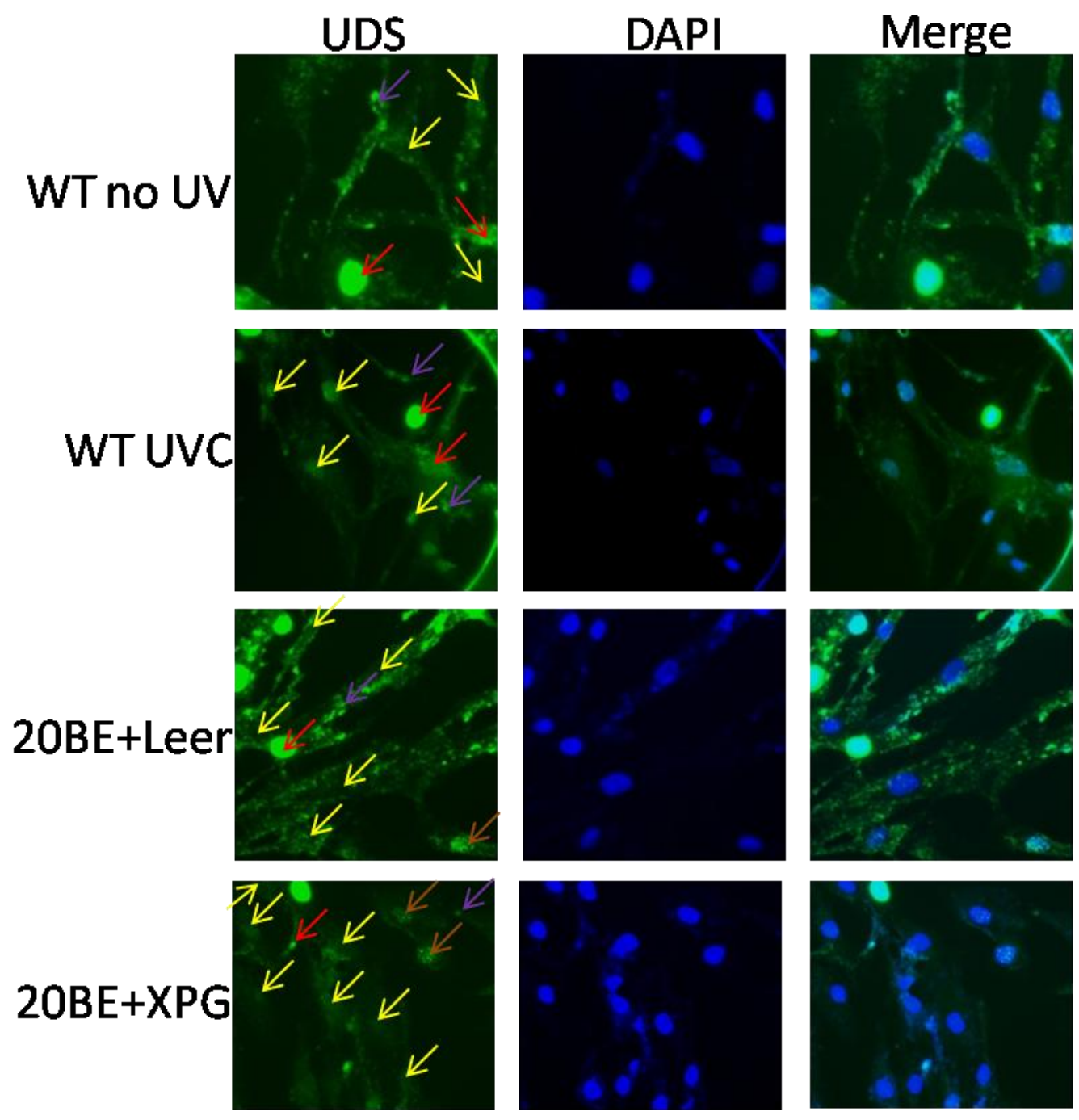

Figure 15: Measurement of Unscheduled DNA synthesis (UDS)

Description: yellow arrows = measurement followed by subsequent cytoplasm fluorescence measurement of the corresponding cell for subtraction. Red arrows = excluded from measurement (S-phase nuclei and unspecific cell structures). Pink arrows = excluded from measurement (missing of DAPI staining). Brown arrows = excluded from measurement due to graining likely marking replication, that just started had started.

Photoshop ${ }^{\circledR}$ CS3 was used for quantification. The monograph (black/white) picture of the FITC channel (Alexa Fluor ${ }^{\circledR} 488$ ) of each slide was used and the gray level per square pixel in nucleus and cytoplasm (auto fluorescence) was estimated per cell and subtracted from each. This gains net 
intensity of DNA repair synthesis. In this context DAPI served as nuclear stain for orientation. Nuclei in s-phase, which had an obviously strong glow, were excluded from the measurement. Each result was quantified as the mean/median whereat minimum 300 cells (nuclei) were analyzed, derived from at least three independent experiments. For statistical analysis the Mann-Whitney-U-Test for abnormally distributed values was used.

\subsubsection{Immunofluorescence}

Indirect immunofluorescence is a widely used technique that utilizes primary antibodies (see Table 4) to detect specific target antigens and, targeting these, fluorescent-labeled secondary antibodies (see Table 5) for visualization (Odell \& Cook, 2013). Target antigens are either conformational epitopes, e.g. the tertiary structure of proteins or structures like DNA UV damages, or linear epitopes, the primary amino acid sequence of certain peptides or proteins.

In order to perform Immunofluorescence analysis adherent fibroblast cells, wildtype or XP20BE, were seeded on glass cover slips in 24-well plates (20 000 cells per well, see chapter 3.3.1) and transfected (see chapter 3.3.4) with the desired XPG-variant or the empty vector on the next day. An incubation time of 48 hours ensures the adequate (over-) expression of the proteins.

Thereafter the conditioned medium was soaked off and preserved for later, the cells were washed once with PBS. Prior to UVC irradiation the cells were wetted with $1 \mu \mathrm{IPBS}$ and an isopore membrane $(5 \mu \mathrm{m})$ was applied onto the cells. This membrane contains tiny holes whereby punctual UV damages (spots) are introduced into the cells nuclei (DNA), which serve e.g. as locations for NER factor recruitment (Zorn et al, 1979). Irradiation was carried out with a UVC source and energy of $150 \mathrm{~J} / \mathrm{m}^{2}$. Directly after irradiation the kept conditioned medium was pipetted into the wells $(500 \mu \mathrm{l}$ per well), the isopore membrane was removed and the different wells were incubated for the estimated timeline $\left[37^{\circ} \mathrm{C}, 5 \% \mathrm{CO}_{2}\right]$.

At the particular points in time, the cells were washed three times with PBS and fixed with $3.7 \%$ PFA supplemented with $0.1 \%$ Triton $\mathrm{X}-100$ or ice cold methanol (PCNA staining) for $15 \mathrm{~min}$ at room temperature. (At this stage an additional optional step can be performed. The appliance of a signal enhancer (life technologies) [diluted 1:1 in bidest, $200 \mu \mathrm{l} /$ well; 30min at RT], but this was not done during this thesis.) After washing, three times with PBS, a DNA denaturing step [20min, RT] follows. In case of DNA damages (6-4PP or CPD) $2 \mathrm{M} \mathrm{HCl}$ was used, $0.2 \mathrm{M} \mathrm{NaOH}$ during triple staining (see chapter 3.5.4). Next the samples were washed extensively with PBS (at least 5 times) and subsequently blocked with $10 \%$ FBS in PBS for $30 \mathrm{~min}$ at $37^{\circ} \mathrm{C}$ to avoid unspecific antibody binding. Further the cells were washed again with PBS (three times), the primary antibody was applied, as indicated (see Table 4), onto the cells and incubated $\left[30 \mathrm{~min}, 37^{\circ} \mathrm{C}\right]$. Followed by a $3 x$ washing step 
with PBS-Tween the secondary antibody, targeting an epitope of the primary antibody's species, was diluted as indicated (see Table 5) and disposed over the samples and incubated [ $30 \mathrm{~min}, 37^{\circ} \mathrm{C}$ ]. In case of 6-4PP staining a third "antibody" was used. The well established biotin-streptavidin signal enhancing mechanism was implemented. Herein, a biotinylated secondary antibody is recognized by a fluorescent label streptavidin molecule. After incubation with the labeled antibodies the cells were washed extensively (three times PBS-Tween, 2 times PBS) and subsequently mounted using an appropriate mounting medium. A clear nail polish serves as glue for fixing the glass cover slips onto the object slides. Pictures were taken using Axio M1 fluorescence microscope and the corresponding filter set to the fluorescent dye of the secondary antibodies (plus Axiovision software).

\subsubsection{Triplestaining - simultaneous detection of DNA damage, DNA repair synthesis and PCNA recruitment (Triple-IF)}

The Triplestaining (Triple-IF) is a combination of UDS (see chapter 3.5.2) and immunofluorescence (see chapter 3.5.3) and is, in this fashion presented here, conducted the first time ever. It allows simultaneous investigation of repair synthesis and recruitment of NER factors to sites of DNA damage in living cells.

After seeding cells (see chapter 3.3.1) prior to transfection (see chapter 3.3.4) an incubation of $48 \mathrm{hrs}$ $\left[37^{\circ} \mathrm{C}, 5 \% \mathrm{CO} 2\right]$ ensures proper (over-)expression of the relevant proteins. Before the procedure starts with the prefixing step of UDS (see chapter 3.5.2) the irradiation was carried out with $5 \mu \mathrm{m}$ isopore filters like it is done prior to immunofluorescence staining (see chapter 3.5.3). Additionally, the pre-fixed cells were washed twice with ethanol to ensure the removal of superfluid pseudoliposomal complexes (due to transfection) with absolute and $70 \%$ ethanol respectively for five minutes. Like UDS, cells were blocked with $10 \%$ FCS prior to the adding of the UDS reaction cocktail, but in contrast to the UDS, the terminal fixation step was carried out using ice cold methanol [20min, $\mathrm{RT}$ ], followed by a denaturing step using $0.2 \mathrm{M} \mathrm{NaOH}$ [20min;RT]. In the following the respective primary antibodies against DNA damages (CPD) and proteins (PCNA) are added together, as mixture of the specified dilutions (see Table 4), to the cells prior to the adding of the fluorescent secondary antibodies. All washing, incubation and visualizing steps are done like denoted in the immunofluorescence protocol (see chapter 3.5.3).

Analyzes was carried out using the Keyence microscope. Pictures of single cells were taken using the Z-stack (2-4 pictures) option independently in three channels. The wavelength of the channels was set according to the requirements of the secondary antibodies and UDS (see Table 5, see chapter 3.5.2). Using the analysis software (BZII-Analyzer), Z-stack pictures were merged to single pictures. Afterwards, haze reduction (sharpening) was performed (just brightness, automatic). Subsequently, 
the black balance option was used to blank the cytosolic background fluorescence (automatic, value always 128). This was performed separately for all three channels and the single pictures were overlayed to create a merge picture (see Figure 25). Red spots indicate CPD staining without UDS or PCNA recruitment. Yellow spots display a clear specific merge for CPD and UDS, which turns more into orange color if PCNA is also present. However, this should not be taken too serious, because it also can lead to misinterpretation because of the nuclear PCNA background staining.

\subsubsection{Post-UV cell survival estimation (MTT assay)}

Primary fibroblasts were seeded (see chapter 3.3.1) in a density of 3000 cells/well in $100 \mu$ l culture medium on a 96-well plate. On the next day, the medium of the wells was soaked and washed, and the rows of the 96-well plate were incubated with an increasing dose (ranging from 4 up to $180 \mathrm{~J} / \mathrm{m}^{2}$ ) UVC light. Subsequently, cells were incubated $\left[37^{\circ} \mathrm{C}, 5 \% \mathrm{CO}_{2}, 48 \mathrm{hrs}\right]$ with fresh DMEM. Afterwards, $15 \mu$ l dye-solution [MTT (3-(4,5-Dimethylthiazole-2-yl)-2,5-diphenyl-tetrazoliumbromid)- containing] was added to each well and the plates were again incubated $\left[37^{\circ} \mathrm{C}, 5 \% \mathrm{CO}_{2}, 4 \mathrm{hrs}\right]$. In this time the cells are metabolizing MTT by the action of mitochondrial dehydrogenases resulting in a blue colored formazan salt. After four hours the reaction is stopped by adding $100 \mu \mathrm{l}$ Stop-solution (dimethyl sulfoxide or sodium dodecyl sulfate diluted in hydrochloric acid) to each well and insoluble formazan is dissolved into a colored solution. Subsequently, the plate was incubated over night at room temperature to achieve cell lysis (light protected). The absorbance at $570 \mathrm{~nm}$ of each well was measured using the Tecan Elisa reader. According to the amount of formazan (metabolized MTT) the cell viability was estimated. The solutions from the colorimetric CellTiter96® Non-Radioactive Cell Proliferation Assay, provided by Promega, also called MTT-Assay, were used. 


\section{Results}

\subsection{Identification of a novel XPG patient and the corresponding mutant allele with consequences for the functional XPG-TFIIH interaction}

The University medical center Göttingen (UMG) collaborates with the university clinic Mannheim. Prof. Ernst G. Jung is a specialist for Xeroderma Pigmentosum (XP) (see chapter 1.1) patients and established a XP cell- and database from 1970 until his retirement in the year 2000. We, the department of Dermatology at the university clinic Göttingen (UMG), continued his research. We assumed the cell- and database with already established 100 cases from Mannheim and further expanded it to about 150 patients to date.

During the phenotype-genotype correlation studies I performed molecular and functional characterization of a patient with suspicion of severe XP/CS (see chapter 1.1.2). The 5-years-old Turkish girl (XP172MA) from a consanguineous family exhibits XP symptoms (e.g. freckling on UV exposed skin areas) as well as mental retardation, microcephaly, and cerebellar atrophy. We performed molecular-genetic analysis approved by our local ethics committee and according to Declaration of Helsinki principles.

Primary XP172MA skin fibroblasts revealed a severely reduced post UV-cell survival as assessed by the post-UV survival assay (see Figure 16) (see chapter 3.5.5). The $\mathrm{LD}_{50}$ value calculated was extraordinarily reduced $\left(4 \mathrm{~J} / \mathrm{m}^{2} \mathrm{UVC}\right)$ in patient's cells compared to wildtype fibroblasts $\left(175 \mathrm{~J} / \mathrm{m}^{2}\right)$, like it is characteristic for a serious XP disease.

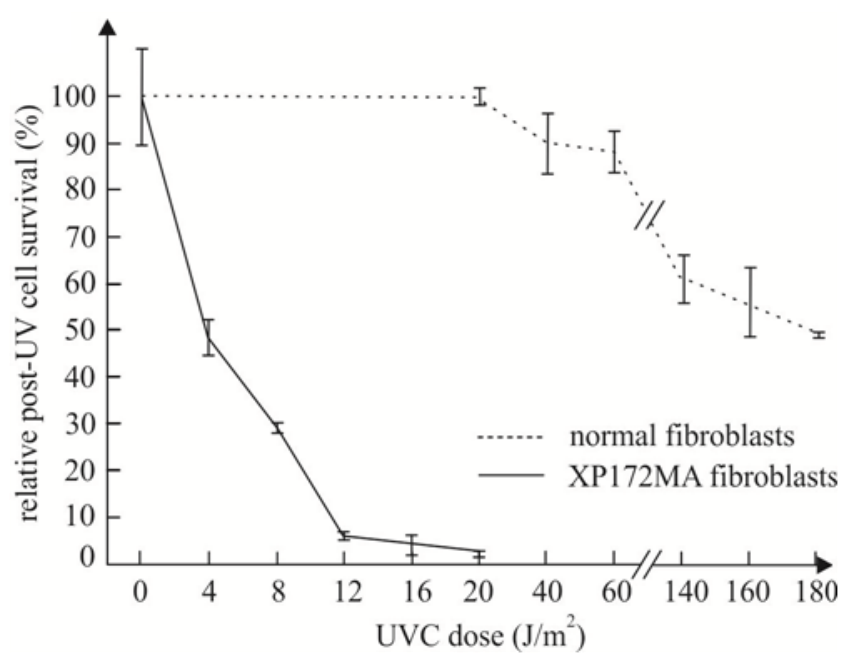

Figure 16: Post UV survival of XP172MA

The cell metabolism of patient's fibroblasts was highly reduced after exposure to UVC light in a doseincreasing manner $\left(0-180 \mathrm{~J} / \mathrm{m}^{2}\right) .12$ $\mathrm{J} / \mathrm{m}^{2}$ UVC light is sufficient to decrease cell viability of XP172MA by $90 \%$. In contrast the viability of wildtype cells was unaffected at this dose.

Further, the host cell reactivation (HCR) assay was used to determine the cellular NER capability (see chapter 3.5 .1 ). Wildtype fibroblasts have a relative NER capacity of about $16 \%$ while the patient cells 
exhibited a decreased repair capacity of only $0.1 \%$ (see Figure 17). Complementation using a wildtype XPG cDNA encoding expression vector for co-transfection resulted in an increased repair capacity of XP172MA cells of $10.5 \%$ close to normal repair level assigning the patient to the rare XP-G complementation group.

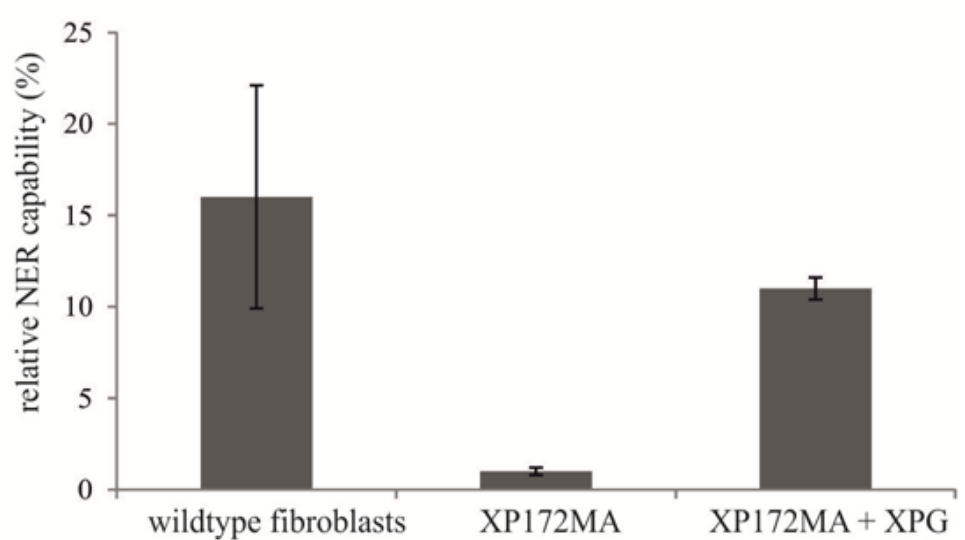

Figure 17: Assignment of XP172MA to XP Complementation group $\mathbf{G}$

Co-transfection of XP172MA cells with XPG cDNA expression vector increased the NER capability in a highly significant manner.

In contrast to other results in this work, the HCR assay was carried out in 6-well format by using the lipofectamine transfection agent (Qiagen).

Sequencing of chromosomal and complementary DNA (mRNA derived) (see chapters 3.2.3.1, 3.2.4.2) was performed to specify the disease-causing mutation responsible for XP172MA's XP/CS phenotype. It revealed an unusual homozygous genomic deletion of approx. $6.7 \mathrm{kbp}$ using g285for (ex1) and g870rev (ex6) primers (see Table 10). The deletion starts at the end of intron1-2 at position 7440 and ends in intron5-6 at position 14166, thereby retaining nine bases of intron5-6 (positions 141 4414152) (according to GenBank accession number NM.000123.2).

Additional sequencing of the XPG CDNA, with the same primers mentioned above, identified two variants, resulting from the deletion of the exons 2-5 (see Figure 18) (see chapter 3.2.4.2). Both splicevariants comprise a deletion between exons one and five. Furthermore the PCR (see chapter 3.2.3.2) with patient's cDNA as template revealed a band approx. $450 \mathrm{bp}$ shorter than wt, consistent with the sequencing result (data not shown). 


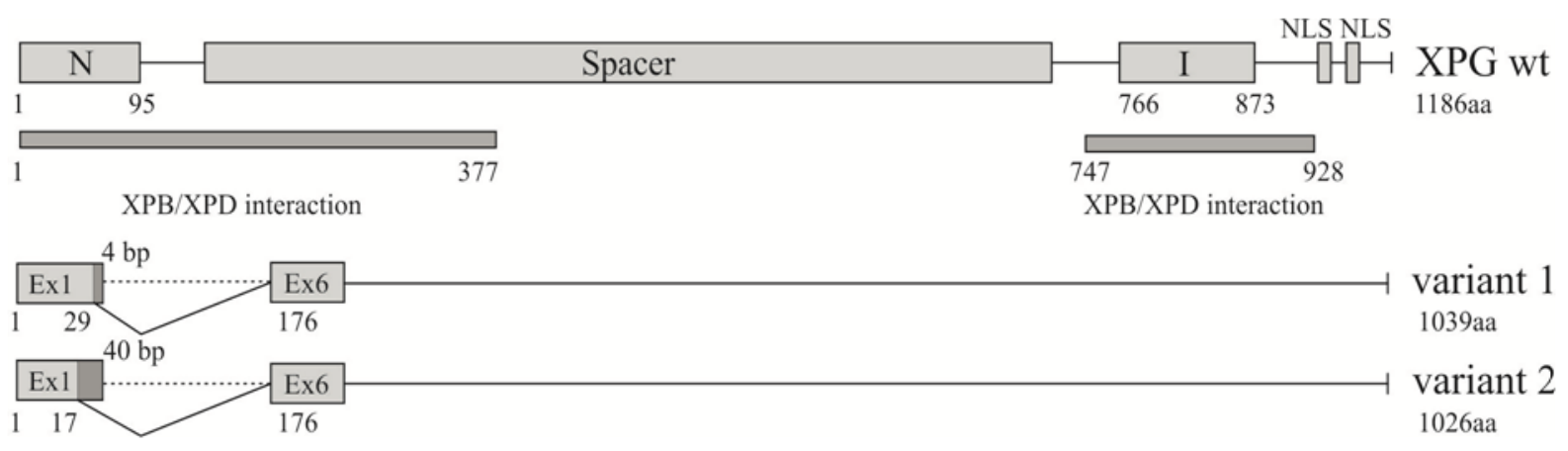

\section{Figure 18: XP172MA alleles}

Variant one consisted of an in-frame deletion of the last four bases of exon 1 and the entire exons 25. The predicted protein is truncated missing aa29-175. In variant two 36 more basepairs of exon 1 were deleted together with exons 2-5 also leading to a truncated in frame XPG protein lacking aa17176.

Several further arguments underline the correct identification of the disease-causing mutation in the XP172MA patient. Using Qiagen QuantiTect Primer Assays (annealing sequence in the N-terminus of $X P G)$, designed to prime in the deleted stretch, zero mRNA expression was detected due to the missing template in the cDNA of the patient (data not shown). Furthermore protein expression of the longer variant was observed by western blotting, utilizing an anti-XPG antibody utilizing (Lehmann, 2013) (see chapter 3.4.4). Janin Lehmann furthermore displayed in her master thesis (under my supervision) the nuclear localization of the GFP-tagged recombinant shorter XP172MA protein. Both findings clarify, that the corresponding protein is expressed in a stable fashion and does not underlie enhanced proteosomal digestion. Hence, the identified huge in frame chromosomal deletion is causes the XP/CS complex phenotype of patient XP172MA due to the non-functionality of the mutated XPG protein during NER. The combined XP/CS phenotype can be explained by missfunction of the XPG protein during transcription, especially displaying impaired TFIIH interaction (see chapter 1.4).

This shows the importance of genotype-phenotype correlation studies. Physiologically occurring XPG variants in humans demonstrate the clinical effects. Due to the patient XP172MA alleles, I have to narrow down the functional XPG-TFIIH interaction region (see chapter 5.2). These results will soon be available in the British journal of Dermatology (under revision).

\subsection{The functional interaction between PCNA and XPG is essential during NER}

The investigated XPG protein is multifunctional in several cellular pathways (see chapter 1.4). Furthermore, it administrates a lot of interactions with several proteins, e.g. PCNA (see chapter 1.3.5). To shed a light on the functionality and interactions of XPG, I created, amongst others, several PCNA interaction compromising site specific mutants of XPG, including an ubiquitin interaction (see Table 14, see Figure 19) (see chapters 4.2.1, 3.2.3.4). 


\subsubsection{XPG mutants created and used during this study}

The XPG mutants created and used during this project as well as the corresponding domains of the proteins are marked in Figure 19. Quickchange mutagenesis was performed to create the in vitro endonuclease defective mutants $E 791 A$ and $D 77 A$ as well as the corresponding double mutant and E791A-FF (DM_E/FF) mutant. The PCNA interaction defective mutants FF and Del were a gift from Prof. Ronald Gary and subcloned into the same vector (pcDNA3.1 (+)), used for cloning of all XPG mutants (see chapter 3.2.3.4). Fen1 and D181A were purchased from addgene.org, a good expression level was detected using western blotting (data not shown). DNA2 and IsoVI were cloned from human cDNA with specific primers, IsoVI by Janin Lehmann under my supervision (Lehmann, 2013). PIP and UBM single, double, and triple mutants were also mutated via quick change mutagenesis (see chapter 3.2.3.3). Table 14 and Figure 19 illustrate these features with regard to the consequences on protein level.

Table 14: Recombinant mutants of XPG and other enzymes used during this thesis

\begin{tabular}{|c|c|c|}
\hline Nature & Description & Defect \\
\hline \multirow[t]{7}{*}{ Full lenght XPG } & E791A, D77A & Endonuclease defective XPG (Constantinou et al, 1999) \\
\hline & DM_E/D & Endonuclease defective double mutant E791A \& D77A \\
\hline & $\mathrm{FF}$ & PIP-box mutated XPG F996A and F997A (Gary et al, 1997) \\
\hline & DM_E/FF & $\begin{array}{l}\text { Endonuclease defective }+ \text { PIP-box mutated double mutant } \\
\text { E791A, FF }\end{array}$ \\
\hline & UBM & $\begin{array}{l}\text { Ubiquitin Binding defective XPG, L196A and P197A } \\
\text { (Fagbemi, 2012) }\end{array}$ \\
\hline & PIP & $\begin{array}{l}\text { PCNA binding compromised XPG, PIP-N domain, P179A } \\
\text { (Fagbemi, 2012) }\end{array}$ \\
\hline & UBM-PIP & $\frac{\text { Ubiquitin Binding defective }}{\text { double mutant UBM, PIP-N }}+\underline{\text { PCNA binding compromised }}$ \\
\hline \multirow[t]{2}{*}{ Truncated XPG } & Isoform VI (IsoVI) & $\begin{array}{l}\text { physiological relevant XPG splicevariant, 746aa (Lehmann, } \\
\text { 2013) }\end{array}$ \\
\hline & Del & artifically truncated XPG, 948aa (Gary et al, 1997) \\
\hline \multirow[t]{3}{*}{ Other nucleases } & Fen1 & Flap endonuclease 1, $42.5 \mathrm{kDa}$ (380 aa) \\
\hline & D181A & Endonuclease defective Fen1 \\
\hline & DNA2 & Nuclease/Helicase, 45 kDa \\
\hline
\end{tabular}




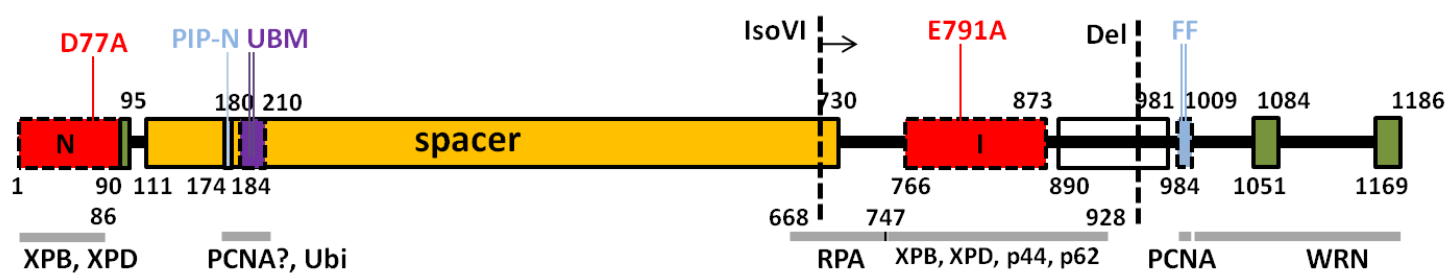

Figure 19: Mutated residues of XPG

The names of mutants used during this study are depicted above, vertical lines indicate for the mutation sites, the dotted lines show sites of truncations (IsoVI additionally comprises 11 intronic residues depicted by the black arrow to the right) (references in text, see chapter 1.4.2).

The expression of several mutants was exemplarily monitored on protein level using western blotting (see Figure 20) (see chapter 3.4.4). The test comprises wildtype XPG (WtXPG), ubiquitin binding defective (XPG-UBM), in all probability PCNA and ubiquitin binding defective (XPG-PIP-UBM), truncated variants of XPG (IsoVI, Del), as well as endonuclease defective XPG (E791A, D77A).

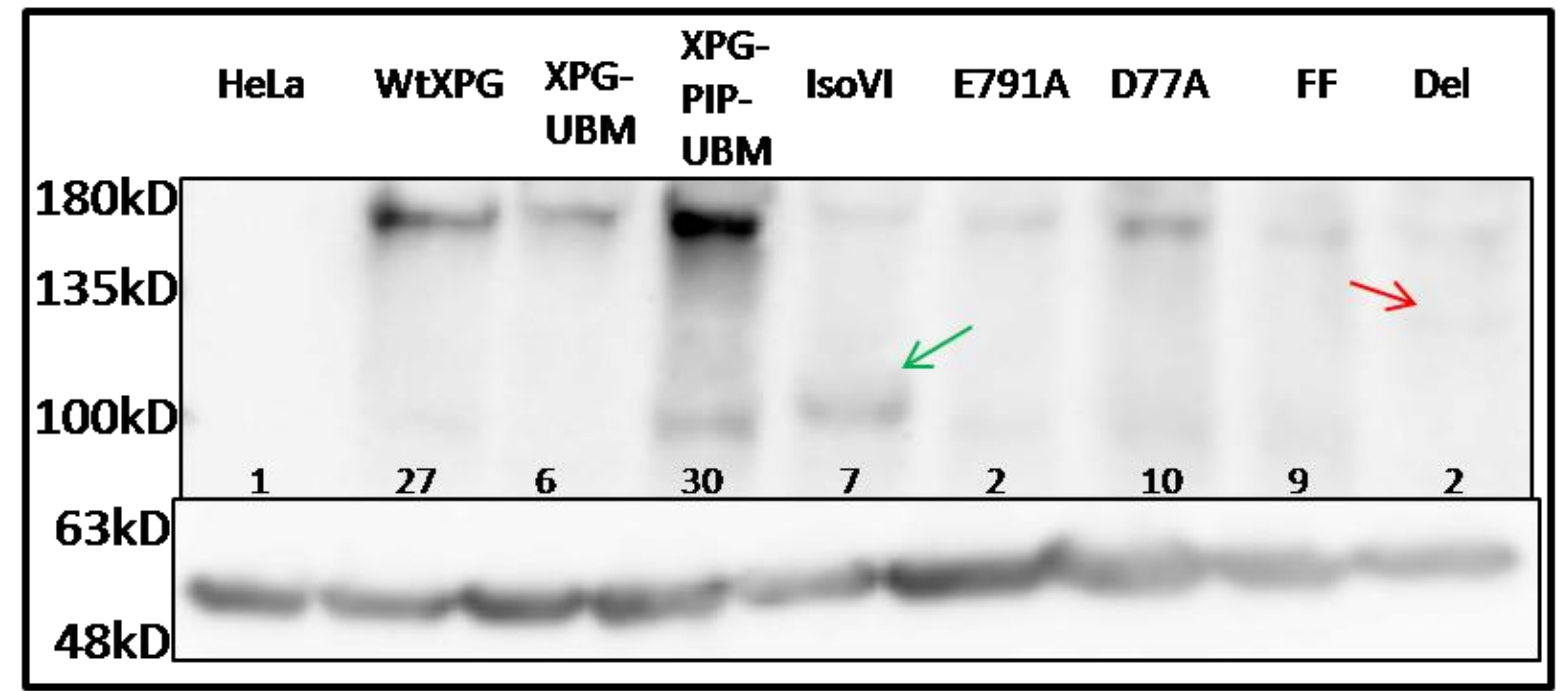

Figure 20: Overexpression of XPG mutants in HeLa cells

$48 \mathrm{~h}$ hours after transfection the cells were lysed and the proteins were harvested, electrophoretically analyzed and blotted for immunochemical analysis (see chapter 3.4.4). The XPG blot is depicted on top; $\beta$-actin for normalization is displayed on the bottom. The small black numbers indicate the $x$-fold expression enhancement after normalization in comparison to untransfected HeLa cells. XPG, has a molecular mass of $133 \mathrm{kDa}$, but migrates at a size of 180 kDa due to its highly acidic patches (isoelectric change). The $\beta$-actin protein has a size of $47 \mathrm{kDa}$. Both truncated mutants also migrate higher than their predicted molecular mass. Green arrow $=$ IsoVI (MW: $\sim 22,5 \mathrm{kDa})$, red arrow = Del $(\mathrm{MW} \sim 100 \mathrm{kDa})$.

All cloned variants are overexpressed in the desired fashion in HeLa cells, quite differently, from 2 up to 30 fold compared to wildtype, whereas the poorly expressed endogen XPG of the HeLa control is not detectable at all (see Figure 20). The estimated expression level methodically depends on several factors (transfection efficacy, protein preparation efficacy, blotting efficacy) and the differences of the respective mutants will not be further discussed. The experiments were done in numerous 
repeating (HCR) or with a high number of analyzed cells (UDS) to minimize the influence of methodically unavoidable differences (see chapters 3.5.1, 3.5.2).

\subsubsection{Immediate Unscheduled DNA repair synthesis (UDS) of XPG mutants}

In order to investigate the responsibility and ability of XPG to recruit PCNA and subsequently start DNA repair synthesis (UDS, see chapter 3.5.2), several XPG mutants were created and transiently transfected into XP2OBE cells (see chapters 3.3.4, 4.2.1). The UDS assay was performed two hours after UV irradiation of these (probably) complemented cells and the amount of DNA synthesis within this short time was quantified. The depicted UDS levels in Figure 21 display the immediate repair rate in dependency of the XPG-mutants.

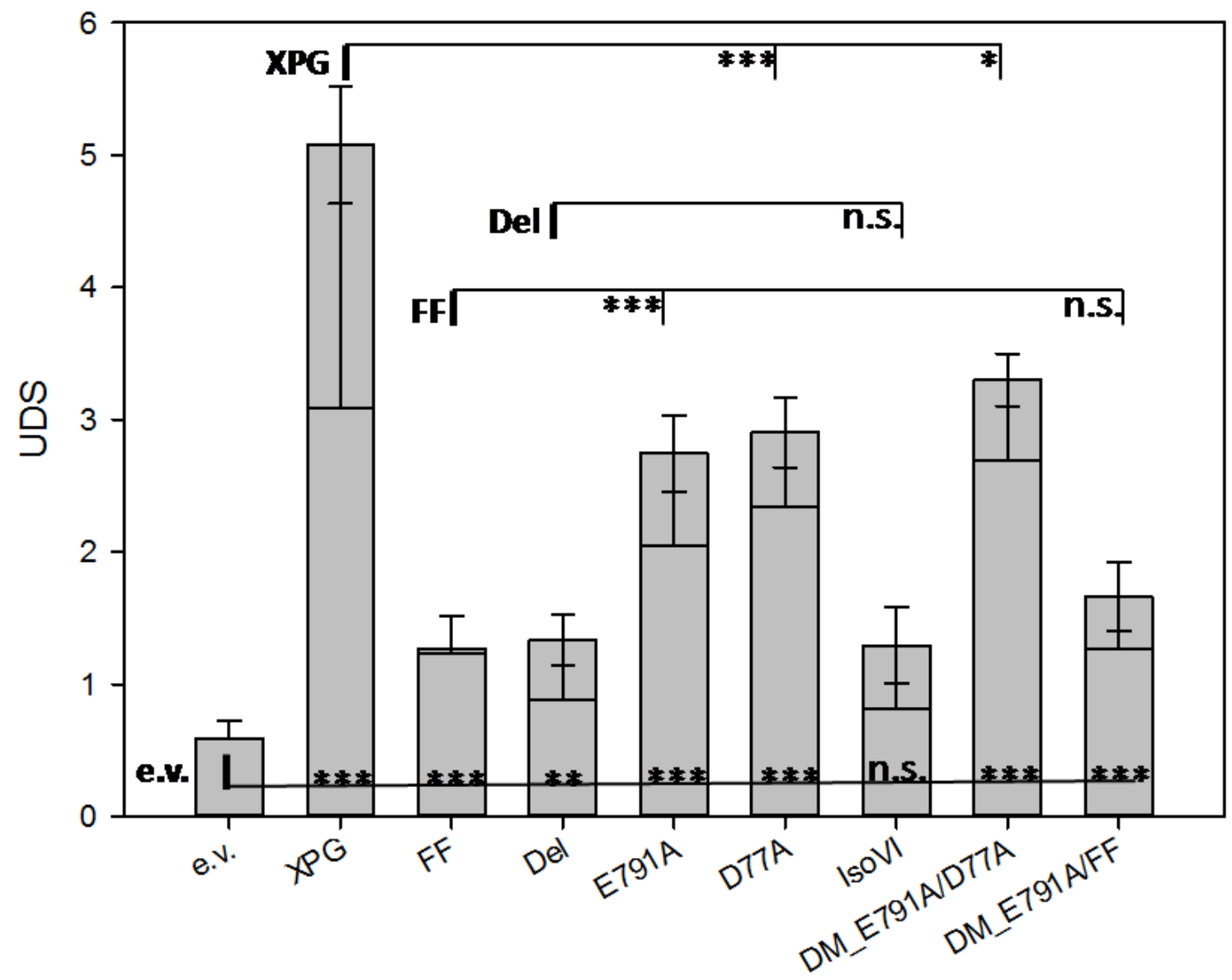

Figure 21: UDS results of XPG mutants

The grey bars represent the mean values of UDS (see chapter 3.5.2) of XP20BE nuclei two hours after irradiation. The cells were transiently transfected with several XPG mutants (see chapter 4.2.1). The small black horizontal lines of each bar indicate the median value. Significance levels were correlated using the Mann-Whitney U-Test for abnormally distributed series of measurements $\left({ }^{* * *}=p<0.001\right.$, ${ }^{* *}=p<0.01,{ }^{*}=p<0.05$, n.s. $=$ not significant; e.v. $=$ empty vector; $\mathrm{DM}=$ double mutant). The small fat vertical lines, in the left of each significance comparison, mark the reference group (see also Table 14, Figure 19). 
All analyzed XPG mutants display a significantly enhanced UDS level compared to the empty vector negative control (Del: $\mathrm{p}<0.01$, all others: $\mathrm{p}<0.001$; Mann-Whitney-U-test). IsoVI, a preselected isoform of XPG with NER capacity (using HCR, see chapter 3.5.1), is the exception in this case, with a non-significant outcome (Lehmann, 2013). However, a control of diluted IsoVI 1:2 with empty vector displayed a significant enhancement ( $p<0.001$; Mann-Whitney-U-test) compared to the empty vector (see Figure 26). This fact probably displays the problems of cells due to overexpression of a recombinant protein.

The comparison of the UDS median values after complementation in most of the cases shows a highly significant decrease of all mutants compared to the wildtype XPG positive control (DM_E/D: $p<0.05$, all others: $p<0.001$; Mann-Whitney-U-test). The wildtype XPG itself transferred about $40-50 \%$ activity to XP20BE cells, compared to WT fibroblast of a healthy donor (see Figure 26). However, this is in a range like it was expected with a transfection efficacy of about $50 \%$ (data not shown).

All endonuclease defective mutants show a highly significant increase $(p<0.001$; Mann-Whitney-Utest) compared to the XPG FF mutant (see Figure 21), which in turn is nonsignificantly different compared to the truncated XPG variants (Del, IsoVI) (Mann-Whitney-U-test). The double mutant FFE791A indicates no significant difference compared to the PIP-box FF single mutant (Mann-WhitneyU-test).

Both truncated proteins lack the I-domain (endonuclease) as well as the C-terminal canonical PIP-box (see chapter 4.2.1). Both XPG variants complement the UDS assay on the same level. IsoVI is not significantly enhanced compared to the empty vector (Mann-Whitney-U-test), whereas Del complements with the lowest significance of all complementations ( $p<0.01$; Mann-Whitney-U-test).

Taken together, the immediate DNA repair synthesis rate is mainly dependent on an intact PIP-box. Endonuclease defective mutants also show a decreased UDS rate compared to wildtype, nevertheless, UDS is still remarkably increased compared to PIP-Mutants or truncated XPG proteins ( $p<0.001 ;$ Mann-Whitney-U-test).

To investigate the influence of the N-terminally located PCNA interaction domains, PIP-N and UBM, single, double, and triple mutants on the basis of wt-XPG, FF, E791A, and IsoVI were created (see chapters 3.2.3.3, 4.2.1, Figure 19). XPG defective XP20BE cells were transiently transfected (see chapter 3.3.4) with the mutant XPG cDNA expression vectors and analyzed regarding the immediate DNA repair synthesis response utilizing UDS (see Figure 22). 

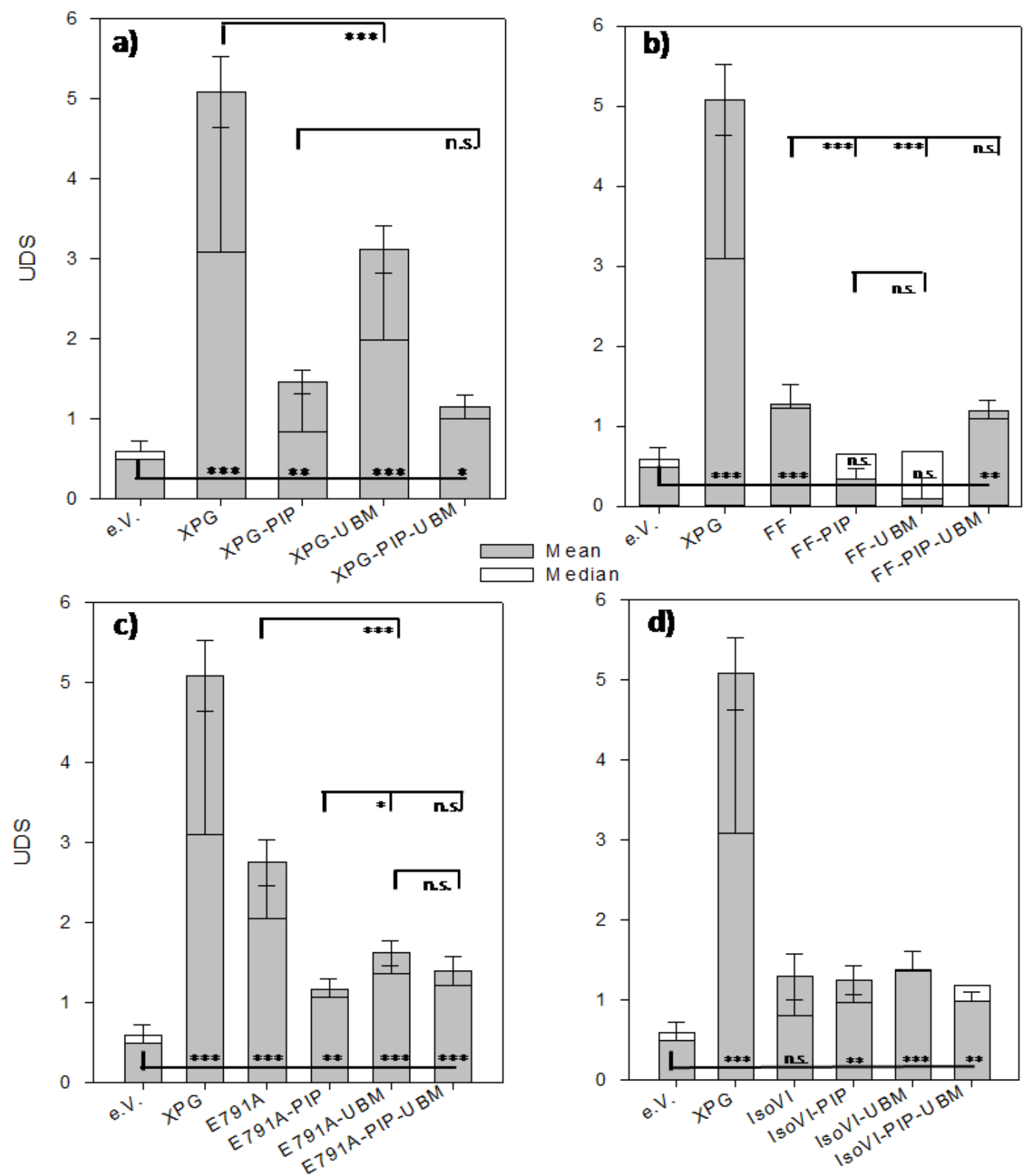

Figure 22: UDS results of PIP-N and UBM mutants

A) XPG b) FF c) E791A and d) IsoVI were used as template for additional mutagenesis of the PIP-N and UBM domain of XPG (see chapter 4.2.1). The small black horizontal lines or the white box of each bar indicate the median value of UDS two hours after irradiation. Significance levels were correlated using the Mann-Whitney U-Test for abnormally distributed series of measurements $\left({ }^{* * *}=p<0.001\right.$, $*^{* *}=p<0.01,{ }^{*}=p<0.05$, n.s. $=$ not significant; e.v. = empty vector). The small fat vertical lines, in the left of each significance comparison, mark the reference group.

Compared to wt XPG the single PIP-N mutation alone (see chapter 4.2.1) was sufficient to reduce the immediate UDS (see chapter 3.5.2) rate, measured two hours after UVC irradiation, in a highly significant manner ( $p<0.001$; Mann-Whitney-U-test) (see Figure 22a). The XPG-UBM mutant showed also a significant decrease compared to wt, but in turn highly significant increase compared to XPGPIP ( $p<0.001$; Mann-Whitney-U-test). The double mutant XPG-PIP-UBM behaved like the XPG-PIP 
single mutant. The PIP mutant showed a comparable low UDS level to the FF-mutation alone (not significant, Mann-Whitney-U-test), designating the residue P179 to a new essential factor for immediate UV irradiation response (see Table 14, see chapter 4.2.1).

The postulated PCNA-interaction defective mutant $F F$ was additionally mutated to the FF-PIP and FFUBM double as well as FF-PIP-UBM triple mutants (see Figure 22b). The UDS levels of both double mutants showed an even more impaired repair synthesis rate compared to the FF-mutation alone ( $p<0.001 ;$ Mann-Whitney-U-test). Surprisingly, the developed triple mutant displayed synthesis activity on the same level of the $F F$ mutant alone.

The addition of PIP- and/or UBM- mutations to the E791A mutant combines an endonuclease defect with the disturbance of interactions between PCNA or ubiquitin and XPG, respectively (see Figure $22 c)$. All mutants, whether double or triple, show a highly significant $(p<0.001$; Mann-Whitney-Utest) decrease of UDS in comparison to the E791A mutation alone but significantly enhanced with the empty vector as the control group (E791A: $p<0.01$, all others: $\mathrm{p}<0.001$; Mann-Whitney-U-test). The UDS rate in dependency of E791A-UBM is slightly significant increased compared to the E791A-PIP mutant ( $p<0.05$; Mann-Whitney-U-test). Both PIP- as well as the UBM-mutation containing endonuclease defective mutants display a low DNA synthesis on FF level (no significant difference; Mann-Whitney-U-test).

A really unexpected picture was displayed using PIP- and/or UBM single- or double mutants of the XPG Isoform VI depicted in Figure 22d. The splice variant IsoVI (see chapter 4.2.1) was selected during the master thesis of Janin Lehmann under my supervision with regard to the highest XP20BE complementation ability using Host Cell Reactivation (also see Figure 23) (Lehmann, 2013).

The isoform itself displays only a weak, non significant increase compared to the empty vector control in this figure (Mann-Whitney-U-test). However, as mentioned before a control diluted with empty vector displayed a significant enhancement compared to the empty vector, in contrast to IsoVI alone (see Figure 26).

Both mutations PIP and UBM as well as the double mutant shift the increase of UDS rate into a (partly highly) significant area (IsoVI-UBM: $p<0.001$, all others: $p<0.01$; Mann-Whitney-U-test). The statistical comparison between IsoVI and the particular PIP- and UBM mutants displayed no significant difference. Hence, all IsoVI mutants display about the same rate of UDS and are able to facilitate a reduced but sill significant DNA synthesis after UV irradiation on the same level like the FF mutant. 
Taken together these results indicate an essential role of the N-terminally located PIP-N and UBM boxes of XPG. Due to the fact that there is no dramatic difference between the UBM and PIP mutations and as cumulative reducing effect of both mutations in concert was observed, it is obvious that these two domains act in concert. The significant UDS increase of the UBM mutant compared to PIP mutant in wt-XPG and E791A proteins possibly indicated for a more important support of the functional main PCNA recruiting domain (C-terminal PIP box) rather by the PIP-box than by the UBM box. The unexpected increase of UDS due to the FF-PIP-UBM triple mutant compared to the FF-PIP and FF-UBM mutants will be discussed later (see chapters 4.2.4, 5). The weak but detectable complementation ability of IsoVI utilizing UDS was, in contrast to all other XPG species, not further compromised by PIP or UBM mutations. The simplest solution to explain this fact is that it behaves in a different fashion than the normal XPG.

\subsubsection{Host cell reactivation assessment of XPG mutants}

Utilizing the UDS (see chapter 3.5.2) assay, I was able to investigate the immediate DNA synthesis after irradiation in dependency of multiple XPG mutants. However, I further analyzed how these mutants complement the XPG deficiency in XP20BE fibroblast cells via transient overexpression and performance of the host cell reactivation (HCR) assay three days after transfection (see Figure 23) (see chapter 3.5.1). 


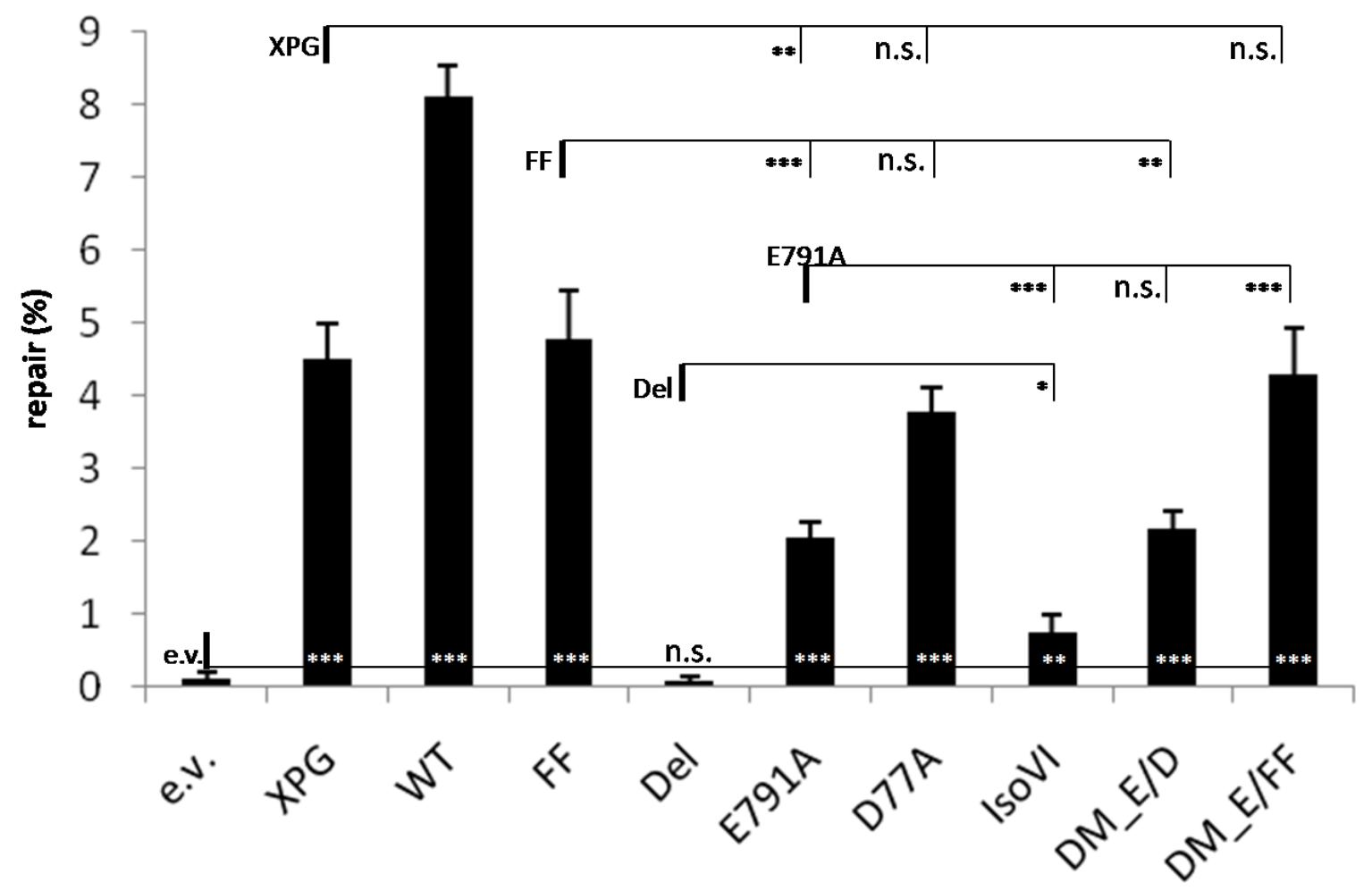

Figure 23: Host cell reactivation of XP20BE cells by XPG mutants

The black bars indicate the relative NER capacity of an UV irradiated Firefly reportergene plasmid compared to the unirradiated expression level (normalized to the cotransfected Renilla luciferase, see chapter 3.5.1) in dependency of various XPG mutants, three days after transfection. The error bars are calculated using error propagation of the mean values of normalized Firefly, either irradiated or not, due to statistical standard techniques. Significance levels are calculated using the Student's TTest (comparison of two groups). The small fat vertical lines, in the left of each significance comparison, mark the reference group. Abbreviations: ${ }^{* * *}=p<0.001,{ }^{* *}=p<0.01,{ }^{*}=p<0.05$, n.s. $=$ not significant; e.v. $=$ empty vector; $\mathrm{WT}=$ healthy donor; $\mathrm{DM}=$ double mutant).

XP20BE cells transfected with the empty vector showed no complementation ability at all, whereas the transfection of wildtype XPG complemented this defect, measured three days after transfection. Wildtype cells of a healthy donor displayed an even more increased NER capacity, which is due to the fact that recombinant XPG in cured XP20BE cells, in contrast to endogen XPG in wt cells, first has to be expressed and modified etc. to a certain extent before it is able perform NER. Furthermore, the transient transfection approach supplies 50-60\% transfection efficacy (data not shown) (see chapter 3.3.4).

All full-length mutants ( $F F, E 791 A, D 77 A$ and their respective double mutants) increase the repair rate of the XPG deficient fibroblasts excessively in a highly significant manner compared to the empty vector ( $p<0.001$; Student's T-test). The truncated Isoform VI, preselected for NER activity, confirms that strong enhancement compared to the empty vector control ( $p=0.002$; Student's T-test). In contrast the artificially truncated XPG version Del is not able to complement. 
Both FF-mutation containing clones were able to complement XP20BE cells to the same extent compared to wildtype XPG. Furthermore the D77A endonuclease defective mutant (mutation in $\mathrm{N}$ domain) displayed also no significant difference. In contrast, the E791A mutation in the I-domain of XPG reduces the NER capacity compared to wt XPG, whether alone or in combination with D77A (E791A: $p<0.01$, DM_E/D: $p=0.06$; Student's T-test). However, due to the relatively low sample size in the case of $D M_{-} E / D$ ( $p$-value 0.006$)$, significance was only achieved in the case of $E 791 A$ alone. The fact that the DM_E791A/FF double mutant behaves like wt XPG indicates the cure of E791A-caused impaired NER by the lack of the C-terminally located PIP-box. This accounts possibly for another factor, which is favorable able to recruit PCNA and to perform endonuclease cleavage.

The endonuclease defective mutant $E 791 A$ itself in turn revealed an increased NER activity compared to the truncated versions Del and IsoVI ( $<<0.001$; Student's T-test). IsoVI represents the weakest XPG variant, but is significantly enhanced capable of performing NER compared to $\mathrm{Del}(\mathrm{p}<0.05$; Student's T-test).

Taken together, these results indicate a strong influence on NER capability by the essential E791 mutation to alanine and a detectable but significantly decreased activity of Isoform VI. The next figure displays the HCR analysis of several XPG PIP- and UBM mutants (see Figure 24, see chapter 4.2.1). 
a)
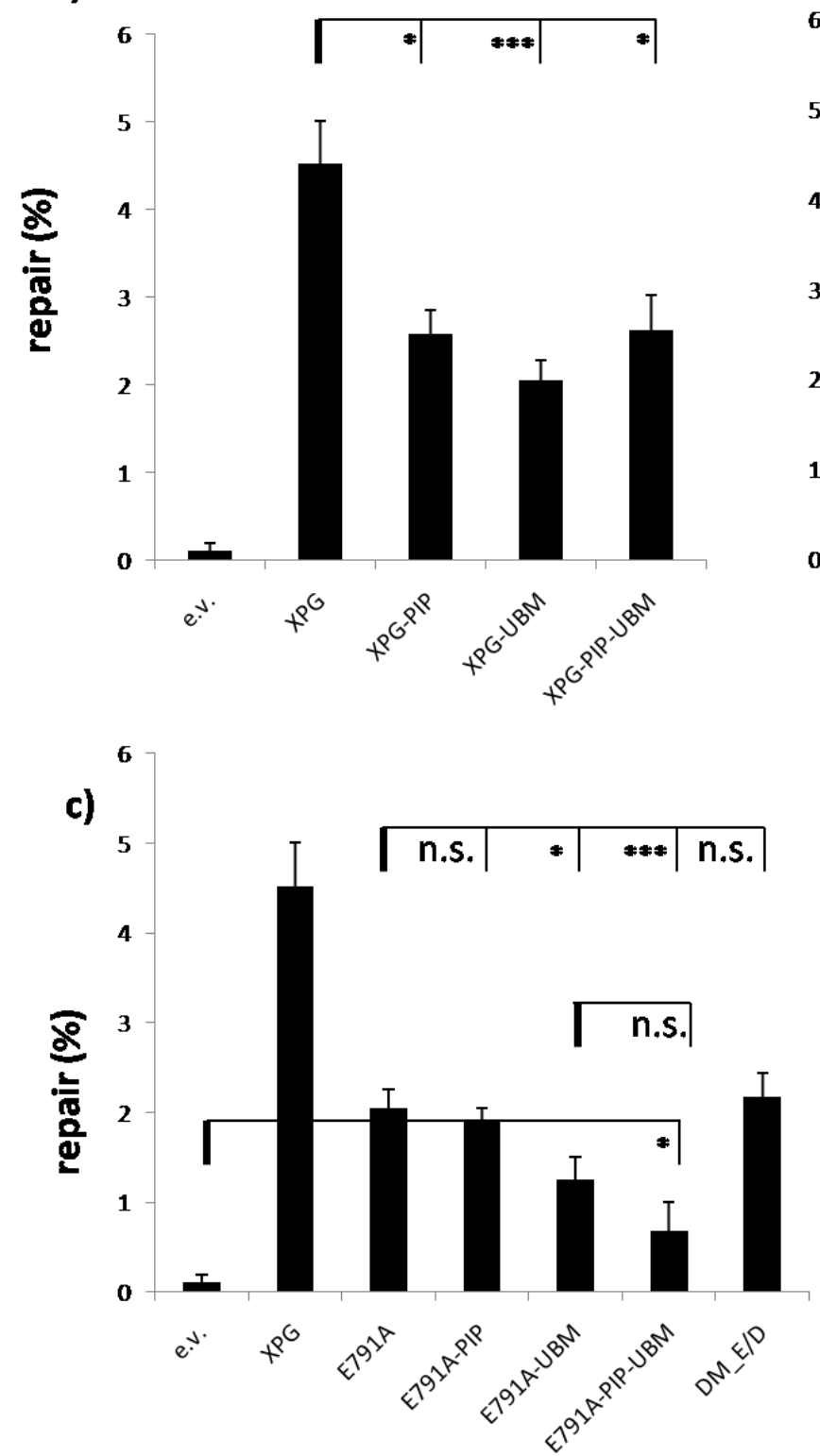

b)
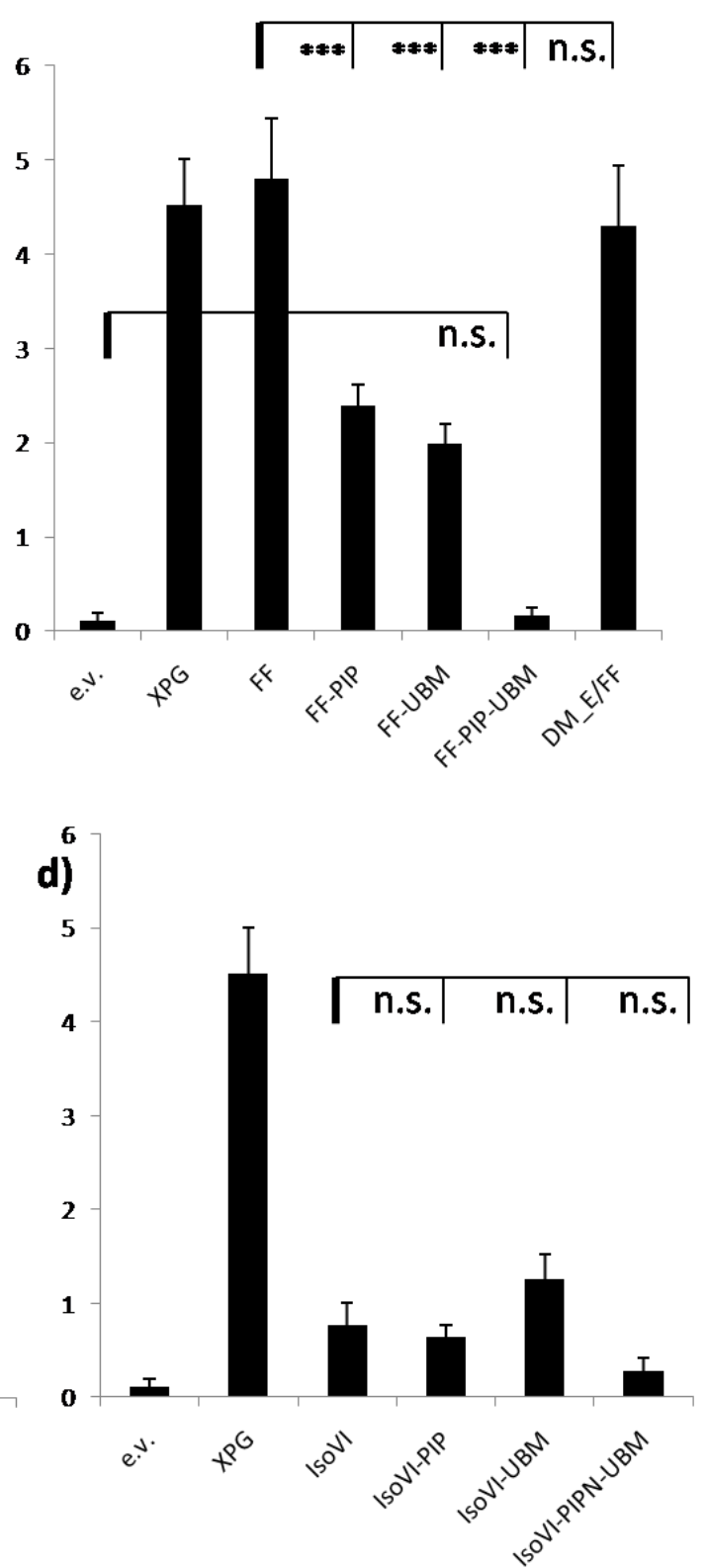

Figure 24: HCR of PIP- and UBM mutants as well as the respective double- and triple mutants A) XPG b) FF c) E791A and d) IsoVI were used as template for additional mutagenesis of the PIP-N and UBM domain of XPG (see chapter 4.2.1). The black bars indicate the relative NER capacity of an UV irradiated Firefly Reportergene plasmid compared to the unirradiated expression level (normalized to the cotransfected Renilla luciferase, see chapter 3.5.1) in dependency of various XPG mutants, three days after transfection. The error bars are calculated using error propagation of the mean values of normalized Firefly, either irradiated or not, due to statistical standard techniques. Significance levels are calculated using the Student's T-Test (comparison of two groups). The small fat vertical lines, in the left of each significance comparison, mark the reference group. Abbreviations: ${ }^{* * *}=p<0.001$, ${ }^{* *}=p<0.01,{ }^{*}=p<0.05$, n.s. = not significant; e.v. = empty vector; $W T=$ healthy donor).

Both, the PIP- and UBM mutation, decrease the XP20BE complementation ability of wt XPG (see Figure 24a) in a significant manner (XPG-UBM: $p<0.001$, all other: $p<0.05$; Student's T-test). The displayed difference of reduced significance levels of all PIP mutants compared to the UBM mutant, are due to the relative low sample size of HCR measurements of the PIPs compared to all others. 
Methodical difficulties during mutagenesis of this specific region lead only to the successful creation at the very end of the thesis. The PIP-, UBM-, and the respective double mutants all behaved in the same fashion like the endonuclease defective E791A mutant (no significant difference, Student's Ttest).

In comparison to the PIP-box mutated FF mutant (see Figure 24b), all further PCNA interaction impairing mutations, PIP- and UBM, particularly decrease the complementation activity of the FF clone to a very significant extent in the double mutants ( $p<0.001$; Student's T-test). Most strikingly, the triple mutant FF-PIP-UBM is not able to perform NER, whereas the respective single mutants complement on E791A level.

The E791A mutation was combined with PIP- and UBM mutagenesis and also analyzed utilizing HCR (see Figure 24c). The PIP mutation had no influence on the behavior, indicated by the E791A-PIP double mutant compared to E791A alone. Though the E791A-UBM mutant and the triple mutant E791A-UBM-PIP decrease the observed NER capability in a weak and strongly significant manner (E791A-UBM: p<0.05, E791A-UBM-PIP: p<0.001; Student's T-test), respectively. However, the E791PIP-UBM triple mutant shows no significant difference to the E791A-UBM or FF-PIP-UBM mutants, and behaved like the empty vector control (Student's T-test).

The XPG splice variant Isoform VI was also mutated via quick change mutagenesis in order to create single- as well as double mutants and analyzed with the HCR assay (Figure 24d). The performed mutagenesis displayed no significant difference of the respective mutants compared to Isoform $\mathrm{VI}$ (Mann-Whitney-U-test). However, the PIP- and UBM single mutants still statistically complement the XP20BE XPG deficiency (IsoVI-PIP: $p<0.01$, IsoVI-UBM: $p<0.001$; Student's T-test) in contrast to the corresponding double mutant IsoVI-PIP-UBM which is not significantly different from the empty vector control (Student's T-test).

Taken together, these results indicate that FF sites are dispensable for complementation, but not for immediate DNA repair synthesis (see chapter 4.2.4). The mutagenesis of PIP-N and UBM confers complementation deficiency to XPG on the same level like E791A mutation. The simultaneous mutagenesis of three sites (FF-PIP-UBM, E791A-PIP-UBM) leads to the prevention or strong restriction of NER activity. Additionally, the N-terminal mutations of PIP-N and UBM display a synergistic effect, which leads to the suggestion that they act in concert. 


\subsubsection{Short summary of the quantitative results}

Table 15: Lineup of XPG mutants

$X P G$ mutants are lined up regarding their classification of NER activities during the Host cell reactivation assay in comparison to the Unscheduled DNA synthesis measurements.

\begin{tabular}{|l|c|c|c|}
\hline & HCR & UDS & consistent \\
\hline Leer & $\downarrow$ & $\downarrow$ & yes \\
\hline XPG & $\uparrow$ & $\uparrow$ & yes \\
\hline FF & $\uparrow$ & $\searrow$ & no \\
\hline Del & $\downarrow$ & $\searrow$ & yes \\
\hline E791A & $\rightarrow$ & $\rightarrow$ & yes \\
\hline DM_E/FF & $\uparrow$ & $\downarrow$ & no \\
\hline FF-PIP & $\rightarrow$ & $\downarrow$ & no \\
\hline FF-UBM & $\rightarrow$ & $\searrow$ & no \\
\hline FF-PIP-UBM & $\downarrow$ & $\searrow$ & no \\
\hline E791A-PIP & $\rightarrow$ & $\searrow$ & yes \\
\hline E791A-UBM & $\searrow$ & $\searrow$ & yes \\
\hline E791A-PIP-UBM & $\searrow$ & $\searrow$ & yes \\
\hline IsoVI (all mutants) & $\searrow$ & & \\
\hline
\end{tabular}

In presence of wt XPG, the empty vector, isoform $\mathrm{VI}$ as well as most of the endonuclease deficient E791A mutants (except E791A-PIP) the HCR (see chapter 4.2.3) and UDS (see chapter 4.2.2) results show a similar picture (see Table 15). In these cases, the complementation ability was conferred or not regarding both activities: the immediate start of DNA repair synthesis and the termination of NER (accurate TCR) using either UDS or HCR, respectively (see chapters 1.3, 3.5.2, 3.5.1).

All FF mutants (except FF-UBM-PIP) as well as E791A-PIP mutant display an inconsistent picture with a good complementation ability (HCR) but a compromised immediate start of DNA repair synthesis (UDS) (see chapter 4.2.1). The notion, that the E791A-PIP mutant is the only endonuclease defective mutant with a similar result, indicates the importance of the functional XPG - PCNA interaction for DNA repair synthesis.

Furthermore, there is a decreased rate of NER activity (HCR) observed with rising numbers of interaction impairments (number of mutations) among the FF and E791A mutants. However, due to the fact that the FF-PIP-UBM mutant, comprising the highest number of PCNA-interactions impairing mutations including an ubiquitin interaction, showed a diverse picture with high UDS and low 
complementation ability, I raise a model which involves the obligatory ubiquitination of PCNA and the recruitment of translesion polymerases.

\subsubsection{Estimation of simultaneous PCNA-recruitment and UDS synthesis at DNA damage sites in dependency of XPG mutants}

There is a discrepancy between immediate DNA repair synthesis on the one hand (UDS) and the TCR complementation ability (HCR) on the other hand by the FF clone and its respective mutants (see Table 15) (see chapters 1.3.4.4, 3.5.2, and 3.5.1). I investigated the PCNA recruitment to DNA damage and UDS in that spot separately at the same time. This was performed to exclude the possibility of PCNA recruitment but a stalled synthesis machinery due to the XPG defect. Figure 25 shows Triple- Immunofluorescence staining (see chapter 3.5.4), in order to clarify the responsible interactions for PCNA interaction and/or start of synthesis in dependency of XPG and FF.
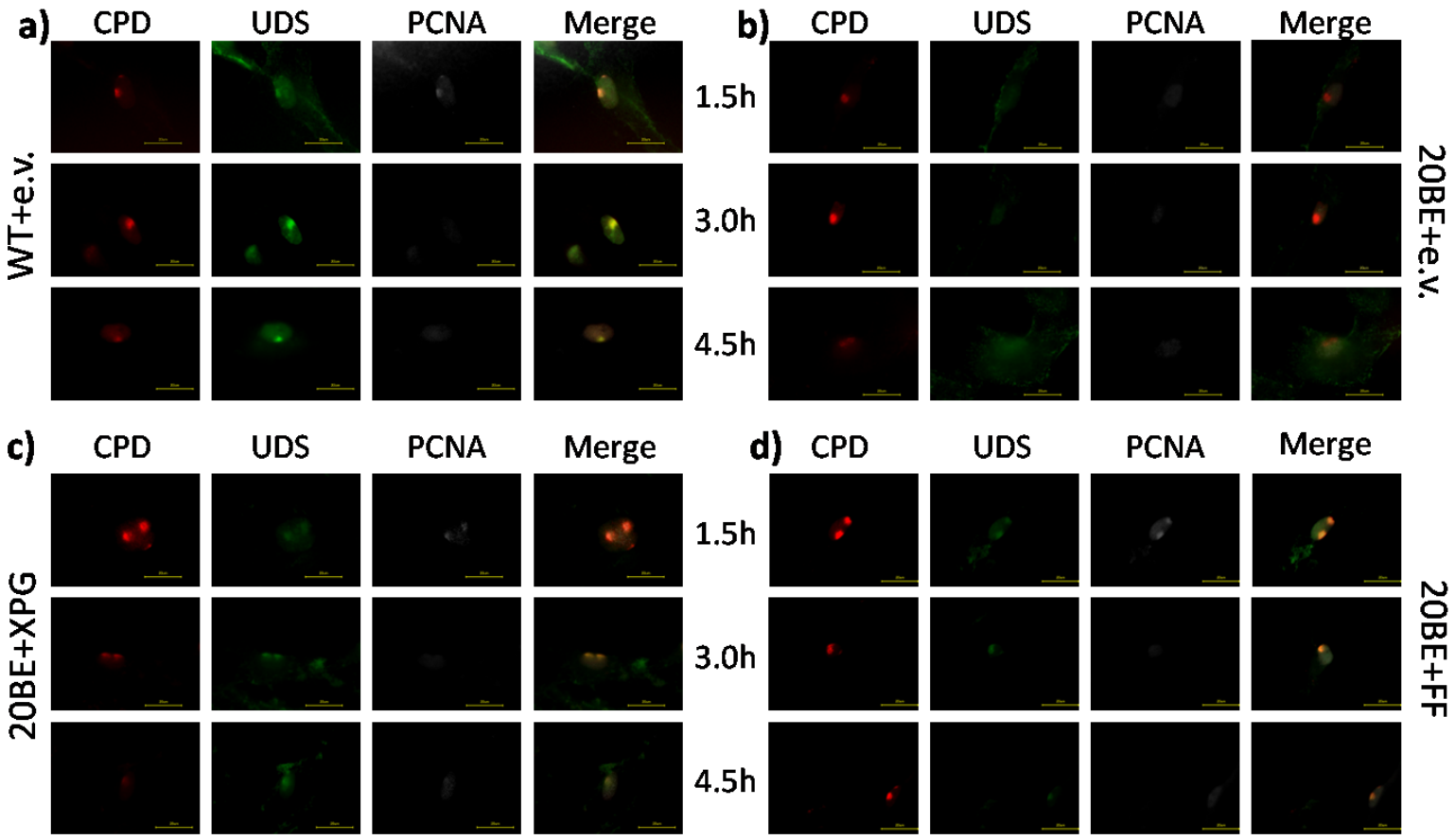

Figure 25: Triple staining (Triple-IF) of wt and XP20BE cells at different points in time after UV irradiation

A) WT fibroblasts, b) XP20BE + empty vector (e.v.), c) XP20BE + XPG, d) XP20BE + FF. Triple staining was performed like described in the methods section (see chapter 3.5.4). Red dots indicateUV derived DNA damages (CPDs), the green spots are UDS and the monograph picture displays PCNA. ( $h$ $=$ hours, yellow scale $=20 \mu \mathrm{m}$ )

In wildtype fibroblasts (see Figure 25a) as well as XPG complemented XP20BE cells (see Figure 25c) a clear merge between the DNA repair synthesis derived green UDS spots with the red cyclobutane pyrimidine dimers (CPD) (see chapter 1.2.3) spots, indicating DNA repair synthesis until this point in time after UVC irradiation (at all point in time 1.5 to $4.5 \mathrm{~h}$ ). Furthermore PCNA could be seen, to a 
certain extent, co-localized with the UV lesion and the UDS incorporation (at point in time 1.5 and 4.5h). Three hours after irradiation a clear CPD spot, marks the existence of DNA damage, and is observed as well as the UDS signal, demonstrating incorporated DNA nucleotides in this time period. No accumulated PCNA is observed at this point in time, independent of the PIP-box status of XPG, maybe indicating a periodical occurrence of repair (see Figure $25 c$, d).

The empty vector control (see Figure 25b) displays neither UDS nor PCNA accumulation in merge with a CPD spot in the observed period. This reveals a XPG dependency of the cells to perform the triple staining observed in Figure 25 a and c.

Surprisingly, the FF mutant (see Figure 25d) displayed a recruitment of PCNA as well as DNA repair synthesis activity like wt XPG. However, the difference between the quantitative UDS results (see Figure 21) and the qualitative triple IF assay is debated in the discussion (see chapter 5).

Taken together, these results indicate the independency of the C-terminally located canonical PIPbox for both, PCNA recruitment as well as DNA repair synthesis. However, an endonuclease defective XPG (E791A) or a truncated XPG isoform VI were neither able to recruit PCNA nor lead to DNA repair synthesis (data not shown).

\subsection{Investigations of other nucleases as a backup mechanism with regard to XPG Isoform VI}

In order to investigate the mechanism that enables the endonuclease defective and PCNA interaction compromised splice variant IsoVI of XPG to complement XP20BE cells (see chapter 4.2.3), I examined the effects of (co-)transfected candidate genes (see chapter 1.5, Table 14) on the UDS level two hours after irradiation.

Single transfection of $D N A 2$ or Fen1 (or its endonuclease defective mutant D181A) displays no significant difference in comparison to the empty vector (see Figure 26a) (Mann-Whitney-U-test). The IsoVI+e.v. (isoform and empty vector in a ratio of 1:2) positive control, as well as the transfection with wt-XPG and wt fibroblast cells transfected with the empty vector, show a highly significant increase of UDS levels compared to the empty vector ( $p<0.001$; Mann-Whitney-U-test). 

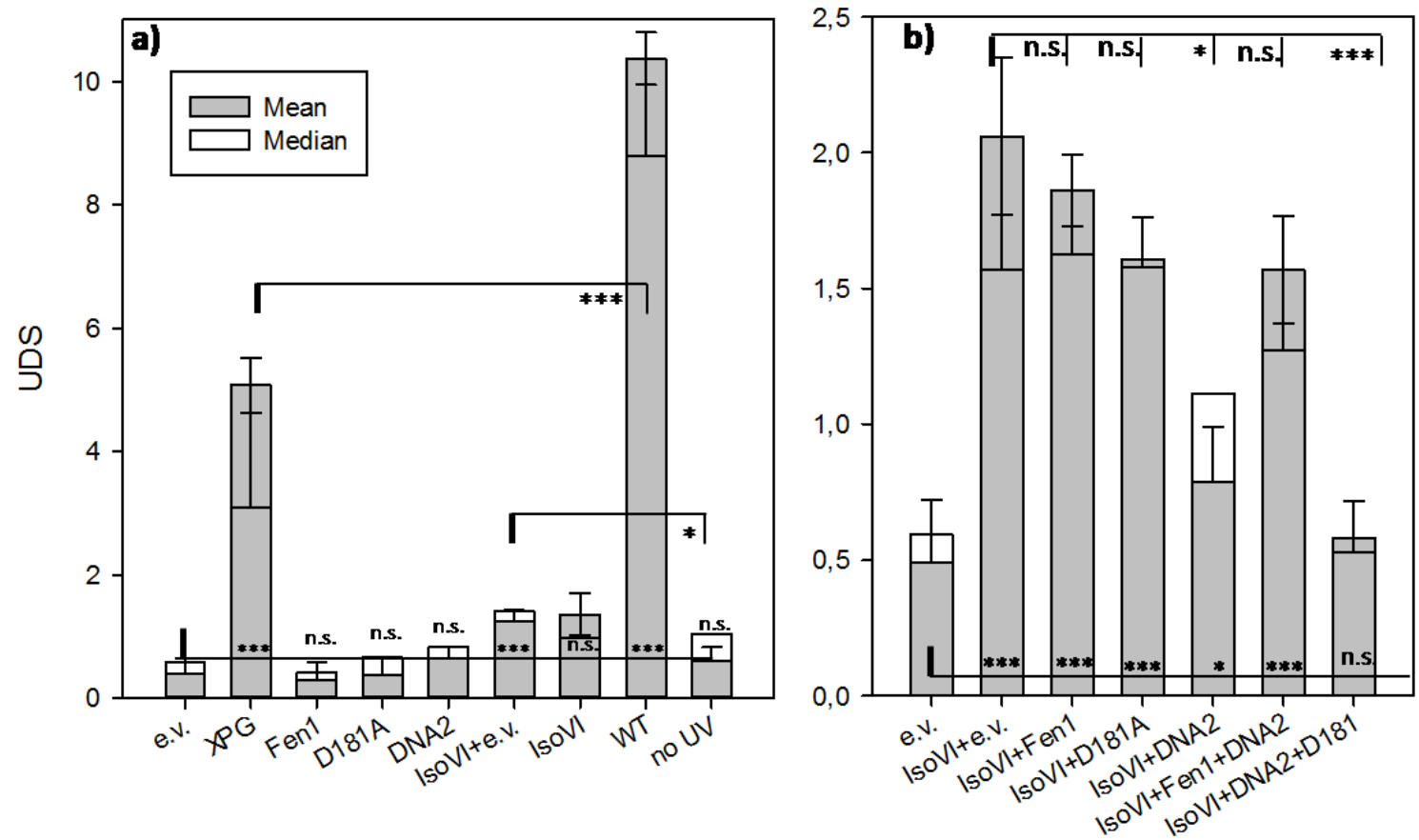

Figure 26: Assessment of UDS with respect to NER capability of Fen1 and DNA2

A) Illustrates controls, b) co-transfections of IsoVI plus Fen1 and/or DNA2. The gray bars represent the mean values of UDS of XP20BE nuclei (see chapter 3.5.2). The cells were transiently transfected with several XPG mutants (see chapter 4.2.1). The small black horizontal lines or white box of each bar indicate the median value two hours after irradiation. Significance levels were correlated using the Mann-Whitney U-Test for abnormally distributed series of measurements $\left({ }^{* * *}=p<0.001\right.$, $* *=p<0.01,{ }^{*}=p<0.05$, n.s. $=$ not significant; e.v. = empty vector). The small fat vertical lines, in the left of each significance comparison, mark the reference group.

Figure 26b illustrates the effect of co-transfection of two or three plasmids at once into XP20BE cells. The transfection of three plasmids at a single blow is routinely done and works well in the context of the HCR assay (see chapter 3.5.1). The double transfection of Isoform VI with Fen1 or its respective endonuclease defective mutant $D 181 \mathrm{~A}$ displays no significant difference compared to IsoVI alone and complements XP2OBE cells (Mann-Whitney-U-test). The co-transfection of IsoVI and DNA2 reduces the complementation in a significant manner but still enables IsoVI to complement the XPG defect (both $\mathrm{p}<0.05$; Mann-Whitney-U-test). The triple transfection of IsoVI, DNA2 and either Fen1 or D181A exhibits the clear result that the D181A mutant disturbs the DNA synthesis and thus complementation.

Taken together, these findings give a hint for the NER activity of Isoform VI in dependency of the endonuclease activity of Fen1. Thus, Fen1 is maybe suitable for a backup endonuclease to overcome a partial XPG defect. 


\section{Discussion}

The XPG protein is a key player in cell nuclei, comprising endonucleolytic as well as structural functions during DNA repair and transcription (see chapter 1.4). The aim of this study was to survey the behavior of XPG during NER by

- first, the exploration of the Xeroderma pigmentosum patient XP172MA in order to broaden and expand the spectrum of physiologically occurring XPG mutations as a model for XPG protein functions (see chapter 4.1),

- second, the genetic characterization of the PCNA-XPG interaction in terms of the XPG ability to catalyze DNA repair synthesis and facilitate NER (see chapter 1.3.2). This comprises the Nterminal PIP-UBM box as well as the C-terminal PIP-box (see chapter 4.2),

- third, the study of an alternative pathway during the very last steps of NER in dependency of the presence of a functionally constricted XPG and the involvement of Fen1 and DNA2 (see chapters 1.5, 4.3).

\subsection{General remarks}

XPG is essential for nuclear function. It interacts with a lot of diverse proteins and plays a role in DNA repair and basal transcription (see chapter 1.4.1). In line with its functional relevance, XPG patients are very scarce, even among the rare hereditary recessive disorder Xeroderma Pigmentosum itself. Patients often comprise the more severe XP/CS phenotype depending on the length of the residual $X P G$ allele.

Furthermore, very little is known about the appearance of XPG during NER, amongst other reasons, because of no available crystal structure. Especially the picture of the PCNA - XPG binding originates from studies regarding Fen1 family members (see chapter 1.5.1.1). Critical questions remain unanswered, e.g. how many monomers of (ubiquitinated) PCNA are bound by XPG or if there is a possibility for a handover between the two separated XPG PCNA-interaction domains (see chapter 4.2.1). However, XPG is designated to bind one monomer of PCNA in the depicted model and the ubiquitination of PCNA was assumed to be obligatory during (stalled) NER and is part of the regulation of interactions (see chapters 1.3.5, 1.3.6).

XP20BE primary fibroblasts, used as the XPG deficient host during this study, comprises only a residual 138AA (and 11AAs) XPG allele and displays a good cell behavior regarding proliferation and cell stature in the early passages (4-7), followed by a rapid decrease in cell viability in the late passages (8-12). Presumably, this decrease rests on the essential status of XPG for the cell 
metabolism, because XPG is a multiplayer with many interactions and functions, e.g. during transcription and DNA repair (see chapter 1.4). It is remarkable that the genotype of XP2OBE resulted in a phenotype compatible with life. However, the XP20BE patient died after 6 years displaying severe CS symptoms and no skin cancers (Moriwaki et al, 1996).

\section{General remarks concerning the functional assays used during this thesis}

\section{Host cell reactivation}

During Host cell reactivation (HCR) assays, the cells were transiently transfected with XPG (mutants) and reportergene expression plasmids (e.g. firefly luciferase) at the same time. Three days after transfection, the Transcription coupled repair (TCR) was measured as described (see chapter 3.5.1). The (potentially different) influence of the respective diverse XPG variants regarding transcription (see Figure 7) can be excluded. The arithmetical division of the damaged plasmid with an undamaged co-transfected second reporter gene plasmid, carrying the same promoter like the firefly plasmid, should be sufficient for normalization. The used dose during this approach is very high $\left(750 \mathrm{~J} / \mathrm{m}^{2}\right.$, applied on naked DNA) and requires NER to repair error free in sufficient amounts. However, XP2OBE cells transfected with the empty vector did not result in a detectable reportergene expression above background.

\section{Unscheduled DNA synthesis}

The Unscheduled DNA synthesis (UDS) of genomic DNA was measured after low dose UV irradiation $\left(20 \mathrm{~J} / \mathrm{m}^{2}\right.$ ) directly applied onto living cells (see chapter 3.5.2). All cells in growth phase were measured (see Figure 15), but just half of the cells showed XPG-dependent DNA repair synthesis. The reason lies in the transient transfection approach. This - like it is postulated by the manufacturer of the transfection reagent (Qiagen) - normally leads to a transfection efficacy of about $50-60 \%$, with a trend towards a decreasing rate using higher passages of the XP20BE cells (data not shown). The result was a population of abnormally distributed UDS value measurements.

To overcome that deficiency in comparison to stable transfected cell lines used by other groups, at least 300 nuclei derived from at least three independent transfections were estimated per XPG mutant and an appropriate statistical significance test was performed (Mann-Whitney-U-Test).

Additionally, every UDS nucleus measurement was subtracted with the respective cytosolic background fluorescence of the cell to yield a specific UV-dependent DNA repair synthesis rate. The influence of S-phase dependent DNA repair synthesis was excluded by omitting these cells as described (see chapter 3.5.2). The UDS rates reflect the presence of DNA synthesis until this point in 
time, with no information about termination of NER, the length of the synthesized DNA stretch, or NER accuracy.

\section{Comparison of UDS and HCR}

To compare these two assays in summary, the UDS displays short term genomic DNA repair activity (two hours) with no regard to the length (bp) or correctness of the synthesis, whereas HCR measures the long term repair capacity (three days) of correct NER in a transcriptional context (reportergene plasmid, requires correct protein coding). This leads to the conclusion, that if there is any UDS measured in dependency of a certain XPG mutant, which was not able to complement XPG deficient cells by HCR, DNA repair synthesis (UDS) promotes no XPG cleavage (partial synthesis) or has to be error prone (see Table 15, see Figure 8, see chapter 1.3.6).

Moreover, XPG acts at the very end of the NER pathway and plays a major role in both subpathways of NER, in GGR and TCR. However, due to its tight association with TFIIH it is probably recruited more easily if attached near to TFIIH in a transcriptional context. This would simplify complementation during HCR assays, not during UDS assays (see chapter 1.3.4).

\section{Triple-fluorescence staining}

The triple-fluorescence staining (Triple-IF) was established at the very end of the thesis with regard to the clarification of possible kinetic differences between PCNA recruitment and DNA repair synthesis (see chapter 3.5.4). The assay detects the local UV-derived DNA-damage (CPD), the PCNA sliding clamp and UDS at the same time in one cell. However, if any UDS is seen at any point in time, this accounts for the presence of PCNA prior to UDS with regard of its processive role during DNA synthesis (see chapter 1.3.5).

The assay was conducted qualitatively and has been performed only once prior to the completion of this thesis. With respect to the quantitative approach of HCR and UDS and the transientity of the transfection approach, the Triple-IF results should not be overrated. Additionally, in contrast to normal UDS $\left(20 \mathrm{~J} / \mathrm{m}^{2}\right)$, Triple-IF deals with a high dose of local DNA damage $\left(150 \mathrm{~J} / \mathrm{m}^{2}\right)$ in the chromosomal DNA. One reason for the strong UDS at different points in time observed can be due to the presence of Exo1 during NER (see chapter 1.5.1.2).

\subsection{XP172MA provides insights into XPG-TFIIH interaction}

A new disease-causing allele was identified in the very rare XP/CS complementation group $\mathrm{G}$, a novel and unusually large deletion mutation (genomic deletion of exons 2-5) leading to a truncated but in- 
frame XPG protein with stable expression and nuclear localization, but no functional repair activity (Lehmann, 2013; Schubert et al, 2013).

Fibroblast cells of respective patient XP172MA, exhibited a reduced post-UV survival as it was expected with regard to the phenotype and previously reported by UDS results ( $3 \%$ of wildtype, data not shown) (see Figure 17). The viability of XP172MA fibroblasts was strongly impaired even at low doses of UVC irradiation with a $L D_{50}$ value of $4 \mathrm{~J} / \mathrm{m}^{2}$ and $L D_{90}$ of $12 \mathrm{~J} / \mathrm{m}^{2}$. In comparison to this, the $L D_{50}$ of WT fibroblasts was estimated at $180 \mathrm{~J} / \mathrm{m}^{2}$ (data not shown) whereas cells from XPC patients exhibit a $L D_{50}$ value of about $30 \mathrm{~J} / \mathrm{m}^{2}$. The striking impact of the loss of XPG function is quite obvious and consistent with behavior of, also severely affected, other XPG/CS patient fibroblasts utilizing the MTT assay, reported by our group before (Schafer et al, 2013a; Schafer et al, 2013b). However, the postUV survival reflects the overall ability of the cells to cope with UV-derived DNA damages and involves several cellular mechanisms (NER, DDR, and apoptosis). The viability after UV irradiation of XP172MA fibroblast cells was very low and consistent with the severe XP/CS phenotype of the patient. In order to specifically estimate the NER capacity of these cells, Host cell reactivation was performed.

Patient XP172MA displayed a barely detectable NER value $(<0.1 \%)$ and was assigned to the XPG complementation group via HCR (see Figure 17). The introduction of wt-XPG into the cells restored NER capability to $50-60 \%$ compared to wildtype, consistent with the observed transfection efficacy during this transient approach and clearly demonstrating XPG deficiency. Additional experiments where the larger XP172MA allele (see Figure 18) was cloned out of the patient's mRNA (cDNA) of the patient into an expression vector and investigated regarding the complementation ability of the mutant protein in another XPG deficient cell line (XP20BE) under my supervision, revealed no complementation ability on empty vector level (Lehmann, 2013). This clearly suggests that the observed XP/CS phenotype, derived from the defective XPG protein, is independent of the genetic background.

To further characterize the XPG patient mutation which is disease-causing in XP172MA, sequencing on genome and mRNA (cDNA) level was performed. The sequencing results showed the occurrence of a minor and a major variant in the patient (see Figure 18). Both variants exhibit in frame exon skipping from exon1 to exon 6 due to a huge homozygous chromosomal deletion (6.7kbp). The longer variant, expressed to minor extent, showed a deletion of the respective amino acids 29-175. The shorter variant, expressed to the major extent, retained only 17 amino acids of exon 1 . It remains speculative, but the low expression-level variant is possibly due to splice variation, because of the homozygous nature of the deletion. 
As mentioned above, we decided to investigate the behavior of the larger variant during the master thesis of Janin Lehmann. It was shown in another study that a XPG splicevariant, expressed to a smaller extent, was able to rescue the phenotype to a certain extent (Thorel et al, 2004). Consistent with the severe XP/CS phenotype of the patient no NER activity of the cloned variant in crosscomplementing experiments was detected (HCR, no complementation (like empty vector),(Lehmann, 2013)).

There is a greatly established genotype-phenotype correlation of the severe XPG/CS phenotype with the deficiency of the XPG patient's gene products in TFIIH binding (see chapter 1.4.1). With regard to the reduced post-UV survival and the compromised NER capacity of the living cells, I propose that the disease-causing mutation of this patient leads to the inability to bind TFIIH (see chapter 1.1.2). The interaction of the N-terminus of XPG with TFIIH (XPB, XPD) has been earlier investigated during in vitro studies (lyer et al, 1996; Sarker et al, 2005).

In conclusion, the XPG-TFIIH interaction is so far designated to the amino acids 1-85 (see chapter 1.4.2). By genotype-phenotype correlation, this study greatly suggests to narrow down the functionally important XPG interaction domain to the amino-acids 30-85, which are designated to the endonucleolytic active $\mathrm{N}$-domain. Because of the strong correlational evidence, we have already published these data (British Journal of Dermatology, under revision) with no need for further interaction studies.

\subsection{Genetic characterization of the functional XPG-PCNA interaction during NER}

In the following chapter, I discuss the genetic characterization of the PCNA-XPG interaction in terms of XPG's ability to catalyze DNA repair synthesis and facilitate NER (see chapter 1.3.4.4). This comprises the N-terminal PIP-UBM box as well as the C-terminal PIP-box (see chapters 1.4.2, 4.2).

\subsubsection{The C-terminal PIP-box of XPG is required for quick DNA repair synthesis but is unimportant for complementation of XPG}

By comparison of the UDS and HCR results obtained during this study, I demonstrated that the Cterminal PIP-box is required for immediate UV response but not for the functionality of XPG during TCR or in response to local high-dose UV irradiation.

Both PCNA C-terminal interaction defective XPG mutants (Del, FF) used during this study were subcloned from plasmids provided by Prof. Ronal Gary (UNLV, USA) into the standardly used 
pcDNA3.1(+) vector (Gary et al, 1999; Gary et al, 1997). The results of the truncated Del mutant are discussed later (see chapter 5.4.2).

The FF clone is an artificially created XPG mutant optimized for defective PCNA binding in vitro. The same mutations of the respective Fen 1 aminoacids result in ineffective PCNA binding and show the same effect in the respective XPG mutant, due to the highly conserved character of these residues (personal correspondence with Ron Gary, UNLV, USA) (see Figure 12). Furthermore, this XPG peptide was able to compete with p21 (PCNA inhibitor) for PCNA binding, underlining the conserved nature of this interaction, like it was supposed from the PCNA-interacting Peptide (PIP) sequence alignment (see chapter 6.2).

\section{Results obtained during this thesis with FF mutants}

Utilizing UDS, compared to the empty vector, FF was able to restore UV damage response slightly but significant. This observation indicates the ability of this in vitro PCNA interaction defective XPG fulllength mutant to facilitate DNA repair synthesis in living cells, assuming the actual model of ongoing DNA repair synthesis during the intermediate state of NER (see Figure 8). However, compared to wtXPG or endonuclease deficient E791A, the FF mutant displayed a strongly reduced UDS rate (see Figure 23). The corresponding double mutant, who combines endonuclease defectiveness with PCNA binding deficiency from the results of in vitro studies, behaved like the FF single mutant (see chapter 4.2.1). These results indicate an impairment of immediate UV response (until two hours after irradiation) in dependency of the loss of PIP-box mediated PCNA binding, which was mutated in the FF clone.

The in vitro E791A endonuclease defective mutant of XPG permits a higher rate of UDS compared to FF at an earlier point in time (two hours, UDS) (see chapter 5.4.1). The corresponding double mutant $\mathrm{E} / \mathrm{FF}$ behaved like the FF mutant alone, underlining that the rate-limiting mutation for UDS is the one in the PIP-box motif. Furthermore, DM_E/FF behaved like E791A only during HCR assays, indicating that the respective E791A mutation is responsible the reduction of the repair capacity of this mutant.

In order to clarify the possible divergence between PCNA recruitment and the start of DNA repair synthesis during NER, I decided to investigate a simultaneous triple staining of CPDs (DNA damage), PCNA and UDS, in dependency of XPG mutants transfected into XP20BE cells (see Figure 25). The FF mutant displayed a recruitment of PCNA and simultaneous UDS activity like WT. An UDS-CPD merge picture was observed at all points in time. However, in WT and FF, PCNA was missing after three hours indicating either transfection problems due to the one-try challenge (see general) or a periodical repair action. 
I did not detect a discrepancy between temporal PCNA recruitment and DNA synthesis, because PCNA spots without UDS were not detected during triple staining. This result was unsurprising because of the processive nature of PCNA-dependent DNA synthesis (see chapter 1.3.5). Nevertheless, these results are not completely consistent with the quantitative analysis of the UDS rate two hours after irradiation (see Figure 23), which displays a significantly reduced rate compared to wildtype XPG.

One explanation for the observed divergence between the results obtained by UDS and Triple-IF, lies in the competition of XPG with p21 for PCNA binding (see chapters 1.3.3, 1.4, 6.2). The occurrence of local high-dose UV-derived DNA damage (Triple-IF) requires much more chromatin remodeling activity at one spot compared to low-dose cellular damage (UDS). The C-terminal Pip-box of XPG has been shown to compete with p21 for binding and is abolished in presence of the FF-mutation resulting in an accumulation of p21. This should lead to subsequent p300 hyper-acetylation and Histone acetyl transferase (HAT) activation. A second explanation for the increase in DNA repair synthesis, in dependency of the FF-mutant during the Triple-IF assay, resembles favoritsm of the Exo1 pathway performing end resection under these circumstances (see chapter 1.5.1.2). The involvement of the p21 pathway was not investigated during this thesis. Thus, it is highly speculative and ignored for the illustration of the model.

However, the Triple-IF results were approved by the complementation rate, measured three days after transfection of XP20BE cells with the UV-damaged reporter gene plasmid (see chapter 3.5.1). The FF repair value was comparable to wt-XPG. The repair rate was significantly enhanced compared to empty vector control as well as to endonuclease defective E791A.

\section{Results obtained by other research groups with the FF mutant}

In contrast to our study, Fagbemi and colleagues utilized immunofluorescence studies with the same FF mutation in XPG. They found out, that PCNA foci formation and 6-4PP removal of the FF mutant was comparable to WT, suggesting a rapid PCNA recruitment and depletion from local UV damage, utilizing immunofluorescence studies (Fagbemi, 2012). This is in line with our Triple-IF staining in dependency of the FF mutant. This accounts for a different behavior of this mutant in presence of high-dose local UV damage in comparison to low-dose UVC irradiation during UDS (see Figure 25).

The HCR results of my thesis are in consistency with previous findings by Gary et al using a comparable reporter gene based system. They also observed only a slight reduce in the Host cell reactivation capacity in XPG-deficient UV135 cells by PCNA-interaction compromised mutants of XPG (R992A/E) (Gary et al, 1997). The HCR findings presented here are also in line with the effect on 64PP removal in dependency of the XPG FF mutant in another dissertation (Fagbemi, 2012). In that 
study, the FF mutant behaved like wt XPG with a strong reduction of 6-4PP spots within the first six hours after irradiation. By all means, the presence of the FF mutation alone results not in an errorprone pathway, due to the complementation results using HCR (see model).

Taken together, especially comparing the UDS and HCR results obtained during this study, these findings demonstrate that the C-terminal PIP-box is required for immediate UV response but not for the functionality of XPG to perform accurate NER in transiently transfected primary fibroblasts.

\subsubsection{The functional N-terminal PCNA-XPG interaction is required for optimal NER}

The data presented here indicates importance of the functional, partly ubiquitin-derived, XPG-PCNA interaction, mediated by the conserved N-terminal PIP-UBM domain of XPG for functional NER. Furthermore, it implicates the existence of an (to a certain extent) obligatory occurrence of ubiquitinated PCNA during stalled NER. This observation leads to the proposal of a new XPG function in blocking the recruitment of translesion polymerases.

The C-terminally located highly conserved PIP-box is essential for complete DNA repair synthesis two hours after irradiation (UDS) but dispensable for complementation of XPG-deficiency during HCR assays (see Table 15). The FF mutant was shown to be PCNA binding defective in vitro. However, it was still able to facilitate DNA repair synthesis and NER during our experiments. The small N-terminal PIP-box (by length "a half" of the classical C-terminal PIP box) is located directly beside the also highly conserved ubiquitin binding domain (UBM) in the N-terminus of XPG. The influence of this PIP-UBM domain was estimated using HCR and UDS (see chapters 1.4.2, 3.5.1, 3.5.2, 4.2).

\section{Results obtained during this thesis with PIP-UBM mutants}

PIP mutation as well as UBM mutations (see chapter 4.2.1) were both sufficient to compromise the immediate repair synthesis (UDS) of the respective full-length wildtype or mutant XPGs (see Figure 22a-c). Furthermore, the single mutation of the N-terminal PIP-box (L179A) seems to be more efficient in decreasing the UDS rate compared to the UBM mutations (L196A \& P197A) alone (see Figure 22a, c). Noteworthy, the ubiquitin molecule is attached to PCNA in a flexible fashion, which increases its availability for binding partners (Hibbert \& Sixma, 2012; Hishiki et al, 2009). One explanation might be that the small N-terminal PIP-box of XPG plays a crucial role in the positioning of the ubiquitin molecule.

The simultaneous mutation of both domains (PIP-UBM), either using wildtype or endonuclease defective E791A XPG template, did not display any additive effect during UDS. Noteworthy, the PIPUBM double mutant showed no significant difference compared to the E791A-PIP-UBM triple mutant. This suggests a great importance of the N-terminal PIP-UBM box for the immediate UV 
derived DNA damage repair and underlines the irrelevance of XPG endonuclease function for the start of repair synthesis.

In case of the FF double mutants, whenever a FF mutant was mutated further at the N-terminal PCNA interaction domains (PIP, UBM), no mutant, neither the one with the addition of the PIP mutation nor the other one with mutations of the UBM domain, was sufficient to decrease the UDS rate of these double mutants to a level of zero (empty vector level) (see Figure 22b). However, the respective FF-PIP-UBM triple mutant somehow seems to compensate the strong defect of the respective double mutants and shows UDS at the level of the FF single mutation. This fact will be discussed with regard to the HCR results of these mutants, which are rather controversal.

The HCR assay reflects the ability to deal with huge amounts of UV derived DNA damage on a plasmid in an error-free fashion in dependency of the used XPG mutants (see Figure 24). Mutagenesis of single domains, either PIP or UBM, decreased the complementation ability of XPG in XP2OBE cells about 50\%, comparable to the level of the respective triple mutant as well as to the single E791A mutation in an endonuclease defective XPG clone (see Figure 24a, c).

The addition of either the single PIP mutation or the UBM domain mutations to endonuclease defective E791A (with the exception of E791A-PIP) or the FF mutant decreased the complementation ability compared to the E791A or FF mutations alone (see Figure 24b, c). The triple mutants E791APIP-UBM and FF-PIP-UBM displayed an additive decrease compared to the double mutants.

Mutations of the N-terminal PIP and UBM domains of the preselected XPG Isoform VI had no effect on the UDS rate (see Figure 22d). The DNA repair synthesis rate was comparable to the one FF catalyzed. This suggests a different mechanism of complementation by IsoVI compared to full-length wt-XPG, E791A and FF. One explanation for this is the truncated nature of Isoform VI with an altered incision complex formation during NER.

\section{Results obtained by other research groups with PIP-UBM mutants}

The UBM domain of XPG was mutated by another research group in the same way as I did it and it was demonstrated that the mutations strongly compromise ubiquitin binding of XPG (Fagbemi, 2012). However, the clear proof of XPG's binding to specifically ubiquitinated PCNA (at the Lysine 164 ) is still pending, but due to the adjacent location of the N-terminal PIP-box the binding of ubiquitinated PCNA is highly recommended (personal communication with Prof. Orlando Schärer, Stony Brook university).

My results are consistent with the observation of Fagbemi and colleagues, where no PCNA foci formation was observed during immunofluorescence studies in dependency of the PIP mutant and 
the PIP-UBM double mutant. Nevertheless, the single UBM mutant displayed a significant rate of PCNA foci formation, comparable to the level that is produced by E791A (Fagbemi, 2012). Assuming that PCNA foci formation and start of UDS are connected very tightly, and with no regard to the possible differences in these assays (see chapter 5.1), even the quantitative observation made by Fagbemi is reproduced with the UDS approach used during this study (see Figure 22a, c).

My UDS results obtained with the FF-PIP and FF-UBM double mutants are not in line with the observation from the Schärer group, that the FF-UBM double mutant facilitates PCNA foci formation on a level comparable to wt XPG at early measuring times and E791A at the later ones (Fagbemi, 2012). The differences between the assays are discussed elsewere and indicate a potential diverse back-up mechanism for XPG's endonuclease function (see chapters 5.1, 5.4.1).

The results of the presented HCR measurements (see Figure 24) of the PIP and UBM single mutants are consistent with the observation of the Schärer research group. They showed that PIP mutations and UBM mutations alone and the respective double mutant as well as E791A and a FF-UBM mutant all displayed comparable rates of 6-4PP removal around 50\% compared to the wt-XPG as well as the FF mutation alone (personal communication with Prof. Orlando Schärer, Stony Brook University) (Fagbemi, 2012).

\section{Conclusion with respect to the functional XPG-PCNA interaction}

During this thesis, I discovered great evidence for the functional importance of XPG-PCNA interaction during the Nucleotide excision repair pathway. The interactions of the NER dual incision complex are manifold, highly organized and orchestrated.

The number of impaired XPG functions, no matter if PCNA/ubiquitin interaction- or endonucleasedefective, seems to have more influence on the functional NER than the type of the aminoacid substitution itself (see Figure 22, Figure 24). Two mutations in the XPG cDNA were sufficient to decrease the UDS as well as the HCR rate with a trend towards an even more decreased value with three mutations. I suggest that the interaction between (ubiquitinated) PCNA and XPG is multilayered, all contributing in part to the overall function of XPG during NER. This observation is used in analogy to the translesion polymerase Rev1 during the illustration of the model (see chapter 1.3.6).

The almost negative influence of PIP and UBM single mutant variants of XPG during UDS and HCR (see Figure 22, Figure 24) further suggests that the N-terminal PIP and UBM domains of XPG work in concert in order to facilitate NER and thus can be seen as one PIP-UBM domain. When a XPG with a higher number of mutations is present (three, PIP-UBM plus E791A or FF), the occurrence of PIP and UBM mutations becomes additive regarding the decreasing NER. 
The only exception within my results is the FF-PIP-UBM clone which leads to great new insights into XPG'S NER function. The UDS rate of the FF-UBM-PIP mutant was above the respective double mutants and comparably high to the FF mutation alone. This excludes XPG as the factor responsible for PCNA recruitment, because PCNA is needed for appropriate DNA synthesis (see chapter 1.3.5). This is in line with the observation that the clamp loader RFC is sufficient for recycling PCNA at stalled replication intermediates during in vitro studies. Therefore, I suggest that the clamp loader RFC is also able to load PCNA onto the DNA structure during stalled NER incision intermediates (see Figure 27) (Masuda et al, 2007).

The complementation ability during HCR was not enhanced compared to the empty vector control and strongly reduced to the FF mutation alone. This mutant is highly compromised in PCNA binding in an ubiquitin-dependent way.

Ubiquitination of PCNA is a well established enhancer for the recruitment of translesion polymerases (e.g. Rev1) (see chapter 1.3.6). The binding of Rev1 to PCNA was shown to be regulated by the number of interactions in the mutasome, and Rev1 was still able to facilitate binding with a decreased number of interactions (seven to four).

It is very plausible to explain the FF-UBM-PIP results with a proposal for a new function of XPG in the following fashion: it blocks the association of translesion polymerases with stalled NER intermediates via multiple interactions with PCNA. In the context of NER, translesion polymerases would use the undamaged strand as a template and perform DNA synthesis with very low accuracy (error-prone). The involvement of the UBM binding domain of XPG in this observation leads to the second proposal of obligatory ubiquitinated PCNA during NER, which is in line with former suggestions (Masuda et al, 2010; Soria et al, 2006). That designates XPG as the factor that restricts the activation of translesion polymerases after UV irradiation to S-phase.

\subsection{Investigations on a backup mechanism for the XPG endonuclease function}

The third aim of my thesis was to investigate a theoretically possible alternative pathway during the very last steps of NER depending on the presence of a functionally constricted XPG. I investigated the effects of DNA2 and Fen1 regarding their endonucleolytic NER activity.

\subsubsection{Endonuclease defective XPG (E791A) is able to complement XP20BE deficient cells}

Results obtained from analyzes of the E791A mutant indicate the existence of a back-up mechanism, constantly present in human (skin) cells, which is somehow able to complement the endonucleolytic deficiency of XPG (especially HCR). Furthermore, the results of UDS support the actual "cut-patchcut-patch" model during dual incision (see Figure 8). 


\section{Results obtained during this thesis with the E791A mutants}

During this study, I investigated the activity of the endonuclease defective XPG mutants E791A and D77A in living cells. Utilizing Unscheduled DNA synthesis, the immediate post-UV response (two hours) of transiently transfected XP20BE fibroblast cells, with expression plasmids encoding these mutants, was measured by visualization of incorporated nucleoside analogs during genomic repair synthesis. Both mutants, as well as the respective double mutant, displayed a significantly reduced level of UDS, which is consistent with the current model drawn by Staresincic and colleagues in 2009 (Staresincic et al, 2009). During the intermediate state of dual incision, the repair synthesis machinery, composed of RFC, PCNA, and Polymerase $\delta$, is recruited after the first $5^{\prime}$ cut upstream of the lesion, performed by XPF/ERCC1, and starts synthesis along the undamaged strand prior to the downstream cut 3' by XPG (see chapter 1.3.4.4).

The UDS rate I observed within XPG complemented XP20BE cells is consistent with the estimated transfection efficacy of about $50-60 \%$ in comparison to the UDS rate of wildtype fibroblasts of a healthy donor (see Figure 26). Especially E791A displayed a decreased level of 50-60\% UDS activity compared to wt-XPG, perfectly matching with the results of the Schärer research group, utilizing the classical UDS (there, incorporation of radioactive $\left[{ }^{3} \mathrm{H}-1^{\prime}, 2^{\prime}\right]$-thymidine was measured) (see Figure 22). This fact demonstrates the functionality of the assay used in this study and it supports the proposed "cut-patch-cut-patch" model for partial repair synthesis prior to the second XPG cleavage (see Figure 8). However, the apparent small increase (not significant) of UDS observed with the double mutant, compared to the endonuclease defective single mutants, could be up to common fluctuations in the underlying transient transfection approach as well as to the ordinarily occurring slight variations observed within this kind of DNA repair assays (personal communication with Prof. Kenneth Kraemer, NIH).

The Host cell reactivation of XP2OBE cells by XPG mutants revealed truly unexpected results. Both endonuclease defective single mutants as well as the respective double mutant were able to complement the XPG deficiency (see Figure 23). Both XPG variants containing the corresponding E791A mutation, showed a significantly reduced HCR level compared to wildtype XPG as well as to D77A, indicating the more severe influence of the E791A amino acid substitution on both mutants. Again, the complementation rate of wt-XPG in comparison to fibroblasts of a healthy donor (WT, see Figure 23) perfectly matches the estimated transfection efficacy of XP20BE cells of about $50-60 \%$ (GFP plasmid based estimation of transfection efficacy analyzed using immunofluorescence techniques, data not shown). 


\section{Results obtained by other research groups with E791A XPG mutants}

Constantinou et al demonstrate that both substitutions from acidic residues to alanine (D77A, E791A) were not able to fully restore UV resistance in XPG-deficient cells $\left({ }^{3} \mathrm{H}\right.$-thymidine incorporation, viability assay), whereas substitutions to acidic aminoacids did, suggesting a strong impairment of XPG function in vitro by these mutations (Constantinou et al, 1999). Indeed, both substitutions were definitely shown to be endonuclease defective in vitro. However, the study by Constantinou and coworkers used stably transfected lymphoblastoid cells derived from the XPG patients XP3BR and XP125LO, both carrying residual full-length XPG alleles and retaining a splicevariant with intron retention between the XPG aa 917-962 and the in vitro endonuclease function compromising (not abolishing) A792V mutation, respectively (Scharer, 2008b). In comparison to the XP20BE cells used during my study (remaining alleles: 138 and 11 aa), the endogenous gene product of these cells used by Schärer et al is quite large. Hence, a rather dominant positive influence of the E791A and D77A mutants on the truncated endogenous patient protein was measured. Two other amino acid substitutions at the same position (D77E and E791D, acidic to acidic) were able to restore UV resistance of XP20BE cells in the same study, utilizing the same techniques (Constantinou et al, 1999). However, the influence of E791A and D77A on XP20BE post-UV survival was not published or analyzed there.

Consistent with my findings, another dissertation reports the partly complementation of XPCS1RO (XPG/CS) cells by stable transfection of E791A and by utilization of 6-4PP removal studies (about 3040\% retained activity) (Fagbemi, 2012). Within the first six hours after UV irradiation E791A removed 6-4PPs like WT. This period was followed by a barely detectable removal of DNA damage until 24 hours. Furthermore, the occurrence of PCNA foci at sites of local DNA damage in dependency of XPG (mutants) was also observed during that study.

\subsubsection{XPG Isoform VI supports complementation of XPG deficient cells and requires Fen1 endonuclease activity}

The physiologically occurring spontaneous mRNA splicevariant isoform $\mathrm{VI}$, a truncated XPG variant, was able to perform UDS as well as HCR activity at a low level and is compromised in the presence of an endonuclease defective Fen1, possibly indicating the involvement of a DNA2/Fen1- dependent backup mechanism for the endonuclease function of XPG.

\section{The activity of truncated XPG versions during NER}

Two truncated XPG variants were investigated with regard to the UV damage response during this thesis. Isoform VI, on the one hand, cloned out of the cDNA pool of fibroblasts of healthy individuals 
and preselected for NER activity. Del, on the other hand, was created artificially [Del: (Gary et al, 1997), IsoVI: (Lehmann, 2013)].

Due to the fact that truncation mutations of XPG cause the loss of TFIIH binding ability and XP/CS $(1.1 .2,1.4)$, a reduced NER capacity was expected. In consistency with this assumption, both mutants showed a decreased level of UDS two hours after irradiation (see Figure 22).

Noteworthy, both mutants are able to localize in the nucleus, as it was estimated by immunofluorescence studies using GFP fusion proteins of these mutants (Lehmann, 2013). In addition to the two major C-terminal NLS signals of XPG, an N-terminal nuclear localization signal (NLS-A) was detected two decades ago (see Figure 10) (Knauf et al, 1996). This indicates the sufficiency of the $\mathrm{N}$-terminal NLS-site ( $40 \%$ activity) for nuclear transport as well as for the presence of Isoform VI in nuclei in vivo.

In case of Isoform VI, the UDS rate was obviously enhanced, but did not reach a significant increase compared to the empty vector control. However, the dilution of IsoVI with empty vector (1:2) prior to transfection gained a significantly enhanced UDS rate compared to the IsoVI alone (see Figure 26). One reason for this observation might be a small malfunction of NER, created by the overexpression of a recombinant protein. The length of the IsoVI splicevariant is comparable to the allele of the XPCS1LV XPG/CS patient (659 aa) who suffers from a comparable phenotype like XP20BE (died at age of 6.5 years). IsoVI has, despite its truncated nature, the ability to configure the NER dual incision complex in a fashion that enables the first 5' cleavage by ERCC1/XPF. The observed UDS rate with IsoVI accounts at least for the partial DNA repair, like it was observed with E791A (see chapter 1.3.4.4). The binding affinity of a truncated XPG variant, like Isoform $\mathrm{VI}$, during NER dual incision complexes, is expected to be lower than a full-length XPG variant. Hence, it is likely displaced more quickly and potentially restricts the stalling of the NER complex to a certain extent. Compared to E791A, isoform VI probably decreases the number of successful NER events but thereby affords the synthesis of a longer DNA stretch.

The HCR results displayed a similar picture. The physiological splicevariant of XPG IsoVI increased the repair of the reporter gene plasmid to a small extent but in a highly significant manner $(p=0.002)$. In contrast to that, the transfection of the artificially created Del mutant transferred no NER activity to XP20BE cells, which is consistent with previous findings utilizing a comparable luciferase reporter gene assay (Gary et al, 1997). Neither PCNA recruitment nor UDS was observed within the first 4.5 hours after local UV irradiation in dependency of the Isoform VI using Triple-IF staining (data not shown). 
Isoform VI is one of two physiological isoforms that, under my supervision, were preselected among seven isoforms (Lehmann, 2013) with the highest complementation ability in living cells using HCR. Due to the truncated stature of IsoVI protein, it is unable to perform the endonucleolytic cleavage itself and needs a helper nuclease for the termination of NER.

The occurrence of physiological isoforms of XPG in skin cells and the NER activity underline the possibility of the existence of a nuclear backup mechanism for the XPG endonuclease function during NER. Moreover, endonuclease deficient Isoform VI itself is an evolutionary developed backup mechanism for the structural function of XPG during NER and uses a helper nuclease to perform the cleavage (see fig27). The helper nuclease in this case is the backup mechanism for the XPG endonuclease function.

\section{Investigations regarding a backup mechanism for XPG endonuclease function}

In accordance with the literature, DNA2 and Fen1 were chosen as candidates for further investigations. They are well known for the orchestrated action of flap primer removal during Okazaki fragment maturation (Burgers, 2009). An influence by Fen1 on post UV survival of higher eukaryotes has been proposed before (Matsumoto, 2001). Moreover, Fen1 and XPG are classified in the same endonuclease family, sharing a lot of homologies and comparable activities regarding the endonuclease and C-terminal PCNA binding domains and abilities (see chapter 1.5.1.1). Additionally, S. pombe Fen1 was shown to be able to cut down flaps with a length of 9-11 nts and a TT dimer 5'cap (Yoon et al, 1999). Under the assumption of the "cut-patch-cut-patch" model of the intermediate state of dual incision (see Figure 8), considering that a stalled NER reaction causes a similar structure as during Okazaki fragment maturation (see Figure 14), it is quite plausible to investigate the influence of Fen1 (in cooperation with DNA2) on the repair capacity of transfected XPG deficient cells. First, the unwinding of the long flap (24-32 nts, size of displaced oligonucleotide during NER) is performed by DNA2 followed by a cleavage adjacent ( $\left.5^{\prime}\right)$ to the UV lesion. Second, this leaves exactly the structure of a short flap with damage cap, like it was shown to be cleaved by Fen1 in vitro (Tapias et al, 2004) (see chapter 1.5.1.1).

As I have mentioned before concerning the E791A mutant, it evolved to be a difficult task to investigate the hypothesis of a backup mechanism for XPG endonuclease function. However, due to the fact that during HCR assays three plasmids are transfected in an obligatory fashion, I decided to conduct UDS assays with XPG Isoform VI and additionally transfected DNA2 and Fen1 (or a Fen1 endonuclease defective mutant, D181A) to measure the indirect effect of the inhibition of the NER reaction by the endonuclease defective Fen1. 
Figure 26a displays the ability of Isoform VI to gain DNA repair synthesis after UVC irradiation. Multiple plasmid transfections, with additionally overexpressed Fen1 or D181A, displayed no difference compared to IsoVI diluted with the empty vector. However, addition of recombinant DNA2 protein to the cells results in a decreased IsoVI-dependent complementation in presence of endonuclease defective Fen1 (D181A) (see Figure 26b). This is consistent with the observation that Fen1 alone has no effect, neither enhancing nor reducing, regarding the repair capability of E791A (personal communication with Prof. Orlando Schärer, Stony Brook University).

All three proteins (IsoVI, Fen1, and DNA2) have a comparable size and are supposed to act as monomers during NER reactions (see Table 14). The addition of DNA2 alone to IsoVI reduced the repair ability. One explanation could be the negative influence of overexpression and the disturbance of the molecular ratios between the three molecules, resulting in a decreased (short-term) UDS rate. But indeed, these findings support a strong dependency on Fen1 during this reaction.

The indirect proof towards the requirement for the endonuclease function of Fen1, thus meaning the complementation of IsoVI endonuclease deficiency, is produced with the great and highly significant reduction of IsoVI+DNA2+D181A in comparison to IsoVI diluted with the empty vector.

I suggest that my Isoform VI data displays an evolutionary evolved backup mechanism for XPG's function. However, further investigations are needed to clearly demonstrate a DNA2/Fen1 involvement (see chapter 6.2).

\section{Conclusion with respect to a possible backup mechanism for endonuclease function during NER}

The results of the UDS analysis of the endonuclease defective XPG clones, and especially E791A, support the "cut-patch-cut-patch" model of a structural role of XPG to trigger XPF/ERCC1 5' incision independently of XPG's 3' endonuclease function, like it has been demonstrated in vitro several times (Staresincic et al, 2009; Wakasugi et al, 1997). XPF/ERCC1 is recruited to sites of UV damage repair in dependency of the presence of XPG and is able to perform the $5^{\prime}$ cut, which leaves a structure able to recruit the DNA synthesis machinery (see Figure 8).

It was rather unexpected that a truncated version of XPG is able to complement XPG deficient cells. A closer look at the difference between Del and Isoform VI gives a hint for the reason of this IsoVI complementation ability. The artificially created Del mutant is about 250 amino acids longer than Isoform VI. This leads to the additional lack of a part of the RPA binding domain in IsoVI and of the Cterminal TFIIH interaction domain (four components) as well as the I-domain, which is known to be important for endonuclease activity (see Figure 10). Furthermore, IsoVI comprises 11 additional amino acids in its C-terminus, derived from intron retention due to the diverse splicing (Emmert et al, 
2001). Overexpression studies of Del mixed with DNA2 and Fen1 displayed no enhancement of the UDS rate in comparison to the empty vector control or Del alone, underlining the repair defectiveness of Del during NER (data not shown). Taken together, the most plausible explanation for the inability of Del to complement during NER is the evolutionarily evolved character of XPG isoform VI.

The theoretical involvement of Fen1 and/or DNA2 in the resolution of the proposed stalled intermediate structure in presence of E791A should be further investigated in order to clarify which nuclease(s) complement XPG endonuclease deficiency. The approach of knocking down Fen1 or DNA2 using shRNAs failed (data not shown). The knockdown was very efficient but destroyed proliferation of the cells because of the involvement of both proteins during Okazaki fragment maturation (see Figure 14). This was deleterious for the transient transfection approach during this study, which needs cell division for DNA delivery to nuclei. Additionally, simultaneous overexpression of E791A, Fen1 and DNA2 in addition to the reportergene plasmids Firefly and Renilla during HCR reactions (see chapter 3.5.1) failed (data not shown). Our observations point towards the ineffectiveness in bringing more than three plasmids to one cell at once.

The involvement of Exo1 end resection, as it was proposed during stalled NER intermediates in yeast and human cells (see 1.3, 1.5.1.2), should be excluded within UDS assays. Due to the fact that Exo1 is known to resect the end of DNA, beginning at the free $5^{\prime}$ flap over a huge stretch of DNA, creating big gaps of ssDNA within chromosomal regions, the observed UDS levels should be expected to be much higher. Additionally, the Exo1- dependent pathway can be seen as the unfavorable way of resolution due to the deleterious character of occurrence of great (RPA covered) ssDNA stretches. This includes the sensitivity of DNA regarding hydrolysis and the activation of cell cycle checkpoints (see chapter 1.3.1). However, the occurrence of the Exo1 pathway during HCR is naturally unknown.

I propose that both pathways just exist in parallel and that there is a chance for both ways of resolution in one or another context. 


\section{Summary and conclusions}

I was able to narrow down the functionally important XPG interaction domain between TFIIH and XPG to the XPG amino-acids $30-85$ by performing genotype-phenotype correlation studies of a novel XPG patient (XP172MA).

Furthermore, the model depicted in 6.1. illustrates a strong hint towards a complex multifactoral NER intermediate state. Within this study, I clarified that

- first, XPG is not responsible for the recruitment of PCNA,

- second, the XPG-ubiquitin interaction is much more important during NER than XPG's endonuclease function, and

- third, IsoVI represents a physiologically relevant backup mechanism for XPG's structural function that is (as least in part) depending on Fen1's nuclease activity, one cellular backup mechanism for the endonuclease function of XPG.

I propose that the XPG-ubiquitin interaction blocks the association of translesion polymerases with NER intermediates and restricts UV-activated TLS polymerase recruitment to S-phase. The model illustrated in Figure 27 is a very plausible explanation that confers consistency to the results presented during this thesis. 


\subsection{Model for the function of XPG during stalled NER intermediates}

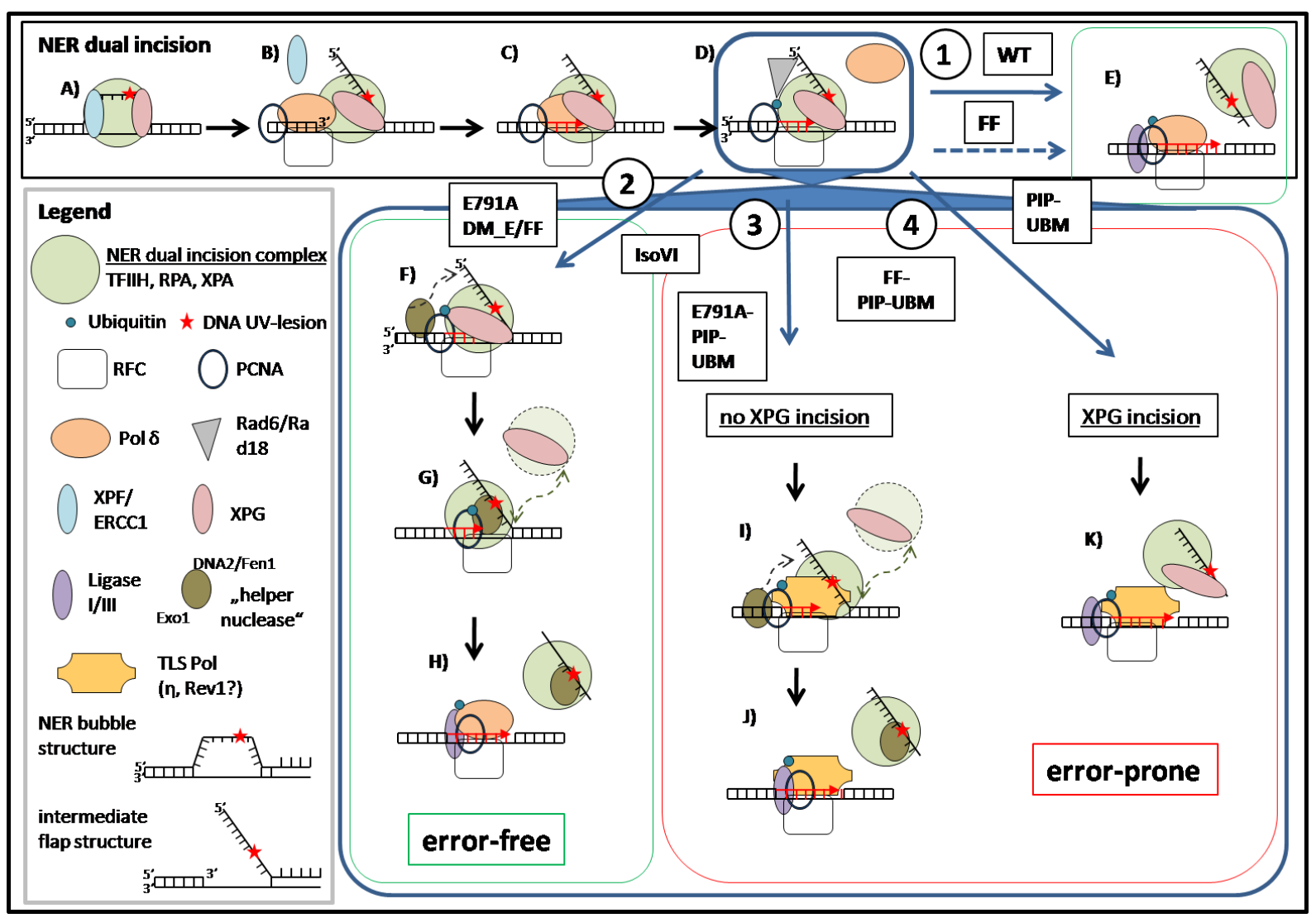

Figure 27: Model for the function of XPG during stalled NER intermediates

\subsubsection{General remarks concerning the model}

The model displays four different pathways of resolution in an error-free (green boxed) or errorprone (red boxed) fashion. Involved mutants in the pathways are named beside the arrows and the illustration between two boxes (green, red) indicates an involvement in both pathways.

Due to simplification of the involved proteins, three groups were created (see Legend of Figure 27):

- NER dual incision complex, "NER Holo complex" [TFIIH (10 subunits), RPA, XPA],

- "helper nuclease" [either DNA2/Fen1 or Exo1] (see chapter 1.5), and

- translesion polymerase [TLS polymerases $\kappa, \eta, \zeta$, เ or Rev1].

PCNA is seen like a molecular revolver barrel during this model, due to its circular nature and the presence as a trimer. Each monomer can be modified or carry a protein ("bullet"), and a simple turnaround of PCNA would subsequently deliver another reaction partner to (stalled) reaction complexes, no matter of in terms of replication or NER. 
The translesion polymerase involved in this case is currently unknown but potentially involves the TLS polymerases $k, \eta, \zeta$, เ or Rev1. The latter is a translesion polymerase, beside its unique function of cytosine incorporation activity, it is able to recruit other translesion polymerases (see chapter 1.3.6). These polymerases partly share sequence homologies with XPG and are known to bind also DNA and (ubiquitinated) PCNA in a XPG-like fashion. Especially Rev1 was shown to have four interactions in the replication complex, with PCNA (2), ubiquitin (1), and DNA (1), just like it is assumed for XPG during this work (see Figure 9). Due to the similarities in binding, I conclude that every interaction with ubiquitin, PCNA and DNA plays a role in the ability of XPG to perform successful NER. In the context of this NER dual incision model, translesion polymerases would use the undamaged strand as a template and perform DNA synthesis with very low accuracy (error-prone).

The N-terminal PCNA interaction (PIP) is important for NER. It potentially ensures the correct positioning of the whole machinery, in concert with the ubiquitin binding UBM box, prior to XPG's cleavage. The C-terminal PIP-box (FF-mutant) is responsible for the fast track of the assembled DNA repair synthesis machinery, possibly preventing ubiquitination of PCNA. The third interaction is the one between XPG's nuclease domains and DNA, which might also be important for positioning and a tight grip of XPG to the whole NER complex. Only if the complex is assembled in a sufficient position, this will lead to the (fast or slow) cut by XPG.

\subsubsection{Elucidation of the model}

The model of dual incision during NER, which is presented here (see Figure 27), is in great consistency with data presented in this study (see chapters 4.2, 4.3, especially Table 15). Furthermore, the current status of knowledge in the field of DNA repair and replication was taken into account. The result depicted in Figure 27 represents a model derived by genetic screening and is designated to be proofed in further biochemical experiments, which is discussed in the last chapter (see chapter 6.2).

The upper box of Figure 27, which is outlined black, depicts the status of NER during normal dual incision. A legend for the naming of the factors, depicted in the black and blue box, is illustrated at the lower end of the figure in a grey box. General assumptions are found in the respective section (see chapter 6.1.1).

A) The NER dual incision complex is completely recruited as well as both endonucleases XPF/ERCC1 and XPG (see chapter 1.3.4.4). The DNA displays a bubble structure and the complex is arranged in a correct fashion to perform the first ssDNA cut of the damaged strand 5 ' of the lesion. XPF-ERCC1 performs the first cleavage depending on the presence of a XPG, which is able to fulfill its structural function in order to sufficiently stabilize the NER incision complex. 
B) After the cleavage by XPF/ERCC1, the endonuclease is displaced by the DNA repair synthesis machinery consisting of PCNA, RFC and Pol $\delta$. The simple occurrence of a dsDNA-ssDNA junction with free $3^{\prime} \mathrm{OH}$ is sufficient for the recruitment of the clamp loader RFC, subsequent recruitment of the processivity factor PCNA and the polymerase (Masuda et al, 2007). RFC transfers the processivity to this machinery, especially to PCNA, whereas the polymerase itself displays a rather distributive character.

C) The actual model proposes the start of DNA repair synthesis prior to the performance of the second cut by XPG $3^{\prime}$ of the lesion (see chapter 1.3.4.4). The clash of the polymerase $\delta$ and XPG (no direct interaction published) should be sufficient to displace the polymerase as well as RPA from the undamaged strand. This might be the trigger for the cleavage of XPG. However, the disruption of processivity of the polymerase in this situation leaves a stalled NER reaction, which involves the same core factors like a replication event (PCNA, RFC, and Pol $\delta)$.

D) A stalled complex in the first instance creates time for molecular reactions to occur (blue outline). The presence of RPA, whether bound to the ssDNA of the bubble structure or after displacement at the ssDNA flap, is sufficient to recruit the Rad6/Rad18 ubiquitin ligase, which is known to ubiquitinate PCNA monomers at the conserved K164 residue (see chapters 1.3.5, 1.3.6). The excised oligonucleotides after NER reactions were found to be coated with RPA, which leads to the conclusion that RPA is present in NER reactions until the second 3' (XPG) cut has been performed. Hence, $\mathrm{RPA}_{74}$ will be able to recruit Rad6/Rad18 (Sale et al, 2012). A possibility is that the same displaced RPA from the undamaged strand subsequently binds the damaged ssDNA flap.

Furthermore, my results in this thesis reveal a strong influence of the N-terminally located ubiquitin-mediated PCNA interaction domain of XPG regarding the DNA repair activity measured by two different assays in living cells. Mutations in these domains decreased the NER capacity of XPG, at least to an extent of an essentially compromised endonuclease deficient XPG.

I propose a model that involves obligatory ubiquitination of PCNA during NER, at least due to the high potential of leaving enough time for secondary modifications. Not every NER event necessarily involves ubiquitination of PCNA but the number of these events is in a physiologically relevant area and an ubiquitin molecule is most probably bound by the $\mathrm{N}$ terminally located PIP-UBM box of XPG. This leads to faster or slower NER reactions, but in turn results in the cleavage by XPG. In presence of the FF XPG mutant (C-terminal PIP box) the stalling is prolonged, compared to the WT XPG, and the transition to E) is slower, indicated by the blue dotted arrow. This can increase the probability of PCNA ubiquitination. 
The blue outlined, stalled dual incision reaction, was chosen as an origin to reconsider the different XPG cases regarding the reactions at a NER bubble. The big blue box in Figure 27 illustrates the proposal of an error-prone (red box) as well as two error-free (green box) pathways of resolution of a stalled NER reaction in dependency of diverse XPG mutants (or an isoform).

\subsubsection{Error-free pathways of resolution}

Intermediate resolution pathway one (wt-XPG, FF, PIP-UBM):

E) After the $3^{\prime}$ endonucleolytic cleavage performed by XPG, the NER dual incision complex as well as XPG are displaced together with the excised damage containing oligonucleotide. The highly conserved canonical PIP-box in the C-terminus (which was mutated using the FF mutant during this thesis) promotes an ubiquitination-free immediate repair synthesis in an error-free fashion. A XPG variant with mutations in the PIP-UBM box is still capable of performing the fast track resolution of the stalled intermediates by using the C-terminal PIPbox. Polymerase $\delta$ is recruited again as well as a ligase for nick sealing. The latter by another monomer of PCNA. Error-free repair synthesis is completed.

\section{Intermediate resolution pathway two (E791A, IsoVI, DM_E/FF):}

F) The presence of an endonuclease defective full-length XPG (E791A) is able to assemble the NER incision complex correctly. Due to its complete (ubiquitin-)PCNA binding ability (three interactions), it should be able to protect the reaction from the recruitment of translesion polymerases during the stalling of the reaction, which is supposed to be longer due to the requirement for the recruitment of the helper endonuclease, most probably by PCNA's free third monomer. However, the suggested pathway of resolution is error-free. The addition of the FF-mutation to E791A has an increasing effect on the complementation rate (HCR) compared to E791A alone, whereas the UDS rate is decreased, with the same level of DNA repair synthesis like the FF. This underlines the depicted model in Figure 27. The addition of the FF mutation apparently enables E791A the performance of an error-free pathway more easily by making it easier for the helper nuclease to be recruited.

In presence of the XPG splicevariant IsoVI, a physiologically relevant truncated version of $X P G$, the NER reaction complex is assembled correctly but in a decreased frequency and less stable compared to full-length XPG. However, the NER complex is formed if IsoVI is present. It is very likely that the stalling of the complex is sufficient enough for ubiquitination of PCNA and/or direct recruitment of the helper endonuclease by the DNA structure and a subsequent displacement of IsoVI as well as the NER Holo complex. 
G) The recruitment of a helper nuclease (Fen1/DNA2 or Exo1) should in turn trigger the release of the endonuclease deficient XPG (E791A) because the DNA structure has to become available somehow. Probably the release of XPG is accompanied by the recruitment of the DNA polymerase $\delta$ and the displacement of the NER dual incision complex.

The XPG isoform VI also enables this error-free pathway of resolution, due to its ubiquitin binding ability. This pathway is accompanied by the rapid displacement of IsoVI, the NER Holo complex as well as the helper endonuclease, and the dual incision product. However, the further mutagenesis of IsoVI in the PIP-UBM domain did not result in a decrease rate of UDS or HCR repair, indicating the unimportance of this interaction domain regarding the binding of ubiquitinated PCNA. This is potentially due to the lack of TFIIH interaction of the Cterminus as well as to the loss of RPA binding activity and DNA binding with the I-Domain (see chapter 1.4.2). One explanation might be that IsoVI presence somehow favors the direct recruitment of helper nucleases. In comparison, the artificially truncated mutant Del is not able to complement at all (see chapter 4.3 ). This raises the possibility of an evolutionarily developed backup mechanism for the XPG function during NER by Isoform VI. However, the reduced binding ability and the low complementation rate (HCR) of IsoVI also account for the error-prone resolution pathway three (see chapter 3.5.1). Within this apparent discrepancy lies the potential of an evolutionarily developed mechanism to fine-tune the rate of mutations in the genome.

H) The cleavage is performed. The oligonucleotide containing the lesion is displaced as well as the involved factors. One monomer of PCNA is free and probably able to recruit ligase I/III, setting everything up for the nick sealing after the termination of DNA repair synthesis.

\subsubsection{Error-prone pathways of resolution}

\section{Intermediate resolution pathway three (IsoVI, FF-PIP-UBM, E791A-PIP-UBM):}

In the presence of PCNA binding deficient XPG in living cells (FF-PIP-UBM) the incision complex is stably built due to sufficient interactions with the NER Holo complex. Moreover, the possibility of a triggered XPG incision would be plausible if the clash with polymerase $\delta$ would lead to the subsequent XPG cleavage.

I) If the clash between Pol $\delta$ and XPG is not sufficient for the activation of the XPG $3^{\prime}$ endonuclease activity and further PCNA interaction is needed, the FF-UBM-PIP is hardly compromised to perform the cleavage. In that case XPG is displaced, together with or without the NER Holo complex, and a helper nuclease is recruited via their interaction with PCNA, probably bound to another PCNA monomer. The recruitment of the translesion 
polymerase should be sufficient to displace XPG and also probably the NER dual incision complex.

The helper endonuclease is recruited to the DNA structure and cuts specifically (DNA2/Fen1) or resects the end of a damaged strand over long stretch (Exo1). Beside its observed role during local high-dose UV repair, in contrast to Fen1, I did not find any hint in the literature for the ability of Exo1 to digest NER intermediate structures in vitro. However, in terms of avoidance of deleterious long stretches of (RPA coated) SSDNA in presence of DNA damage (possible problems: hydrolysis, recombination, activation of cellular checkpoints), the genome integrity would possibly favor a Fen1/DNA2 based mechanism.

J) The error-prone action of TLS polymerases on the undamaged template, which incorporates a wrong nucleotide every 30 bases (see chapter 1.3.6), is sufficient to create mutations. However, the lesion-containing oligonucleotide is displaced as well as the NER Holo complex and the helper nuclease.

The proposed involvement of IsoVI in this error-prone pathway displays an evolutionarily developed backup mechanism for XPG which is able to fine-tune the mutation frequency of cells in order to gain new functional changes in terms of genetic adaption.

The addition of the E791A mutation to a PIP-UBM mutant is supposed to act in this pathway. The lack of ubiquitin binding and the inability to cleave DNA results in a highly error-prone DNA synthesis, displayed by comparison of the UDS and HCR results, with a moderate post-UV DNA synthesis and a very low reporter gene expression, respectively.

\section{Intermediate resolution pathway four (FF-PIP-UBM, PIP-UBM):}

K) If XPG cleavage is performed in presence of ubiquitin binding defective mutants (PIP-UBMs), after the clash with the polymerase, the subsequent displacement of the NER dual incision complex, XPG, and of the excised oligo enables the recruitment of error-prone translesion polymerases, stimulated by the unbound ubiquitinated PCNA. I conclude, because of the data presented during this study, that XPG blocks the recruitment of TLS polymerases via its interaction with ubiquitinated PCNA. In case of the artificially created FF-UBM-PIP triple mutant, XPG's mechanism of action provides a small but significant amount of error-prone TLS synthesis (see Table 15). However, the strikingly decreasing effect by mutations of the $\mathrm{N}$ terminal PIP domain alone leaves the possibility that the proposed direct interaction between PCNA and the N-terminally located PIP of XPG is highly important for the positioning of the complex in order to bind ubiquitin, because the ubiquitin molecule was found to be attached to PCNA in a flexible fashion. The PIP-UBM double mutant is still able to 
perform the error-free pathway using the C-terminal PIP domain during the quick proceeding of the stalling (see chapter 6.1.2.1). This mutant complements during NER with about 50\% repair activity compared to wildtype (HCR, UDS) which reflects probably a 50-50 chance for both pathways.

\subsection{Future perspectives}

The first clear evidence that has to be produced is the direct interaction of XPG with ubiquitinated PCNA. This can involve Co-IP studies using protein cell extracts of XPG-variant transfected cells or direct in vitro interaction studies with purified recombinant proteins. There are in vitro methods available to even control the ubiquitination status of each monomer. This can solve the question about the number of bound PCNA monomers of XPG. Furthermore, such studies would provide more evidence regarding the question which mechanism triggers cleavage by XPG during dual incision (Figure 8).

Additionally, the involvement of several translesion polymerases in dependency of the XPG mutants has to be studied. Especially Rev1 is a great candidate. This can be conducted by recruitment kinetics using immunofluorescence techniques or by in vitro NER reactions. The latter would be similarly well suited to clarify if the FF-PIP-UBM XPG mutant is able to perform an incision during NER. The establishment of stable transfected cell lines would be a big advantage for immunofluorescence studies. However, the immortalization of the XP20BE cells, the basis for these types of experiments, is currently under preparation.

The ongoing debate about the existence of a second backup mechanism involving DNA2/Fen1 could also be solved in vitro. The control of the composition of the reaction factors is able to reproduce a mechanism involving either Exo1 or DNA2 and Fen1. It is quite essential to clarify the occurrence or non-occurrence of Exo1 at NER reaction bubbles, e.g. in G1-phase (measured in UDS). The ongoing translesion synthesis as well as the processive end resection by Exo1 both would be able to increase the UDS rate. However, the effect of Exo1 would be much bigger compared to normal or translesion synthesis and is probably excluded by the avoidance of grain nuclei measurements for UDS ratios. To set up an in vitro experiment which is able to track NER synthesis from single events should also be able to solve this problem.

Additional analysis of a quadruple mutant (E791A-FF-PIP-UBM) with the same techniques as well as analysis of several other mutants in the triple IF stainings could be useful for the Exo1 debate on the one hand and on the other hand for confirmation or disproval of the model presented here (see Figure 27). 
XPG splicevariants are an ongoing field of research in our lab. The establishment of specific qRT-PCRs for each isoform and the measurement of respective expression levels in different individuals and tissues are temporarily under investigation. Splicevariants are a highly suitable protein species for the investigation as cancer biomarkers, as has been reported before (Brinkman, 2004; Zhang et al, 2013b). Further research in this field is highly recommended and can lead to a new prognostic value of XPG regarding chemotherapeutic response (Hu et al, 2014). Furthermore, the possible influence of the isoform VI as a backup mechanism for several other XPG functions (see chapter 1.4) can be tested.

I was not able to detect PCNA without UDS during Triple-IF studies. However, the assay theoretically enables also the detection of other proteins than PCNA and can be a new strong tool for the clarification of molecular interplay at NER intermediates.

In general, the transient transfection approach of primary fibroblasts is very useful for the relatively fast screening of many mutants regarding the NER activity in living cells. However, for statistically better quantitative results with UDS and recruitment kinetics, additional work with stable transfected cell lines is highly recommended. Nevertheless, the screening for genetic functions, like it was executed during this thesis, is much more feasible with the used "high-throughput" transient transfection approach.

Beside other factors, XPG is also involved in chromatin remodeling in the context of UV damage repair (see chapter 1.3.3). The PCNA-XPG-p21-p300 signal transduction is potentially an additional layer of regulation of NER activity. XPG is acetylated in vitro in a p300-dependent fashion. This attachment is inhibited by PCNA and is in turn dependent on the interaction between p21 and PCNA (Tillhon et al, 2012). XPG is further known to inhibit p21 binding to PCNA (see chapters 1.3.5, 1.4), most probably via the conserved PIP-box in the C-terminus of XPG, mutated in the FF mutant (see chapter 1.4.2) (Gary et al, 1997). P21 was also shown to transfer Histone acetyl transferase (HAT) activity to $\mathrm{p} 300$ which is required for the chromatin remodeling in response to UV irradiation.

Secondary modifications were excluded from the model, as well as the functional p21 involvement (see chapter 1.5.1.1). Like Fen1 (see chapter 1.5.1.1), XPG comprises a lot of theoretical phosphorylation sites and, so far, nothing is known about their functionalities (http://www.phosphosite.org). Due to its close relationship to Fen1, especially the phosphorylation of XPG is most probably functionally relevant and nothing about it is published, making it extremely time-consuming to investigate. 
I already successfully applied for a post-doc start-up grant from the DFG. Furthermore, a large research application for the investigation of splicevariants of NER protein at Deutsche Krebshilfe e.V. has already been submitted and involves the plan to conduct biochemical in vitro assays as mentioned above. 


\section{Bibliography}

Aboussekhra A, Biggerstaff M, Shivji MK, Vilpo JA, Moncollin V, Podust VN, Protic M, Hubscher U, Egly JM, Wood RD (1995) Mammalian DNA nucleotide excision repair reconstituted with purified protein components. Cell 80: 859-868

Actis M, Inoue A, Evison B, Perry S, Punchihewa C, Fujii N (2013) Small molecule inhibitors of PCNA/PIP-box interaction suppress translesion DNA synthesis. Bioorg Med Chem 21: 1972-1977

Ahmad J, Cooke MS, Hussieni A, Evans MD, Patel K, Burd RM, Bleiker TO, Hutchinson PE, Lunec J (1999) Urinary thymine dimers and 8-oxo-2'-deoxyguanosine in psoriasis. FEBS Lett 460: 549-553

Arab HH, Wani G, Ray A, Shah ZI, Zhu Q, Wani AA (2010) Dissociation of CAK from core TFIIH reveals a functional link between XP-G/CS and the TFIIH disassembly state. PLoS One 5: e11007

Arase S, Kozuka T, Tanaka K, Ikenaga M, Takebe H (1979) A sixth complementation group in xeroderma pigmentosum. Mutation research 59: 143-146

Araujo SJ, Nigg EA, Wood RD (2001) Strong functional interactions of TFIIH with XPC and XPG in human DNA nucleotide excision repair, without a preassembled repairosome. Mol Cell Biol 21: 22812291

Araujo SJ, Tirode F, Coin F, Pospiech H, Syvaoja JE, Stucki M, Hubscher U, Egly JM, Wood RD (2000) Nucleotide excision repair of DNA with recombinant human proteins: definition of the minimal set of factors, active forms of TFIIH, and modulation by CAK. Genes Dev 14: 349-359

Ariza RR, Keyse SM, Moggs JG, Wood RD (1996) Reversible protein phosphorylation modulates nucleotide excision repair of damaged DNA by human cell extracts. Nucleic acids research 24: 433440

Bae SH, Bae KH, Kim JA, Seo YS (2001) RPA governs endonuclease switching during processing of Okazaki fragments in eukaryotes. Nature 412: 456-461

Balajee AS, Machwe A, May A, Gray MD, Oshima J, Martin GM, Nehlin JO, Brosh R, Orren DK, Bohr VA (1999) The Werner syndrome protein is involved in RNA polymerase II transcription. Mol Biol Cell 10: 2655-2668

Balakrishnan L, Bambara RA (2013) Flap endonuclease 1. Annu Rev Biochem 82: 119-138

Balakrishnan L, Stewart J, Polaczek P, Campbell JL, Bambara RA (2010) Acetylation of Dna2 endonuclease/helicase and flap endonuclease 1 by p300 promotes DNA stability by creating long flap intermediates. J Biol Chem 285: 4398-4404 
Bergink S, Toussaint W, Luijsterburg MS, Dinant C, Alekseev S, Hoeijmakers JH, Dantuma NP, Houtsmuller AB, Vermeulen W (2012) Recognition of DNA damage by XPC coincides with disruption of the XPC-RAD23 complex. J Cell Biol 196: 681-688

Bertani G (1951) Studies on lysogenesis. I. The mode of phage liberation by lysogenic Escherichia coli. Journal of bacteriology 62: 293-300

Bessho T (1999) Nucleotide excision repair 3' endonuclease XPG stimulates the activity of base excision repairenzyme thymine glycol DNA glycosylase. Nucleic acids research 27: 979-983

Bienko M, Green CM, Crosetto N, Rudolf F, Zapart G, Coull B, Kannouche P, Wider G, Peter M, Lehmann AR, Hofmann K, Dikic I (2005) Ubiquitin-binding domains in Y-family polymerases regulate translesion synthesis. Science 310: 1821-1824

Biertumpfel C, Zhao Y, Kondo Y, Ramon-Maiques S, Gregory M, Lee JY, Masutani C, Lehmann AR, Hanaoka F, Yang W (2010) Structure and mechanism of human DNA polymerase eta. Nature 465: 1044-1048

Biggerstaff M, Wood RD (1999) Assay for nucleotide excision repair protein activity using fractionated cell extracts and UV-damaged plasmid DNA. Methods Mol Biol 113: 357-372

Birnboim HC, Doly J (1979) A rapid alkaline extraction procedure for screening recombinant plasmid DNA. Nucleic acids research 7: 1513-1523

Bohr VA, Smith CA, Okumoto DS, Hanawalt PC (1985) DNA repair in an active gene: removal of pyrimidine dimers from the DHFR gene of $\mathrm{CHO}$ cells is much more efficient than in the genome overall. Cell 40: 359-369

Bradford MM (1976) A rapid and sensitive method for the quantitation of microgram quantities of protein utilizing the principle of protein-dye binding. Anal Biochem 72: 248-254

Bradford PT, Goldstein AM, Tamura D, Khan SG, Ueda T, Boyle J, Oh KS, Imoto K, Inui H, Moriwaki S, Emmert S, Pike KM, Raziuddin A, Plona TM, DiGiovanna JJ, Tucker MA, Kraemer KH (2011) Cancer and neurologic degeneration in xeroderma pigmentosum: long term follow-up characterises the role of DNA repair. J Med Genet 48: 168-176

Branzei D, Foiani M (2010) Maintaining genome stability at the replication fork. Nat Rev Mol Cell Biol 11: 208-219

Brash DE, Rudolph JA, Simon JA, Lin A, McKenna GJ, Baden HP, Halperin AJ, Ponten J (1991) A role for sunlight in skin cancer: UV-induced p53 mutations in squamous cell carcinoma. Proceedings of the National Academy of Sciences of the United States of America 88: 10124-10128 
Bravo R, Celis JE (1980) A search for differential polypeptide synthesis throughout the cell cycle of HeLa cells. J Cell Biol 84: 795-802

Brinkman BM (2004) Splice variants as cancer biomarkers. Clin Biochem 37: 584-594

Britt AB (1996) DNA Damage and Repair in Plants. Annu Rev Plant Physiol Plant Mol Biol 47: 75-100

Brown AL, Lee CH, Schwarz JK, Mitiku N, Piwnica-Worms H, Chung JH (1999) A human Cds1-related kinase that functions downstream of ATM protein in the cellular response to DNA damage. Proceedings of the National Academy of Sciences of the United States of America 96: 3745-3750

Budd ME, Campbell JL (2000) The pattern of sensitivity of yeast dna2 mutants to DNA damaging agents suggests a role in DSB and postreplication repair pathways. Mutation research 459: 173-186

Budzowska M, Kanaar R (2009) Mechanisms of dealing with DNA damage-induced replication problems. Cell Biochem Biophys 53: 17-31

Burgers PM (2009) Polymerase dynamics at the eukaryotic DNA replication fork. J Biol Chem 284: 4041-4045

Campbell C, Quinn AG, Ro YS, Angus B, Rees JL (1993) p53 mutations are common and early events that precede tumor invasion in squamous cell neoplasia of the skin. J Invest Dermatol 100: 746-748

Cann IK, Ishino Y (1999) Archaeal DNA replication: identifying the pieces to solve a puzzle. Genetics 152: $1249-1267$

Chapados BR, Hosfield DJ, Han S, Qiu J, Yelent B, Shen B, Tainer JA (2004) Structural basis for FEN-1 substrate specificity and PCNA-mediated activation in DNA replication and repair. Cell 116: 39-50

Chatterjee S, Stochaj U (1998) Diffusion of proteins across the nuclear envelope of HeLa cells. Biotechniques 24: 668-674

Chehrehasa F, Meedeniya AC, Dwyer P, Abrahamsen G, Mackay-Sim A (2009) EdU, a new thymidine analogue for labelling proliferating cells in the nervous system. J Neurosci Methods 177: 122-130

Chen J, Larochelle S, Li X, Suter B (2003) Xpd/Ercc2 regulates CAK activity and mitotic progression. Nature 424: 228-232

Chen X, Paudyal SC, Chin RI, You Z (2013) PCNA promotes processive DNA end resection by Exo1. Nucleic acids research 41: 9325-9338 
Christmann M, Tomicic MT, Origer J, Kaina B (2005) Fen1 is induced p53 dependently and involved in the recovery from UV-light-induced replication inhibition. Oncogene 24: 8304-8313

Cleaver JE (1968) Defective repair replication of DNA in xeroderma pigmentosum. Nature 218: 652656

Cleaver JE (2000) Common pathways for ultraviolet skin carcinogenesis in the repair and replication defective groups of xeroderma pigmentosum. J Dermatol Sci 23: 1-11

Cloud KG, Shen B, Strniste GF, Park MS (1995) XPG protein has a structure-specific endonuclease activity. Mutation research 347: 55-60

Cohen SM, Ellwein LB (1991) Genetic errors, cell proliferation, and carcinogenesis. Cancer Res 51: 6493-6505

Coin F, Auriol J, Tapias A, Clivio P, Vermeulen W, Egly JM (2004) Phosphorylation of XPB helicase regulates TFIIH nucleotide excision repair activity. EMBO J 23: 4835-4846

Coin F, Marinoni JC, Egly JM (1998a) Mutations in XPD helicase prevent its interaction and regulation by $\mathrm{p} 44$, another subunit of TFIIH, resulting in Xeroderma pigmentosum (XP) and trichothiodystrophy (TTD) phenotypes. Pathol Biol (Paris) 46: 679-680

Coin F, Marinoni JC, Rodolfo C, Fribourg S, Pedrini AM, Egly JM (1998b) Mutations in the XPD helicase gene result in XP and TTD phenotypes, preventing interaction between XPD and the p44 subunit of TFIIH. Nat Genet 20: 184-188

Coin F, Oksenych V, Egly JM (2007) Distinct roles for the XPB/p52 and XPD/p44 subcomplexes of TFIIH in damaged DNA opening during nucleotide excision repair. Mol Cell 26: 245-256

Coin F, Oksenych V, Mocquet V, Groh S, Blattner C, Egly JM (2008) Nucleotide excision repair driven by the dissociation of CAK from TFIIH. Mol Cell 31: 9-20

Compe E, Egly JM (2012) TFIIH: when transcription met DNA repair. Nat Rev Mol Cell Biol 13: 343-354

Consortium IHGS (2004) Finishing the euchromatic sequence of the human genome. Nature 431: 931-945

Constantinou A, Gunz D, Evans E, Lalle P, Bates PA, Wood RD, Clarkson SG (1999) Conserved residues of human XPG protein important for nuclease activity and function in nucleotide excision repair. $J$ Biol Chem 274: 5637-5648

Contreras JG, Madariaga ST (2003) Structure and the energy of base pairing in non-natural bases of nucleic acids: the azaguanine-cytosine and azaadenine-thymine base pairs. Bioorg Chem 31: 367-377 
Cooke MS, Evans MD, Burd RM, Patel K, Barnard A, Lunec J, Hutchinson PE (2001) Induction and excretion of ultraviolet-induced 8-oxo-2'-deoxyguanosine and thymine dimers in vivo: implications for PUVA. J Invest Dermatol 116: 281-285

Cooper PK, Nouspikel T, Clarkson SG, Leadon SA (1997) Defective transcription-coupled repair of oxidative base damage in Cockayne syndrome patients from XP group G. Science 275: 990-993

Cortez D (2005) Unwind and slow down: checkpoint activation by helicase and polymerase uncoupling. Genes Dev 19: 1007-1012

Craggs TD, Hutton RD, Brenlla A, White MF, Penedo JC (2013) Single-molecule characterization of Fen1 and Fen1/PCNA complexes acting on flap substrates. Nucleic acids research

D'Amours D, Jackson SP (2002) The Mre11 complex: at the crossroads of dna repair and checkpoint signalling. Nat Rev Mol Cell Biol 3: 317-327

D'Errico M, Parlanti E, Teson M, de Jesus BM, Degan P, Calcagnile A, Jaruga P, Bjoras $M$, Crescenzi $M$, Pedrini AM, Egly JM, Zambruno G, Stefanini M, Dizdaroglu M, Dogliotti E (2006) New functions of XPC in the protection of human skin cells from oxidative damage. EMBO J 25: 4305-4315

de Barros AC, Takeda AA, Chang CW, Kobe B, Fontes MR (2012) Structural basis of nuclear import of flap endonuclease 1 (FEN1). Acta Crystallogr D Biol Crystallogr 68: 743-750

de Laat WL, Appeldoorn E, Sugasawa K, Weterings E, Jaspers NG, Hoeijmakers JH (1998) DNA-binding polarity of human replication protein A positions nucleases in nucleotide excision repair. Genes Dev 12: 2598-2609

Dianov G, Bischoff C, Sunesen M, Bohr VA (1999) Repair of 8-oxoguanine in DNA is deficient in Cockayne syndrome group B cells. Nucleic acids research 27: 1365-1368

DiGiovanna JJ, Kraemer KH (2012) Shining a light on xeroderma pigmentosum. J Invest Dermatol 132: 785-796

Dionne I, Nookala RK, Jackson SP, Doherty AJ, Bell SD (2003) A heterotrimeric PCNA in the hyperthermophilic archaeon Sulfolobus solfataricus. Mol Cell 11: 275-282

DiRuggiero J, Santangelo N, Nackerdien Z, Ravel J, Robb FT (1997) Repair of extensive ionizingradiation DNA damage at 95 degrees $C$ in the hyperthermophilic archaeon Pyrococcus furiosus. Journal of bacteriology 179: 4643-4645

Dollfus H, Porto F, Caussade P, Speeg-Schatz C, Sahel J, Grosshans E, Flament J, Sarasin A (2003) Ocular manifestations in the inherited DNA repair disorders. Surv Ophthalmol 48: 107-122 
Drapkin R, Reardon JT, Ansari A, Huang JC, Zawel L, Ahn K, Sancar A, Reinberg D (1994) Dual role of TFIIH in DNA excision repair and in transcription by RNA polymerase II. Nature 368: 769-772

Dubaele S, Proietti De Santis L, Bienstock RJ, Keriel A, Stefanini M, Van Houten B, Egly JM (2003) Basal transcription defect discriminates between xeroderma pigmentosum and trichothiodystrophy in XPD patients. Mol Cell 11: 1635-1646

Dumaz N, Drougard C, Sarasin A, Daya-Grosjean L (1993) Specific UV-induced mutation spectrum in the p53 gene of skin tumors from DNA-repair-deficient xeroderma pigmentosum patients. Proceedings of the National Academy of Sciences of the United States of America 90: 10529-10533

Dunand-Sauthier I, Hohl M, Thorel F, Jaquier-Gubler P, Clarkson SG, Scharer OD (2005) The spacer region of XPG mediates recruitment to nucleotide excision repair complexes and determines substrate specificity. J Biol Chem 280: 7030-7037

Durando M, Tateishi S, Vaziri C (2013) A non-catalytic role of DNA polymerase eta in recruiting Rad18 and promoting PCNA monoubiquitination at stalled replication forks. Nucleic acids research 41: 30793093

Egly JM, Coin F (2011) A history of TFIIH: two decades of molecular biology on a pivotal transcription/repair factor. DNA Repair (Amst) 10: 714-721

Ehrhart JC, Gosselet FP, Culerrier RM, Sarasin A (2003) UVB-induced mutations in human key gatekeeper genes governing signalling pathways and consequences for skin tumourigenesis. Photochem Photobiol Sci 2: 825-834

Eller MS, Maeda T, Magnoni C, Atwal D, Gilchrest BA (1997) Enhancement of DNA repair in human skin cells by thymidine dinucleotides: evidence for a p53-mediated mammalian SOS response. Proceedings of the National Academy of Sciences of the United States of America 94: 12627-12632

Emmert S, Kraemer KH (2013) Do not underestimate nucleotide excision repair: it predicts not only melanoma risk but also survival outcome. J Invest Dermatol 133: 1713-1717

Emmert S, Leibeling D, Runger TM (2006) Syndromes with genetic instability: model diseases for (skin) cancerogenesis. J Dtsch Dermatol Ges 4: 721-731

Emmert S, Schneider TD, Khan SG, Kraemer KH (2001) The human XPG gene: gene architecture, alternative splicing and single nucleotide polymorphisms. Nucleic acids research 29: 1443-1452

Emmert S, Slor H, Busch DB, Batko S, Albert RB, Coleman D, Khan SG, Abu-Libdeh B, DiGiovanna JJ, Cunningham BB, Lee MM, Crollick J, Inui H, Ueda T, Hedayati M, Grossman L, Shahlavi T, Cleaver JE, Kraemer KH (2002) Relationship of neurologic degeneration to genotype in three xeroderma pigmentosum group G patients. J Invest Dermatol 118: 972-982 
Enzlin JH, Scharer OD (2002) The active site of the DNA repair endonuclease XPF-ERCC1 forms a highly conserved nuclease motif. EMBO J 21: 2045-2053

Essers J, Theil AF, Baldeyron C, van Cappellen WA, Houtsmuller AB, Kanaar R, Vermeulen W (2005) Nuclear dynamics of PCNA in DNA replication and repair. Mol Cell Biol 25: 9350-9359

Evans E, Fellows J, Coffer A, Wood RD (1997) Open complex formation around a lesion during nucleotide excision repair provides a structure for cleavage by human XPG protein. EMBO J 16: 625638

Eyboulet F, Cibot C, Eychenne T, Neil H, Alibert O, Werner M, Soutourina J (2013) Mediator links transcription and DNA repair by facilitating Rad2/XPG recruitment. Genes Dev 27: 2549-2562

Fagbemi A (2012) The Study of XPG Domains that Regulate The Late Steps of Nucleotide Excision Repair. Stony Brook University, Stony Brook University, Stony Brook University

Fagbemi AF, Orelli B, Scharer OD (2011) Regulation of endonuclease activity in human nucleotide excision repair. DNA Repair (Amst) 10: 722-729

Faili A, Aoufouchi S, Weller S, Vuillier F, Stary A, Sarasin A, Reynaud CA, Weill JC (2004) DNA polymerase eta is involved in hypermutation occurring during immunoglobulin class switch recombination. J Exp Med 199: 265-270

Fan J, Otterlei M, Wong HK, Tomkinson AE, Wilson DM, 3rd (2004) XRCC1 co-localizes and physically interacts with PCNA. Nucleic acids research 32: 2193-2201

Fan W, Luo J (2010) SIRT1 regulates UV-induced DNA repair through deacetylating XPA. Mol Cell 39: 247-258

Fearon ER, Vogelstein B (1990) A genetic model for colorectal tumorigenesis. Cell 61: 759-767

Finger LD, Blanchard MS, Theimer CA, Sengerova B, Singh P, Chavez V, Liu F, Grasby JA, Shen B (2009) The 3'-flap pocket of human flap endonuclease 1 is critical for substrate binding and catalysis. $J$ Biol Chem 284: 22184-22194

Fitch ME, Cross IV, Turner SJ, Adimoolam S, Lin CX, Williams KG, Ford JM (2003a) The DDB2 nucleotide excision repair gene product p48 enhances global genomic repair in p53 deficient human fibroblasts. DNA Repair (Amst) 2: 819-826

Fitch ME, Nakajima S, Yasui A, Ford JM (2003b) In vivo recruitment of XPC to UV-induced cyclobutane pyrimidine dimers by the DDB2 gene product. J Biol Chem 278: 46906-46910 
Fousteri M, Mullenders LH (2008) Transcription-coupled nucleotide excision repair in mammalian cells: molecular mechanisms and biological effects. Cell Res 18: 73-84

Fousteri M, Vermeulen W, van Zeeland AA, Mullenders LH (2006) Cockayne syndrome A and B proteins differentially regulate recruitment of chromatin remodeling and repair factors to stalled RNA polymerase II in vivo. Mol Cell 23: 471-482

Franklin A, Milburn PJ, Blanden RV, Steele EJ (2004) Human DNA polymerase-eta, an A-T mutator in somatic hypermutation of rearranged immunoglobulin genes, is a reverse transcriptase. Immunol Cell Biol 82: 219-225

Fregoso M, Laine JP, Aguilar-Fuentes J, Mocquet V, Reynaud E, Coin F, Egly JM, Zurita M (2007) DNA repair and transcriptional deficiencies caused by mutations in the Drosophila p52 subunit of TFIIH generate developmental defects and chromosome fragility. Mol Cell Biol 27: 3640-3650

Freudenthal BD, Gakhar L, Ramaswamy S, Washington MT (2010) Structure of monoubiquitinated PCNA and implications for translesion synthesis and DNA polymerase exchange. Nat Struct Mol Biol 17: $479-484$

Frosina G, Fortini P, Rossi O, Carrozzino F, Raspaglio G, Cox LS, Lane DP, Abbondandolo A, Dogliotti E (1996) Two pathways for base excision repair in mammalian cells. J Biol Chem 271: 9573-9578

Gaillard PH, Martini EM, Kaufman PD, Stillman B, Moustacchi E, Almouzni G (1996) Chromatin assembly coupled to DNA repair: a new role for chromatin assembly factor I. Cell 86: 887-896

Gaillard PH, Moggs JG, Roche DM, Quivy JP, Becker PB, Wood RD, Almouzni G (1997) Initiation and bidirectional propagation of chromatin assembly from a target site for nucleotide excision repair. EMBO J 16: 6281-6289

Gali H, Juhasz S, Morocz M, Hajdu I, Fatyol K, Szukacsov V, Burkovics P, Haracska L (2012) Role of SUMO modification of human PCNA at stalled replication fork. Nucleic acids research 40: 6049-6059

Galloway AM, Liuzzi M, Paterson MC (1994) Metabolic processing of cyclobutyl pyrimidine dimers and (6-4) photoproducts in UV-treated human cells. Evidence for distinct excision-repair pathways. J Biol Chem 269: 974-980

Gan GN, Wittschieben JP, Wittschieben BO, Wood RD (2008) DNA polymerase zeta (pol zeta) in higher eukaryotes. Cell Res 18: 174-183

Gary R, Kim K, Cornelius HL, Park MS, Matsumoto Y (1999) Proliferating cell nuclear antigen facilitates excision in long-patch base excision repair. J Biol Chem 274: 4354-4363 
Gary R, Ludwig DL, Cornelius HL, MacInnes MA, Park MS (1997) The DNA repair endonuclease XPG binds to proliferating cell nuclear antigen (PCNA) and shares sequence elements with the PCNAbinding regions of FEN-1 and cyclin-dependent kinase inhibitor p21. J Biol Chem 272: 24522-24529

Giannattasio M, Follonier C, Tourriere H, Puddu F, Lazzaro F, Pasero P, Lopes M, Plevani P, MuziFalconi M (2010) Exo1 competes with repair synthesis, converts NER intermediates to long ssDNA gaps, and promotes checkpoint activation. Mol Cell 40: 50-62

Giglia-Mari G, Zotter A, Vermeulen W (2011) DNA damage response. Cold Spring Harb Perspect Biol 3: a000745

Gillet LC, Scharer OD (2006) Molecular mechanisms of mammalian global genome nucleotide excision repair. Chem Rev 106: 253-276

Godon C, Mourgues S, Nonnekens J, Mourcet A, Coin F, Vermeulen W, Mari PO, Giglia-Mari G (2012) Generation of DNA single-strand displacement by compromised nucleotide excision repair. EMBO J 31: 3550-3563

Goosen N (2010) Scanning the DNA for damage by the nucleotide excision repair machinery. DNA Repair (Amst) 9: 593-596

Grasby JA, Finger LD, Tsutakawa SE, Atack JM, Tainer JA (2012) Unpairing and gating: sequenceindependent substrate recognition by FEN superfamily nucleases. Trends Biochem Sci 37: 74-84

Gratchev A, Strein P, Utikal J, Sergij G (2003) Molecular genetics of Xeroderma pigmentosum variant. Exp Dermatol 12: 529-536

Green MR, Sambrook J, Sambrook J (2012) Molecular cloning : a laboratory manual, 4th edn. Cold Spring Harbor, N.Y.: Cold Spring Harbor Laboratory Press.

Greenblatt MS, Beaudet JG, Gump JR, Godin KS, Trombley L, Koh J, Bond JP (2003) Detailed computational study of p53 and p16: using evolutionary sequence analysis and disease-associated mutations to predict the functional consequences of allelic variants. Oncogene 22: 1150-1163

Groisman R, Polanowska J, Kuraoka I, Sawada J, Saijo M, Drapkin R, Kisselev AF, Tanaka K, Nakatani Y (2003) The ubiquitin ligase activity in the DDB2 and CSA complexes is differentially regulated by the COP9 signalosome in response to DNA damage. Cell 113: 357-367

Guerrero-Santoro J, Kapetanaki MG, Hsieh CL, Gorbachinsky I, Levine AS, Rapic-Otrin V (2008) The cullin 4B-based UV-damaged DNA-binding protein ligase binds to UV-damaged chromatin and ubiquitinates histone H2A. Cancer Res 68: 5014-5022 
Guo Z, Kanjanapangka J, Liu N, Liu S, Liu C, Wu Z, Wang Y, Loh T, Kowolik C, Jamsen J, Zhou M, Truong K, Chen Y, Zheng L, Shen B (2012) Sequential posttranslational modifications program FEN1 degradation during cell-cycle progression. Mol Cell 47: 444-456

Guo Z, Qian L, Liu R, Dai H, Zhou M, Zheng L, Shen B (2008) Nucleolar localization and dynamic roles of flap endonuclease 1 in ribosomal DNA replication and damage repair. Mol Cell Biol 28: 4310-4319

Guo Z, Zheng L, Xu H, Dai H, Zhou M, Pascua MR, Chen QM, Shen B (2010) Methylation of FEN1 suppresses nearby phosphorylation and facilitates PCNA binding. Nat Chem Biol 6: 766-773

Haaf T, Schmid M (2000) Experimental condensation inhibition in constitutive and facultative heterochromatin of mammalian chromosomes. Cytogenet Cell Genet 91: 113-123

Halley DJ, Keijzer W, Jaspers NG, Niermeijer MF, Kleijer WJ, Boue J, Boue A, Bootsma D (1979) Prenatal diagnosis of xeroderma pigmentosum (group C) using assays of unscheduled DNA synthesis and postreplication repair. Clin Genet 16: 137-146

Hanahan D (1983) Studies on transformation of Escherichia coli with plasmids. Journal of molecular biology 166: 557-580

Hanahan D, Weinberg RA (2000) The hallmarks of cancer. Cell 100: 57-70

Hanawalt PC (1989) Preferential repair of damage in actively transcribed DNA sequences in vivo. Genome 31: 605-611

Hanawalt PC, Haynes RH (1967) The repair of DNA. Sci Am 216: 36-43

Hanawalt PC, Spivak G (2008) Transcription-coupled DNA repair: two decades of progress and surprises. Nat Rev Mol Cell Biol 9: 958-970

Hara R, Sancar A (2003) Effect of damage type on stimulation of human excision nuclease by SWI/SNF chromatin remodeling factor. Mol Cell Biol 23: 4121-4125

Harada YN, Shiomi N, Koike M, Ikawa M, Okabe M, Hirota S, Kitamura Y, Kitagawa M, Matsunaga T, Nikaido O, Shiomi T (1999) Postnatal growth failure, short life span, and early onset of cellular senescence and subsequent immortalization in mice lacking the xeroderma pigmentosum group $\mathrm{G}$ gene. Mol Cell Biol 19: 2366-2372

Hargreaves DC, Crabtree GR (2011) ATP-dependent chromatin remodeling: genetics, genomics and mechanisms. Cell Res 21: 396-420 
Harrigan JA, Wilson DM, 3rd, Prasad R, Opresko PL, Beck G, May A, Wilson SH, Bohr VA (2006) The Werner syndrome protein operates in base excision repair and cooperates with DNA polymerase beta. Nucleic acids research 34: 745-754

Harrington JJ, Lieber MR (1994) Functional domains within FEN-1 and RAD2 define a family of structure-specific endonucleases: implications for nucleotide excision repair. Genes Dev 8: 1344-1355

Hasan S, Hassa PO, Imhof R, Hottiger MO (2001a) Transcription coactivator p300 binds PCNA and may have a role in DNA repair synthesis. Nature 410: 387-391

Hasan S, Stucki M, Hassa PO, Imhof R, Gehrig P, Hunziker P, Hubscher U, Hottiger MO (2001b) Regulation of human flap endonuclease-1 activity by acetylation through the transcriptional coactivator p300. Mol Cell 7: 1221-1231

Haukanes BI, Doetsch PW, Olsen LC, Huq I, Krokan HE, Helland DE (1990) Damage specific mammalian endonucleases. Basic Life Sci 53: 191-202

Hazra TK, Hill JW, Izumi T, Mitra S (2001) Multiple DNA glycosylases for repair of 8-oxoguanine and their potential in vivo functions. Prog Nucleic Acid Res Mol Biol 68: 193-205

He Z, Henricksen LA, Wold MS, Ingles CJ (1995) RPA involvement in the damage-recognition and incision steps of nucleotide excision repair. Nature 374: 566-569

Hearst JE, Isaacs ST, Kanne D, Rapoport H, Straub K (1984) The reaction of the psoralens with deoxyribonucleic acid. $Q$ Rev Biophys 17: 1-44

Hendel A, Krijger PH, Diamant N, Goren Z, Langerak P, Kim J, Reissner T, Lee KY, Geacintov NE, Carell T, Myung K, Tateishi S, D'Andrea A, Jacobs H, Livneh Z (2011) PCNA ubiquitination is important, but not essential for translesion DNA synthesis in mammalian cells. PLoS Genet 7: e1002262

Henneke G, Koundrioukoff S, Hubscher U (2003) Phosphorylation of human Fen1 by cyclindependent kinase modulates its role in replication fork regulation. Oncogene 22: 4301-4313

Henning KA, Li L, Iyer N, McDaniel LD, Reagan MS, Legerski R, Schultz RA, Stefanini M, Lehmann AR, Mayne LV, Friedberg EC (1995) The Cockayne syndrome group A gene encodes a WD repeat protein that interacts with CSB protein and a subunit of RNA polymerase II TFIIH. Cell 82: 555-564

Hess MT, Gunz D, Luneva N, Geacintov NE, Naegeli H (1997a) Base pair conformation-dependent excision of benzo[a]pyrene diol epoxide-guanine adducts by human nucleotide excision repair enzymes. Mol Cell Biol 17: 7069-7076

Hess MT, Schwitter U, Petretta M, Giese B, Naegeli H (1997b) Bipartite substrate discrimination by human nucleotide excision repair. Proceedings of the National Academy of Sciences of the United States of America 94: 6664-6669 
Hibbert RG, Sixma TK (2012) Intrinsic flexibility of ubiquitin on proliferating cell nuclear antigen (PCNA) in translesion synthesis. J Biol Chem 287: 39216-39223

Hicks JK, Chute CL, Paulsen MT, Ragland RL, Howlett NG, Gueranger Q, Glover TW, Canman CE (2010) Differential roles for DNA polymerases eta, zeta, and REV1 in lesion bypass of intrastrand versus interstrand DNA cross-links. Mol Cell Biol 30: 1217-1230

Hirai Y, Kodama Y, Moriwaki S, Noda A, Cullings HM, Macphee DG, Kodama K, Mabuchi K, Kraemer $\mathrm{KH}$, Land CE, Nakamura N (2006) Heterozygous individuals bearing a founder mutation in the XPA DNA repair gene comprise nearly $1 \%$ of the Japanese population. Mutation research 601: 171-178

Hishiki A, Hashimoto H, Hanafusa T, Kamei K, Ohashi E, Shimizu T, Ohmori H, Sato M (2009) Structural basis for novel interactions between human translesion synthesis polymerases and proliferating cell nuclear antigen. J Biol Chem 284: 10552-10560

Hoege C, Pfander B, Moldovan GL, Pyrowolakis G, Jentsch S (2002) RAD6-dependent DNA repair is linked to modification of PCNA by ubiquitin and SUMO. Nature 419: 135-141

Hoeijmakers JH (2001) Genome maintenance mechanisms for preventing cancer. Nature 411: 366374

Hoeijmakers JH (2009) DNA damage, aging, and cancer. N Engl J Med 361: 1475-1485

Hofmann K (2009) Ubiquitin-binding domains and their role in the DNA damage response. DNA Repair (Amst) 8: 544-556

Hohl M, Dunand-Sauthier I, Staresincic L, Jaquier-Gubler P, Thorel F, Modesti M, Clarkson SG, Scharer OD (2007) Domain swapping between FEN-1 and XPG defines regions in XPG that mediate nucleotide excision repair activity and substrate specificity. Nucleic acids research 35: 3053-3063

Hohl M, Thorel F, Clarkson SG, Scharer OD (2003) Structural determinants for substrate binding and catalysis by the structure-specific endonuclease XPG. J Biol Chem 278: 19500-19508

Hou C, Zhang T, Tian L, Huang J, Gu L, Li GM (2011) The Role of XPG in Processing (CAG)n/(CTG)n DNA Hairpins. Cell Biosci 1: 11

Houle JF, Friedberg EC (1999) The Drosophila ortholog of the human XPG gene. Gene 234: 353-360

Hu J, Choi JH, Gaddameedhi S, Kemp MG, Reardon JT, Sancar A (2013) Nucleotide excision repair in human cells: fate of the excised oligonucleotide carrying DNA damage in vivo. J Biol Chem 288: 20918-20926 
Hu W, Pan J, Zhao P, Yang G, Yang S (2014) Genetic polymorphisms in XPG could predict clinical outcome of platinum-based chemotherapy for advanced non-small cell lung cancer. Tumour Biol

Hyun M, Park S, Kim E, Kim DH, Lee SJ, Koo HS, Seo YS, Ahn B (2012) Physical and functional interactions of Caenorhabditis elegans WRN-1 helicase with RPA-1. Biochemistry 51: 1336-1345

Ikehata H, Ono T (2011) The mechanisms of UV mutagenesis. J Radiat Res 52: 115-125

Indiani C, Mclnerney P, Georgescu R, Goodman MF, O'Donnell M (2005) A sliding-clamp toolbelt binds high- and low-fidelity DNA polymerases simultaneously. Mol Cell 19: 805-815

Ito S, Kuraoka I, Chymkowitch P, Compe E, Takedachi A, Ishigami C, Coin F, Egly JM, Tanaka K (2007) XPG stabilizes TFIIH, allowing transactivation of nuclear receptors: implications for Cockayne syndrome in XP-G/CS patients. Mol Cell 26: 231-243

Itoh T, Mori T, Ohkubo H, Yamaizumi M (1999) A newly identified patient with clinical xeroderma pigmentosum phenotype has a non-sense mutation in the DDB2 gene and incomplete repair in (6-4) photoproducts. J Invest Dermatol 113: 251-257

lyer N, Reagan MS, Wu KJ, Canagarajah B, Friedberg EC (1996) Interactions involving the human RNA polymerase II transcription/nucleotide excision repair complex TFIIH, the nucleotide excision repair protein XPG, and Cockayne syndrome group B (CSB) protein. Biochemistry 35: 2157-2167

Jackson SP, Bartek J (2009) The DNA-damage response in human biology and disease. Nature 461: 1071-1078

Jazayeri A, Balestrini A, Garner E, Haber JE, Costanzo V (2008) Mre11-Rad50-Nbs1-dependent processing of DNA breaks generates oligonucleotides that stimulate ATM activity. EMBO J 27: 19531962

Jena NR (2012) DNA damage by reactive species: Mechanisms, mutation and repair. J Biosci 37: 503517

Jiang Y, Wang X, Bao S, Guo R, Johnson DG, Shen X, Li L (2010) INO80 chromatin remodeling complex promotes the removal of UV lesions by the nucleotide excision repair pathway. Proceedings of the National Academy of Sciences of the United States of America 107: 17274-17279

Johnson A, Wu R, Peetz M, Gygi SP, Moazed D (2013) Heterochromatic gene silencing by activator interference and a transcription elongation barrier. J Biol Chem 288: 28771-28782

Jonsson ZO, Hubscher U (1997) Proliferating cell nuclear antigen: more than a clamp for DNA polymerases. Bioessays 19: 967-975 
Kamiuchi S, Saijo M, Citterio E, de Jager M, Hoeijmakers JH, Tanaka K (2002) Translocation of Cockayne syndrome group A protein to the nuclear matrix: possible relevance to transcriptioncoupled DNA repair. Proceedings of the National Academy of Sciences of the United States of America 99: 201-206

Kanai S, Kikuno R, Toh H, Ryo H, Todo T (1997) Molecular evolution of the photolyase-blue-light photoreceptor family. J Mol Evol 45: 535-548

Kang MS, Yu SL, Kim HY, Gorospe CM, Choi BH, Lee SH, Lee SK (2014) Yeast RAD2, a homolog of human XPG, plays a key role in the regulation of the cell cycle and actin dynamics. Biol Open 3: 29-41

Kang TH, Reardon JT, Sancar A (2011) Regulation of nucleotide excision repair activity by transcriptional and post-transcriptional control of the XPA protein. Nucleic acids research 39: 31763187

Keijzer W, Jaspers NG, Abrahams PJ, Taylor AM, Arlett CF, Zelle B, Takebe H, Kinmont PD, Bootsma D (1979) A seventh complementation group in excision-deficient xeroderma pigmentosum. Mutation research 62: 183-190

Kelman Z (1997) PCNA: structure, functions and interactions. Oncogene 14: 629-640

Kelman Z, Hurwitz J (1998) Protein-PCNA interactions: a DNA-scanning mechanism? Trends Biochem Sci 23: 236-238

Kemp MG, Reardon JT, Lindsey-Boltz LA, Sancar A (2012) Mechanism of release and fate of excised oligonucleotides during nucleotide excision repair. J Biol Chem 287: 22889-22899

Kim JK, Choi BS (1995) The solution structure of DNA duplex-decamer containing the (6-4) photoproduct of thymidylyl(3'-->5')thymidine by NMR and relaxation matrix refinement. Eur J Biochem 228: 849-854

Kisker C, Kuper J, Van Houten B (2013) Prokaryotic nucleotide excision repair. Cold Spring Harb Perspect Biol 5: a012591

Kleijer WJ, Laugel V, Berneburg M, Nardo T, Fawcett H, Gratchev A, Jaspers NG, Sarasin A, Stefanini $M$, Lehmann AR (2008) Incidence of DNA repair deficiency disorders in western Europe: Xeroderma pigmentosum, Cockayne syndrome and trichothiodystrophy. DNA Repair (Amst) 7: 744-750

Klungland A, Hoss M, Gunz D, Constantinou A, Clarkson SG, Doetsch PW, Bolton PH, Wood RD, Lindahl T (1999) Base excision repair of oxidative DNA damage activated by XPG protein. Mol Cell 3: 33-42

Klungland A, Lindahl T (1997) Second pathway for completion of human DNA base excision-repair: reconstitution with purified proteins and requirement for DNase IV (FEN1). EMBO J 16: 3341-3348 
Knauf JA, Pendergrass SH, Marrone BL, Strniste GF, Maclnnes MA, Park MS (1996) Multiple nuclear localization signals in XPG nuclease. Mutation research 363: 67-75

Kraemer KH (1997) Sunlight and skin cancer: another link revealed. Proceedings of the National Academy of Sciences of the United States of America 94: 11-14

Kraemer KH, Coon HG, Petinga RA, Barrett SF, Rahe AE, Robbins JH (1975a) Genetic heterogeneity in xeroderma pigmentosum: complementation groups and their relationship to DNA repair rates. Proceedings of the National Academy of Sciences of the United States of America 72: 59-63

Kraemer KH, De Weerd-Kastelein EA, Robbins JH, Keijzer W, Barrett SF, Petinga RA, Bootsma D (1975b) Five complementation groups in xeroderma pigmentosum. Mutation research 33: 327-340

Kraemer KH DJ. (2003-2014) Xeroderma Pigmentosum. In Pagon RA AM, Bird TD, et al. (ed.), GeneReviews. Seattle (WA): University of Washington, Seattle; 1993-2014, http://www.ncbi.nlm.nih.gov/books/NBK1397/, Vol. 2003.

Kraemer KH, Lee MM, Scotto J (1987) Xeroderma pigmentosum. Cutaneous, ocular, and neurologic abnormalities in 830 published cases. Arch Dermatol 123: 241-250

Kraemer KH, Patronas NJ, Schiffmann R, Brooks BP, Tamura D, DiGiovanna JJ (2007) Xeroderma pigmentosum, trichothiodystrophy and Cockayne syndrome: a complex genotype-phenotype relationship. Neuroscience 145: 1388-1396

Krasikova YS, Rechkunova NI, Maltseva EA, Petruseva IO, Lavrik OI (2010) Localization of xeroderma pigmentosum group $A$ protein and replication protein A on damaged DNA in nucleotide excision repair. Nucleic acids research 38: 8083-8094

Krishna TS, Fenyo D, Kong XP, Gary S, Chait BT, Burgers P, Kuriyan J (1994a) Crystallization of proliferating cell nuclear antigen (PCNA) from Saccharomyces cerevisiae. Journal of molecular biology 241: $265-268$

Krishna TS, Kong XP, Gary S, Burgers PM, Kuriyan J (1994b) Crystal structure of the eukaryotic DNA polymerase processivity factor PCNA. Cell 79: 1233-1243

Kuper J, Kisker C (2012) Damage recognition in nucleotide excision DNA repair. Curr Opin Struct Biol 22: $88-93$

Kuraoka I, Ito S, Wada T, Hayashida M, Lee L, Saijo M, Nakatsu Y, Matsumoto M, Matsunaga T, Handa $H$, Qin J, Nakatani Y, Tanaka K (2008) Isolation of XAB2 complex involved in pre-mRNA splicing, transcription, and transcription-coupled repair. J Biol Chem 283: 940-950 
Laemmli UK (1970) Cleavage of structural proteins during the assembly of the head of bacteriophage T4. Nature 227: 680-685

Lafrance-Vanasse J, Arseneault G, Cappadocia L, Legault P, Omichinski JG (2013) Structural and functional evidence that Rad4 competes with Rad2 for binding to the Tfb1 subunit of TFIIH in NER. Nucleic acids research 41: 2736-2745

Lagerwerf S, Vrouwe MG, Overmeer RM, Fousteri MI, Mullenders LH (2011) DNA damage response and transcription. DNA Repair (Amst) 10: 743-750

Lalle P, Nouspikel T, Constantinou A, Thorel F, Clarkson SG (2002) The founding members of xeroderma pigmentosum group $G$ produce XPG protein with severely impaired endonuclease activity. J Invest Dermatol 118: 344-351

Larochelle S, Amat R, Glover-Cutter K, Sanso M, Zhang C, Allen JJ, Shokat KM, Bentley DL, Fisher RP (2012) Cyclin-dependent kinase control of the initiation-to-elongation switch of RNA polymerase II. Nat Struct Mol Biol 19: 1108-1115

Le May N, Egly JM, Coin F (2010) True lies: the double life of the nucleotide excision repair factors in transcription and DNA repair. J Nucleic Acids 2010

Le May N, Fradin D, Iltis I, Bougneres P, Egly JM (2012) XPG and XPF endonucleases trigger chromatin looping and DNA demethylation for accurate expression of activated genes. Mol Cell 47: 622-632

Lee BI, Wilson DM, 3rd (1999) The RAD2 domain of human exonuclease 1 exhibits $5^{\prime}$ to $3^{\prime}$ exonuclease and flap structure-specific endonuclease activities. J Biol Chem 274: 37763-37769

Lee SK, Yu SL, Prakash L, Prakash S (2002) Requirement of yeast RAD2, a homolog of human XPG gene, for efficient RNA polymerase II transcription. implications for Cockayne syndrome. Cell 109: 823-834

Lehmann AR (2011) DNA polymerases and repair synthesis in NER in human cells. DNA Repair (Amst) 10: $730-733$

Lehmann AR, Niimi A, Ogi T, Brown S, Sabbioneda S, Wing JF, Kannouche PL, Green CM (2007) Translesion synthesis: Y-family polymerases and the polymerase switch. DNA Repair (Amst) 6: 891899

Lehmann AR, Stevens S (1980) A rapid procedure for measurement of DNA repair in human fibroblasts and for complementation analysis of xeroderma pigmentosum cells. Mutation research 69: $177-190$ 
Lehmann J (2013) Characterization of novel Xeroderma Pigmentosum Group G spontaneous mRNA splicevariants Master Thesis, Department of Dermatology,Venereology and Allergology, GeorgAugust University Göttingen, University medical center (UMG), SUB Göttingen, Germany

Li C, Yin M, Wang LE, Amos Cl, Zhu D, Lee JE, Gershenwald JE, Grimm EA, Wei Q (2013a) Polymorphisms of nucleotide excision repair genes predict melanoma survival. J Invest Dermatol 133: 1813-1821

Li J, Holzschu DL, Sugiyama T (2013b) PCNA is efficiently loaded on the DNA recombination intermediate to modulate polymerase delta, eta, and zeta activities. Proceedings of the National Academy of Sciences of the United States of America 110: 7672-7677

Li JJ, Kelly TJ (1984) Simian virus 40 DNA replication in vitro. Proceedings of the National Academy of Sciences of the United States of America 81: 6973-6977

Li L, Elledge SJ, Peterson CA, Bales ES, Legerski RJ (1994) Specific association between the human DNA repair proteins XPA and ERCC1. Proceedings of the National Academy of Sciences of the United States of America 91: 5012-5016

Li W, Nagaraja S, Delcuve GP, Hendzel MJ, Davie JR (1993) Effects of histone acetylation, ubiquitination and variants on nucleosome stability. Biochem J 296 ( Pt 3): 737-744

Li X, Li J, Harrington J, Lieber MR, Burgers PM (1995) Lagging strand DNA synthesis at the eukaryotic replication fork involves binding and stimulation of FEN-1 by proliferating cell nuclear antigen. J Biol Chem 270: 22109-22112

Li Z, Musich PR, Serrano MA, Dong Z, Zou Y (2011a) XPA-mediated regulation of global nucleotide excision repair by ATR Is p53-dependent and occurs primarily in S-phase. PLoS One 6: e28326

Li Z, Musich PR, Zou Y (2011b) Differential DNA damage responses in p53 proficient and deficient cells: cisplatin-induced nuclear import of XPA is independent of ATR checkpoint in p53-deficient lung cancer cells. Int J Biochem Mol Biol 2: 138-145

Liang C, Kraemer KH, Morris A, Schiffmann R, Price VH, Menefee E, DiGiovanna JJ (2005) Characterization of tiger-tail banding and hair shaft abnormalities in trichothiodystrophy. J Am Acad Dermatol 52: 224-232

Lieber MR (1997) The FEN-1 family of structure-specific nucleases in eukaryotic DNA replication, recombination and repair. Bioessays 19: 233-240

Limsirichaikul S, Niimi A, Fawcett H, Lehmann A, Yamashita S, Ogi T (2009) A rapid non-radioactive technique for measurement of repair synthesis in primary human fibroblasts by incorporation of ethynyl deoxyuridine (EdU). Nucleic acids research 37: e31 
Lindahl T (1993) Instability and decay of the primary structure of DNA. Nature 362: 709-715

Liu R, Qiu J, Finger LD, Zheng L, Shen B (2006) The DNA-protein interaction modes of FEN-1 with gap substrates and their implication in preventing duplication mutations. Nucleic acids research 34: 17721784

Lombard DB, Chua KF, Mostoslavsky R, Franco S, Gostissa M, Alt FW (2005) DNA repair, genome stability, and aging. Cell 120: 497-512

Lyamichev V, Brow MA, Dahlberg JE (1993) Structure-specific endonucleolytic cleavage of nucleic acids by eubacterial DNA polymerases. Science 260: 778-783

Mahindra P, DiGiovanna JJ, Tamura D, Brahim JS, Hornyak TJ, Stern JB, Lee CC, Khan SG, Brooks BP, Smith JA, Driscoll BP, Montemarano AD, Sugarman K, Kraemer KH (2008) Skin cancers, blindness, and anterior tongue mass in African brothers. J Am Acad Dermatol 59: 881-886

Markovitsi D, Gustavsson T, Banyasz A (2010) Absorption of UV radiation by DNA: spatial and temporal features. Mutation research 704: 21-28

Masuda Y, Piao J, Kamiya K (2010) DNA replication-coupled PCNA mono-ubiquitination and polymerase switching in a human in vitro system. Journal of molecular biology 396: 487-500

Masuda $Y$, Suzuki M, Kawai H, Hishiki A, Hashimoto $H$, Masutani $C$, Hishida T, Suzuki F, Kamiya $K$ (2012) En bloc transfer of polyubiquitin chains to PCNA in vitro is mediated by two different human E2-E3 pairs. Nucleic acids research 40: 10394-10407

Masuda Y, Suzuki M, Piao J, Gu Y, Tsurimoto T, Kamiya K (2007) Dynamics of human replication factors in the elongation phase of DNA replication. Nucleic acids research 35: 6904-6916

Masutani C, Kusumoto R, Iwai S, Hanaoka F (2000) Mechanisms of accurate translesion synthesis by human DNA polymerase eta. EMBO J 19: 3100-3109

Mathieu N, Kaczmarek N, Ruthemann P, Luch A, Naegeli H (2013) DNA quality control by a lesion sensor pocket of the xeroderma pigmentosum group D helicase subunit of TFIIH. Curr Biol 23: 204212

Matsumiya S, Ishino S, Ishino Y, Morikawa K (2002) Physical interaction between proliferating cell nuclear antigen and replication factor C from Pyrococcus furiosus. Genes Cells 7: 911-922

Matsumoto Y (2001) Molecular mechanism of PCNA-dependent base excision repair. Prog Nucleic Acid Res Mol Biol 68: 129-138 
McCready SJ, Osman F, Yasui A (2000) Repair of UV damage in the fission yeast Schizosaccharomyces pombe. Mutation research 451: 197-210

McCulloch SD, Kokoska RJ, Masutani C, Iwai S, Hanaoka F, Kunkel TA (2004) Preferential cis-syn thymine dimer bypass by DNA polymerase eta occurs with biased fidelity. Nature 428: 97-100

McHugh PJ, Spanswick VJ, Hartley JA (2001) Repair of DNA interstrand crosslinks: molecular mechanisms and clinical relevance. Lancet Oncol 2: 483-490

Melis JP, van Steeg H, Luijten M (2013) Oxidative DNA damage and nucleotide excision repair. Antioxid Redox Signal 18: 2409-2419

Miyachi K, Fritzler MJ, Tan EM (1978) Autoantibody to a nuclear antigen in proliferating cells. J Immunol 121: 2228-2234

Mocquet V, Laine JP, Riedl T, Yajin Z, Lee MY, Egly JM (2008) Sequential recruitment of the repair factors during NER: the role of XPG in initiating the resynthesis step. EMBO J 27: 155-167

Moggs JG, Yarema KJ, Essigmann JM, Wood RD (1996) Analysis of incision sites produced by human cell extracts and purified proteins during nucleotide excision repair of a 1,3-intrastrand $d(G p T p G)$ cisplatin adduct. J Biol Chem 271: 7177-7186

Montecucco A, Rossi R, Levin DS, Gary R, Park MS, Motycka TA, Ciarrocchi G, Villa A, Biamonti G, Tomkinson AE (1998) DNA ligase I is recruited to sites of DNA replication by an interaction with proliferating cell nuclear antigen: identification of a common targeting mechanism for the assembly of replication factories. EMBO J 17: 3786-3795

Mori T, Nakane M, Hattori T, Matsunaga T, Ihara M, Nikaido O (1991) Simultaneous establishment of monoclonal antibodies specific for either cyclobutane pyrimidine dimer or (6-4)photoproduct from the same mouse immunized with ultraviolet-irradiated DNA. Photochem Photobiol 54: 225-232

Morita R, Nakane S, Shimada A, Inoue M, lino H, Wakamatsu T, Fukui K, Nakagawa N, Masui R, Kuramitsu S (2010) Molecular mechanisms of the whole DNA repair system: a comparison of bacterial and eukaryotic systems. J Nucleic Acids 2010: 179594

Moriwaki S, Stefanini M, Lehmann AR, Hoeijmakers JH, Robbins JH, Rapin I, Botta E, Tanganelli B, Vermeulen W, Broughton BC, Kraemer KH (1996) DNA repair and ultraviolet mutagenesis in cells from a new patient with xeroderma pigmentosum group $G$ and cockayne syndrome resemble xeroderma pigmentosum cells. J Invest Dermatol 107: 647-653

Moser J, Kool H, Giakzidis I, Caldecott K, Mullenders LH, Fousteri MI (2007) Sealing of chromosomal DNA nicks during nucleotide excision repair requires XRCC1 and DNA ligase III alpha in a cell-cyclespecific manner. Mol Cell 27: 311-323 
Mossi R, Hubscher U (1998) Clamping down on clamps and clamp loaders--the eukaryotic replication factor C. Eur J Biochem 254: 209-216

Mu D, Park CH, Matsunaga T, Hsu DS, Reardon JT, Sancar A (1995) Reconstitution of human DNA repair excision nuclease in a highly defined system. J Biol Chem 270: 2415-2418

Mueser TC, Nossal NG, Hyde CC (1996) Structure of bacteriophage T4 RNase H, a 5' to 3' RNA-DNA and DNA-DNA exonuclease with sequence similarity to the RAD2 family of eukaryotic proteins. Cell 85: $1101-1112$

Mullis K, Faloona F, Scharf S, Saiki R, Horn G, Erlich H (1986) Specific enzymatic amplification of DNA in vitro: the polymerase chain reaction. Cold Spring Harbor symposia on quantitative biology 51 Pt 1: 263-273

Naegeli H, Bardwell L, Friedberg EC (1992) The DNA helicase and adenosine triphosphatase activities of yeast Rad3 protein are inhibited by DNA damage. A potential mechanism for damage-specific recognition. J Biol Chem 267: 392-398

Nakatsu Y, Asahina H, Citterio E, Rademakers S, Vermeulen W, Kamiuchi S, Yeo JP, Khaw MC, Saijo M, Kodo N, Matsuda T, Hoeijmakers JH, Tanaka K (2000) XAB2, a novel tetratricopeptide repeat protein involved in transcription-coupled DNA repair and transcription. J Biol Chem 275: 34931-34937

Nance MA, Berry SA (1992) Cockayne syndrome: review of 140 cases. Am J Med Genet 42: 68-84

Newman JC, Bailey AD, Weiner AM (2006) Cockayne syndrome group B protein (CSB) plays a general role in chromatin maintenance and remodeling. Proceedings of the National Academy of Sciences of the United States of America 103: 9613-9618

Nishi R, Sakai W, Tone D, Hanaoka F, Sugasawa K (2013) Structure-function analysis of the EF-hand protein centrin-2 for its intracellular localization and nucleotide excision repair. Nucleic acids research 41: 6917-6929

Nouspikel T (2009) DNA repair in mammalian cells : Nucleotide excision repair: variations on versatility. Cell Mol Life Sci 66: 994-1009

Nouspikel T, Lalle P, Leadon SA, Cooper PK, Clarkson SG (1997) A common mutational pattern in Cockayne syndrome patients from xeroderma pigmentosum group G: implications for a second XPG function. Proceedings of the National Academy of Sciences of the United States of America 94: 31163121

O'Donovan A, Davies AA, Moggs JG, West SC, Wood RD (1994a) XPG endonuclease makes the 3' incision in human DNA nucleotide excision repair. Nature 371: 432-435 
O'Donovan A, Scherly D, Clarkson SG, Wood RD (1994b) Isolation of active recombinant XPG protein, a human DNA repair endonuclease. J Biol Chem 269: 15965-15968

Oberdoerffer P, Sinclair DA (2007) The role of nuclear architecture in genomic instability and ageing. Nat Rev Mol Cell Biol 8: 692-702

Odell ID, Cook D (2013) Immunofluorescence techniques. J Invest Dermatol 133: e4

Ogi T, Limsirichaikul S, Overmeer RM, Volker M, Takenaka K, Cloney R, Nakazawa Y, Niimi A, Miki Y, Jaspers NG, Mullenders LH, Yamashita S, Fousteri MI, Lehmann AR (2010) Three DNA polymerases, recruited by different mechanisms, carry out NER repair synthesis in human cells. Mol Cell 37: 714727

Opperman T, Murli S, Walker GC (1996) The genetic requirements for UmuDC-mediated cold sensitivity are distinct from those for SOS mutagenesis. Journal of bacteriology 178: 4400-4411

Overmeer RM, Gourdin AM, Giglia-Mari A, Kool H, Houtsmuller AB, Siegal G, Fousteri MI, Mullenders LH, Vermeulen W (2010) Replication factor C recruits DNA polymerase delta to sites of nucleotide excision repair but is not required for PCNA recruitment. Mol Cell Biol 30: 4828-4839

Overmeer RM, Moser J, Volker M, Kool H, Tomkinson AE, van Zeeland AA, Mullenders LH, Fousteri M (2011) Replication protein A safeguards genome integrity by controlling NER incision events. J Cell Biol 192: 401-415

Pages V, Fuchs RP (2002) How DNA lesions are turned into mutations within cells? Oncogene 21: 8957-8966

Painter RB, Cleaver JE (1969) Repair replication, unscheduled DNA synthesis, and the repair of mammalian DNA. Radiat Res 37: 451-466

Park CH, Mu D, Reardon JT, Sancar A (1995) The general transcription-repair factor TFIIH is recruited to the excision repair complex by the XPA protein independent of the TFIIE transcription factor. J Biol Chem 270: 4896-4902

Park MS, Knauf JA, Pendergrass SH, Coulon CH, Strniste GF, Marrone BL, Maclnnes MA (1996) Ultraviolet-induced movement of the human DNA repair protein, Xeroderma pigmentosum type $G$, in the nucleus. Proceedings of the National Academy of Sciences of the United States of America 93: 8368-8373

Patel N, Atack JM, Finger LD, Exell JC, Thompson P, Tsutakawa S, Tainer JA, Williams DM, Grasby JA (2012) Flap endonucleases pass 5'-flaps through a flexible arch using a disorder-thread-order mechanism to confer specificity for free 5'-ends. Nucleic acids research 40: 4507-4519 
Patoli AA, Winter JA, Bunting KA (2013) The UmuC subunit of the E. coli DNA polymerase $V$ shows a unique interaction with the beta-clamp processivity factor. BMC Struct Biol 13: 12

Pettijohn D, Hanawalt P (1964) Evidence for Repair-Replication of Ultraviolet Damaged DNA in Bacteria. Journal of molecular biology 9: 395-410

Pike JE, Burgers PM, Campbell JL, Bambara RA (2009) Pif1 helicase lengthens some Okazaki fragment flaps necessitating Dna2 nuclease/helicase action in the two-nuclease processing pathway. J Biol Chem 284: 25170-25180

Pike JE, Henry RA, Burgers PM, Campbell JL, Bambara RA (2010) An alternative pathway for Okazaki fragment processing: resolution of fold-back flaps by Pif1 helicase. J Biol Chem 285: 41712-41723

Podust VN, Podust LM, Goubin F, Ducommun B, Hubscher U (1995) Mechanism of inhibition of proliferating cell nuclear antigen-dependent DNA synthesis by the cyclin-dependent kinase inhibitor p21. Biochemistry 34: 8869-8875

Prakash AS, Tran HP, Peng C, Koyalamudi SR, Dameron CT (2000) Kinetics of DNA alkylation, depurination and hydrolysis of anti diol epoxide of benzo(a)pyrene and the effect of cadmium on DNA alkylation. Chem Biol Interact 125: 133-150

Prasad R, Beard WA, Batra VK, Liu Y, Shock DD, Wilson SH (2011) A review of recent experiments on step-to-step "hand-off" of the DNA intermediates in mammalian base excision repair pathways. Mol Biol (Mosk) 45: 586-600

Prasad R, Dianov GL, Bohr VA, Wilson SH (2000) FEN1 stimulation of DNA polymerase beta mediates an excision step in mammalian long patch base excision repair. J Biol Chem 275: 4460-4466

Prelich G, Kostura M, Marshak DR, Mathews MB, Stillman B (1987a) The cell-cycle regulated proliferating cell nuclear antigen is required for SV40 DNA replication in vitro. Nature 326: 471-475

Prelich G, Tan CK, Kostura M, Mathews MB, So AG, Downey KM, Stillman B (1987b) Functional identity of proliferating cell nuclear antigen and a DNA polymerase-delta auxiliary protein. Nature 326: $517-520$

Protic-Sabljic M, Kraemer KH (1985) One pyrimidine dimer inactivates expression of a transfected gene in xeroderma pigmentosum cells. Proceedings of the National Academy of Sciences of the United States of America 82: 6622-6626

Pustovalova Y, Maciejewski MW, Korzhnev DM (2013) NMR mapping of PCNA interaction with translesion synthesis DNA polymerase Rev1 mediated by Rev1-BRCT domain. Journal of molecular biology 425: 3091-3105 
Qin Z, Lu M, Xu X, Hanna M, Shiomi N, Xiao W (2013) DNA-damage tolerance mediated by PCNA*Ub fusions in human cells is dependent on Rev1 but not Poleta. Nucleic acids research 41: 7356-7369

Qiu J, Guan MX, Bailis AM, Shen B (1998) Saccharomyces cerevisiae exonuclease-1 plays a role in UV resistance that is distinct from nucleotide excision repair. Nucleic acids research 26: 3077-3083

Qiu J, Li X, Frank G, Shen B (2001) Cell cycle-dependent and DNA damage-inducible nuclear localization of FEN-1 nuclease is consistent with its dual functions in DNA replication and repair. J Biol Chem 276: 4901-4908

Rahmeh AA, Zhou Y, Xie B, Li H, Lee EY, Lee MY (2012) Phosphorylation of the p68 subunit of Pol delta acts as a molecular switch to regulate its interaction with PCNA. Biochemistry 51: 416-424

Rasmussen RE, Painter RB (1964) Evidence for Repair of Ultra-Violet Damaged Deoxyribonucleic Acid in Cultured Mammalian Cells. Nature 203: 1360-1362

Rastogi RP, Richa, Kumar A, Tyagi MB, Sinha RP (2010) Molecular mechanisms of ultraviolet radiation-induced DNA damage and repair. J Nucleic Acids 2010: 592980

Ray A, Milum K, Battu A, Wani G, Wani AA (2013) NER initiation factors, DDB2 and XPC, regulate UV radiation response by recruiting ATR and ATM kinases to DNA damage sites. DNA Repair (Amst) 12: 273-283

Reardon JT, Mu D, Sancar A (1996) Overproduction, purification, and characterization of the XPC subunit of the human DNA repair excision nuclease. J Biol Chem 271: 19451-19456

Riedl T, Hanaoka F, Egly JM (2003) The comings and goings of nucleotide excision repair factors on damaged DNA. EMBO J 22: 5293-5303

Rossi ML, Bambara RA (2006) Reconstituted Okazaki fragment processing indicates two pathways of primer removal. J Biol Chem 281: 26051-26061

Rossi ML, Ghosh AK, Bohr VA (2010) Roles of Werner syndrome protein in protection of genome integrity. DNA Repair (Amst) 9: 331-344

Rossi R, Villa A, Negri C, Scovassi I, Ciarrocchi G, Biamonti G, Montecucco A (1999) The replication factory targeting sequence/PCNA-binding site is required in $G(1)$ to control the phosphorylation status of DNA ligase I. EMBO J 18: 5745-5754

Rouillon C, White MF (2011) The evolution and mechanisms of nucleotide excision repair proteins. Res Microbiol 162: 19-26 
Rubbi CP, Milner J (2003) Disruption of the nucleolus mediates stabilization of p53 in response to DNA damage and other stresses. EMBO J 22: 6068-6077

Ryu GH, Tanaka H, Kim DH, Kim JH, Bae SH, Kwon YN, Rhee JS, MacNeill SA, Seo YS (2004) Genetic and biochemical analyses of Pfh1 DNA helicase function in fission yeast. Nucleic acids research 32: 4205-4216

Saharia A, Guittat L, Crocker S, Lim A, Steffen M, Kulkarni S, Stewart SA (2008) Flap endonuclease 1 contributes to telomere stability. Curr Biol 18: 496-500

Saijo M, Takedachi A, Tanaka K (2011) Nucleotide excision repair by mutant xeroderma pigmentosum group A (XPA) proteins with deficiency in interaction with RPA. J Biol Chem 286: 5476-5483

Sakamoto S, Nishikawa K, Heo SJ, Goto M, Furuichi Y, Shimamoto A (2001) Werner helicase relocates into nuclear foci in response to DNA damaging agents and co-localizes with RPA and Rad51. Genes Cells 6: 421-430

Sale JE, Lehmann AR, Woodgate R (2012) Y-family DNA polymerases and their role in tolerance of cellular DNA damage. Nat Rev Mol Cell Biol 13: 141-152

Sanger F, Nicklen S, Coulson AR (1977) DNA sequencing with chain-terminating inhibitors. Proceedings of the National Academy of Sciences of the United States of America 74: 5463-5467

Sarker AH, Tsutakawa SE, Kostek S, Ng C, Shin DS, Peris M, Campeau E, Tainer JA, Nogales E, Cooper PK (2005) Recognition of RNA polymerase II and transcription bubbles by XPG, CSB, and TFIIH: insights for transcription-coupled repair and Cockayne Syndrome. Mol Cell 20: 187-198

Sattler U, Frit P, Salles B, Calsou P (2003) Long-patch DNA repair synthesis during base excision repair in mammalian cells. EMBO Rep 4: 363-367

Savas S, Ozcelik H (2005) Phosphorylation states of cell cycle and DNA repair proteins can be altered by the nsSNPs. BMC Cancer 5: 107

Schaeffer L, Roy R, Humbert S, Moncollin V, Vermeulen W, Hoeijmakers JH, Chambon P, Egly JM (1993) DNA repair helicase: a component of BTF2 (TFIIH) basic transcription factor. Science 260: 5863

Schafer A, Hofmann L, Gratchev A, Laspe P, Schubert S, Schurer A, Ohlenbusch A, Tzvetkov M, Hallermann C, Reichrath J, Schon MP, Emmert S (2013a) Molecular genetic analysis of 16 XP-C patients from Germany: environmental factors predominately contribute to phenotype variations. Exp Dermatol 22: 24-29

Schafer A, Schubert S, Gratchev A, Seebode C, Apel A, Laspe P, Hofmann L, Ohlenbusch A, Mori T, Kobayashi N, Schurer A, Schon MP, Emmert S (2013b) Characterization of three XPG-defective 
patients identifies three missense mutations that impair repair and transcription. $J$ Invest Dermatol 133: $1841-1849$

Scharer OD (2008a) A molecular basis for damage recognition in eukaryotic nucleotide excision repair. Chembiochem 9: 21-23

Scharer OD (2008b) XPG: its products and biological roles. Adv Exp Med Biol 637: 83-92

Scharer OD (2013) Nucleotide excision repair in eukaryotes. Cold Spring Harb Perspect Biol 5: a012609

Scherer WF, Syverton JT, Gey GO (1953) Studies on the propagation in vitro of poliomyelitis viruses. IV. Viral multiplication in a stable strain of human malignant epithelial cells (strain HeLa) derived from an epidermoid carcinoma of the cervix. J Exp Med 97: 695-710

Scherly D, Nouspikel T, Corlet J, Ucla C, Bairoch A, Clarkson SG (1993) Complementation of the DNA repair defect in xeroderma pigmentosum group $G$ cells by a human CDNA related to yeast RAD2. Nature 363: 182-185

Schubert S, Lehmann J, Kalfon L, Slor H, Falik-Zaccai TC, Emmert S (2013) Clinical utility gene card for: Xeroderma pigmentosum. Eur J Hum Genet

Scrima A, Konickova R, Czyzewski BK, Kawasaki Y, Jeffrey PD, Groisman R, Nakatani Y, Iwai S, Pavletich NP, Thoma NH (2008) Structural basis of UV DNA-damage recognition by the DDB1-DDB2 complex. Cell 135: 1213-1223

Sertic S, Pizzi S, Cloney R, Lehmann AR, Marini F, Plevani P, Muzi-Falconi M (2011) Human exonuclease 1 connects nucleotide excision repair (NER) processing with checkpoint activation in response to UV irradiation. Proceedings of the National Academy of Sciences of the United States of America 108: 13647-13652

Sertic S, Pizzi S, Lazzaro F, Plevani P, Muzi-Falconi M (2012) NER and DDR: classical music with new instruments. Cell Cycle 11: 668-674

Shell SM, Hawkins EK, Tsai MS, Hlaing AS, Rizzo CJ, Chazin WJ (2013) Xeroderma pigmentosum complementation group $C$ protein (XPC) serves as a general sensor of damaged DNA. DNA Repair (Amst) 12: 947-953

Shivji MK, Ferrari E, Ball K, Hubscher U, Wood RD (1998) Resistance of human nucleotide excision repair synthesis in vitro to p21Cdn1. Oncogene 17: 2827-2838

Shivji MK, Moggs JG, Kuraoka I, Wood RD (1999) Dual-incision assays for nucleotide excision repair using DNA with a lesion at a specific site. Methods Mol Biol 113: 373-392 
Sobhy MA, Joudeh LI, Huang X, Takahashi M, Hamdan SM (2013) Sequential and multistep substrate interrogation provides the scaffold for specificity in human flap endonuclease 1. Cell Rep 3: 17851794

Soehnge H, Ouhtit A, Ananthaswamy ON (1997) Mechanisms of induction of skin cancer by UV radiation. Front Biosci 2: d538-551

Soltys DT, Rocha CR, Lerner LK, de Souza TA, Munford V, Cabral F, Nardo T, Stefanini M, Sarasin A, Cabral-Neto JB, Menck CF (2013) Novel XPG (ERCC5) mutations affect DNA repair and cell survival after ultraviolet but not oxidative stress. Hum Mutat 34: 481-489

Soria G, Podhajcer O, Prives C, Gottifredi V (2006) P21Cip1/WAF1 downregulation is required for efficient PCNA ubiquitination after UV irradiation. Oncogene 25: 2829-2838

Staresincic L, Fagbemi AF, Enzlin JH, Gourdin AM, Wijgers N, Dunand-Sauthier I, Giglia-Mari G, Clarkson SG, Vermeulen W, Scharer OD (2009) Coordination of dual incision and repair synthesis in human nucleotide excision repair. EMBO J 28: 1111-1120

Stelter P, Ulrich HD (2003) Control of spontaneous and damage-induced mutagenesis by SUMO and ubiquitin conjugation. Nature 425: 188-191

Storici F, Henneke G, Ferrari E, Gordenin DA, Hubscher U, Resnick MA (2002) The flexible loop of human FEN1 endonuclease is required for flap cleavage during DNA replication and repair. EMBO $J$ 21: $5930-5942$

Sugasawa K, Masutani C, Uchida A, Maekawa T, van der Spek PJ, Bootsma D, Hoeijmakers JH, Hanaoka F (1996) HHR23B, a human Rad23 homolog, stimulates XPC protein in nucleotide excision repair in vitro. Mol Cell Biol 16: 4852-4861

Sugasawa K, Okuda Y, Saijo M, Nishi R, Matsuda N, Chu G, Mori T, Iwai S, Tanaka K, Hanaoka F (2005) UV-induced ubiquitylation of XPC protein mediated by UV-DDB-ubiquitin ligase complex. Cell 121: $387-400$

Suquet C, Smerdon MJ (1993) UV damage to DNA strongly influences its rotational setting on the histone surface of reconstituted nucleosomes. J Biol Chem 268: 23755-23757

Sutton MD, Smith BT, Godoy VG, Walker GC (2000) The SOS response: recent insights into umuDCdependent mutagenesis and DNA damage tolerance. Annu Rev Genet 34: 479-497 
Takahashi E, Shiomi N, Shiomi T (1992) Precise localization of the excision repair gene, ERCC5, to human chromosome 13q32.3-q33.1 by direct R-banding fluorescence in situ hybridization. Jpn J Cancer Res 83: 1117-1119

Takedachi A, Saijo M, Tanaka K (2010) DDB2 complex-mediated ubiquitylation around DNA damage is oppositely regulated by XPC and $\mathrm{Ku}$ and contributes to the recruitment of XPA. Mol Cell Biol 30: 2708-2723

Tang J, Chu G (2002) Xeroderma pigmentosum complementation group E and UV-damaged DNAbinding protein. DNA Repair (Amst) 1: 601-616

Tantin D, Kansal A, Carey M (1997) Recruitment of the putative transcription-repair coupling factor CSB/ERCC6 to RNA polymerase II elongation complexes. Mol Cell Biol 17: 6803-6814

Tapias A, Auriol J, Forget D, Enzlin JH, Scharer OD, Coin F, Coulombe B, Egly JM (2004) Ordered conformational changes in damaged DNA induced by nucleotide excision repair factors. $J$ Biol Chem 279: $19074-19083$

Temviriyanukul P, van Hees-Stuivenberg S, Delbos F, Jacobs H, de Wind N, Jansen JG (2012) Temporally distinct translesion synthesis pathways for ultraviolet light-induced photoproducts in the mammalian genome. DNA Repair (Amst) 11: 550-558

Thorel F, Constantinou A, Dunand-Sauthier I, Nouspikel T, Lalle P, Raams A, Jaspers NG, Vermeulen W, Shivji MK, Wood RD, Clarkson SG (2004) Definition of a short region of XPG necessary for TFIIH interaction and stable recruitment to sites of UV damage. Mol Cell Biol 24: 10670-10680

Tian M, Jones DA, Smith M, Shinkura R, Alt FW (2004) Deficiency in the nuclease activity of xeroderma pigmentosum $\mathrm{G}$ in mice leads to hypersensitivity to UV irradiation. Mol Cell Biol 24: 22372242

Tice RRS, R. B. (1985) DNA repair and replication in aging organisms and cells, Van Nostrand Reinhold, New York.

Tillhon M, Cazzalini O, Nardo T, Necchi D, Sommatis S, Stivala LA, Scovassi Al, Prosperi E (2012) p300/CBP acetyl transferases interact with and acetylate the nucleotide excision repair factor XPG. DNA Repair (Amst) 11: 844-852

Tommasi S, Swiderski PM, Tu Y, Kaplan BE, Pfeifer GP (1996) Inhibition of transcription factor binding by ultraviolet-induced pyrimidine dimers. Biochemistry 35: 15693-15703

Trego KS, Chernikova SB, Davalos AR, Perry JJ, Finger LD, Ng C, Tsai MS, Yannone SM, Tainer JA, Campisi J, Cooper PK (2011) The DNA repair endonuclease XPG interacts directly and functionally with the WRN helicase defective in Werner syndrome. Cell Cycle 10: 1998-2007 
Truglio JJ, Croteau DL, Van Houten B, Kisker C (2006) Prokaryotic nucleotide excision repair: the UvrABC system. Chem Rev 106: 233-252

Tsutakawa SE, Classen S, Chapados BR, Arvai AS, Finger LD, Guenther G, Tomlinson CG, Thompson P, Sarker AH, Shen B, Cooper PK, Grasby JA, Tainer JA (2011) Human flap endonuclease structures, DNA double-base flipping, and a unified understanding of the FEN1 superfamily. Cell 145: 198-211

Tsutakawa SE, Tainer JA (2012) Double strand binding-single strand incision mechanism for human flap endonuclease: implications for the superfamily. Mech Ageing Dev 133: 195-202

Uhlmann F, Gibbs E, Cai J, O'Donnell M, Hurwitz J (1997) Identification of regions within the four small subunits of human replication factor $\mathrm{C}$ required for complex formation and DNA replication. $J$ Biol Chem 272: 10065-10071

Ulrich HD, Takahashi T (2013) Readers of PCNA modifications. Chromosoma 122: 259-274

Ura K, Araki M, Saeki H, Masutani C, Ito T, Iwai S, Mizukoshi T, Kaneda Y, Hanaoka F (2001) ATPdependent chromatin remodeling facilitates nucleotide excision repair of UV-induced DNA lesions in synthetic dinucleosomes. EMBO J 20: 2004-2014

van Steeg H, Kraemer KH (1999) Xeroderma pigmentosum and the role of UV-induced DNA damage in skin cancer. Mol Med Today 5: 86-94

Vignard J, Mirey G, Salles B (2013) lonizing-radiation induced DNA double-strand breaks: A direct and indirect lighting up. Radiother Oncol 108: 362-369

Wakasugi M, Reardon JT, Sancar A (1997) The non-catalytic function of XPG protein during dual incision in human nucleotide excision repair. J Biol Chem 272: 16030-16034

Wakasugi M, Sancar A (1998) Assembly, subunit composition, and footprint of human DNA repair excision nuclease. Proceedings of the National Academy of Sciences of the United States of America 95: 6669-6674

Wang $\mathrm{H}$, Zhai L, Xu J, Joo HY, Jackson S, Erdjument-Bromage $H$, Tempst $P$, Xiong $Y$, Zhang $Y$ (2006) Histone $\mathrm{H} 3$ and $\mathrm{H} 4$ ubiquitylation by the CUL4-DDB-ROC1 ubiquitin ligase facilitates cellular response to DNA damage. Mol Cell 22: 383-394

Warbrick E (2000) The puzzle of PCNA's many partners. Bioessays 22: 997-1006

Warbrick E, Lane DP, Glover DM, Cox LS (1995) A small peptide inhibitor of DNA replication defines the site of interaction between the cyclin-dependent kinase inhibitor p21WAF1 and proliferating cell nuclear antigen. Curr Biol 5: 275-282 
Warbrick E, Lane DP, Glover DM, Cox LS (1997) Homologous regions of Fen1 and p21Cip1 compete for binding to the same site on PCNA: a potential mechanism to co-ordinate DNA replication and repair. Oncogene 14: 2313-2321

Weinfeld M, Gentner NE, Johnson LD, Paterson MC (1986) Photoreversal-dependent release of thymidine and thymidine monophosphate from pyrimidine dimer-containing DNA excision fragments isolated from ultraviolet-damaged human fibroblasts. Biochemistry 25: 2656-2664

Winkler GS, Araujo SJ, Fiedler U, Vermeulen W, Coin F, Egly JM, Hoeijmakers JH, Wood RD, Timmers HT, Weeda G (2000) TFIIH with inactive XPD helicase functions in transcription initiation but is defective in DNA repair. J Biol Chem 275: 4258-4266

Winkler GS, Sugasawa K, Eker AP, de Laat WL, Hoeijmakers JH (2001) Novel functional interactions between nucleotide excision DNA repair proteins influencing the enzymatic activities of TFIIH, XPG, and ERCC1-XPF. Biochemistry 40: 160-165

Wold MS (1997) Replication protein A: a heterotrimeric, single-stranded DNA-binding protein required for eukaryotic DNA metabolism. Annu Rev Biochem 66: 61-92

Wu X, Li J, Li X, Hsieh CL, Burgers PM, Lieber MR (1996) Processing of branched DNA intermediates by a complex of human FEN-1 and PCNA. Nucleic acids research 24: 2036-2043

Yang W, Woodgate R (2007) What a difference a decade makes: insights into translesion DNA synthesis. Proceedings of the National Academy of Sciences of the United States of America 104: 15591-15598

Yao J, Dixon K, Carty MP (2001) A single (6-4) photoproduct inhibits plasmid DNA replication in xeroderma pigmentosum variant cell extracts. Environ Mol Mutagen 38: 19-29

Yeh JI, Levine AS, Du S, Chinte U, Ghodke H, Wang H, Shi H, Hsieh CL, Conway JF, Van Houten B, Rapic-Otrin V (2012) Damaged DNA induced UV-damaged DNA-binding protein (UV-DDB) dimerization and its roles in chromatinized DNA repair. Proceedings of the National Academy of Sciences of the United States of America 109: E2737-2746

Yielding LW, Brown BR, Graves DE, Yielding KL (1979) Ethidium bromide enhancement of frameshift mutagenesis caused by photoactivatable ethidium analogs. Mutation research 63: 225-232

Yokoyama H, Mizutani R, Satow Y, Sato K, Komatsu Y, Ohtsuka E, Nikaido O (2012) Structure of the DNA (6-4) photoproduct dTT(6-4)TT in complex with the 64M-2 antibody Fab fragment implies increased antibody-binding affinity by the flanking nucleotides. Acta Crystallogr D Biol Crystallogr 68: 232-238

Yoon JH, Swiderski PM, Kaplan BE, Takao M, Yasui A, Shen B, Pfeifer GP (1999) Processing of UV damage in vitro by FEN-1 proteins as part of an alternative DNA excision repair pathway. Biochemistry 38: 4809-4817 
Yuli Y, Zhe S, Xia W, Siqing L, Zhenxuan W, Yu-Hua Z, Bing S, Jun-Wei C (2013) XPG is a novel biomarker of clinical outcome in advanced non-small-cell lung cancer. Pak J Med Sci 29: 762-767

Zafeiriou DI, Thorel F, Andreou A, Kleijer WJ, Raams A, Garritsen VH, Gombakis N, Jaspers NG, Clarkson SG (2001) Xeroderma pigmentosum group $G$ with severe neurological involvement and features of Cockayne syndrome in infancy. Pediatr Res 49: 407-412

Zhang F, Scheerer P, Oberpichler I, Lamparter T, Krauss N (2013a) Crystal structure of a prokaryotic (6-4) photolyase with an Fe-S cluster and a 6,7-dimethyl-8-ribityllumazine antenna chromophore. Proceedings of the National Academy of Sciences of the United States of America 110: 7217-7222

Zhang T, Huang J, Gu L, Li GM (2012) In vitro repair of DNA hairpins containing various numbers of CAG/CTG trinucleotide repeats. DNA Repair (Amst) 11: 201-209

Zhang Y, Yuan F, Wu X, Rechkoblit O, Taylor JS, Geacintov NE, Wang Z (2000a) Error-prone lesion bypass by human DNA polymerase eta. Nucleic acids research 28: 4717-4724

Zhang Y, Yuan F, Wu X, Wang M, Rechkoblit O, Taylor JS, Geacintov NE, Wang Z (2000b) Error-free and error-prone lesion bypass by human DNA polymerase kappa in vitro. Nucleic acids research $\mathbf{2 8}$ : 4138-4146

Zhang Z, Pal S, Bi Y, Tchou J, Davuluri RV (2013b) Isoform level expression profiles provide better cancer signatures than gene level expression profiles. Genome Med 5: 33

Zheng L, Jia J, Finger LD, Guo Z, Zer C, Shen B (2011) Functional regulation of FEN1 nuclease and its link to cancer. Nucleic acids research 39: 781-794

Zhu Q, Wani G, Sharma N, Wani A (2012) Lack of CAK complex accumulation at DNA damage sites in XP-B and XP-B/CS fibroblasts reveals differential regulation of CAK anchoring to core TFIIH by XPB and XPD helicases during nucleotide excision repair. DNA Repair (Amst) 11: 942-950

Zorn C, Cremer C, Cremer T, Zimmer J (1979) Unscheduled DNA synthesis after partial UV irradiation of the cell nucleus. Distribution in interphase and metaphase. Exp Cell Res 124: 111-119

Zotter A, Luijsterburg MS, Warmerdam DO, Ibrahim S, Nigg A, van Cappellen WA, Hoeijmakers JH, van Driel R, Vermeulen W, Houtsmuller AB (2006) Recruitment of the nucleotide excision repair endonuclease XPG to sites of UV-induced dna damage depends on functional TFIIH. Mol Cell Biol 26: 8868-8879 


\section{Curriculum vitae}

\section{Steffen Schubert}

Schopenhauerweg 17

37083 Göttingen, GER

Place of birth

Date of birth

Citizenship

Personal status

\section{Education and career}

$04 / 2010$ - 05/2014

04/2009 - 03/2010

05/2008 - 02/2009

10/2002 - 02/2009

09/2001-05/2002

09/1997 - 06/2001

09/1993 - 07/1997

09/1989 - 07/1993 e-mail: sschube4@gwdg.de

phone: 055139 -9655/-6734

Leinefelde, Germany

02/27/1983

German

married

$\mathrm{PhD}$ student (Dermatology, Allergology and Venereology Göttingen, laboratory of Prof. Dr. Steffen Emmert)

Member of a research staff, Georg-August university of Göttingen, Department of Microbiology and Genetics, Department of Molecular Genetics und Preparative Molecular Biology, Prof. Dr. H.-J. Fritz (protein engineering)

Diploma thesis, Georg-August university of Göttingen, Department of Microbiology and Genetics,

Department of Molecular Genetics and Preparative Molecular Biology, Prof. Dr. H.-J. Fritz. "Genetisches Durchmustern einer Proteinbibliothek nach Katalysatoren" (grade 1,85)

Academic studies (Biology) Georg-August University Göttingen; Deepening Direction: Genetics; minor fields of study: business management and anthropology (grade 1,7)

Conscript of Deutsche Bundeswehr; gunner PzArtBtl 2 in Hess. Lichtenau

Student; Albert-Schweitzer-Gymnasium, special section for mathematics and sciences, Erfurt; Abitur (grade: 1,4)

Student; Tilesius-Gymnasium in Mühlhausen

Student; Margareten-Grundschule in Mühlhausen 


\section{Research skills}

Microbiological methods

Molecular methods

Biochemical methods

Cell culture methods

Functional assays

Biological methods

\section{Additional skills}

$08 / 2010$

$09 / 2010$ till now

24./25.11.2009

09./10.12.2009

language

computing
Preparation of highly competent cells (E.coli), transformation techniques

functional complementation, cloning of recombinant DNA; isolation, purification and enzymatic manipulation of DNA/RNA, electrophoresis, PCR, mutagenesis, sequencing, quantitative Real Time-PCR

Expression und purification of recombinant proteins (E.coli), Western blotting, SDS gel electrophoresis, enzyme kinetics

Cell culture, several transfection methods,

HCR (Host-Cell-Reactivation Assay), MTT-Assay (cell survival), ELISA (Enzyme-linked-immuno-(ab)sorbentAssay), UDS (unscheduled DNA-synthesis), immuno fluorescence

Histology, morphological investigations of skeletal bodies and mummies, genetic characterization of individuals (genetic fingerprinting), electron microscopy, X-ray investigations of skeletal bodies

personalized medicine

deputy radiation protection officer Dermatology Göttingen

UNIX course

DNA analysis course

German (mother tongue)

English (fluent written and spoken)

French (fundamentals)

MS Office (Word, PowerPoint, Office)

Vector NTI

Online tools (e.g. Multialign, Blast)

Photoshop 